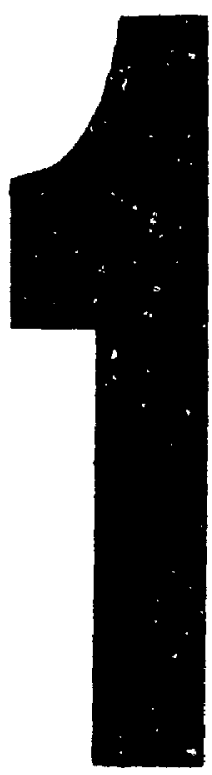

PM-1 31/2" 4 4" PHOTOGRAPHIC MICROCOPY TARGET NBS 1010a ANSI/ISO \#2 EQUIVALENT

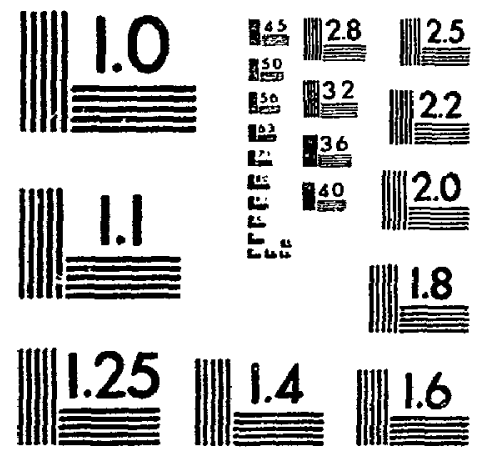

PRECISIONSM RESOLUTION TARGETS 
Bibliographic Services Branch

395 Willington Street

Uttawa. Ontarı

KIA ON.
Direction des acquisitions et

des services bibliographiques

395, rue Wellinglion

Oltar.a (Ontario)

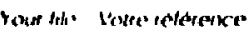

The quality of this microform is heavily dependent upon the quality of the original thesis submitted for microfilming. Every effort has been made to ensure the highest quality of reproduction possible.

If pages are missing, contact the university which granted the degree.

Some pages may have indistinct print especially if the original pages were typed with a poor typewriter ribbon or if the university sent us an inferior photocopy.
La qualité de cette microforme dépend grandement de la qualité de la thèse soumise au microfilmage. Nous avons tout fait pour assurer une qualité supérieure de reproduction.

S'il manque des pages, veuillez communiquer avec l'université qui a conféré ie grade.

La qualité d'impression de certaines pages peut laisser à désirer, surtout si les pages originales ont été dactylographiées à l'aide d'un ruban usé ou si l'université nous a fait parvenir une photocopie de qualité inférieure.

La reproduction, même partielle, de cette microforme est soumise à la Loi canadienne sur le droit d'auteur, SRC 1970, c. C-30, et ses amendements subséquents. 


\title{
The Design and Implementation of CMOS Components for a Gigahertz Frequency Synthesizer
}

\author{
Manop Thamsirianunt, B.Eng.
}

\begin{abstract}
A thesis submitted to the Faculty of Graduate Studies and Research in partial fulfillment of the requirements for the degree of Master of Engineering
\end{abstract}

Department of Electronics, Carleton University

Ottawa, Ontario K1S 5B6

December 1993

(C) Copyright 1993 
National Library

of Canada

Acquisitions and Bibliographic Services Branch

395 Wellington Street

Ottawa, Ontario

K1A ON4
Bibliotheque nationale

du Canada

Direction des acquisitions ef des services bibliographiques

395, tue Wellington

Ottawa (Ontario)

KIAON4

rour the vorro retorence

Our hila Notre retarences

THE AUTHOR HAS GRANTED AN IRREVOCABLE NON-EXCLUSIVE LICENCE ALLOWING THE NATIONAL LIBRARY OF CANADA TO REPRODUCE, LOAN, DISTRIBUTE OR SELL COPIES OF HIS/HER THESIS BY ANY MEANS AND N ANY FORM OR FORMAT, MAKING I HIS THESIS AVAILABLE TO INTERESTED PERSONS.
L'AUTEUR A ACCORDE UNE LICENCE IRREVOCABLE ET NON EXCLUSIVE PERMETTANT A LA BIBLIOTHEQUE NATIONALE DU CANADA DE REPRODUIRE, PRETER, DISTRIBUER OU VENDRE DES COPIES DE SA THESE DE QUELQUE MANIERE ET SOUS QUELQUE FORME QUE CE SOIT POUR METTRE DES EXEMPLAIRES DE CETTE THESE A LA DISPOSITION DES PERSONIE INTERESSEES.
THE AUTHOR RETAINS OWNERSHIP OF THE COPYRIGHT IN HIS/HER THESIS. NEITHER THE THESIS NOR SUBSTANTIAL EXTRACTS FROM IT MAY BE PRINTED OR OTHERWISE REPRODUCED WITHOUT HIS/HER PERMISSION.
L'AUTEUR CONSERVE LA PROPRIETE DU DROIT D'AUTEUR QUI PROTEGE SA THESE. NI LA THESE NI DES EXTRAITS SUBSTANTIELS DE CELLECI NE DOIVENT ETRE IMPRIMES OU AUTREMENT REPRODUITS SANS SON AUTORISATION. 
Name

Dissertation Abstracts International is arranged by broad, general subject categories. Please select the one subject which most nearly describes the content of your dissertation. Enter the corresponding four-digit code in the spaces provided.

\section{Subject Cutegories}

\section{THE HUMaNITIES AND SOCMLL SCIENCES}

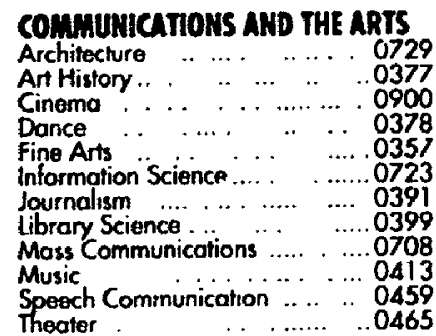

\section{EDUCATION}

Genoral . . . ................0515

Administration $\ldots \ldots . . .0514$

Agricultural 0517

Ant ……

Bilingual and Multicultural ......0282

Business

Community Colliege .................0275

Curriculum and Instruction .....0727

Early Childhood ……........ 0518

Elementary ................0524

Guidance and Cousnseling ..........0519

Heolith ... . ......... 0680

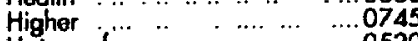

History of ....................0520

Home Economics . ...

Industrial ...... it. .........052

Music

Music ........................... 0522

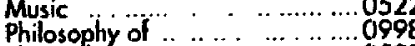

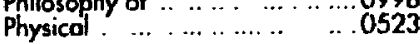

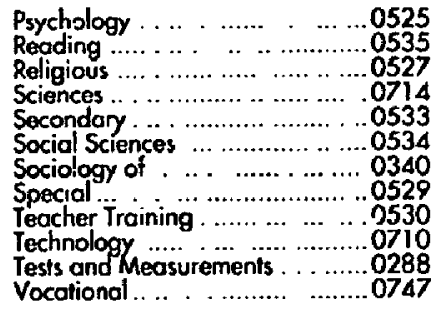

\section{UNGUACE, LITERATURE AND}

LARGUISTICS

Language

General .... ................ 0679

Ancient ......................0289

Linguistics ............... 0290

Literafure

General ................0401

Classical ..................... 0294

Comparative . ................. 0295

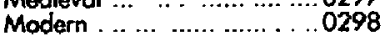

African ............................ 0316

Atrican ..................... 0591

American . .......................0305

Canadian (Ënglish) ...............0352

Canadian (french). ..........0355

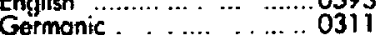

Lot n Americcin ..................... 0312

Middle Eastern ............ . .0315

Romance ............... 0313

Slavic and East European ......0314
PHHOSOPHY, RELGIOH AND

THEOLOGY

Philosophy ............. . . ..........0422

Religion

Generol ...... 0318

Biblical Studies .....................

Clergy ........................0319

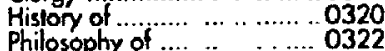

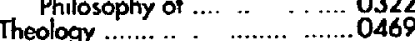

\section{SOCIAL SCIENCES}

American Studies $\quad .0323$

Anthropology

Archoeology ................. 0324

Cultural ............................. 0326

Physical ..........................0327

Business Administration

General ... . . . ............0310

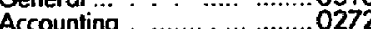

Banking ... .................... 0770

Management ……....... .0454

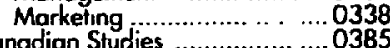

Canadian Studies

conomics

Agricultural .....

Agric llaral ....................0503

Commerce-Business ........0505

Finance ….....................508

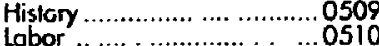

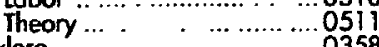

Folklore . . .

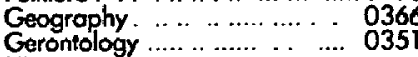

Gerontology

Gen

General

.0578

\section{THE SCIENCES AND ENGHEERING}

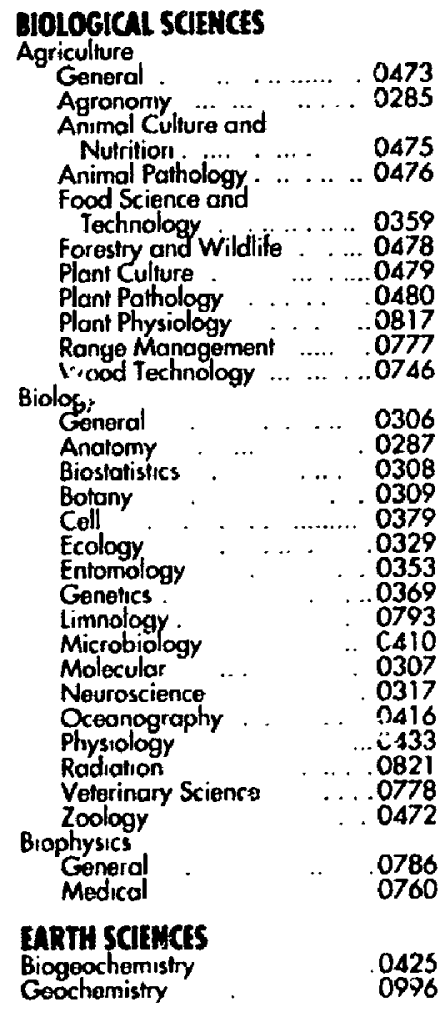

Gzodesy

Geology

Hydrology . ...................... 0388

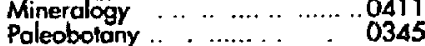

Poleoecology.. ... ...... 0426

Paleontology .................... 0418

Polcozology ......................0985

Polynoiony ................. 0427

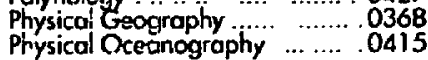

\section{HEALTH AND ENVIRONMENTAL} SCIEHCES

Environmental Sciences $\quad \ldots \quad .0768$ Health Sciences

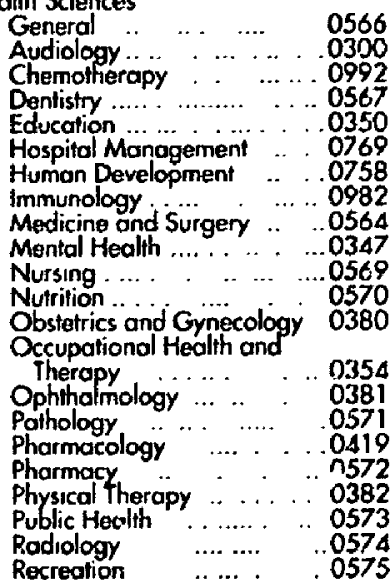

Ancient ... . ......................0579

Modern …....................0582

Black .............................0328

African ............................033

Asa, Australia and Oceanio 0332

Lanadian ...........................0335

Latin American ......................0336

Middle Eastern ....................0333

United States ...................0337

History of Science .....................0585

Low .... .............................0398

General

International Low and

Relations

0616

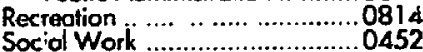

Sosiology

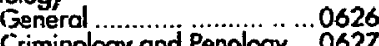

C.riminolngy and Penology ...0627

Eemogruphy .................0631

indiridual and Family

Studies .............

...0628

Industrial and Labor

.0629

Public and Social Welfare ... 0630

Social Structure and

Clevelopment …….......... 0700

Theory and Methods .............0344

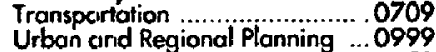

Womein's Studies .................... 0453

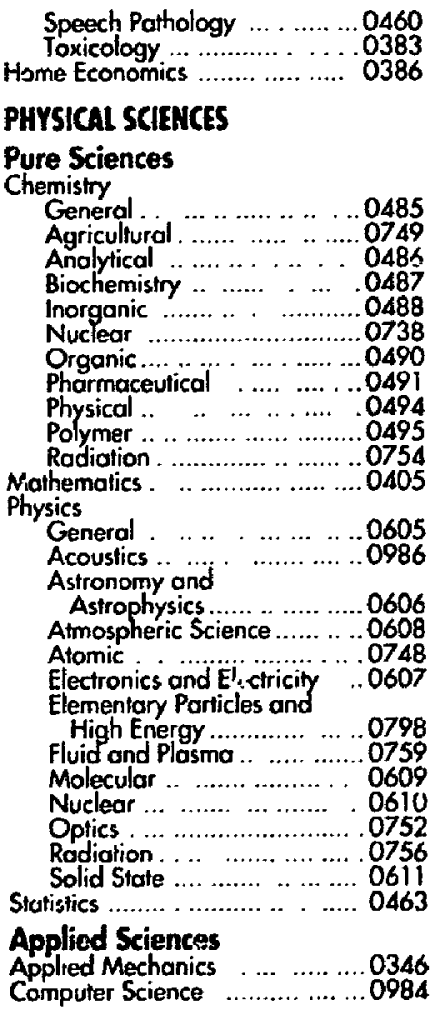

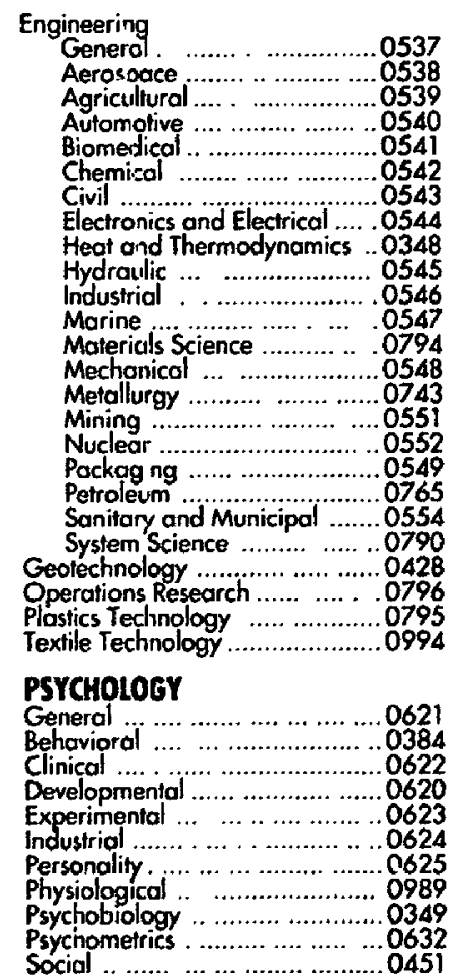

Engineering

.......0539

Autamolive .... 0540

Chemicol .............. 0542

Electronics and Electrical ......054

Heat and Thermodynamics ..0348

Hydranlic ... ............... 0345

Industrial . . .....................0546

Materials Science ............. .0794

Mechonical ... ……….......0548

.........055

Pockg ng

0765

Sanitary and Municipal .......0554

Ceolechnology ….....................042B

Operations Research ........... . 0796

Textile Technology .....................0994

PSYCHOLOGY

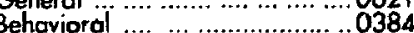

Clinical ..............................0622

clical.....0620

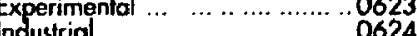

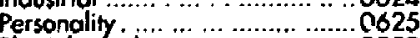

Physiological .. .................... 0989

Pychometrics . .......................65 
The undersigned hereby recommend to the Faculty of Graduate Studies and Research acceptance of the thesis,

\title{
THE DESIGN AND IMPLEMENTATION OF CMOS COMPONENTS FOR A GIGAHERTZ FREQUENCY SYNTHESIZER
}

\author{
submitted by \\ Manop Thamsirianunt, B.Eng.
}

in partial fulfilment of the requirements

for the degree of Master of Engineering.

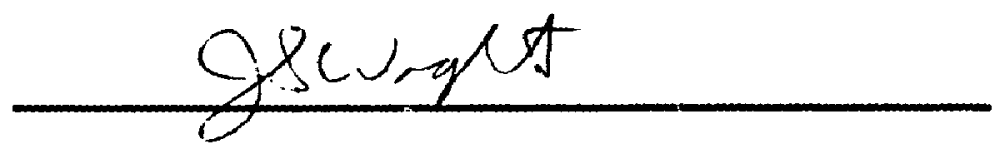

Chair, Department of Electronics

T. Koasnienshlu

Thesis Supervisor

Carleton University

January 1994 


\section{Abstract}

The design and implementation of high-speed CMOS VCO circuits, targeted for use as single-chip frequency synthesizers for digital mobile radio communications systems, is presented. Three types of VCO designs, based on the operation of transistor-onlyparasitic-capacitors have been proposed, with performance and characteristics evaluated through simulation and post-fabrication measurements. The results show that speed and phase noise can be simultaneously optimized. The circuit topology and layout optimization resulted in $\mathbf{G H z}$ operating frequencies and milliwatt range power consumption for both simulation and post-fabrication measurement. An experimental frequency synthesis prototype was built and tested using the frequency synthesizer chip designed in CMOS 1.2- $\mu \mathrm{m}$-gate-length technology. The synthesizer operated at $850 \mathrm{MHz}$ with $-94 \mathrm{dBc} / \mathrm{Hz}$ phase noise at a $1 \mathrm{MHz}$ carrier offset. The work in this thesis demonstrates the feasibility of an all CMOS frequency synthesizer for mobile radio terminal applications. 


\section{Acknowledgements}

First and foremost, I would like to thank my supervisor, Dr. Tadeusz A. Kwasniewski, for his valuable guidance and constant support of this thesis work.

I would also like to thank Terry Kenny who spent many hours proof-reading this thesis and who provided thoughtful suggestions. Thanks also to Dr. Miles Copeland and the TRIO research engineers, in particular Tom Riley, for their advice and encouragement. Thanks to Dr. Calvin Plett for sharing his ideas throughout this thesis work. The assistance provided by Navid Foroudi and Maamoun Abou Seido contributed greatly to the work. Thanks are also due to Dennis Piamonte, who constantly and cheerfully shared the burden of preparing this and other related documents. The support and test-chip fabrication services of the Canadian Microelectronics Corporation (CMC) and Northern Telecom Electronics (NTE) are acknowledged as well.

I would like to express my most sincere thanks to the Canadian International Development Agency (CIDA) for its financial support throughout my period of study in Canada. Without this scholarship, my dream would not have been possible.

Finally, I also extend my love and thanks to my parents who have always supported me in everything I ever attempted. 


\section{Table of Contents}

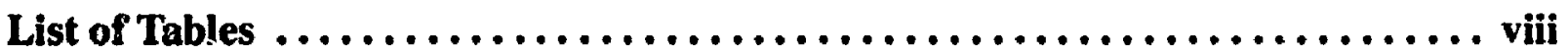

List of Figures........................................ ix

List of Symbols and Abbreviations $\ldots \ldots \ldots \ldots \ldots \ldots \ldots \ldots \ldots \ldots \ldots \ldots \ldots$ xii

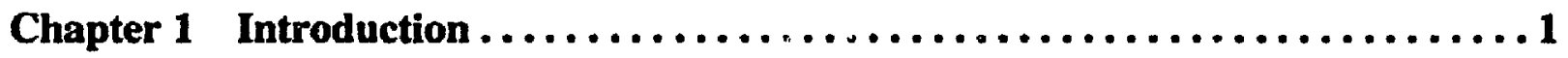

Chapter 2 Frequency Synthesizer for Mobile Transceiver $\ldots \ldots \ldots \ldots \ldots \ldots \ldots$

2.1. Types of frequency synthesis techniques...................

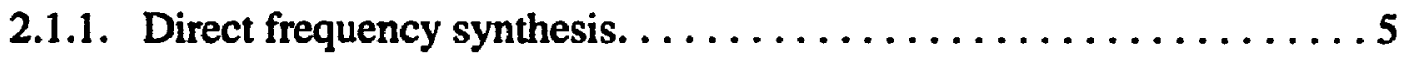

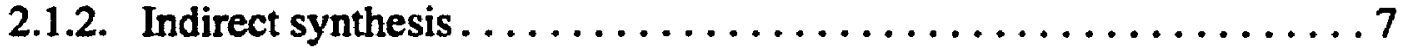

2.2. High-resolution, fast-switching PLL synthesizers. $\ldots \ldots \ldots \ldots \ldots \ldots \ldots 8$

2.2.1. Multiloop and hybrid synthesizer $\ldots \ldots \ldots \ldots \ldots \ldots \ldots \ldots \ldots . \ldots$

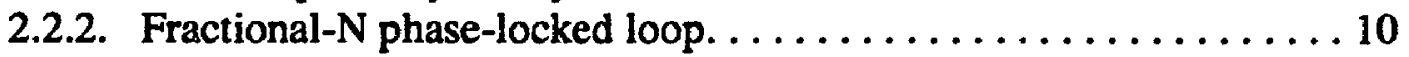

2.3. Monolithic PLL frequency synthesizer $\ldots \ldots \ldots \ldots \ldots \ldots \ldots \ldots \ldots 12$

2.3.1. The issue of monolithic implementation............... 12

2.3.1.1. Technology limitations .................. 13

2.3.2. Frequency synthesizer implementation technology $\ldots \ldots \ldots \ldots 17$

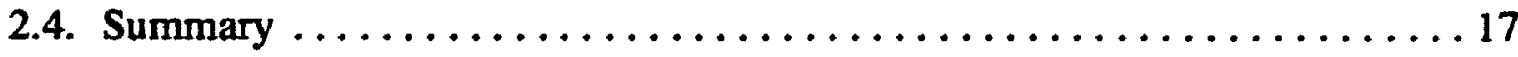

Chapter 3 The Realization of High-Speed PLL Components in CMOS Process 19

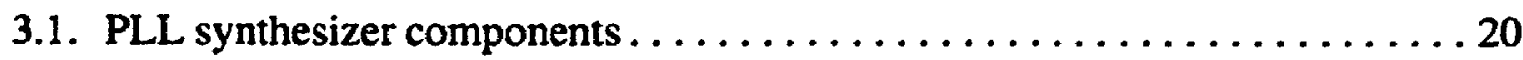

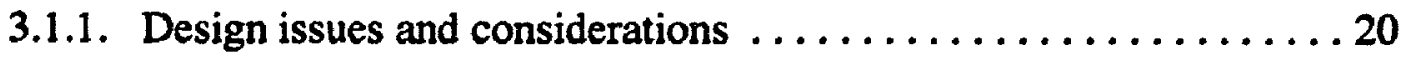

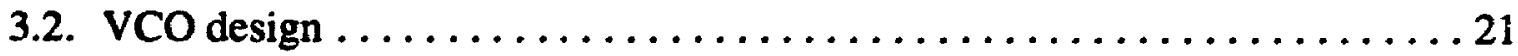

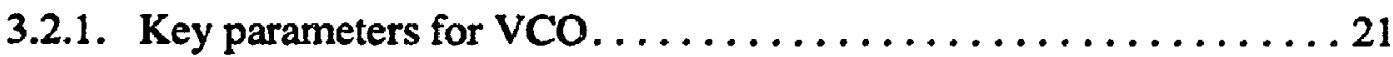

3.2.1.1. High spectral purity $\ldots \ldots \ldots \ldots \ldots \ldots \ldots \ldots \ldots 22$

3.2.1.2. Frequency of operation and power consumption $\ldots \ldots 22$

3.2.1.3. Suitability for circuit integration. . . . . . . . . . 23

3.2.2. Monolithic VCO design...................... 23

3.2.2.1. LC tank or tuned oscillators ............... 23

3.2.2.2. Multivibrator or relaxation based oscillators ........24

3.2.2.3. Comparison of bipolar ECOs and MOS relaxation

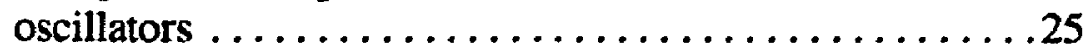

3.2.2.4. The phase noise generation mechanism ..........29 
3.2.3. Current VCO design......................... 30

3.2.4. CMOS oscillator design. ........................ 31

3.2.4.1. Speed limits. . . . . . . . . . . . . . . . . . . 31

3.2.4.2. Noise considerations................... 35

3.2.4.3. $\mathrm{Q}$ of CMOS relaxation oscillators $\ldots \ldots \ldots \ldots \ldots \ldots 35$

3.2.5. The analysis of CMOS oscillator in the time domain ........38

3.2.6. Techniques for the design of a low-noise oscillator $\ldots \ldots \ldots \ldots 4$ I

3.2.6.1. Regenerative feedback ................. 4l

3.2.6.2. Differential structure...................42

3.2.6.3. Circuit partitioning $\ldots \ldots \ldots \ldots \ldots \ldots \ldots \ldots \ldots 43$

3.3. The design of a PLL Synthesizer $\ldots \ldots \ldots \ldots \ldots \ldots \ldots \ldots \ldots \ldots \ldots$

3.3.1. Phase detector and loop filter. $\ldots \ldots \ldots \ldots \ldots \ldots \ldots \ldots \ldots 43$

3.3.1.1. CMOS phase detector.................43

3.3.1.2. Loop filter ....................... 45

3.3.1.3. Fully balanced phase-frequency detector and loop filter . . . 46

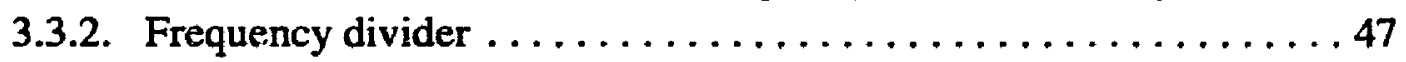

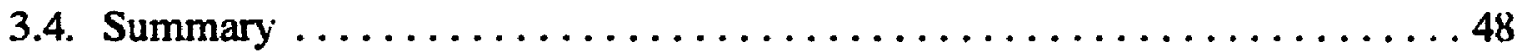

Chapter 4 The Design and Implementation of a CMOS Frequency Synthesizer 49

4.1. Linear ac model for frequency domain analysis $\ldots \ldots \ldots \ldots \ldots \ldots \ldots 50$

4.1.1. Proposed MOS model for ac analysis $\ldots \ldots \ldots \ldots \ldots \ldots \ldots \ldots 1$

4.1.2. Model derivation ........................ 52

4.2. High speed CMOS oscillator desiga $\ldots \ldots \ldots \ldots \ldots \ldots \ldots \ldots \ldots \ldots 5$

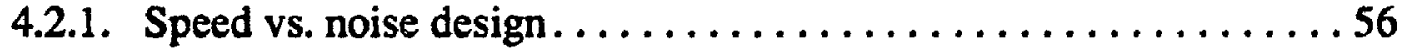

4.2.2. Modified ring oscillator $\ldots \ldots \ldots \ldots \ldots \ldots \ldots \ldots \ldots \ldots \ldots, \ldots \ldots \ldots$

4.2.3. Modified $D$-latch ring oscillator $\ldots \ldots \ldots \ldots \ldots \ldots \ldots \ldots \ldots 9$

4.2.4. Fully-differential ring oscillator $\ldots \ldots \ldots \ldots \ldots \ldots \ldots \ldots \ldots 6$

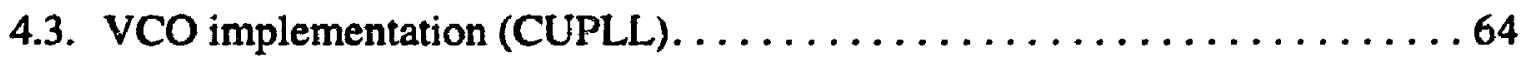

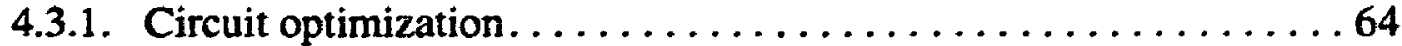

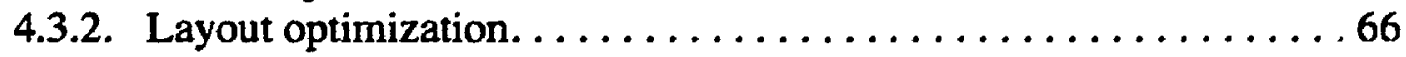

4.4. Simulation results.............................. 67

4.4.1. Post layout extraction simulation $\ldots \ldots \ldots \ldots \ldots \ldots \ldots 68$

4.4.2. Simulation for validation of the proposed ac MOS model . . . . . .73

4.4.3. Phase noise simulation. ..................... 76

4.4.4. Phase-frequency detector (PFD) with no dead-zone . . . . . . 80

4.5. Single-chip fractional- $\mathrm{N}$ frequency synthesizer design $\ldots \ldots \ldots \ldots \ldots \ldots 8$

4.5.1. Closed loop PLL component implementation........... 81

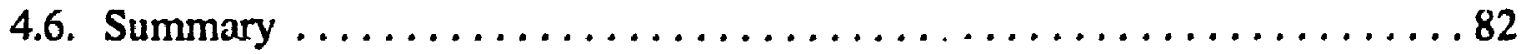


Chapter 5 Experimental Evaluation and Test Results $\ldots \ldots \ldots \ldots \ldots \ldots \ldots \ldots 84$

5.1. CUPLL experimental setups $\ldots \ldots \ldots \ldots \ldots \ldots \ldots \ldots \ldots \ldots \ldots \ldots \ldots$

5.1.1. CUPLL VCO-test setup. ........................ 85

5.1.2. VCO transfer characteristic .................... 86

5.1.3. VCO output spectra and waveform $\ldots \ldots \ldots \ldots \ldots \ldots \ldots . \ldots 8$

5.1.4. VCU power dissipation $\ldots \ldots \ldots \ldots \ldots \ldots \ldots \ldots \ldots \ldots . \ldots \ldots$

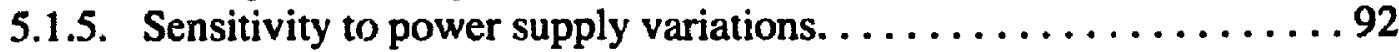

5.1.6. VCO phase noise measurement..................93

5.1.7. Test setup for verification of phase noise prediction technique. . . .96

5.1.8. Phase-frequency detcctor (PFL) test setup . . . . . . . . . . 99

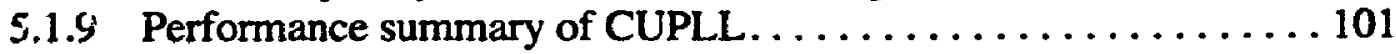

5.2. Single-chip CMOS fractional-N synthesizer (CUPL2) $\ldots \ldots \ldots \ldots \ldots 101$

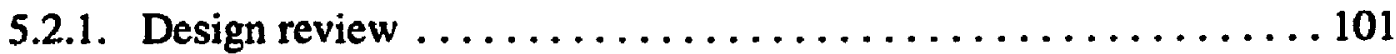

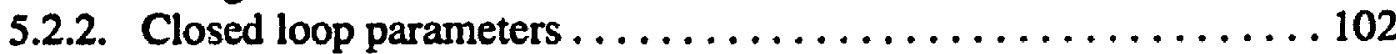

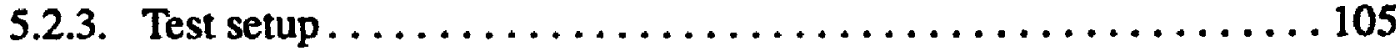

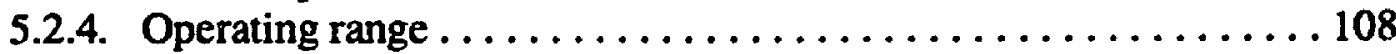

5.2.5. Fractional division.......................... 109

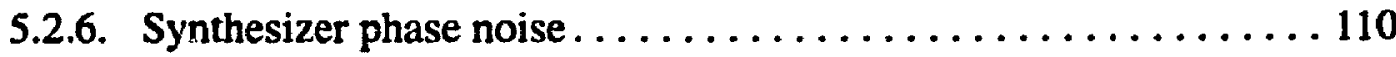

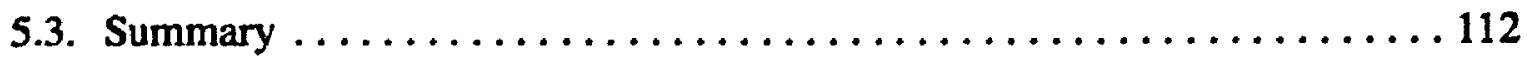

Chapter 6 Conclusion $\ldots \ldots \ldots \ldots \ldots \ldots \ldots \ldots \ldots \ldots \ldots \ldots \ldots \ldots \ldots \ldots \ldots$

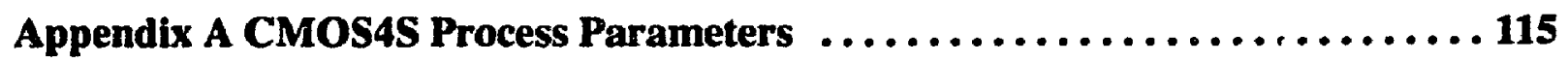

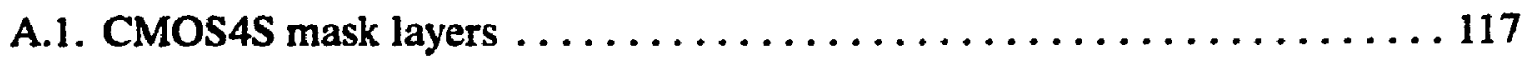

A.2. SPICE Level 3 Parameters. . . . . . . . . . . . . . . . . . . . 118

A.3. Other Electrical Parameters .......................... 119

Appendix B Physical Design: Chip Configurations, Layouts, and Device Sizes . . . 120

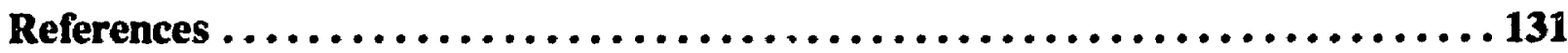

Bibliography $\ldots \ldots \ldots \ldots \ldots \ldots \ldots \ldots \ldots \ldots \ldots \ldots \ldots \ldots \ldots \ldots \ldots \ldots \ldots \ldots \ldots$ 


\section{List of Tables}

Table 2.1 Key technology parameters for digital wireless communications [10]... 5

Table 2.2 Comparison of frequency synthesis techniques $\ldots \ldots \ldots \ldots \ldots \ldots 12$

Tabl- $2.3 \quad$ Bipolar monolithic PLLs. . . . . . . . . . . . . . . . . . 14

Table $2.4 \quad$ BiCMOS and GaAs monolithic PLLs $\ldots \ldots \ldots \ldots \ldots \ldots \ldots \ldots$

Table $2.5 \quad$ MOS monolithic PLLs.......................... 6

Table 3.1 Collection of VCO implementations to date............... 30

Table 4.2 High-speed CMOS oscillators as parts of PLL applications and separate VCO circuits. . . . . . . . . . . . . . . . . . . 55

Table 4.3 VCO schematic level simulated results.................6. 65

Table 5.1 Summarized performance from the VCO design $\ldots \ldots \ldots \ldots \ldots 101$

Table $6.1 \quad$ Summarized results using different dividing ratios............ 109

Table A.1 CMOS4S mask layers........................ 117

Table A.2 SPICE Level 3 parameters $\ldots \ldots \ldots \ldots \ldots \ldots \ldots \ldots \ldots \ldots \ldots \ldots$

Table A.3 Resistance ............................... 119

Table A.4 Capacitance............................... 119 


\section{List of Figures}

Figure 2.1

Figure 2.2

Figure 2.3

Figure 2.4

Figure 2.5

Figure 2.6

Figure 2.7

Figure 2.8

Figure 3.1

Figure 3.2

Figure 3.3

Figure 3.4

Figure 3.5

Figure 3.6

Figure 3.7

Figure 3.8

Figure 3.9

Figure 3.10

Figure 3.11

Figure 3.12

Figure 3.13

Figure 3.14

Figure 3.15

Figure 3.16

Figure 3.17

Figure 3.18

Figure 3.19

Figure 3.20

Figure 4.1

Figure 4.2

Figure 4.3

Figure 4.4
Second generation cellular radio system concept. $\ldots \ldots \ldots \ldots \ldots \ldots \ldots \ldots \ldots$

Example of direct synthesis (a) Direct analog; (b) Direct digital . . . . . . . . . . . . 6

Phase-locked loop block diagram........................... 7

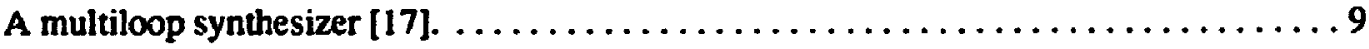

Phase-locked loop with direct digital synthesizer (DDS) $[15] \ldots \ldots \ldots \ldots \ldots \ldots \ldots$

Fractional-N frequency synthesizer. $\ldots \ldots \ldots \ldots \ldots \ldots \ldots \ldots \ldots \ldots \ldots$

Typical building blocks for a modern RF PLL frequency synthesizer...........13

Speed and power comparison graph of different PLL technologies. . . . . . . . 18

A VCO as a component of a fractional division PLL. .................. 20

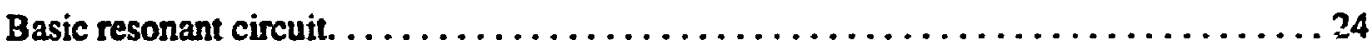

Multivibrator oscillators (a) Emitter coupled multivibrator, (b) Ring oscillator. . . . . . . 24

Generalized circuit for relaxation oscillator noise analysis. $\ldots \ldots \ldots \ldots \ldots \ldots \ldots 25$

Ring oscillator based on MOS inverter ring structure. . . . . . . . . . . . . 27

Ideal switching device and the jitter in actual relaxation oscillator. . . . . . . . . . 29

Ring oscillator and its representation in $\mathrm{RC}$ relaxation configuration. . . . . . . . 32

Simplified constant-curnent relaxation oscillator. . . . . . . . . . . . . . . . 33

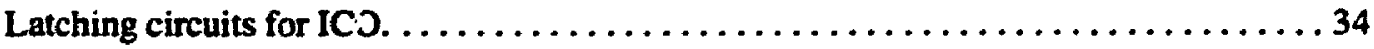

A high-speed ground capacitance modified emitter coupled oscillator. . . . . . . . 34

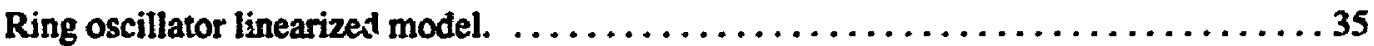

Linearized $1^{\text {st }}$ order model for three-stage multivibrator. ................. 36

Oscillator poles on complex frequency plan (a) Ideal case, (b) LC with losses (c) Multistage multivibrator. . . . . . . . . . . . . . . . . . . . . . . . . . 37

Timing diagram of $\mathbf{R}-\mathbf{C}$ relaxation oscillator. $\ldots \ldots \ldots \ldots \ldots \ldots \ldots \ldots \ldots \ldots \ldots$

MOS inverter with dynamic load capacitance in ring oscillator. $\ldots \ldots \ldots \ldots \ldots \ldots \ldots$

Regenerative network using a two-inverter latch $\ldots \ldots \ldots \ldots \ldots \ldots \ldots \ldots \ldots, \ldots \ldots \ldots$

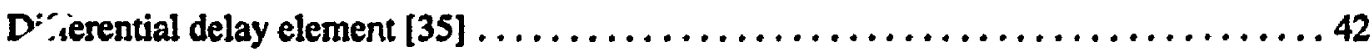

Phase-frequency detector with no dead zone. . . . . . . . . . . . . . . . 44

Choices of loop filter implementation. . . . . . . . . . . . . . . . . . . . . 46

Fully balanced configuration of phase detector and loop filter. . . . . . . . . . 47

(a) Basic block diag-am for representation of VCO in frequency domain. (b) Model of an injected noise current for actual analysis in oscillator circuit. . . . . . . . . . . . 51

Hspice equivalent circuit MOSFET [71] . . . . . . . . . . . . . . . . . 53

A proposed linear ac model for MOS devices in oscillator circuits. . . . . . . . . . 54

(a) Basic delay cell and (b) Dynamic NMOS inverter as variable inverting delay cell. . . . .57 
Figure 4.5

Figure 4.1

Figure 4.7

Figure 4.8

Figure 4.9

Figure 4.10

Figure 4.11

Figure 4.12

Figure 4.13

Figure 4.14

Figure 4.15

Figure 4.16

Figure 4.17

Figure 4.18

Figure 4.19

Figure 4.20

Figure 4.21

Figure 4:22

Figure 4.23

Figure 5.1

Figure 5.2

Figure 5.3

Figure 5.4

Figure 5.5

Figure 5.6

Figure 5.7

Figure 5.8

Figure 5.9

Figure 5.10

Figure 5.11

Figure 5.12

Figure 5.13

Figure 5.14

Figure 5.15
Short-channel effect in the MOSFET saturation mode and its suitable application for

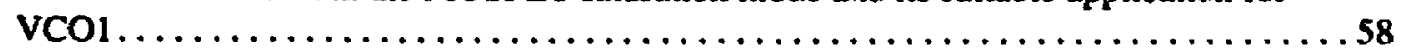

vCO1 simplified three-stage modified ring oscillator. $\ldots \ldots \ldots \ldots \ldots \ldots \ldots 59$

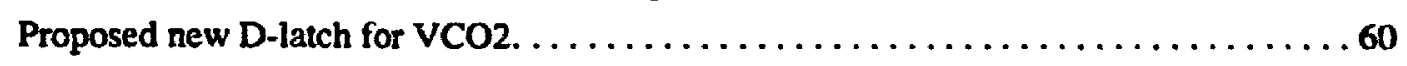

vCO2 modified D-latch ring oscillator. .....................60

CMOS implementation of emitter-coupled structure using double flip-flops. . . . . 62

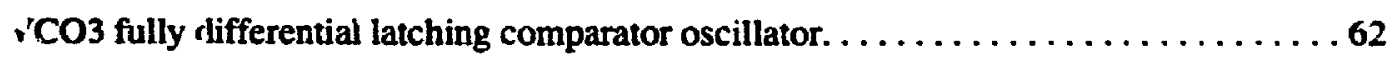

VCOs shown with its inherent regenerative circuits. . . . . . . . . . . . . 63

Simulated VCO outputs, dotted line are the signals probed at the output FADs. . . . 69

vCO transfer characteristics. $\ldots \ldots \ldots \ldots \ldots \ldots \ldots \ldots \ldots \ldots \ldots \ldots \ldots$

Effects of temperature variation on the VCO operating point. . . . . . . . . . . 72

(a) Oscillator's maximum frequency versus supply voltag's and (b) Power con-

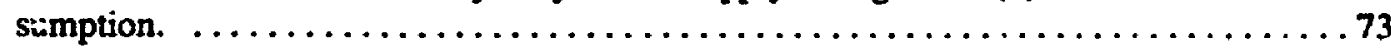

Simulation performed on developed ac MOS model using injected noise current

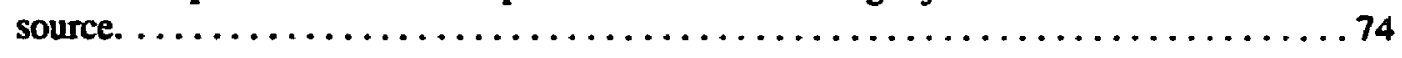

Frequency response of VCOl to noise using developed ac model $\ldots \ldots \ldots \ldots \ldots \ldots 5$

Frequency response of VCOs to noise using developed ac mudel. . . . . . . . . 76

Equivalent circuits for a simulated injected noise current and its actual power supply noise superposition in the measurement stage. $\ldots \ldots \ldots \ldots \ldots \ldots \ldots \ldots \ldots \ldots 77$

Simplified simulation setup for phase noise prediction technique. $\ldots \ldots \ldots \ldots \ldots 78$

Simulated VCO spectra demonstrating phase noise correlations. . . . . . . . . . 79

Simulated phase-frequency detector characteristics, fin $=10 \mathrm{MHz}, 25 \mathrm{MHz}$ and $50 \mathrm{MHz} . \ldots 80$

Single-chip implementation of the CMOS frequency synthesizer, CUPL2. $\ldots \ldots \ldots 82$

CUPLL VCOs' test setup $\ldots \ldots \ldots \ldots \ldots \ldots \ldots \ldots \ldots \ldots \ldots \ldots \ldots \ldots \ldots$

VCO Voltage-to-frequency transfer characteristic curves. $\ldots \ldots \ldots \ldots \ldots \ldots \ldots \ldots 7$

Spectra traces of VCO1 and VCO2 outputs at $1 \mathrm{kHz}$ resolution bandwidth. . . . . . 88

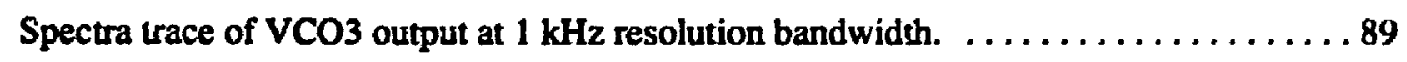

Oscilloscope traces for VCO waveforms. . . . . . . . . . . . . . . .

Power dissipation as a function of oscillating frequency. . . . . . . . . . . . . 91

Frequency of oscillation as a runction of supply voltage. $\ldots \ldots \ldots \ldots \ldots \ldots \ldots 2$

Test bed \& setup for VCO phase noise characterization. . . . . . . . . . . . . 94

Measured VCO1, VCO2, VCO3 residue FM/SSB phase noise using HP3804A. . . . . . 95

Test setup for verification of phase noise prediction technique. . . . . . . . . . 96

VCOi spectra trace at $900 \mathrm{MHz}$ before and after added noise. . . . . . . . . . 97

VCO2 Spectra trace before and after added noise. . . . . . . . . . . . . 98

Modulated spectra output of VCO3, demonstrating best noise rejecting capability. . . . 98

Test setup for measurement of phase-frequency detector characteristic. . . . . . . . 99

Measured phase-frequency characteristic. .100 
Figure 5.16 (a) Loop filter, single ended, (b) differential configuration with prefiltering. . . . . . . 104

Figure 5.17 Test setup for the single-chip fractional-N CMOS frequency synthesizers. . . . . . 106

Figure 5.18 Setup configuration for measuzement of synthesizer operating frequency and phase noise. . . . . . . . . . . . . . . . . . . . . . . . . . . . . . . 108

Figure 5.19 Modification for a new fractional dividing ratıo. $\ldots \ldots \ldots \ldots \ldots \ldots \ldots \ldots \ldots \ldots$

Figure $5.20 \quad$ PLL spectral outputs at $850-\mathrm{MHz}_{\mathrm{z}}$ center frequency. $\ldots \ldots \ldots \ldots \ldots \ldots \ldots \ldots \ldots \ldots$

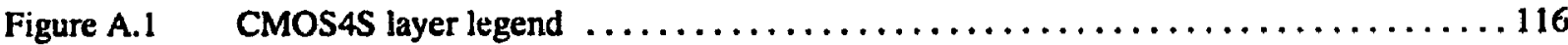

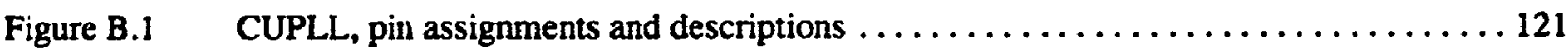

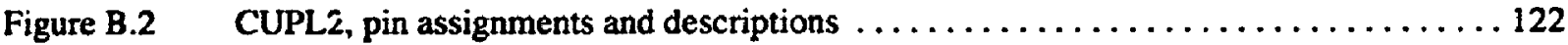

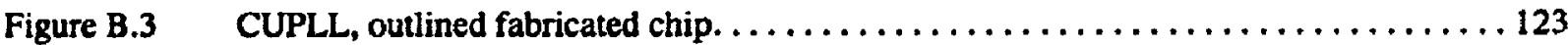

Figure B.4 CUPL2 (ICACUPL2), outlined fabricated chip $\ldots \ldots \ldots \ldots \ldots \ldots \ldots \ldots \ldots \ldots 124$

Figure B.5 VCO1, three-stage modified ring oscillator $\ldots \ldots \ldots \ldots \ldots \ldots \ldots \ldots \ldots \ldots \ldots$

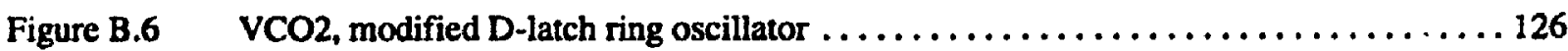

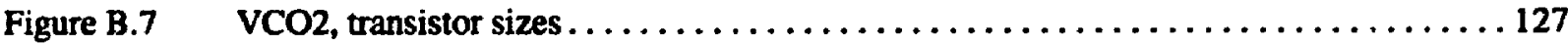

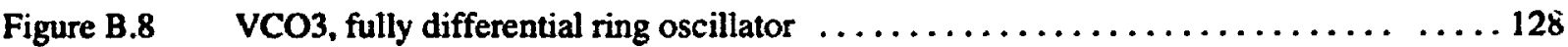

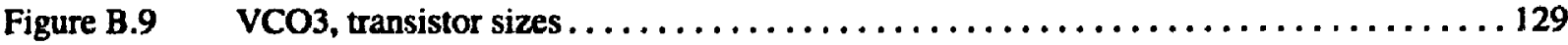

Figure B.10 Fully balanced phase-frequency detector with no dead zone $\ldots \ldots \ldots \ldots \ldots \ldots \ldots 130$ 


\section{List of Symbols and Abbreviations}

\begin{tabular}{|c|c|}
\hline$\frac{1}{=}$ & 0-volt reference, Gnd \\
\hline & Supply voltage, Vad \\
\hline & n-MOSFET transistor \\
\hline$-\infty$ & p-MOSFET transistor \\
\hline$-4 r$ & Resistor \\
\hline & Potentiometer \\
\hline & Capacitor \\
\hline & Inverter \\
\hline & Buffer \\
\hline & Exclusive OR gate, XOR \\
\hline & NOR gate \\
\hline & Diode \\
\hline & Zener Diode \\
\hline & Operational amplifier \\
\hline
\end{tabular}


BiCMOS Bipolar CMOS

CMOS Complimentary Metal Oxide Semiconductor

CT2 Cordless telephone standard

DAC Digital-to-analog convertor

dc

Direct current

DDS Direct digital synthesis

DECT Digital European Cordless Telepłone System

GSM Global System for Mobile Communication

IS-54 Electronic Industry Association Interim Standard 54

PFD Phase-frequency detector

PLL Phase-locked loop

Q Quality Factor

RF Radio frequency

SAW Surface Acoustic Wave

Si Silicon

SPICE a circuit simulator

TDMA Time-division multiple access

TRIO Telecommunication Research Institute of Ontario

VCO Voltage-controlled oscillator

W/L Width-to-length ratio of a transistor 
The information used in this thesis comes in part from the research program of Dr. Tadeusz A. Kwasniewski and his associates in the VLSI in Communications group. The research results appearing in this thesis represent an integral part of the ongoing research program. All research results in this thesis including tables, graphs and figures but excluding the narrative portions of the thesis are effectively incorporated into the research program and can be used by Dr. Kwasniewski and his associates for education and research purposes, including publication in the open literature with appropriate credits. Matters of intellectual property may be pursued collaboratively with Carleton University and Dr. Kwasniewski where and as appropriate. 


\section{Chapter 1}

\section{Introduction}

The rapid growth in the use of digital mobile radio and portable telephones has been accomplished by steady reductions in cost, terminal size, and power consumption. For the terminal manufacturers to remain competitive, digital mobile terminal designs remain a very challenging task driven by consumer-market demands. The key technology issues to be addressed relate to minimum complexity architecture, low power, and maximum use of very large-scale integrated circuits (VLSI).

Currently, the implementation of digital mobile radio components is of a strong research interest. Both CMOS and BiCMOS implementation paths are considered. The reported circuits to date, do however, suffer from high complexity and high power consumption $[1,2,3,4]$. Topics relating to the components of a frequency synthesizer are the subject of ongoing research within the Department of Electronics, VLSI in Communications Research Group at Carleton University. Specifically, work has been done on a dual-modulus frequency divider (N. Foroudi [5]), a phase-frequency detector (T. Pantazopoulos [6]), and in particular a full fractional-N PLL $\Delta \Sigma$ modulated frequency 
synthesizer (T. Riley [7], T. Kenny [8]), which precede research reported in this thesis. The contribution of this thesis is an all CMOS implementation of a PLL frequency synthesizer.

The thesis is organized in the following manner.

Chapter 2 reviews the existing frequency synthesis techniques and their VLSI implementations along with a brief performance analysis of phase-locked loop (PLL) synthesizers constructed in different implementation specific silicon technologies. The limitations of the integrated frequency synthesizer approach are also presented.

Detailed design considerations for a high-frequency low-phase-noise voltage controlled oscillator (VCO) as an integral part of a PLL circuit component are studied in Chapter 3. The design issues and analysis of CMOS multivibrators in the time domain, based upon ring oscillator structures, are discussed and suitatle structures for CMOS VCOs introduced. As well, the design of a CMOS indirect frequency synthesizer is included.

Chapter 4 focuses on techniques for achievement of high-speed and low-noise VCO implementation. The ac MOSFET model for the design of CMOS oscillators, developed by the author and co-researchers, Maamoun Abou Seido and Dr. Tadeusz A. Kwasniewski, is reported. The initial work on the model as reported in this thesis is now further pursued within the VLSI in communications research group. Three ViO architectures, considered suitable for various speeds and noise performance, are presented along with an evaluation of the proposed model's accuracy and a phase noise simulation technique. A prototype single-chip implementation consisting of an all CMOS fractionalN PLL frequency synthesizer, excluding the loop filter, is then proposed.

Test results for two fabricated chips are reported and discussed in Chapter 5. The final chapter summarizes the thesis and suggests directions for future work. 


\title{
Chapter 2
}

\section{Frequency Synthesizer for Mobile Transceiver}

\author{
A block diagram of a second generation digital mobile radio [9] working around 900 \\ $\mathrm{MHz}$ or $1.9 \mathrm{GHz}$ is shown in Figure 2.1. The frequency synthesizer embedded in the \\ circuit front end provides the RF programmability required in the transmission and \\ reception of the digital baseband signals. With increasing use of wireless terminals, the \\ bandwidth per user is reduced, as can be seen in Table 2.1 [10]. Modulation frequency \\ resolution and channel switching speed have become the most important parameters for \\ the frequency synthesizer in high spectrum efficiency digital mobile radio systems.
}

The design for frequency programmability for a hand-held terminal should begin with an examination of the design parameters required for a frequency synthesizer. These requirements $[11,12]$ include: 
" Fast settling time

- Low phase noise

- Low power consumption

- Small size (monolithic implementation)

- Good spurious frequency performance.

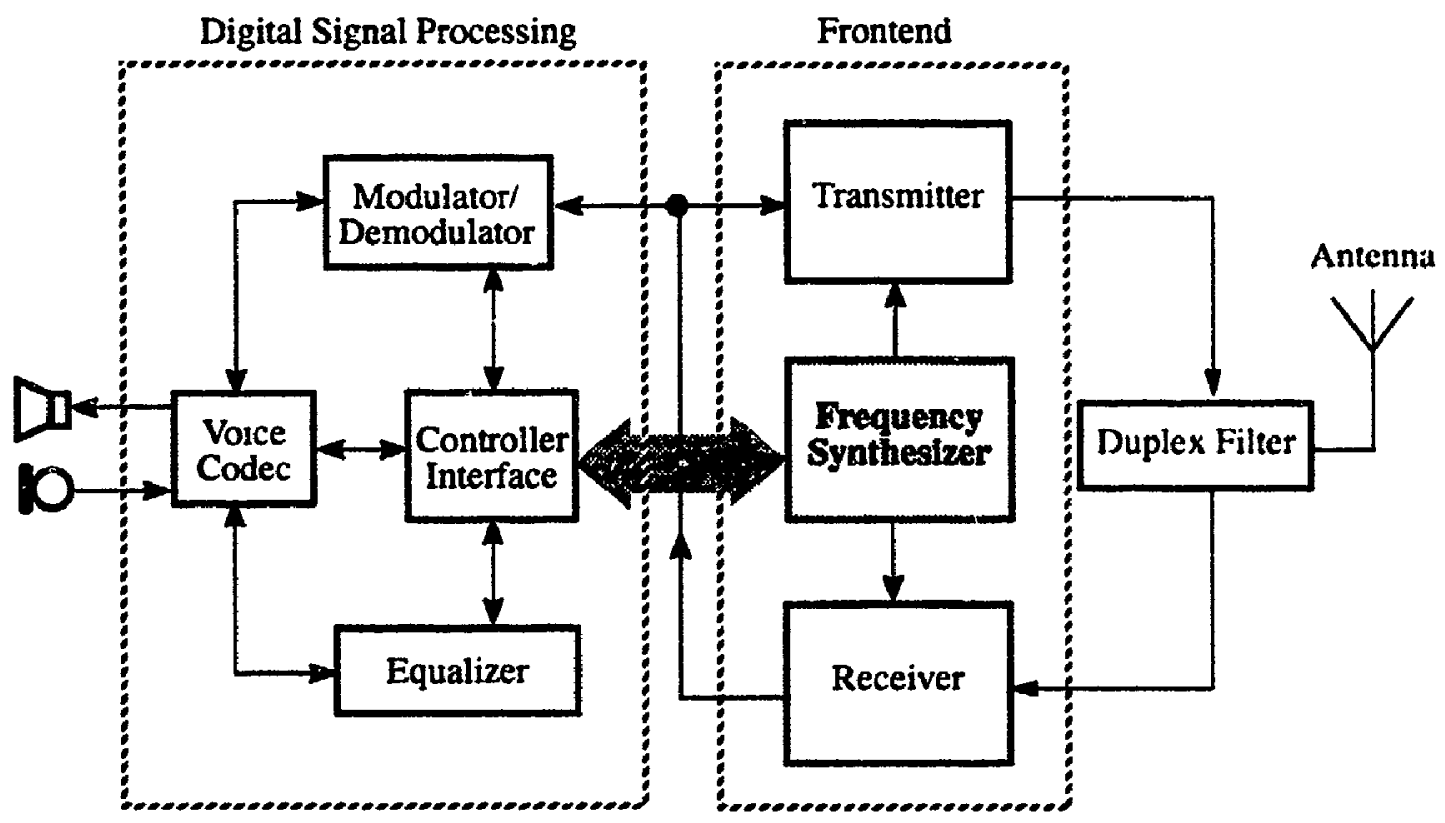

Figure 2.1 Second generation cellular radio system concept.

From the standpoint of size, cost, and performance, greater integration of radio components into silicon is a vital step.

In this chapter, modern frequency synthesis techniques are reviewed. A comparison of current high-frequency synthesizer architectures implemented with different technologies is provided. Special emphasis is placed on indirect synthesis techniques and their implementations since they show the most promise of meeting all the required performance parameters. Finally, the ideal solution that is viable within today's technologies is proposed. 


\begin{tabular}{|c|c|c|c|c|c|c|c|}
\hline Specification & IS-54 & GSM & $\mathrm{CT}-2$ & DECT & DCS1800 & JDC & PHP \\
\hline Frequency (MHz) & $824-894$ & 890.960 & $864-868$ & $1,880-1,900$ & $1,700-1,880$ & 80011.500 & 1.900 \\
\hline Access method & $\begin{array}{l}\text { TDMA } \\
\text { FDMA }\end{array}$ & $\underset{\text { FDMA }}{\operatorname{TUMA}}$ & FDMATTDD & TDMA/TDD & $\begin{array}{l}\text { TDMA } \\
\text { FDMA }\end{array}$ & $\begin{array}{l}\text { TDMA } \\
\text { FDMA }\end{array}$ & TDMATTDD \\
\hline Modulation & $\pi / 4$ DQPSK & GMSK & MSK & GMSK & GMSK & $\pi / 4$ DQPSK & $\pi / 4$ DOPSK \\
\hline Bit rate (kbits/s) & 48.6 & 270.8 & 72 & 1152 & 270 & 42 & 384 \\
\hline Voice coding & VSELP & RPE-LPT & ADPCM & ADPCM & RPE. .PT & VSELP & ADPCM \\
\hline Power output (W) & 0.6 .1 .2 .3 & 2.5 .8 .20 & $10 \mathrm{mw}$ & $250 \mathrm{mw}$ & 0.25 .1 .0 & 0.3 .0 .8 .2 & $10 \mathrm{mw}$ \\
\hline Carriers & 832 & 125 & 40 & 10 & 375 & $T B D$ & TDB \\
\hline Carriers spacing (cllz) & 30 & 200 & 100 & 1728 & 200 & 25 & 300 \\
\hline Channels/Carrier & 3.6 & 8 & 1 & 12 & 8 & 3 & 4 \\
\hline
\end{tabular}

Table 2.1 Key technology parameters for digital wireless communications [10].

\subsection{Types of frequency synthesis techniques}

The first step in designing frequency synthesizers is to determine which of the most commonly used synthesis techniques best satisfies performance requirements (i.e., direct analog, direct digital or phase-locked loop).

\subsubsection{Direct frequency synthesis}

Direct analog synthesis is the generation of frequencies from one or more reference frequencies using analog techniques through a combination of multipliers, dividers, mixers and bandpass filters (Figure 2.2. a). While this method can provide fine resolution, fast switching, and low noise, the hardware requires too much space and power consumption for use in portable receivers. Moreover, because the frequency range of interest is nearly an octave, the spurious frequencies generated by this approach would not meet the established specifications [12].

Direct digital synthesizers as shown in Figare 2.2.b produce signais with fine frequency resolution, fast settling speed, and good spurious response. In VLSI form, these 
synthesizers offer relatively small size and low power consumption. Their limitation is the limited hightsi output frequencies obtainable while maintaining low power consumption and reasonable spurious performance. For example, a 150-MHz frequency synthesizer with $-90 \mathrm{dBc}$ spurious performance implemented in a $1.25-\mu \mathrm{m}$ CMOS, dissipates $950 \mathrm{~mW}$ at $100 \mathrm{MHz}$ [2]. Therefore, with the current available technologies, it would not be possible to implement frequency synthesizers directly with direct digital synthesis and still meet all requirements.

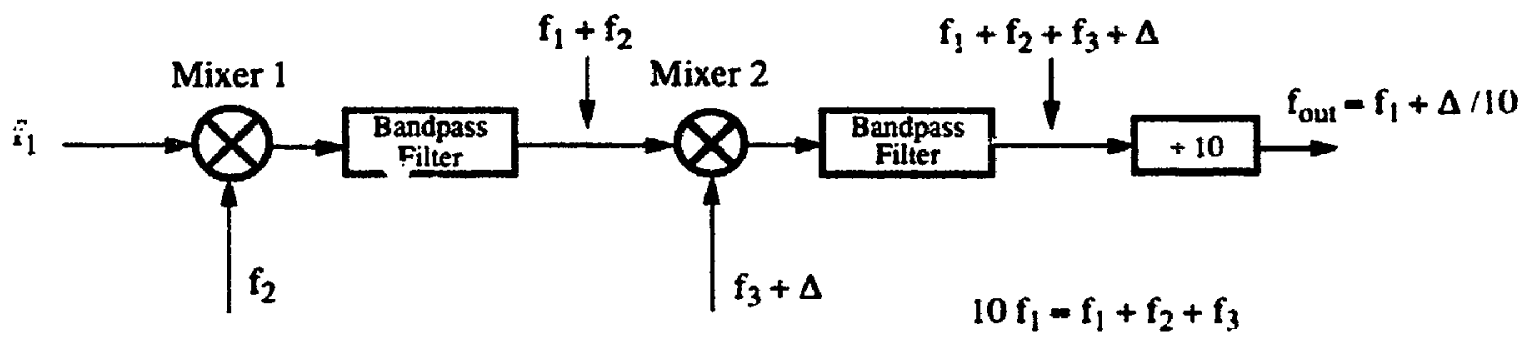

a) Direct Analog Synthesis

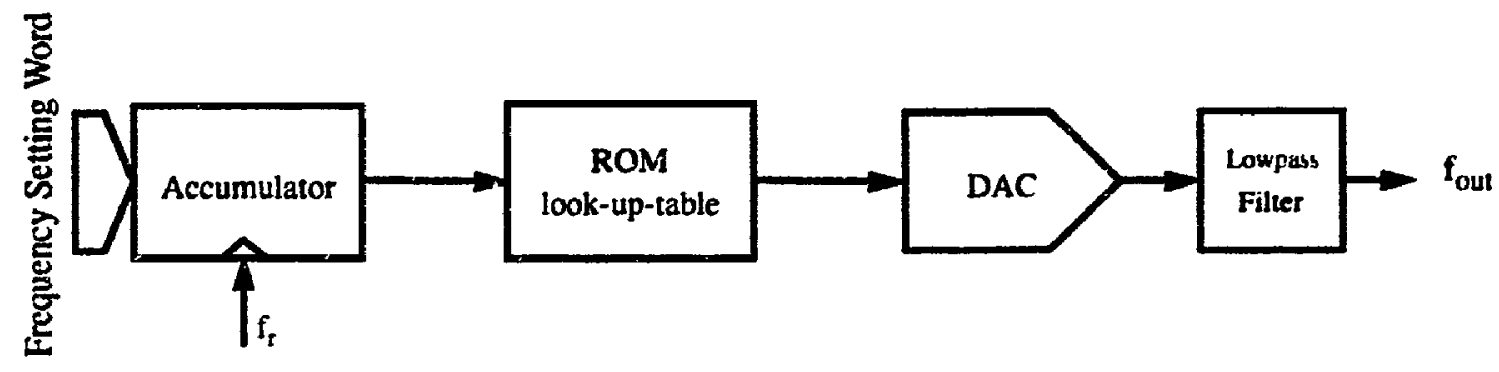

b) Direct Digital Synthesis

Figure 2.2 Example of direct synthesis (a) Direct analog; (b) Direct digital. 


\subsubsection{Indirect synthesis}

On the other hand, indirect frequency synthesizers employ a secondary oscillator controlled by a phase-locked loop (PLL) or a frequency-locked loop to generate the output frequency as shown in Figure 2.3. This type of synthesizer is relatively simple and inexpensive in comparison to a direct synthesizer. Because of their small-size and low power consumption, nearly all of today's miniature radio transceivers rely on phaselocked loop synthesizers $[11,13]$. The basic form of the PLL consists of a voltagecontrolled oscillator (VCO), a frequency divider, a phase detector, and a lowpass filter.

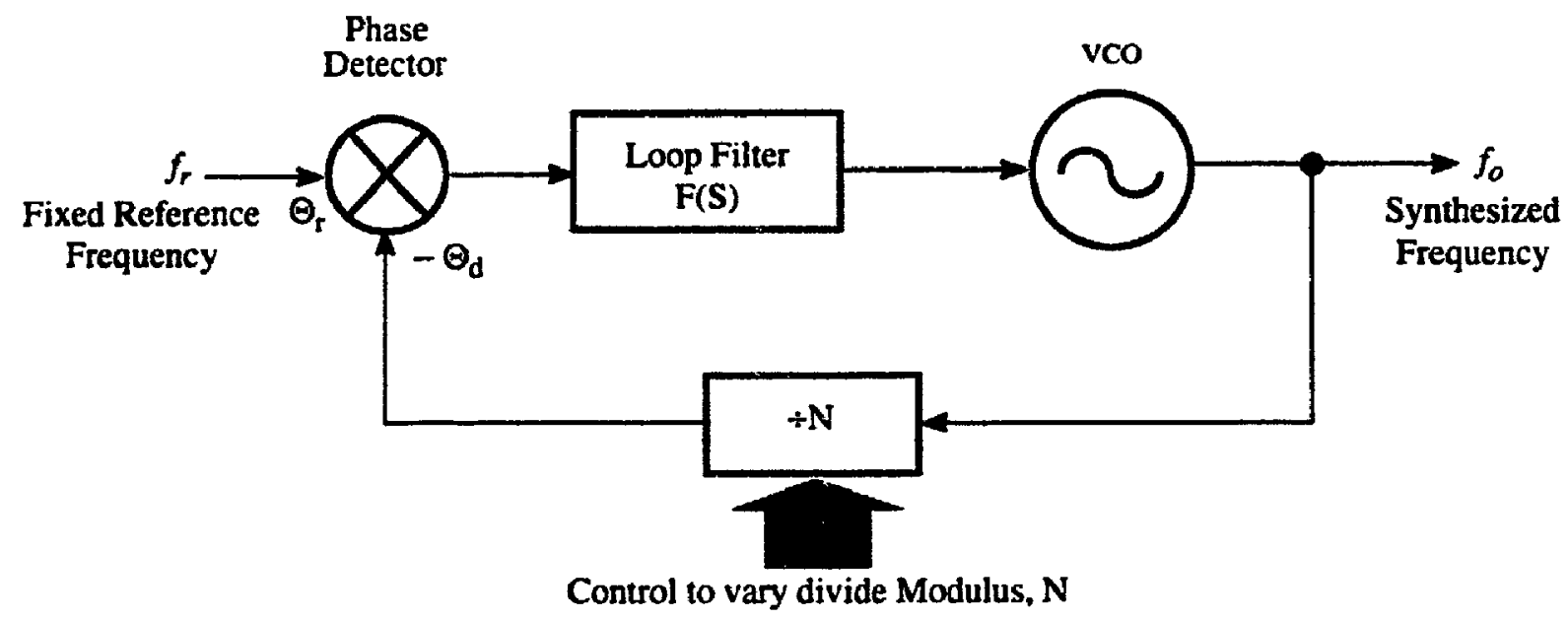

Figure 2.3 Phase-locked loop block diagram.

The VCO generates the synthesized frequency according to a dc level at its input. Such an arrangement allows the possibility of slaving a synthesized frequency to a spectrally pure. lower reference frequency. In the lock condition, the VCO output frequency $f_{o}$ is related to the reference frequency $f_{r}$ by $f_{o}=N \cdot f_{r}$ where $N$ is the divider modulus. This indicates that the smallest frequency increment generated by the loop is equal to the reference frequency. 
Typically, $\mathrm{N}$ would be a large integer, so as to give a narrow channel spacing capability for the synthesized frequency. However, the switching speed of the PLL is a function of the loop bandwidth and closed-loop frequency response. Since the bandwidth is always smaller than $f_{r}$ in order to provide a stable correction signal to the $\mathrm{VCO}$, faster switching speeds renu're higher reference frequencies and therefore higher loop bandwidths. It is apparent from $[11,12,14]$ that the loop design parameters cannot be chosen to optimize all aspects of circuit performance. The result is a trade-off between narrow bandwidths for maximum filtering and wide bandwidths to provide short risetimes for ast tracking of the input signal.

\subsection{High-resolution, fast-switching PLL synthesizers}

In a conventional single loop PLL, as shown in Figure 2.3, the frequency step is determined by the reference frequency. Attempts to reduce the reference frequency in order to inclease the frequency resolution would result in an increased division ratio $(N)$. A narrower loop bandwidth would also be required, resulting in large reference feedthrough and lower switching speed. The alternatives most frequently applied to the fixed division ratio approach are discussed in the following subsections.

\subsubsection{Multiloop and hybrid synthesizer}

The use of a multiloop PLL synthesizer is particularly advantageous for a frequency synthesizer requiring small frequency increments. By using an auxiliary PLL in such a way as to tune the auxiliary VCO over a small frequency range, the synthesizer output frequency can be changed with a fine resolution step. An example of a multiloop synthesizer used in a typical receiver-conversion scheme is shown in Figure 2.4.

The synthesizer operation is based upon two PLLs, A and B. Assuming that both use the same reference frequency of $99 \mathrm{MHz}$, loop A generates the $9900 \mathrm{~Hz}$ incremenis and loop B the $10,000 \mathrm{~Hz}$ changes in output frequency. The final output $f_{\text {out }}$ can be obtained when the two loops are summed together in the output mixer as follows: 


$$
f_{\text {out }}=N \times f_{\text {refl }}-M \times f_{\text {ref } 2}+f_{\text {offset }}
$$

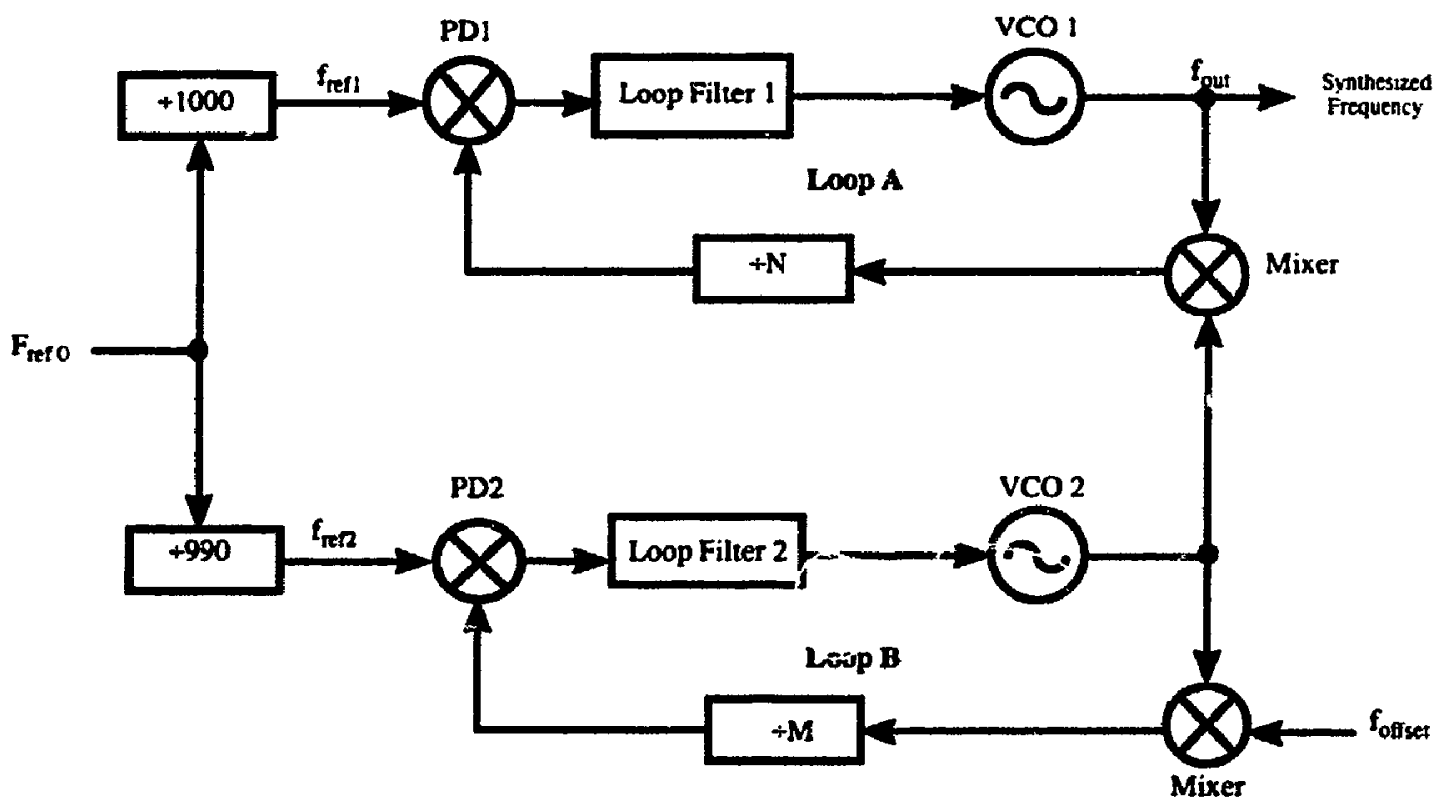

Figure 2.4 A multiloop synthesizer [17].

Since the response time of the frequency synthesizer is determined by the individual response times of loops A and B, the loop bandwidth used for each loop can be fairly wide. Consequently, the overall switching time is not significantly degraded. By using this method, it is possible to achieve a frequency resolution of $100 \mathrm{~Hz}$ and obtain fast switching speed at the same time. This technique is widely accepted for microwave synthesizers where broadband PLLs with fast switching times are necessary.

Next, the hybrid synthesizer that consists of a direct digital synthesizer (DDS) and a PLL loop shown in Figure 2.5 is discussed. A DDS, together with an upconverter, is used to generate an offset frequency that is mixed with the VCO output [11, 15]. This method provides an improved tuning resolution and switching time since fine steps are produced by changing the output of the DDS. The DDS synthesizer can typically switch in much less than a microsecond. 


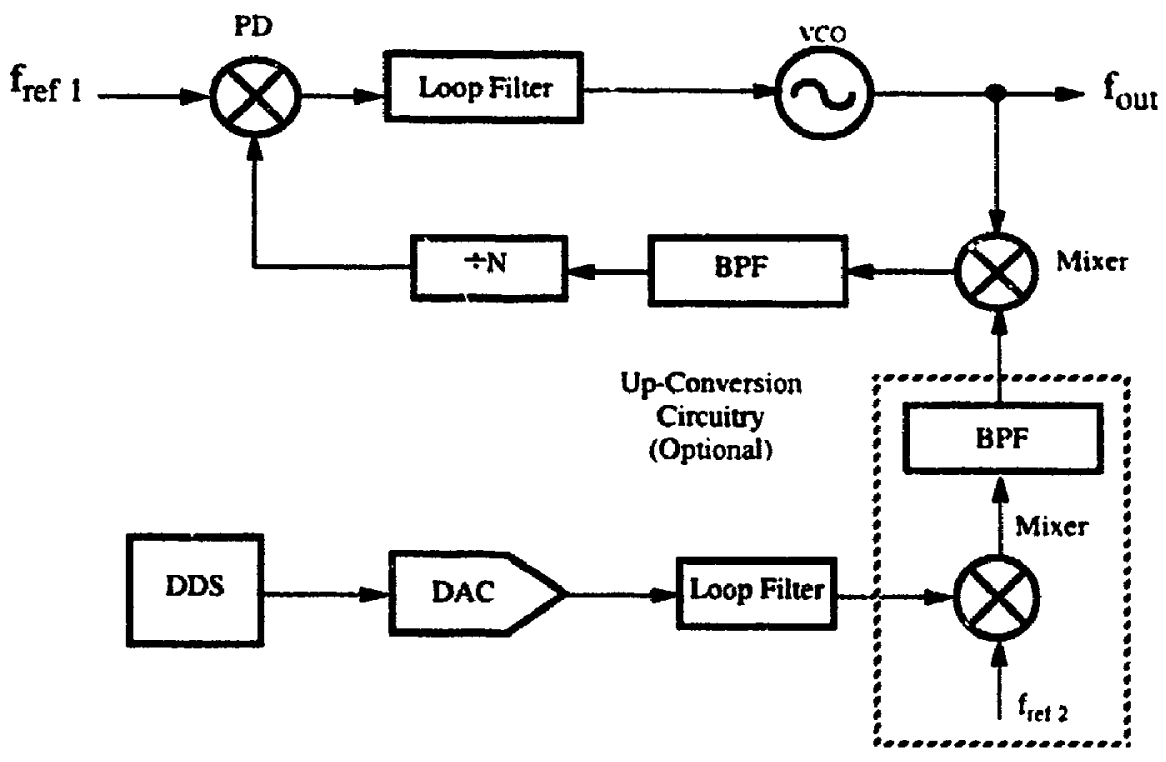

Figure 2.5 Phase-locked loop with direct digital synthesizer (DDS) [15].

The key disadvantage of using both synthesizers is their high cost in hardware complexity and poor spurious signal performance. In addition, they include mixers and filters whose monolithic implementation is difficult and which have high power consumption.

\subsubsection{Fractional-N phase-locked loop}

As pointed out earlier, the response time of the PLL depends largely on the loop bandwidth. Consequently, to obtain fast switching it is necessary to reduce the loop response time by widening the loop bandwidth. One possible way of achieving a fine frequency step without either lowering the reference frequency or considerably increasing the divide. ratio, is to make use of a fractional division approach. The basic fractional-N phase-locked loop technique involves use of a high-speed frequency divider working in conjunction with a low-speed programmable counter whose division ratio is the sum of an integer and a fraction of an integer. The fractional division is substituted for the fixed programmable divider of conventional PLL synthesizers as shown in Figure 2.6. This approarh is also known as Variable-Modulus Dividing [11] or the Pulse Swallowing Technique $[12,16]$. References [11] and [16] provide more details on the operation of this synthesizer, which is summarized briefly here. 


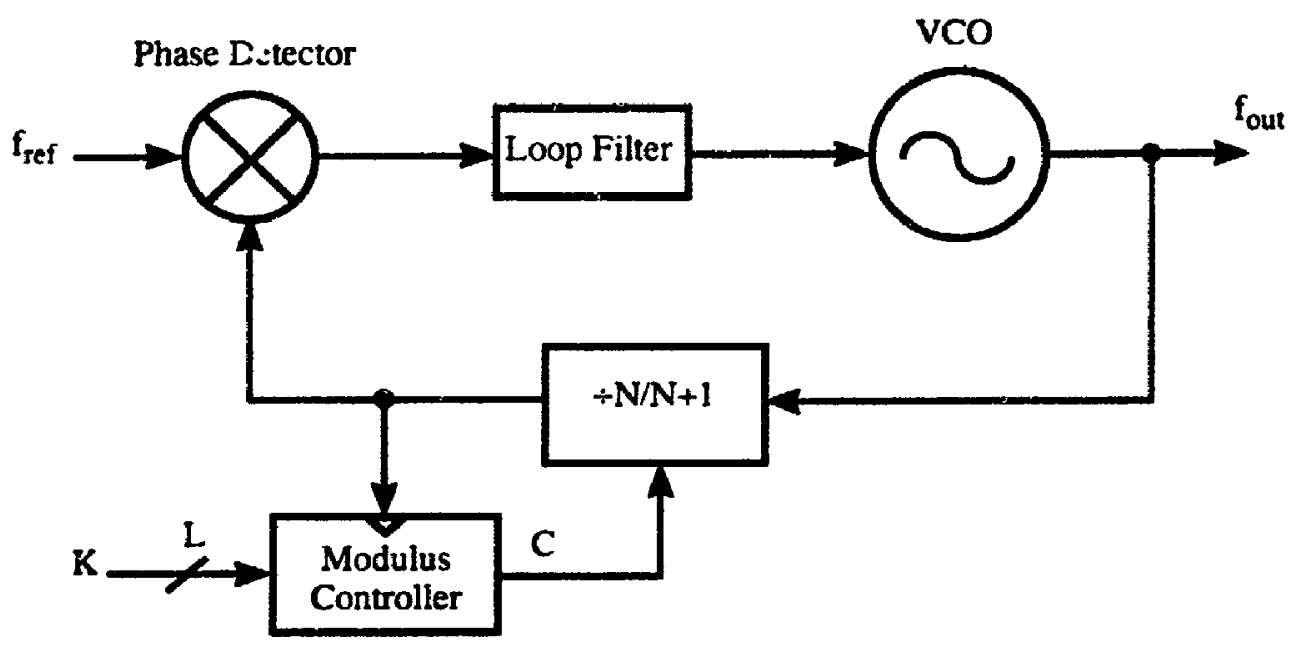

Figure 2.6 Fractional-N frequency synthesizer.

The dual modulus divider divides by $\mathrm{N}$ or $\mathrm{N}+1$ when the modulus control signal is at logic 1 or logic 0 respectively. A digital phase accumulator can be used as the modulus controller with the accumulator content initially set to zero. The system starts with the dual modulus divider dividing by $\mathbf{N}$. The accumulator input, $K$, is added to the accumulator for every cycle of the reference until overflowing occurs, resulting in carry signal $\mathrm{C}$ outputting to the dual modulus divider to divide by $\mathrm{N}+1$ for one cycle. The sequence then begins counting again. The resulting average output frequency is given by

$$
f_{\text {out }}=\left(N+\frac{K}{2^{L}}\right) \times f_{\text {ref }}
$$

Equation 2.2 implies that a high resolution can be achieved by the choice of accumulator length, $L$, and the programmable input value, $K$. The major advantage of this technique is the high frequency resolution that can be attained while still maintaining good performance, allowing for the usc of low-noise CMOS logic for programmable dividers.

The effect of changing the divider ratio between two integers, $\mathrm{N}$ and $\mathrm{N}+1$, causes periodic phase discontinuity at the output of the frequency divider. These phase fluctuations, referred to as phase jitter. are detected by the phase detector and produce spurs at the phase 
detector output. This signal, in turn, can propagate to the VCO via the loop filter and cause unwanted spurious spike spectral components around the synthesized frequency.

This inherent drawback of the fractional- $\mathrm{N}$ synthesizer have been treated by Jaquet [17]. Among them, the $\Delta \Sigma$ modulator method appears to be the most effective in terms of low-power and low-cost digital VLSI implementation [7].

\subsection{Monolithic PLL frequency synthesizer}

Table 2.2 summarizes the properties of different frequency synthesis techniques as reported by Riley et al [7]. As previously discussed, among them, the fractional- $N$ synthesis method is theoretically best suited to single-chip implementation.

\begin{tabular}{|c||c|c|c|c|c|}
\hline Synthesis Technique & $\begin{array}{c}\text { Single- } \\
\text { Chip } \\
\text { Implement } \\
\text { ation }\end{array}$ & $\begin{array}{c}\text { Maximum } \\
\text { Frequency }\end{array}$ & $\begin{array}{c}\text { Power Con- } \\
\text { sumption } \\
\text { (at the same } \\
\text { frequency) }\end{array}$ & Resolution & $\begin{array}{c}\text { Settling } \\
\text { Time }\end{array}$ \\
\hline \hline Direct Anglog & Difficult & $\sqrt{ }$ & $\sqrt{ }$ & $\sqrt{ }$ & $\sqrt{ }$ \\
\hline Direct Digital & $\sqrt{ }$ & Low $\left(F_{\text {clock }} / 2\right)$ & $\sqrt{ }$ & $\sqrt{ }$ & $\sqrt{|c|}$ Trade Off \\
\hline $\begin{array}{c}\text { Basic PLL with } \\
\text { integer division }\end{array}$ & $\sqrt{ }$ & $\sqrt{ }$ & $\sqrt{ }$ & $\sqrt{ }$ & $\sqrt{ }$ \\
\hline $\begin{array}{c}\text { Multiloop PLL } \\
\text { Difficult }\end{array}$ & $\sqrt{ }$ & $\sqrt{ }$ & $\sqrt{ }$ & $\sqrt{ }$ \\
\hline $\begin{array}{c}\text { Fractional-Division } \\
\text { Loop }\end{array}$ & $\sqrt{ }$ & $\sqrt{ }$ & $\sqrt{ }$ & & $\sqrt{ }$ \\
\hline
\end{tabular}

Table 2.2 Comparison of frequency synthesis techniques.

\subsubsection{The issue of monolithic implementation}

The development of monolithic frequency synthesizer circuits for high-frequency applications such as timing recorery circuits, radio and UHF receivers, cordless telephones, and mobile radio has been under way for more than iu years. Much of the recent work on frequency synthesizers has used high-speed compound semiconductor or 
silicon bipolar IC technology to demonstrate operation at submicrowave or microwave bands. Figure 2.7 shows a typical approach for a high-resolution high-frequency PLL synthesizer.

\subsubsection{Technology limitations}

PLL components have been traditionally implemented using different technologies as illustrated in Figure 2.7. Most low-frequency digital circuits utilize low-power highdensity CMOS processes. Although some implementations have been reported to exhibit operating frequencies up to a few gigahertz, there has been no report to date on the use of a CMOS VCO operating in a gigahertz range. These conflicting mixed high and low frequency technologies inhibit the realization of a complete circuit integration.

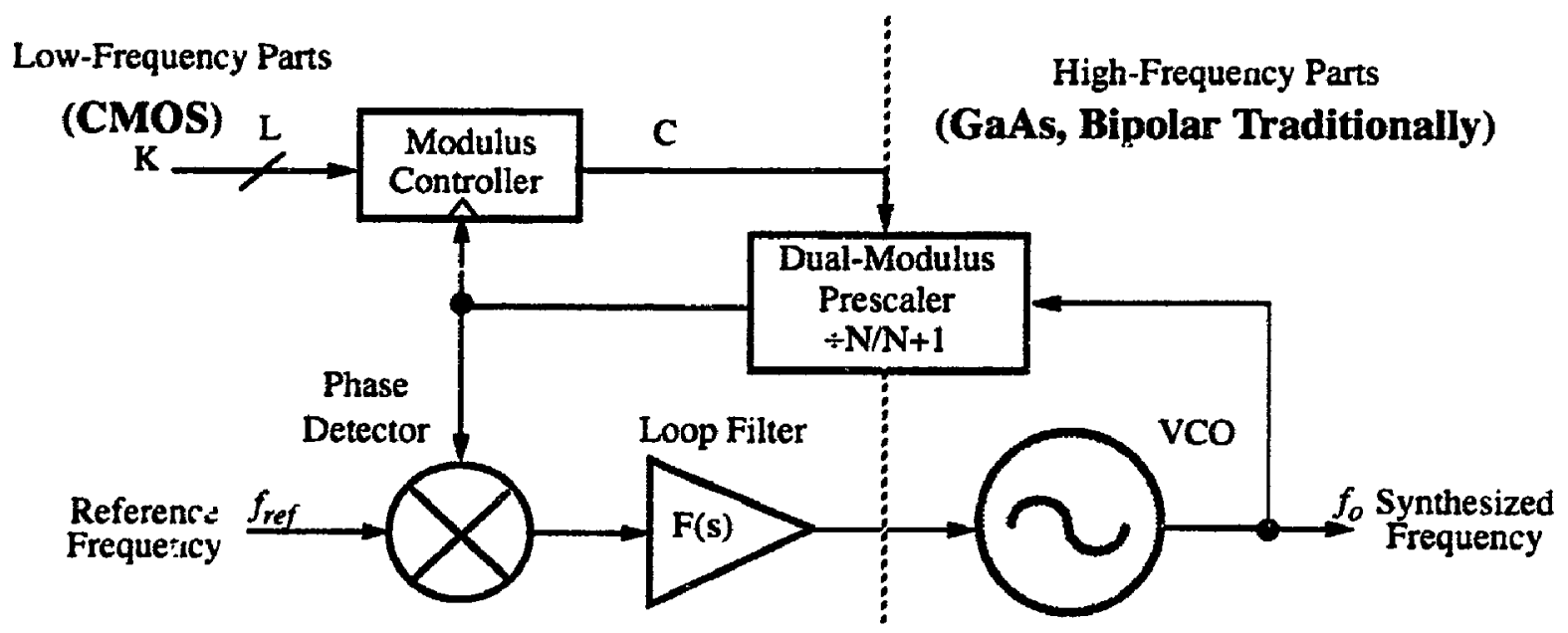

Figure 2.7 Typical building blocks for a modern RF PLL frequency synthesizer.

To evaluate the operation and feasibility of a fully integrated PLL circuit working for high frequency applications, it may be helpful to relate this realization to the state-ofthe-art design specifications issued in the past five years. The following tables summarize the performance information of reported implementations of bipolar, CMOS and GaAs PLL implementation technologies. 


\begin{tabular}{|c|c|c|c|c|c|c|c|}
\hline References & [3]'89 & {$[18]^{\prime} 91$} & {$[19]^{9} 91$} & {$[20]^{\prime} 92$} & {$[21]^{\prime 92}$} & {$[22]^{\prime 92}$} & {$[23]^{9} 93$} \\
\hline Technology & $2 \mu \mathrm{m}$ & $10 \mathrm{GHz} \mathrm{f}_{\mathrm{T}}$ & $10 \mathrm{GHz} \mathrm{f}_{\mathrm{T}}$ & NA & $10 \mathrm{GHz} \mathrm{f}_{\mathrm{T}}$ & $25 \mathrm{GHz} \mathrm{f}_{\mathrm{T}}$ & $30 \mathrm{GHz} \mathrm{f}_{\mathrm{T}}$ \\
\hline Applications & Universal & $\begin{array}{l}\text { PLL. } \\
\text { Clock Gen. }\end{array}$ & $\begin{array}{l}\text { PLL, } \\
\text { Clock Gen. }\end{array}$ & $\begin{array}{l}\text { PLL, } \\
\text { Clock Gen. }\end{array}$ & $\begin{array}{c}\text { VHF/UHF } \\
\text { Tuners }\end{array}$ & $\begin{array}{l}\text { PLL, } \\
\text { Clock Gen }\end{array}$ & $\begin{array}{l}\text { PLL. } \\
\text { Clock Gen }\end{array}$ \\
\hline $\begin{array}{l}\text { Usable Freq. } \\
\text { (MHz) }\end{array}$ & 350 & 622 & $52-155$ & 155 & VHF $-1,000$ & $600-1,500$ & 2,300 \\
\hline $\begin{array}{l}\text { VCO Range } \\
(\mathrm{MHz})\end{array}$ & \pm 180 & $497-746$ & - & - & . & $500 \cdot 1,600$ & - \\
\hline $\begin{array}{l}\text { Supply } \\
(\mathrm{mW})\end{array}$ & $\begin{array}{c}5 \mathrm{~V} \\
270 \mathrm{~mW}\end{array}$ & $\begin{array}{c}5 \mathrm{~V} \\
1250 \mathrm{~mW}\end{array}$ & $\begin{array}{c}5 \mathrm{~V} \\
575 \mathrm{mw}\end{array}$ & $\begin{array}{l}-5.2 \mathrm{~V} \\
350 \mathrm{~mW}\end{array}$ & $\underset{200 \mathrm{~mW}}{\mathrm{SV}}$ & $\begin{array}{c}-4.5 \mathrm{~V} \\
2000 \mathrm{~mW}\end{array}$ & $\begin{array}{c}-5 \mathrm{~V} \\
100 \mathrm{~mW}\end{array}$ \\
\hline $\begin{array}{c}\text { Lock in/Jitter } \\
\text { (sec.) }\end{array}$ & 57ps./NA & $-114 \mathrm{ps}$. & $-/ 153 \mathrm{ps}$. & $-/ 14 p s$. & $\%$ & $-i 19 p s$. & $-/ 1650 \mathrm{ppm}$ \\
\hline$\underset{\left(\mathrm{mm}^{2}\right)}{\operatorname{Areg}}$ & 0.50 & 7.84 & 15.03 & 10.75 & 12.00 & 12.25 & - \\
\hline Remarks & $\begin{array}{l}\text { Off-chip } \\
\text { Inductor. } \\
\text { Loop filter }\end{array}$ & $\begin{array}{l}\text { Ring OSC. } \\
\text { vCo }\end{array}$ & $\begin{array}{l}\text { Emitter-cou- } \\
\text { pled multivi- } \\
\text { brator VCO. }\end{array}$ & $\begin{array}{c}\text { Ring OSC. } \\
\text { VCo }\end{array}$ & $\begin{array}{l}\text { Off-chip } \\
\text { Resonator, } \\
\text { Loop filter }\end{array}$ & $\begin{array}{c}\text { Ring OSC. } \\
\text { VCo }\end{array}$ & $\begin{array}{l}\text { Enutler-cou- } \\
\text { pled mulivi- } \\
\text { brator VCO. }\end{array}$ \\
\hline
\end{tabular}

Table 2.3 Bipolar monolithic PLLs.

Observe that even the most highly-integrated commercially available PLL circuits often require some external components, specifically, the loop filter and the VCO. Because the long time constants usually required by a continuous-time loop filter cannot be easily realized with integrated components, off-chip RC networks are usually implemented for filtering.

In the case of VCOs, a review of the literature shows that including VCO circuits on the same die with other digital circuitry results in undesirable noise and extraneous signal interference [14]. Still, some recently appearing PLLs show the potential to integrate the loop filter and VCO circuits without the use of external components. Of course, such designs compromise the overall performance. Nevertheless, the benefits of a single-chip solution makes them so attractive that there have been a numerous attempts to develop high-frequency single chip PLLs. 


\begin{tabular}{|c|c|c|c|c|c|c|}
\hline \multirow{2}{*}{ References } & \multicolumn{4}{|c|}{ BiCMOS } & \multicolumn{2}{|c|}{ GaAs } \\
\hline & {$[24]^{\prime} 87$} & [25]'91 & {$[26]^{\prime} 92$} & {$[27]^{\prime 93}$} & {$[28]^{\prime} 91$} & {$[29]^{\prime} 92$} \\
\hline Technology & $2 \mu m$ & I $\mu \mathrm{m}, 9 \mathrm{GHz} \mathrm{f}_{\mathrm{T}}$ & $\mathrm{I} \mu \mathrm{m}$ & $1.2 \mu \mathrm{m}$ & $0.9 \mu \mathrm{m}$ AT\&T & $0.4 \mu \mathrm{m}$ \\
\hline Applications & $\begin{array}{c}\text { Timing Recov- } \\
\text { ery }\end{array}$ & Clock Gen. & Ponable Phone & $\begin{array}{c}\text { Data Transceiv- } \\
\text { ers }\end{array}$ & Clock Gen. & $\begin{array}{c}\text { DDS embedded } \\
\text { PLLS }\end{array}$ \\
\hline $\begin{array}{l}\text { Usable Freq. } \\
\text { (MHz) }\end{array}$ & $1 \cdot 128$ & 3.90 & $600-1200$ & $100-315$ & 2500 & $1000-2000$ \\
\hline $\begin{array}{c}\text { VCO Range } \\
\text { (MHz) }\end{array}$ & $\begin{array}{c}200 \\
(\text { Max. })\end{array}$ & $\begin{array}{c}100 \\
(\operatorname{Max} .)\end{array}$ & $\begin{array}{c}1200 \\
\text { (Max.) }\end{array}$ & $\begin{array}{c}315 \\
\text { (Max.) }\end{array}$ & 4000 & NA \\
\hline $\begin{array}{l}\text { Supply } \\
\text { (mW) }\end{array}$ & $\begin{array}{c}5 \mathrm{v} \\
160 \mathrm{~mW}\end{array}$ & NA & $\begin{array}{c}2.7-5.5 \mathrm{~V} \\
38 \mathrm{~mW}\end{array}$ & $\begin{array}{c}5 \mathrm{~V} \\
1.000 \mathrm{mw}\end{array}$ & $\underset{1250 \mathrm{~mW}}{5.2 V}$ & $\frac{+5 \mathrm{~V}}{1300 \mathrm{mw}}$ \\
\hline $\begin{array}{c}\text { Lock In/SItter } \\
\text { (sec.) }\end{array}$ & NAJNA & $11 \mu \mathrm{s} / \mathrm{NA}$. & NA/NA & $\%$ & $700 \mathrm{~ns}$ /NA & NAVNA \\
\hline $\begin{array}{c}\text { Areq } \\
\left(\mathbf{m m}^{2}\right)\end{array}$ & 6.09 & 0.31 & 6.19 & 18 & NA & 486,347 \\
\hline Remarks & $\begin{array}{l}\text { off-chip Loop } \\
\text { filter }\end{array}$ & $\begin{array}{l}\text { Gate Length } \\
\text { pmos }-1.1 \mu \mathrm{m} \\
\text { nmos }-0.9 \mu \mathrm{m}\end{array}$ & $\begin{array}{l}\text { VCO and loop } \\
\text { filter off-chip }\end{array}$ & $\begin{array}{l}\text { VCO and loop } \\
\text { filter on-chip }\end{array}$ & $\begin{array}{l}\text { Silicon-Bipolar } \\
\text { off-chip loop } \\
\text { control }\end{array}$ & $\begin{array}{c}\text { Only Divider } \\
\text { and } \mathbf{S} / \mathrm{H} \text { circuits }\end{array}$ \\
\hline
\end{tabular}

Table 2.4 BiCMOS and GaAs monolithic PLLs.

Recently the trend has reportedly been to use BiCMOS technologies more widely. Obviously this can be shown to have advantages in the use of both the high-speed bipolar and power conserving CMOS circuitry. However, experience has shown that the use of high-frequency parts has been limited to the programmable frequency divider, phase detector and loop filter with off-chip RC filtering $[25,26]$. Some researchers have integrated VCOs on chip with internal and external LC tank circuits, though none has shown the implementation of a VCO without an inductor or resonant circuit.

There has also been some interest in GaAs implementation of PLL circuits in recent years (see Table 2.4). Many of the recent papers report the use of GaAs to enhance the speed of components in fractional- $\mathbf{N}$ frequency synthesizers such as prescaler and VCOs $[29,30]$. Although GaAs devices operate at high frequency, Table 2.4 shows that GaAs PLLs' disadvantages could outweigh their performance. The contributing factors are high 
circuit complexity, low density of integration, substantial power consumption, and high cost. As a result, monolithic GaAs PLLs are not commercially preferred for application in mobile radios.

\begin{tabular}{|c|c|c|c|c|c|c|c|c|}
\hline References & [31]'85 & {$[32]^{\prime 92}$} & {$[33]^{\prime 92}$} & {$[34]^{\prime} 92$} & {$[35]^{\prime 92}$} & {$[36]^{\prime 93}$} & {$[37]^{\circ 93}$} & {$[38]^{\prime} 93$} \\
\hline Technology & $\begin{array}{l}1.8 \mu \mathrm{m} \\
\text { NMOS }\end{array}$ & $\begin{array}{l}1.0 \mu \mathrm{m} \\
\text { NMOS }\end{array}$ & $\begin{array}{l}1.0 \mu \mathrm{m} \\
\mathrm{CMOS}\end{array}$ & $\begin{array}{l}0.9 \mu \mathrm{m} \\
\mathrm{CMOS}\end{array}$ & $\begin{array}{l}\text { 0.8um } \\
\text { CMOS }\end{array}$ & $\begin{array}{l}0.9 \mu \mathrm{m} \\
\text { CMOS }\end{array}$ & $\begin{array}{l}0.7 \mu \mathrm{m} \\
\text { CMOS }\end{array}$ & 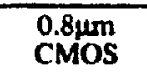 \\
\hline Applications & Universal & $\begin{array}{c}\text { PLL, } \\
\text { Clock Gen. }\end{array}$ & $\begin{array}{l}\text { PLL, } \\
\text { Clock Gen. }\end{array}$ & Universal & $\begin{array}{l}\text { uP-Clock } \\
\text { Gen. }\end{array}$ & $\begin{array}{l}\text { PLL. } \\
\text { Clock Gen. }\end{array}$ & $\begin{array}{l}\text { PLL. } \\
\text { Clock Gen. }\end{array}$ & $\begin{array}{c}\text { PLL. } \\
\text { Clock Gien. }\end{array}$ \\
\hline $\begin{array}{l}\text { Usable Freq. } \\
\text { (MHz) }\end{array}$ & $40 \cdot 1200$ & 1000 & $30-128$ & $5 \cdot 180$ & $5-110$ & 660 & 266 & 3.30 \\
\hline $\begin{array}{c}\text { VCO Range } \\
(\mathrm{MHz})\end{array}$ & NA & NA & NA & NA & $10 \cdot 220$ & $450-690$ & NA & $50-3.30$ \\
\hline $\begin{array}{l}\text { Supply } \\
\text { (mW) }\end{array}$ & $\begin{array}{l}3.3-5 \mathrm{~V} \\
125 \mathrm{~mW}\end{array}$ & $5 \mathrm{~V}, 550 \mathrm{~mW}$ & $5 \mathrm{~V}, 75 \mathrm{~mW}$ & $5 \mathrm{~V}, 250 \mathrm{~mW}$ & $5 \mathrm{~V}, 16 \mathrm{~mW}$ & $\begin{array}{l}3.25 .5 \mathrm{~V} \\
600 \mathrm{~mW}\end{array}$ & $5 \mathrm{~V}, 800 \mathrm{~mW}$ & $5 \mathrm{~V}, 800 \mathrm{~mW}$ \\
\hline $\begin{array}{l}\text { Lock in/Jitter } \\
\text { (sec.) }\end{array}$ & NANA & NA/13ps. & NA/50ps. & 5ms./56.ps. & 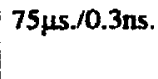 & NA/NA & NA/200ps & NA/NA \\
\hline $\begin{array}{c}\text { Areg } \\
\left(\mathrm{mm}^{2}\right)\end{array}$ & 1.57 & 1.68 & 1.35 & 8.90 & 0.31 & 0.3 & 7.2 & 1.25 \\
\hline Remarks & $\begin{array}{l}\text { Without } \\
\text { vco }\end{array}$ & Ring OSC. & Ring OSC. & Ring OSC. & Ring OSC. & Ring OSC. & Ring OSC. & Ring OSC. \\
\hline
\end{tabular}

Table 2.5 MOS monolithic PLLs.

The widely used, low-cost CMOS process has so far remained the most popular in PLL circuits operating up to a few hundred megahertz, as shown in results partially summarized in Table 2.5. Evidently, the availability of the submicron CMOS process has shown that the speed of CMOS PLLs has significantly increased compared to that of PLLs in the past. Note, however, that the maximum operating frequencies of NMOS PLLs are much higher than CMOS PLLs. The challenge is then to develop CMOS analog circuits for high frequency applications. 


\subsubsection{Frequency synthesizer implementation technology}

As reported in the panel discussion held at the CICC'93 conference, "Personal Communications in the Next Decade" [39], all IC industries involved in this field have shown aggressive progress in the development of monolithic CMOS personal communication terminals. For example, a 700-MHz low-noise amplifier (LNA) and a 900-MHz mixer implemented in special 1.2 to $1.5 \mu \mathrm{M}$ CMOS processes $[40,41]$ have achieved superior or comparable performance to those implemented in silicon bipolar technology. However, the limiting factor in the realization of a single-chip CMOS synthesizer within the existing technologies is the availability of a gigahertz frequency range CMOS VCO. If high-speed CMOS VCO capabilities become available, system integration for radio components on a single chip would be possible, resulting in low cost, low power and high reliability, all of which are important to the future deployment of global wireless communications.

\subsection{Summary}

Following a review of viable frequency synthesis techniques, the fractional- $N$ phase-locked loop has been selected as the most suitable synthesis technique for use in wireless communications. It is superior in high frequency resolution, switching speed and ease of monolithic implementation. As mentioned previously, MOS and silicon bipolar technologies remain the most favorable processes in phase-locked loop circuit designs for mid to high frequency applications. Based on the research previously presented, it can be seen that the operating frequency of PLLs has increased considerably over the past five years. These improvements prove that not only should suitable circuit configurations be employed in the high-speed circuit designs, but so should the technologies which compliment their operation. High-speed fully integrated PLLs can now be achieved at speeds above a gigahertz. This has become possible through advancements in current IC processing. It should be noted, however, that the performance of CMOS PLLs has not met 
the requirements for mobile communications. Only bipolar integrated circuits are capable of serving those requirements. Yet, the relatively high power consumption of bipolar PLLs has pushed many researchers to look for other alternatives, such as the possibility of highspeed MOS circuits. Since MOS processes continue to scale down to smaller geometry. this may allow the realization of MOS integrated circuits that operate beyond gigahertz frequencies.

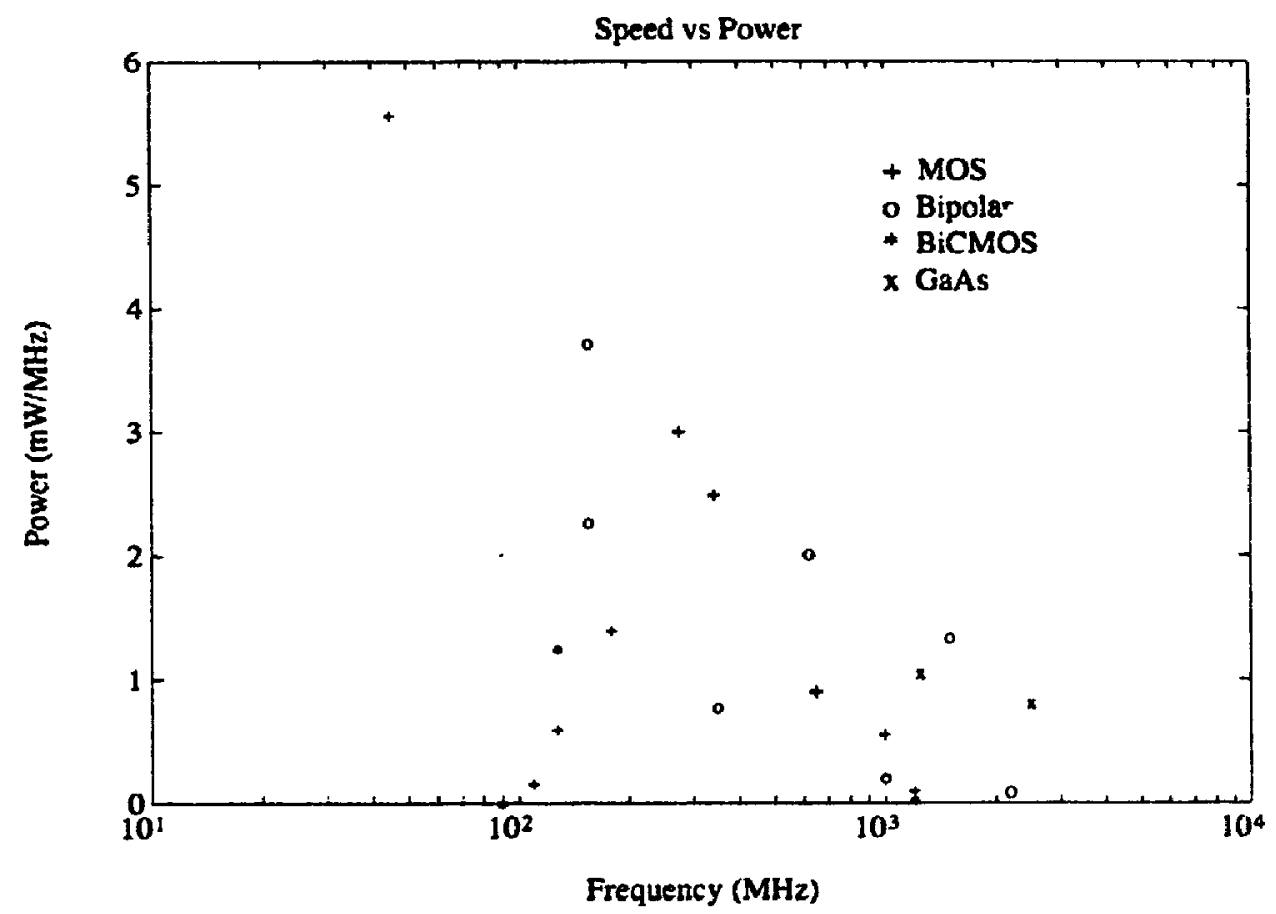

Figure 2.8 Speed and power comparison graph of different PLL technologies.

It was also mentioned earlier that some PLL components might be preferred in implementations using CMOS processes due to the benefits of their high level of integration. Therefore, it is worthwhile to realize other loop components of the PLLs with CMOS technology. The next chapter discusses the issues of design and analysis for CMOS PLL components which could be realized as building blocks for a monolithic mobile trarsceiver. 


\section{Chapter 3}

\section{The Realization of High-Speed PLL Components in CMOS Process}

As introduced in Chapter 2, the design of a frequency synthesizer for a mobile radio transceiver can best be implemented with fractional-division phase-locked loops (PLL). The selected implementation technology for the phase-locked loop components is CMOS, which supports the demand for smaller, low-power terminals that provide reasonable noise performance at lower cost. In order to build a monolithic CMOS frequency synthesizer with performance comparable to that of a multi-chip structure, proper PLL components must be selected. Otherwise the noise contribution of individual digital components can dominate the total system noise.

This chapter describes the design considerations associated with the PLL system as well as its components. The focus is on the voltage controlled oscillator (VCO) since it has a direct impact on the overall performance of the synthesizer. High-speed and low-noise CMOS VCO structures are investigated and proposed. The remaining components, such as phase detector, loup filter and frequency divider, are described briefly. 


\subsection{PLL synthesizer components}

The basic diagram of a high-resclution fractional-N PLL is again shown in Figure 3.1. In lock condition, the VCO generates a fixed frequency $f_{o}$. If the tuning word changes, the VCO generates a new synchronized frequency corresponding to the feedback action of the PLL.

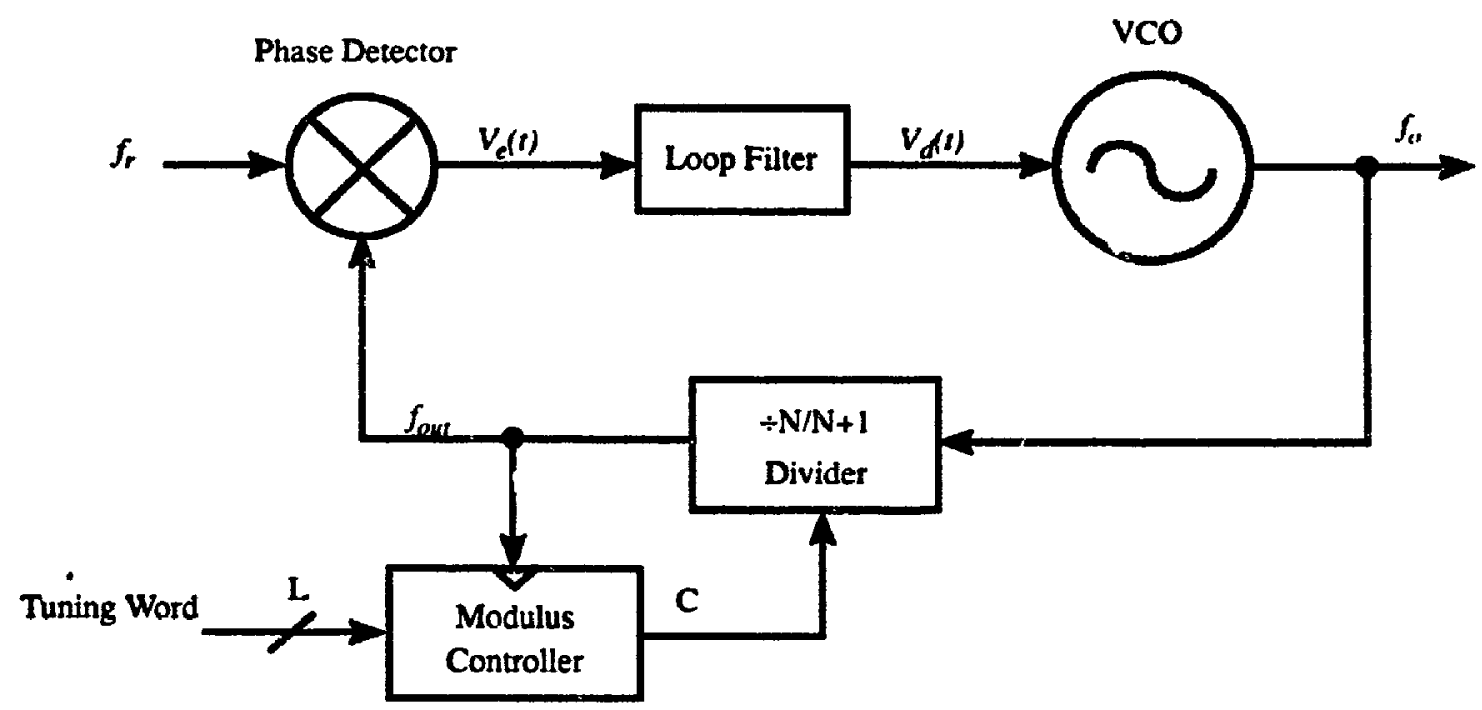

Figure 3.1 A VCO as a component of a fractional division PLL.

\subsubsection{Design issues and considerations}

The key issues associated with the design of a PLL synthesizer are power consumption and in-band and out-of-band signal quality. Typical carrier frequencies for mobile radio applizations range from a few hundred megahertz to several gigahertz. The synthesizer is powered up continuously or in burst mode in order to transmit and receive communication channels. Thus, the circuits in the PLL are required to consume the lowest possible power in order to prolong battery life. 
If CMOS is selected to support this requirement, the problem of integrating all the PLL building blocks into a single silicon chip is made more difficult, since a PLL synthesizer consists of both analog and digital components. Noise and interference are often encountered for such a monolithic combination. Another limiting factor for CMOS circuits is their maximum operating frequency. CMOS is often reported to be too slow for radio circuits. To successfully integrate all the PLL building blocks into a single silicon chip, the problem associated with noise and speed must be resolved.

Bearing these design problems and trade-offs in mind, designers must carefully select suitable design techniques and circuit ideas that help to overcome and possibly complement the trade-off issues presently encountered.

\subsection{VCO design}

Since the quality of the PLL output is almost entirely determined by the VCO performance, attention must be paid to the VCO specifications.

\subsubsection{Key parameters for VCO}

General requirements for a high-quality VCO include high spectral purity, linear voltage-to-frequency transfer characteristic and good frequency stability to power supply and temperature variation. However, for mobile radio applications, the VCO must also comply with the basic requirement of low power consumption and cost of fabrication as well.

While high linearity and wide tuning range may be necessary in some applications, in wireless communications where bandwidths are limited to a few tens of megahertz, tuning range and linearity are of little concern (see Table 2.1). Long term frequency stability as a function of temperature is not a problem if the rate of change of frequency drift is within the PLL loop bandwidth [42]. The feedback action of the PLL suppresses the frequency error with a magnitude that is a direct function of the open loop gain. The significant VCO properties that must be considered remain as follows. 
- Spectral purity

- Speed and power consumption

- Monolithic suitability

\subsubsection{High spectral purity}

An oscillator's spectral purity is a direct measure of its phase noise which indicates the phase stability or short term stability of a particular oscillator configuration. For a VCO to be suitable for a mobile radio PLL application, it has to meet the maximum spurious requirement set by a given transmission standard. For example, the Electronic Industries Association (EIA) standard for a $30-\mathrm{kHz}$ channel spacing cellular telephone [43] requires that phase noise present in the synthesized output be at least $-60 \mathrm{dBc} / \mathrm{Hz}$ at $45 \mathrm{kHz}$ offset from the carrier and greater than $-75 \mathrm{dBc} / \mathrm{Hz}$ above $45-\mathrm{kHz}$ offset from the carrier.

In a PLL, VCO phase noise components within the kop bandwidth can be greatly suppressed by the loop filter correction. However, at large offset frequencies which lie outside the loop bandwidth, phase noise of the PLL is largely the component introduced by its orrillator. Thus, it is essential that the oscillator's phase noise be kept as low as possible.

\subsubsection{Frequency of operation and power consumption}

The ma:imum synthesized frequency of the PLL is inevitably limited by the maximum frequency of the VCO. Therefore, with a given communication standard, using CT2 as an example, the maximum output frequency is required to exceed $894 \mathrm{MHz}$ (see Table 2.1).

As portable terminals are battery operated the requirement for low power consumption directly translates into lower operating voltages and fewer components operating at high speeds which become the key specifications for frequency synthesizer design. 


\subsubsection{Suitability for circuit integration}

The VCO configurations best suited to monolithic design have become more popular as advances in VLSI have become more accessible. A single-chip design with no external components certainly reduces manufacturing costs and the size of the terminals. However, one crucial property of such a monolithic VCO design is the VCO topology which can be incorporated with other digital circuitry without excessive noise deterioration of the VCO spectral output. Hence, VCO structures that can be both part of highly integrated circuits and work in a $\mathrm{GHz}$ range on silicon pose a major design challenge.

\subsubsection{Monolithic VCO design}

A monolithic oscillator can be realized using a tuned oscillator whose frequency is determined by reactive elements or by a relaxation oscillator, also - alled a multivibrator, which operates by altenately changing and discharging an energy storage element (usually a capacitor).

\subsubsection{LC tank or tuned oscillators}

The frequency selective feedback of the resonant oscillator can be implemented using a simple LC circuit such as the one shown in Figure 3.2 [44] or resonators such as a SAW or a crystal resonator. Owing to the tuned nature of the resonant circuits, these oscillators are less influenced by temperature, noise, and supply voltage. However, they are all basically incompatible with monolithic IC technology. LC tank, SAW and crystal resonators all remain external to the silicon chip in order to meet the requisite spectral purity $(Q)$, and often cost more than the circuit itself.

In semi-integrated circuit form, resonant oscillators often employ off-chip LC tank circuits due to the difficulty in controlling process variations which can lead to long term frequency drift. Although there is current research in the form of silicon bipolar on-chip LC VCO architectures, the achievable value of $Q$ remains low (about 5) [45]. In addition, the inductors built cn silicon occupy a substantially large area compared to other on-chip VCO designs. 


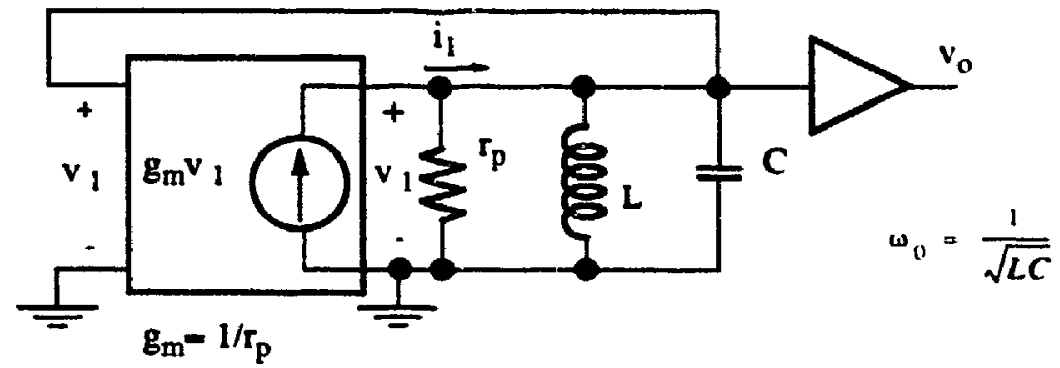

Figure 3.2 Basic resonant circuit.

\subsubsection{Multivibrator or relaxation based oscillators}

Multivibrators or so-called relaxation oscillators can be emitter coupled oscillators (ECO) as shown in Figure 3.3.a, or ring oscillators (Figure 3.3.b). Both circuits operate by alternatively charging and discharging a timing capacitor between two initially set threshold voltage levels. Since relaxation oscillators do not require any external components for oscillation, they are now becoming preferable in most high frequency fully integrated PLL circuits.

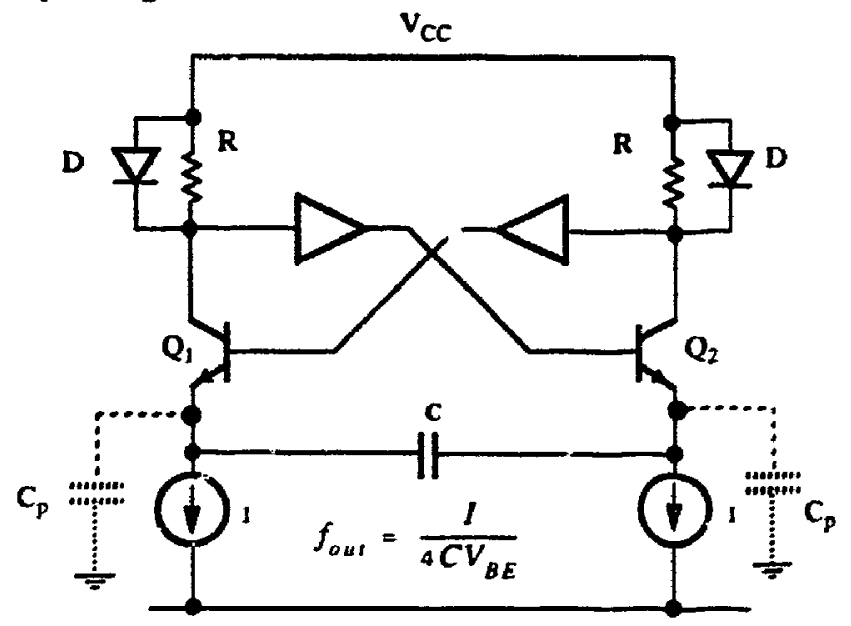

a) Relaxation Oscillator (Emitter-Coupled Multivibrator)

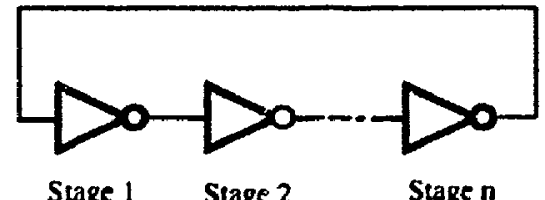

Stage

Fıgure 3.3 Multivibrator oscillators (a) Emitter coupled multivibrator; (b) Ring oscillator. 
The bandwidth of the feedback circuits of multivibrators is much wider than those of the tuned oscillators, resulting in higher phase noise output. Consequently, the free running spectral purity of relaxation oscillators is inferior. The PLL architecture must have some form of compensation, or use some special techniques, to accommodate the wide tuning range $[42,44]$.

Ring oscillators, which are good candidates in terms of speed, consist of a chain of logic gates in a closed loop configuration. The oscillating frequency is determined by the total propagation delay around the loop. Control of the oscillating frequency can be gained by changing the voltage or current which controls the time delay in each inverting stage. Although ring oscillators are characterized by higher phase noise than ECOs, almost all monolithic PLLs that have been reported recently employ the ring oscillator principle.

\subsubsection{Comparison of bipolar ECOs and MOS relaxation oscillators}

An inductorless oscillator based on an emitter coupled multivibrator structure or ring oscillator topology, as shown in Figure 3.3, can be modelled as a basic relaxation or Schmitt-trigger oscillator, as illustrated in Figure 3.4 [42].

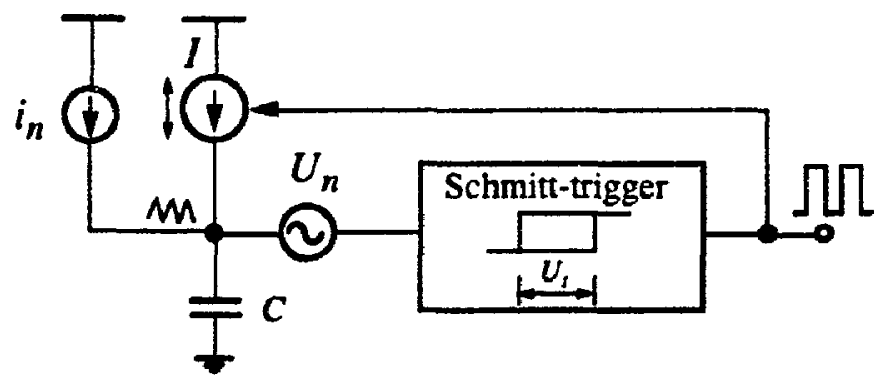

Figure 3.4 Generalized circuit for relaxation oscillator noise analysis.

Sources of phase noise in this model are a toise current $i_{n}$ parallel with current source $I$, and a noise voltage $U_{n}$ in series with the timing capacitor. The frequency of oscillation, where $U_{t}$ is the threshold voltage difference, is given by 


$$
f_{o}=\frac{l}{2 U_{1} C}
$$

Modulation of the oscillation frequency can occur at low and high frequencies depending on the frequency of noise sources $f_{m}$. Different methods must be employed to analyze the phase noise. If the noise frequency is much lower than the oscillation frequency $f_{o}$, there is only normal frequency modulation of the oscillator. For higher noise frequencies, however, the effects of the switching action become significant through aliasing, which in turn modulates the frequency of oscillation.

An analysis by Verhoeven [42] showed that for the low-frequency noise, there are two noise sources that contribute to oscillator modulation, both the noise current $i_{n}$ and the noise voltage $U_{n}$. The single-sided spectral phase noise density $\mathcal{L}\left(f_{m}\right)[\mathrm{dBc} / \mathrm{Hz}]$ contributed by $i_{n}$ and $U_{n}$ can be expressed by Eqns. (3.2) and (3.3) respectively.

$$
\begin{gathered}
\mathcal{L}_{i}\left(f_{m}\right)=\frac{2 k T}{I^{2} R_{i}}\left(\frac{f_{o}}{f_{m}}\right)^{2} \\
\mathcal{L}_{U}\left(f_{m}\right)=\frac{2 k T R_{u}}{U_{t}^{2}}\left(\frac{f_{o}}{f_{m}}\right)^{2}
\end{gathered}
$$

where $R_{i}$ and $R_{u}$, in the case of an emitter coupled multivibrator, are the equivalent thermal noise resistance of the current source $I$ and the noise sources in series with $C$ respectively. The product of $I^{2} R_{i}$ indicates the power consumption of the oscillator. In order to achieve a good phase noise performance, a certain minimum power consumption in the current sources is unavoidable.

For high-frequency noise, the phase noise caused by the switching current noise $i_{n}$ is negligible because of the integrating action of the timing capacitor. Therefore, the dominant noise source is $U_{n}$ and is given by 


$$
\begin{aligned}
& \qquad \mathcal{L}\left(f_{m}\right)=\alpha \frac{16 k T R_{u}}{U_{i}^{2}}\left(\frac{f_{o}}{f_{m}}\right)^{2} \\
& \text { where } \alpha=\frac{B_{\text {conv }}}{2 f_{o}}
\end{aligned}
$$

The term a denotes the number of aliased switching spectra and is a function of the noise bandwidth $B_{\text {conv }}$ and $f_{o}$ (see detailed analysis in [42]). It is apparent from Eq. 3.2 to 3.4 that lower phase noise ECOs are only achieved with higher current. With increased current $I$, and the same oscillation frequency, timing capacitance $C$ must also increase.

The architecture of a high-frequency and low-noise ECO does not accommodate a low-power design because it requires considerable design compromises between noise, power dissipation and maximum frequency of operation.

Next, a relaxation ring oscillator based on the construction of all MOS devices as opposed to an ECO structure will be analyzed. Using the same oscillation mechanism as depicted in Figure 3.4, the voltage comparator, as modelled by a Schmitt trigger, is an inverter cell as shown in Figure 3.5.

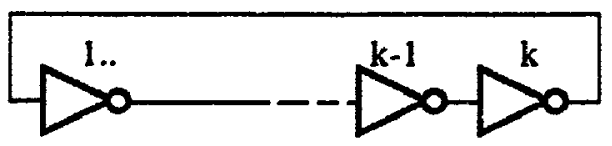

(a) Ring oscillator

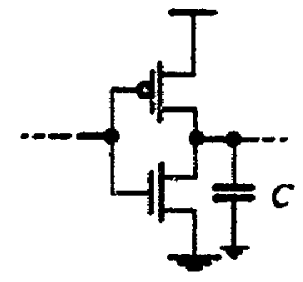

(b) CMOS inverter with its parasitic

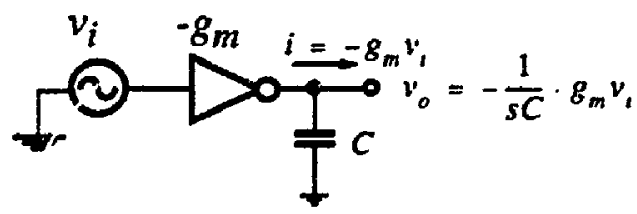

(c) Equivalent circuit for an integrator

Figure 3.5 Ring oscillator based on MOS inverter ring structure. 
From Eq. 3.1, the period of the oscillation $T_{o}$ is

$$
T_{0}=\frac{2 U_{t} C}{I}
$$

Assuming at the threshold crossing where the current threshold can be expressed as $I_{t h}=g_{m} \cdot v_{i}$, then the random fluctuation of $U_{t}$ induces corresponding fluctuations in $T_{\theta}$ resulting in

$$
d t=\frac{2 C}{I_{l h}}\left(d V_{t h}\right)
$$

where $V_{t h}$ is the threshold voltage of the inverter. Since both $C$ and $I_{t h}$ are functions of the device's geometries, $C$ can be represented by $C=K_{1} \cdot W \times L$, while assuming all inverter stages a e identical and have linear operation, $I_{t h}=g_{m} \cdot v_{i}$ or $I_{t h}=K_{2} \cdot \sqrt{W / L}$, thus the phase noise in terms of fluctuations in time $d t$, as a function of threshold voltage variation, may be rewritten as

$$
\begin{aligned}
\frac{d t}{d V_{t h}} & =\frac{2 K_{1} \cdot W \times L}{K_{2} \cdot \sqrt{W / L}} \\
\text { or } \quad \frac{d t}{d V_{t h}} & =K_{3} \cdot W^{1 / 2} \times L^{3 / 2}
\end{aligned}
$$

Equation 3.9, above, implies that the feature sizes of the devices used as gain blocks in the oscillator have significant influence on phase noise or jitter value. The jitter will be directly proportional to MOS device sizing in a parasitic only MOS oscillator. Smaller device sizes will result in higher ossillating frequency and lower intrinsic jitter. This indicates an advantage of MOS relaxation oscillators over bipolar multivibrators, especially for a MOS relaxation oscillator structure that employs circuit parasitics. Parasitic MOS oscillator structures provide higher circuit integration and lower power consumption (through smaller feature size). However, the oscillation frequency is related to device speed, and therefore MOS oscillators have to be optimized for speed through architecture and layout techniques. 


\subsubsection{The phase noise generation mechanism}

The equivalent RC multivibrator based on the Schmitt-trigger circuit of Figure 3.6 [46] explains the functional sections of the circuit and illustrates the prcsence of phase noise in the oscillator.

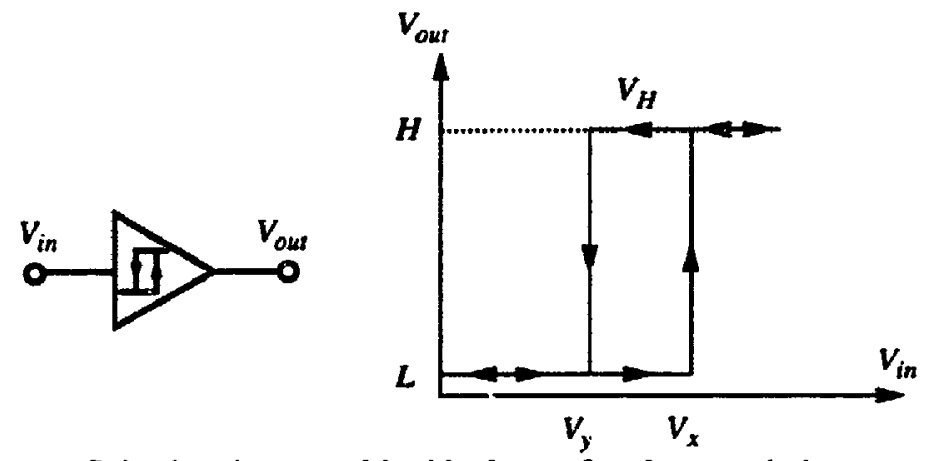

a) Schmitt trigger and its ideal transfer characteristic
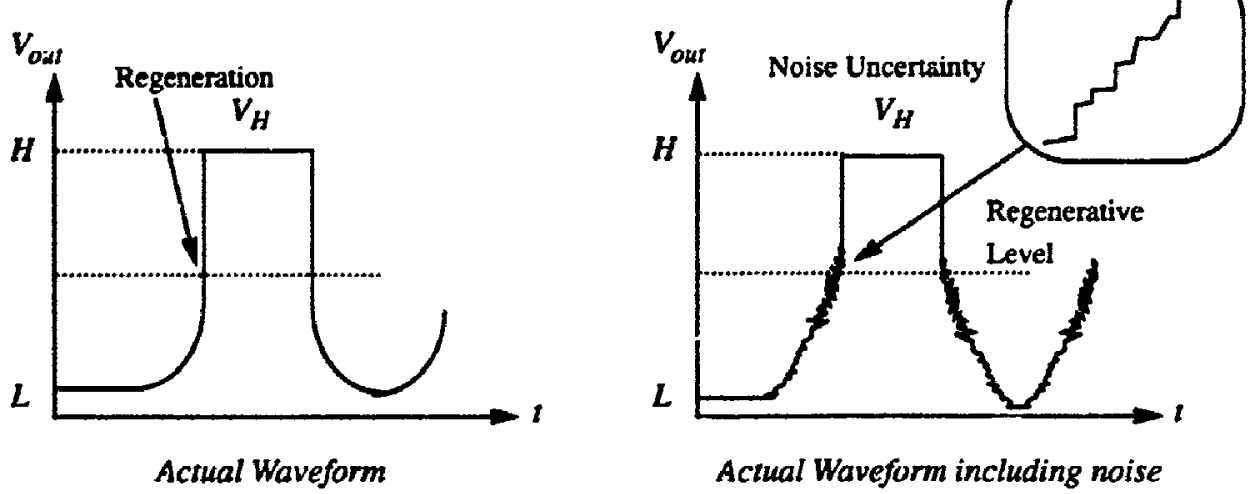

b) Switching waveforms in relaxation oscillator

Figure 3.6 Ideal switching device and the jitter in actual relaxation oscillator.

In the ideal case, the Schmitt trigger is supposed to have a high input impedance, low input bias current and extremely fast switching characteristic, as shown in Figure 3.6.a. In practice, though, the Schmitt trigger has limited switching speed and the typical operating waveform on the input is shown in Figure 3.6.b. The problem arises when noise from the IC substrate and circuit's power supply are induced on the actual input, resulting in an output waveform that has a signal jitter or phase noise component. Onc way of eliminating this jitter is with the use of a very fast switching circuit [46]. 


\subsubsection{Current VCO design}

Table 3.1 provides an insight into the progress of VCO research in the past few years. Note that for applications operating around one gigahertz, which fall in :he frequency range of most cellular applications, Si bipolar off-chip LC and multivibrator VCOs are the most popular configurations.

\begin{tabular}{|c|c|c|c|c|c|c|}
\hline Technology & $\begin{array}{l}\text { Oscillator } \\
\text { Type }\end{array}$ & $\begin{array}{c}f_{\mathbf{T}}(\mathbf{G H z}) \text { or } \\
\text { gate length } \\
(\mu \mathrm{m})\end{array}$ & $\begin{array}{l}\text { Speed } \\
\text { (MHz) }\end{array}$ & $\begin{array}{c}\text { Power } \\
(\mathbf{m w})\end{array}$ & $\begin{array}{l}\text { Phase Noise } \\
\text { at } 100 \mathrm{kHz} \\
\text { offiset (dBC/ }\end{array}$ & Reported by \\
\hline \multirow{5}{*}{$\begin{array}{l}\text { Silicon } \\
\text { Bipolar }\end{array}$} & $\begin{array}{c}\text { Tuned } \\
\text { On-chip LC }\end{array}$ & $10 \mathrm{GHz}$ & 1,800 & 70 & -88 & $\begin{array}{l}\text { Nguyen et al. } \\
|45|^{\prime} 92\end{array}$ \\
\hline & $\begin{array}{l}\text { Tuned } \\
\text { Off-chip LC }\end{array}$ & 10 & 1.100 & 100 & -101 & $\begin{array}{c}\text { Motorola } \\
\text { MC12148 } \\
{[4]^{\prime} 93}\end{array}$ \\
\hline & \multirow[t]{3}{*}{ Multivibrator } & $10 \mathrm{GHz}$ & 1,000 & 65 & - & $\begin{array}{c}W u \\
{[47]^{\prime} 90}\end{array}$ \\
\hline & & $3 \mathrm{GHz}$ & 100 & 261 & .98 & $\begin{array}{c}\text { Verhoeven } \\
{[48\}^{\prime} 92}\end{array}$ \\
\hline & & $3 \mathrm{GHz}$ & 250 & 220 & - & $\begin{array}{l}\text { Lui et al. } \\
\text { [49]'90 }\end{array}$ \\
\hline \multirow[t]{2}{*}{ BiCMOS } & $\begin{array}{c}\text { Tuned } \\
\text { Off-chip LC }\end{array}$ & - & 1,000 & 80 & $\begin{array}{c}-70 \\
\text { (50KHz offset) }\end{array}$ & $\begin{array}{c}\text { Fujitsu MB551 } \\
{[50]^{\prime} 9 !}\end{array}$ \\
\hline & Multivibrator & $2.0 \mu \mathrm{m}$ & 128 & 25 & - & $\begin{array}{l}\text { Kato et al. } \\
{[24]^{\circ} 90}\end{array}$ \\
\hline \multirow[t]{2}{*}{ GaAs } & $\begin{array}{c}\text { Tuned } \\
\text { Ori-chip l.C }\end{array}$ & - & 15,600 & 75 & -85 & $\begin{array}{c}\text { Yamauchi et al. } \\
{[30]^{\prime} 92}\end{array}$ \\
\hline & Multivibrator & - & 17,000 & - & - & $\begin{array}{c}\text { Shigaki et al. } \\
{[51]^{\circ} 92}\end{array}$ \\
\hline \multirow[t]{2}{*}{ NMOS } & \multirow[t]{2}{*}{ Multivibrator } & $1.0 \mu \mathrm{m}$ & 1,400 & 50 & - & $\begin{array}{c}\text { Banu } \\
{[52]^{\prime} 88}\end{array}$ \\
\hline & & $1.0 \mu \mathrm{m}$ & 1,000 & 50 & - & $\begin{array}{c}\text { Abidi } \\
{[32]^{\circ} 92}\end{array}$ \\
\hline \multirow{3}{*}{ CMOS } & \multirow[t]{2}{*}{ Multivibrator } & $1.0 \mu \mathrm{m}$ & 325 & - & - & $\begin{array}{c}\text { Abidi } \\
{[\mathbf{5 3}]^{\prime} 90}\end{array}$ \\
\hline & & $1.2 \mu \mathrm{m}$ & 80 & 20 & - & $\begin{array}{l}\text { Flynn et al. } \\
{[55]^{\prime} 92}\end{array}$ \\
\hline & $\mathbf{N} / \mathbf{A}$ & $1.5 \mu \mathrm{m}$ & 900 & - & $\begin{array}{c}-125 \\
\text { (25 kHz offset) }\end{array}$ & $\begin{array}{c}\text { Camilleri et al. } \\
{[41]^{\prime} 93}\end{array}$ \\
\hline
\end{tabular}

Table 3.1 Collection of VCO implementations to date. 
Power consumption ranges from $50 \mathrm{~mW}$ to $100 \mathrm{~mW}$ and phase noise levels are between $-80 \mathrm{dBc} / \mathrm{Hz}$ to $-101 \mathrm{dBc} / \mathrm{Hz}$ at $100 \mathrm{kHz}$ carrier offset. Presently, no CMOS VCO operating at a gigahertz frequency range has been reported. This comparison indicates that an opportunity exists for new and novel CMOS VCOs designs that can meet the requirements of low power radio applications.

\subsubsection{CMOS oscillator design}

CMOS oscillators can have many advantages over bipolar and NMOS oscillators because they can be built without the need for external components and can result in lower power circuits. As a result, CMOS oscillators have often been used as on-chip timing subcircuits for larger scale monolithic applications.

As reported in several current research papers, circuit techniques have been developed to improve the frequency stability and linearity of inductorless CMOS oscillators. For example, a VCO with improved linearity and jitter improvement was reported by Wakayama [54], while a "MOS VCO with temperature compens:sion was reported by Flynn [55]. These techniques demonstrate that CMOS oscillators are a good prospect for achieving performance comparable to Si bipolar oscillators. Although the use of extemal circuits resulted in improved oscillator characteristics, it was at the expense of increased power consumption and decreased operating speed. Therefore, the speed of the CMOS oscillator has been the limiting factor that restricts its use to only low-io-midfrequency applications. Low power, speed improvement techniques are therefore the major challenges in a CMOS oscillator design.

\subsubsection{Speed limits}

The design of most very high-frequency, low-power consumption on-chip oscillator circuits poses the same challenges with regard to speed. Many nodes in the circuit have an undesirable parasitic capacitance to ground. These nodes not only slow down the 
operation but also cause a parasitic switching current. This implies that high-speed circuits should follow these design principles:

- Minimize all parasitics by using the circuits with minimum complexity

- Search for architectures enabling the circuits to operate at maximum speed

- Utilize unavoidable parasitics; no external reactive elements.

Since speed is of major concern, CMOS oscillators can then be constructed using only the simplest structures, such as a simple odd-number inverter ring oscillator based on an RC relaxation oscillator as illustrated in Figure 3.7.

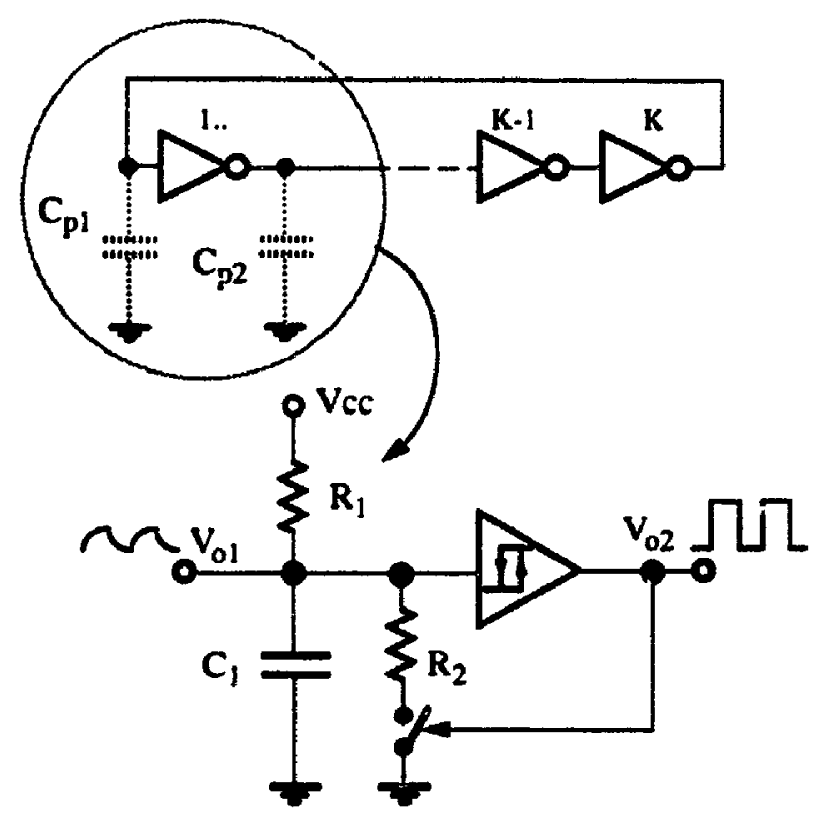

Figure 3.7 Ring oscillator and its representation in RC relaxation configuration.

The inverter ring oscillator has been shown to be the fastest oscillator circuit compared to other oscillator approaches because it is constructed from the lowest complexity circuits. Each inverter in the chain can be represented by a Schmitt trigger and its associated timing components $R_{1}, R_{2}$ and $C_{1}$, that form an $R C$ relaxation oscillator [57]. The inverter ring oscillators that consist of non-physical timing elements $R_{1}, R_{2}$ and $C_{1}$ are therefore the circuit parasitics. Hence, the frequency of oscillation can be extremely high. 
The inverter ring oscillator usually has inherent drawbacks because the frequency output has a high dependence cn temperature and power supply variations. However, when the oscillator is used in a PLL, which is often the case, drift with temperature and power supply is of little concern.

Altematively, a high-speed CMOS oscillator can also be a latching comparator circuit built in the manner of a constant-current charge-discharge oscillator. Figure 3.8 shows the basic current control oscillator (ICO) [57].

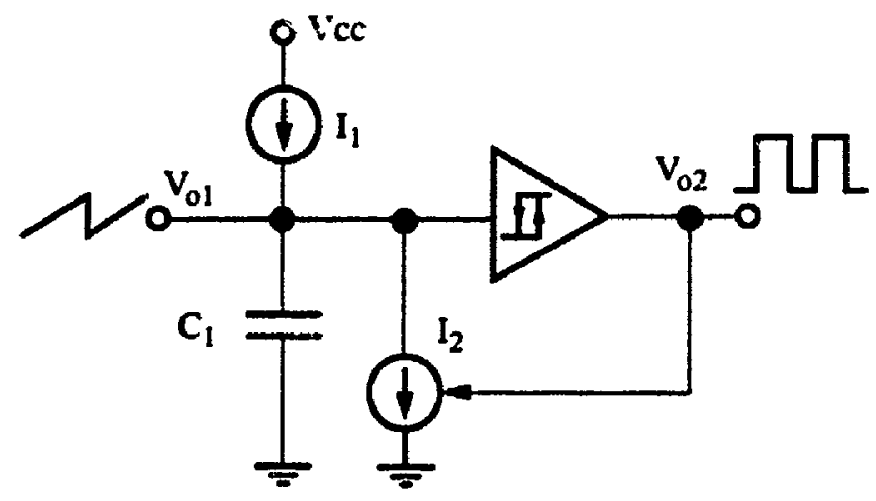

Figure 3.8 Simplified constant-current relaxation oscillator.

The ICO requires a latching regenerative circuit to provide the oscillation. The latch regenerative circuit can be built using a SR latch similar to the one shown in Figure 3.9 [58].

The only reactive element is the capacitor, $\mathrm{C}$, that is charged by the current source $I_{1} \cdot I_{2}$ is used for discharging while $I_{3}$ provides voltage reference levels to the two comparators. In this implementation, the latch develops the memory function, while the capacitor works as an integrator. 


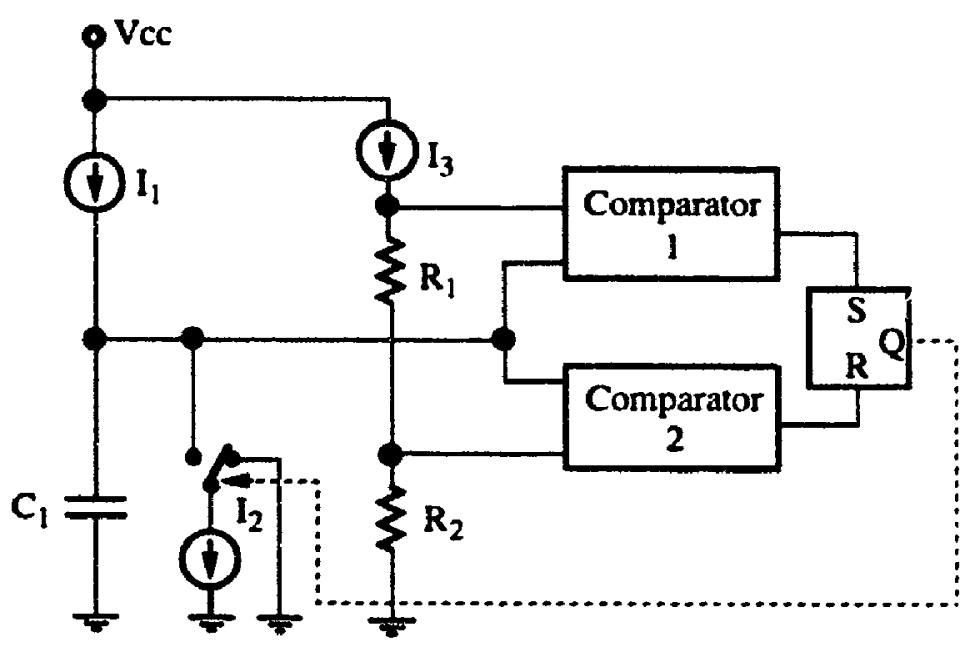

Figure 3.9 Latching circuits for ICO.

The current controlled oscillator as depicted in Figure 3.10 is one of the structures well suited to speed performance. The architecture is based on a known emitter coupled bipolar oscillator. However, the difference here is that the parasitic ground capacitance is utilized, which is suitable for MOS realization. Currently, speeds to a gigahertz range have been achieved with NMOS implementation [52].

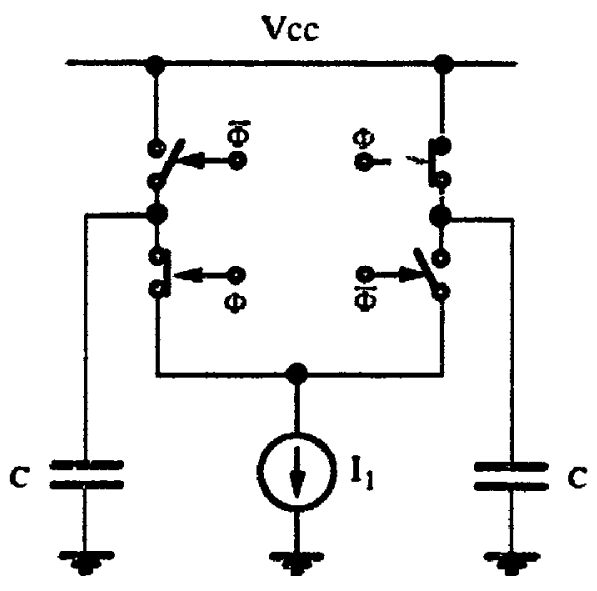

Figure 3.10 A high-speed ground capacitance modified emitter coupled oscillator. 


\subsubsection{Noise considerations}

Due to the lack of frequency selective networks (LC or resonators), ring oscillators usually exhibit a poor phase noise performance. In comparison to RC relaxation oscillators, the use of a current source approach in a constant-current relaxation oscillator seems to be a solution that provides better phase noise. This scheme allows higher switching consistency on the timing capacitor. Thus, in most low-noise oscillator designs, a current-controlled oscillator (ICO) is preferred.

\subsubsection{3. $Q$ of $\mathrm{CMOS}$ relaxation oscillators}

As mentioned earlier, the multivibrator type oscillator has only one energy storage element, i.e., a capacitor, which, by its nature results in a low- $Q$ oscillator. In comparison to LC oscillators, the $Q$ of a multivibrator is $1 / 4$ [44].

One of the primary goals in the CMOS VCO design was to obtain a high Q factor without the use of tank circuits. The use of inverter integrators in a ring oscillator can provide higher $Q$ than a regular single stage relaxation oscillator. This section discusses the enhanced $Q$ obtained by using a multi-stage multivibrator approach.

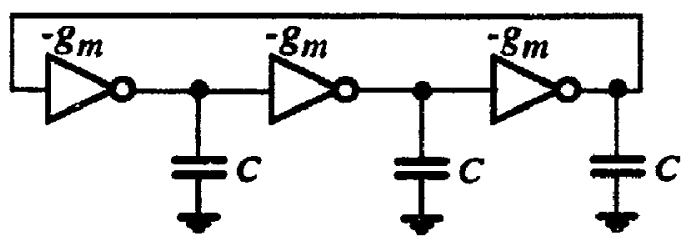

(a) Three-stage ring inverter as multivibrator

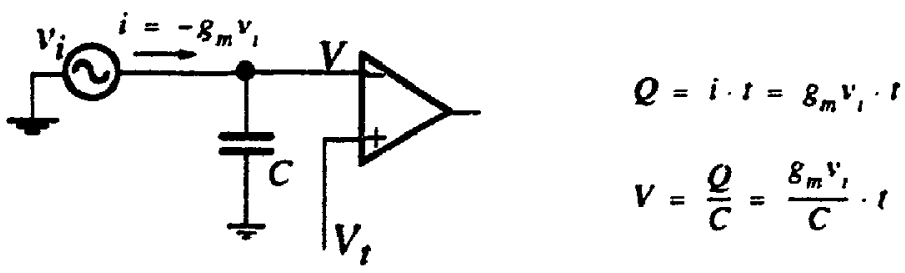

(b) Linearized model for one stage multivibrator

Figure 3.11 Ring oscillator linearized model. 
A multivibrator can be modelled by looking at its corresponding linear transfer function [44], theiefore the cascaded inverters that form a ring oscillator can be modelled as linear integrators. The effect of integration is illustrated in Figure 3.11. One inverter combined with its timing capacitance (assuming all $g_{m}$ components and $C$ are identical) is modelled as shown in Figure 3.11.b.

For the first order approximation, assume that $v_{i}=V_{t}-V$ and let $T$ be the delay in each integrator stage, then

$$
T=\frac{C}{g_{m}}
$$

and from the Laplace delay operation $e^{-s T_{\text {delay }}}$, therefore delay $T$ is represented by the delay operator, $e^{-s C / g_{m}}$. This can be expanded, using the Taylor series

$$
e^{-s \frac{C}{g_{m}}}=1-\left(s \frac{C}{g_{m}}\right)+\frac{1}{2}\left(s \frac{C}{g_{m}}\right)^{2}-\frac{1}{6}\left(s \frac{C}{g_{m}}\right)^{3}+\ldots
$$

Figure 3.11.a can be redrawn using a signal flow graph as shown in Figure 3.12.

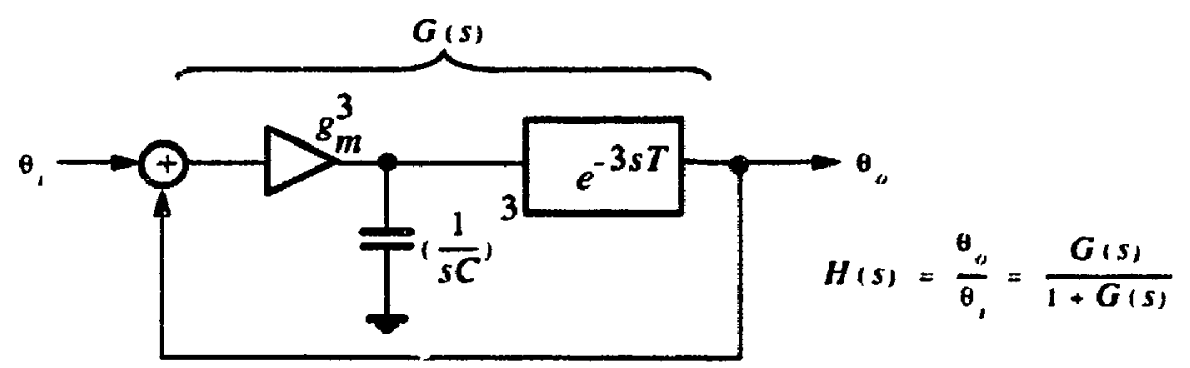

Figure 3.12 Linearized $1^{\text {st }}$ order model for three-stage multivibrator.

From Eq. 3.11, neglecting the terms with order higher than two, the characteristic equation, $1+G(s)$, of the model in Figure 3.12 is given by 


$$
1+G(s)=1+\left(\frac{g_{m}}{s C}\right)^{3} \cdot\left(1-s \frac{C}{g_{m}}+\frac{1}{2}\left(s \frac{C}{g_{m}}\right)^{2}\right)^{3}
$$

The roots of the above characteristic equation indicate three pole pairs. The circuit can therefore be optimized to achieve a placement of a dominant pair of poles corresponding to a much higher $\mathbf{Q}$ than that of a simple multivibrator. This issue is not discussed further in this thesis and is currently the subject of interest within the VLSI in Communications Research Group.

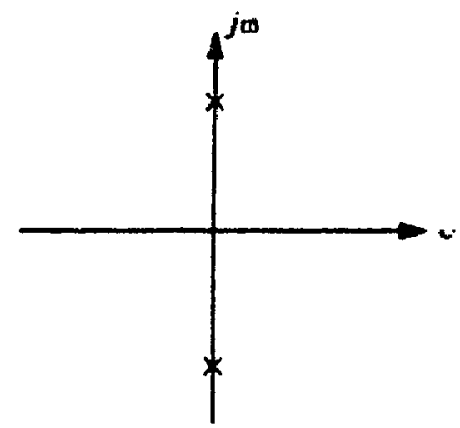

(a)

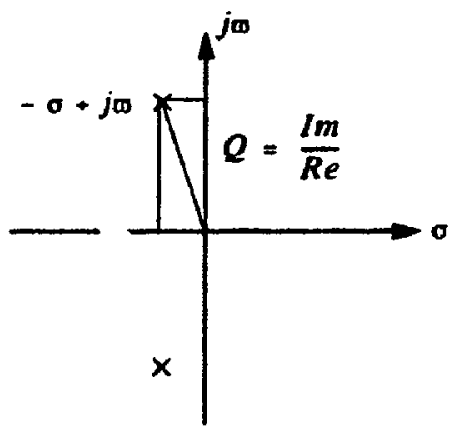

(b)

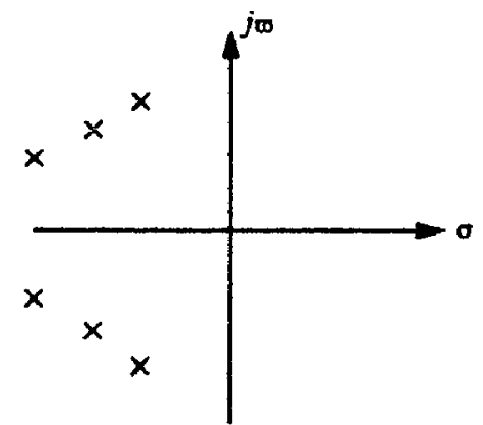

(c)

Figure 3.13 Oscillator poles on complex frequency plane (a) Ideal case, (b) LC with losses (c) Multi-stage multivibrator.

The oscillator output phase noise is proportional to the magnitude of the noise sources present (circuit, substrate injected) while simultanecusly being inversely proportional to the oscillator $Q$ [44]. Since circuit optimization and layout for a given architecture influence circuit intrinsic noise sources, substrate injected noise and pole pair placement $(Q)$, the circuit optimization is not a trivial task.

Some methods to minimize noise injection include:

- Filtering the VCO power supply

- Shielding the VCO circuit

- Filtering the controlling voltage to the VCO 
- Buffering the VCO output

- Eliminating ground loops that would include the VCO.

\subsubsection{The analysis of CMOS oscillator in the time domain}

The basic design of a CMOS oscillator, either an inverter ring oscillator or latching oscillator, can be described by a time domain analysis. An inverter ring oscillator is an ideal example for analyzing the circuit operation.

An approximate linear time domain model can be developed because the time constant (t) of each inverter stage is known, so the transfer function of each integrator can be described by $h(t)=e^{(-t / \tau)}$. This assumes that $\tau$ is not only dependent on the geometry of each transistor but also on the dynamic operation of the system.

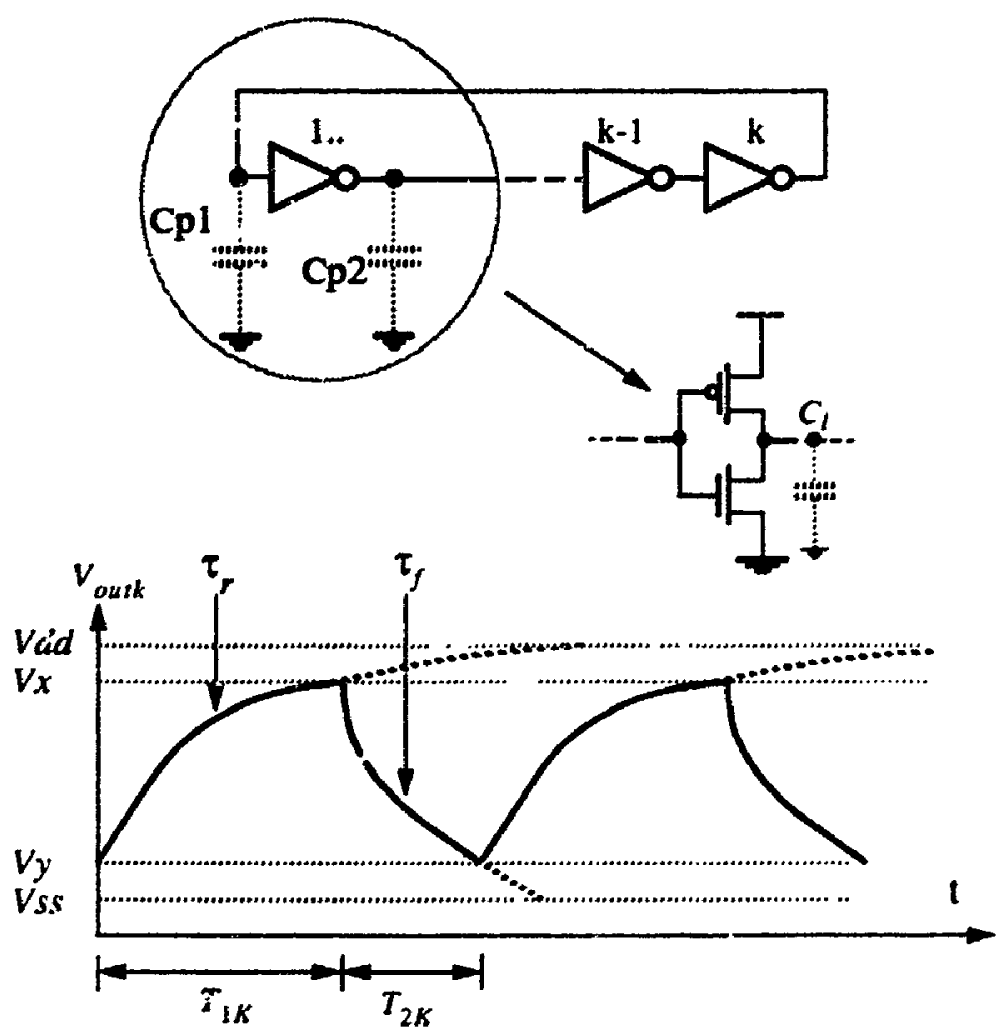

Figure 3.14 Timing diagram of RC relaxation oscillator. 
The oscillator consists of an odd-number inverter ring (K-stage). By assuming each inverter as a linear gain block with the threshold voltage of both $\mathrm{n}$ and $\mathrm{p}$ MOS devices defined by $V t h=(V d d+V s s) / 2$, thus the output, Voutk, changes state when the increasing level of $V$ in reaches an uppe: level switching threshold, $V x$. It then reverts to its initial state when the input falls to a lower threshold level, $V y$.

A i.st order time domain analysis defines the frequency of oscillation and operating state of each inverter stage. It can be seen in Figure 3.14 that the oscillator's timing control is comprised of two states, a charging state, $T_{1 k}$ and discharging state, $T_{2 k}$. These states are determined by the rising time constant, $\tau_{r}$ and falling time constant, $\tau_{f}$. In the inverter ring oscillator, $\tau_{r}$ and $\tau_{f}$ are determined from the $\mathrm{n}$ and $\mathrm{p}$ MOSFET characteristics and the additional parasitic resistances and capacitances of the inverter.

The output $V_{\text {outK }}$ of each inverter stage, expressed as a function of the input charging state, is given by

$$
\begin{aligned}
& \text { Vout }_{k}=V d d-\left(\left(V d d-V_{y_{k}}\right) \cdot e^{-\frac{t}{\tau_{r k}}}\right) \\
& \text { Vout }_{k}=V s s-\left(\left(V s s-V_{x_{k}}\right) \cdot e^{-\frac{t}{\tau_{j k}}}\right)
\end{aligned}
$$

Hence, the time period $T_{1 k}$ and $T_{2 k}$ taken by charging and discharging are given by

$$
\begin{aligned}
& T_{1 k}=\tau_{r k} \ln \left(\frac{V d d-V y_{k}}{V d d-V x_{k}}\right) \\
& T_{2 k}=\tau_{f k} \ln \left(\frac{V s s-V x_{k}}{V s s-V y_{k}}\right)
\end{aligned}
$$


The total period of the oscillator $T$ is equal to $T_{l k}+T_{2 k}$, and the oscillating frequency, $f$, is given by

$$
f=\frac{1}{T}=\frac{1}{\left(T_{1 k}+T_{2 k}\right)}
$$

Equations 3.15 to 3.17 provide a means of sizing the devices for an inverter ring oscillator design in order to achieve the desired frequency of oscillation. However, at high frequencies greater than $100 \mathrm{MHz}$, parasitics begin to have significant effects and the accuracy of a time domain analysis becomes unreliable. This is due to the difficulty in obtaining exact device parameters for high-speed operation. More accurate analysis can be attained by considering a full representation of each inverter stage with a dynamic load capacitance, as shown in Figure 3.15.

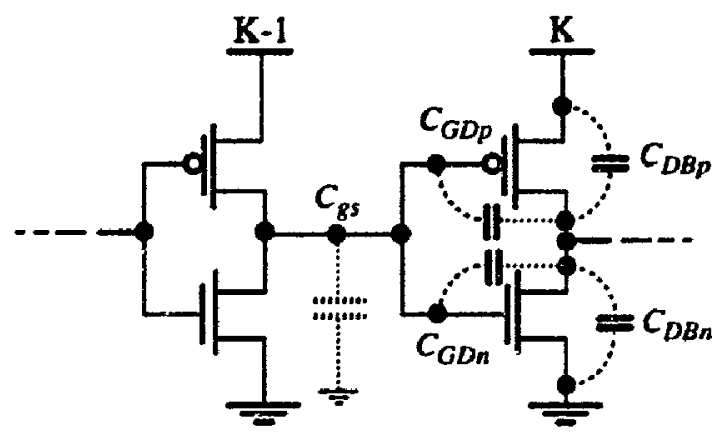

Figure 3.15 MOS inverter with dynamic load capacitance in ring oscillator.

This well-known configuration is used as a basic concept for determining the speed of a CMOS inverter [59]. The analysis is based on the fact that load capacitance is a function of device geometry. The load capacitance of the inverter stage K-1 is the total input capacitance of the subsequent stage $K$. This load capacitance is made up of two dominant components, gate capacitance $\mathrm{Cgs}$ of stage $\mathrm{K}$ and the Miller effect transconductance, a function of both $\mathrm{K}$ and $\mathrm{K}-1$ stages. 
The effect of the dynamic operation of the inverter when connected in a ring structure makes the time domain analysis even more complex because of the interaction of the closed loop inverters. Even if approximated time constants $\tau_{r}$ and $\tau_{f}$ can be calculated by making an assumption that Vgs for all MOS devices is at midpoint, this would only be an approximation at best. Furthermore, due to the nonlinear nature of the switching system as was the case for the relaxation oscillator, the calculated values of $\tau_{r}$ and $\tau_{f}$ are always inaccurate. As a result, there is an accuracy limitation for obtaining the exact values of all capacitances and resistances in a dynamic inverter ring oscillator that operates at very high frequencies. Further modelling and analysis is being undertaken within the TRIO group and will be presented in 1994-5. This requires the development of an extensive numerical optimization tool. However, a new technique developed in the course of the CMOS VCO work allows simulation in the frequency domain, for optimization of speed and phase noise. This technique is presented in Chapter 4.

\subsubsection{Techniques for the design of a low-noise oscillator}

Noise in relaxation oscillators can be improved with various techniques both at system and circuit levels. This section provides viable architectures and techniques that are used in most oscillator designs.

\subsubsection{Regenerative feedback}

As explained in Section 3.2.2.4, the limited switching speed of the inverting devices is a major source of phase noise. Since the switching characteristics of a CMOS inverter in a relaxation oscillator are determined by device sizing, design of a fast inverter requires optimization. When an optimized layout has been achieved, switching speed can be further enhanced with the use of a regenerative network.

Regenerative feedback realized with two inverters connected front to back, as shown in Figure 3.16. is common in fast memory circuits, specifically sticky latch circuits. 
The regenerative process occurs quickly due to the positive feedback [60]. If the latch is applied in a ring oscillator and controlled so that no stable condition takes place, oscillator noise will be reduced.
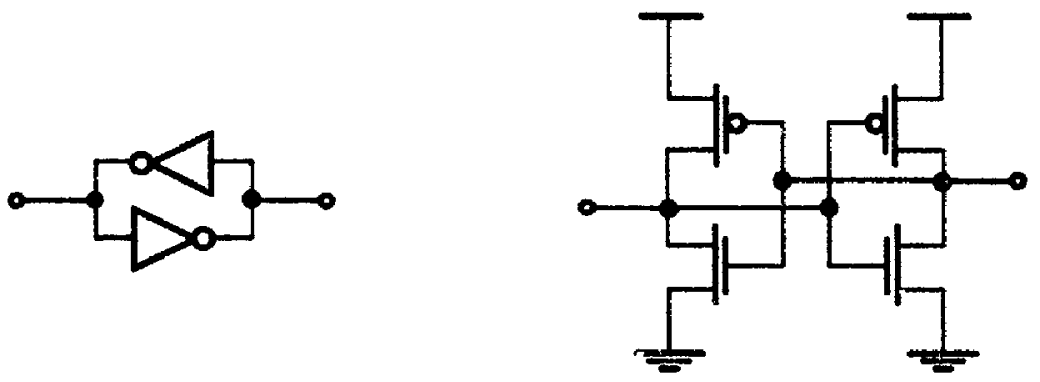

Figure 3.16 Regenerative network using a two-inverter latch

\subsubsection{Differential structure}

Differential circuit configurations are well known for their common mode rejection ratio over alternate single ended techniques (Figure 3.14). At the expense of twice the circuit size and complexity, the differential ring oscillators offer improved noise performance. However, design trade-offs should be taken into account because increased circuit complexity results in a speed and power penalty.

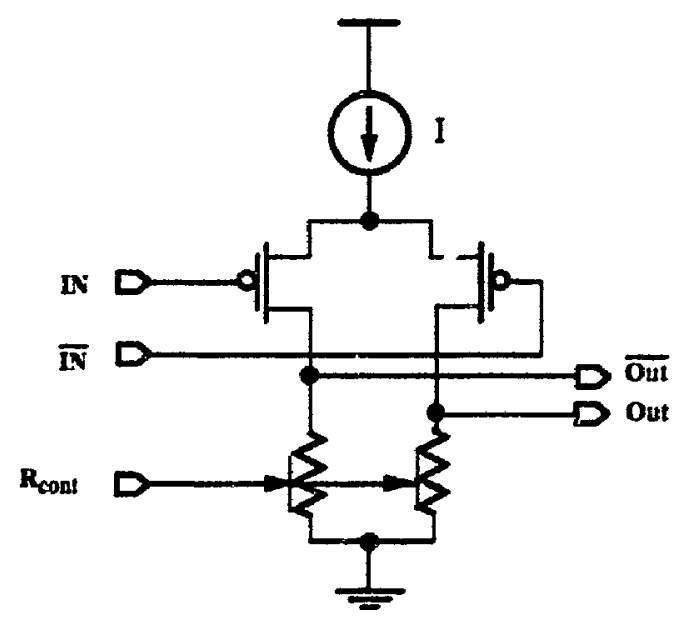

Figure 3.17 Differential delay element [35] 


\subsubsection{Circuit partitioning}

In highly integrated mixed analog-digital circuits, care must be taken to reduce the effects of cross coupling noise caused by digital switching currents. Such noise can be coupled into analog parts such as the VCO and loop filter. Circuit layout plays a significant role in minimizing the noise of mixed signal architectures.

\subsection{The design of a PLL frequency synthesizer}

To successfully pursue the idea of integrating all the PLL components on a single die, other loop elements are required. These include the phase detector and loop filter, and the frequency divider.

\subsubsection{Phase detector and loop filter}

The maxinun input frequency of the phase detector and loop filter can be, by design, significantly lower than the maximum oscillator output frequency. However, in order to improve the overall performance of the synthesizer, the key design considerations associated with each function should be considered closely. Because a CMOS implementation is the most desirable approach, this section also addresses the possibility of using both an integrated phase detector and loop filter sirce both lend themselves to monolithic realization.

\subsubsection{CMOS phase detector}

The function of the phase detector is to provide an average output voltage proportional to the phase difference between the stable reference signal and the divided down VCO output (see Section 3.1). PLL synthesizers require phase detectors to have:

- Wide phase detection range

- Good linearity and low distortion

- Low power dissipation

- Small size for integration. 
Although conventional tri-state CMOS phase-frequency detectors are the most popular for many monolithic PLL designs, the existence of a phase distortion zone or socalled "dead zone" can lead to PLL spurious noise problem, degrading the overall performance of the synthesized signal (detailed analysis in [6] and [61]).

A phase detector structure which mitigates this problem is shown in Figure 3.18. The configuration is based upon an extended range exclusive OR (XOR) with a frequency discriminating circuit [62].

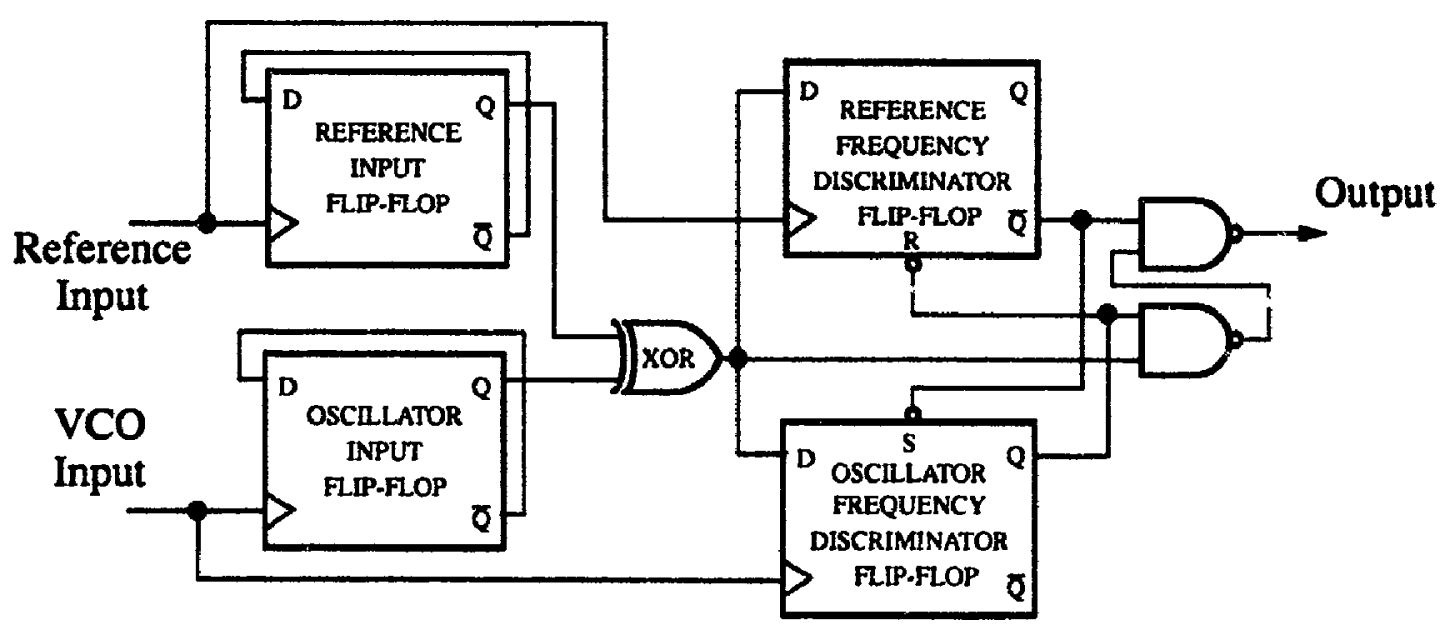

Figure 3.18 Phase-frequency detector with no dead zone.

This structure delivers a $360^{\circ}$ detection range and eliminates the dead zone by moving it to the ends of the detection range. When incorporated in a PLL synthesizer, this results in the phase detector operating in the linear region for the lock condition.

Frequency detection in this type of phase-frequency detector overrides the phase detection function whenever the reference input and the VCO input are different in frequency (i.e., a time varying average phase error voltage). This ability helps in pulling the VCO output frequency back to a stable locked condition, allowing a small lock in time. 
Most mobile radio frequency synthesizers employ a reference f:equency of no more than $50 \mathrm{MHz}$ since high-frequency crystal oscillators are expensive and have high phase noise to be used as a reference source in practical implementation. Since the phasefrequency detector does not require performance in speed, it can easily be implemented with CMOS digital standard cells.

\subsubsection{Loop filter}

By filtering the pulse train output from the phase-frequency detector, as explained above, an average dc output is attained that can be used to drive the VCO, forcing the PLL synthesizer to operate in the lock mode.

The loop filter can be as simple as a first-order RC passive network or as complex as $a 4^{\text {th }}$ order structure, depending on the critical parameters of the application. Higher order loop filters are certainly better at ac filtering, though at the cost of circuit complexity and the risk of instability [63].

In any attempt to integrate a loop filter on chip, issues of concern include accuracy of the pole frequency position and $\mathrm{Q}$ factor, loop filter noise sensitivity (to the digital ground), and most importantly, power efficiency.

A CMOS implementation of a fully integrated loop filter has been detailed by Plett [64]. Several approaches suited to rnonolithic design (Figure 3.19) are summarized briefly here. 


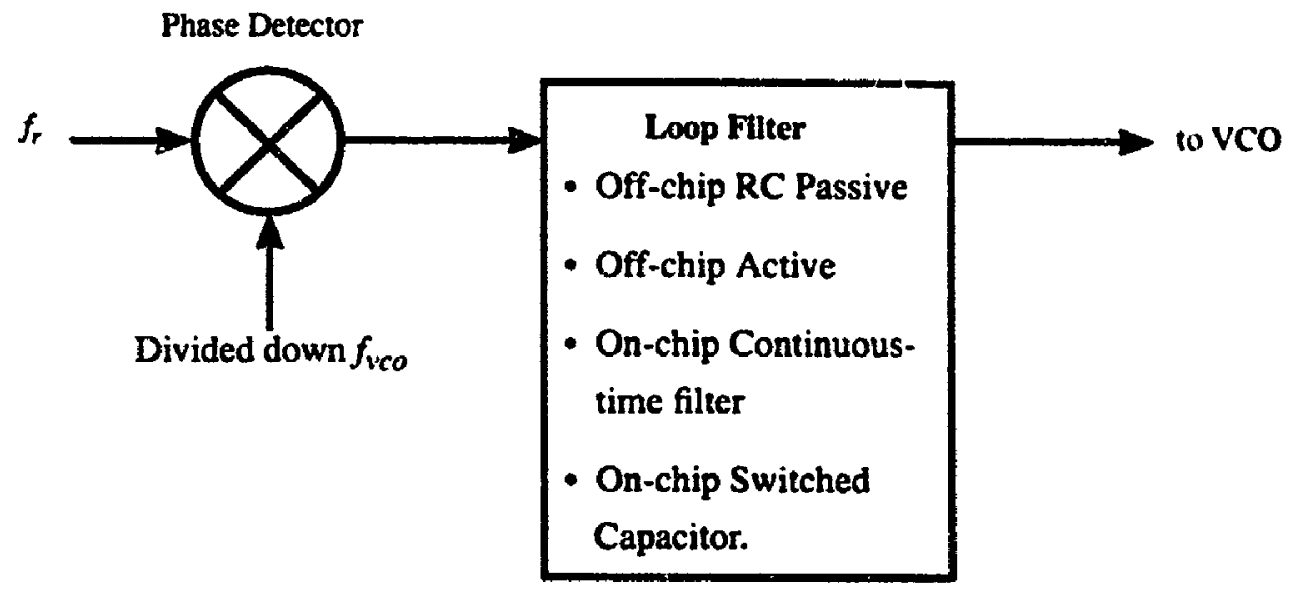

Figure 3.19 Choices of loop filter implementation.

Continuous-time filters are likely to have advantages over switched capacitor filters in their superior noise performance (i.e., better dynamic range). They can be implemented as a circuit without the use of opamps and can be tunable to the required frequency and $Q$ by employing a scheme of additional automatic tuning circuits.

However, since a large time constant for ac filtering is necessary, large capacitors on-chip are unavoidable. Power consumption can be a major obstacle to building a tunable on-chip loop filter for low-power purposes. In this case, a switched capacitor filter may be a good choice if clock feedthrough problems can be resolved.

\subsubsection{Fully balanced phase-frequency detector and loop filter}

For *' hest possible noise performance, a differential structure is recommended $[65,66]$. Differential operation of the phase detector can be accomplished by replicating the single ended circuit and cross-coupling the two inputs. In this manner, a balanced input iuop filter is required, as shown in Figure 3.20. 


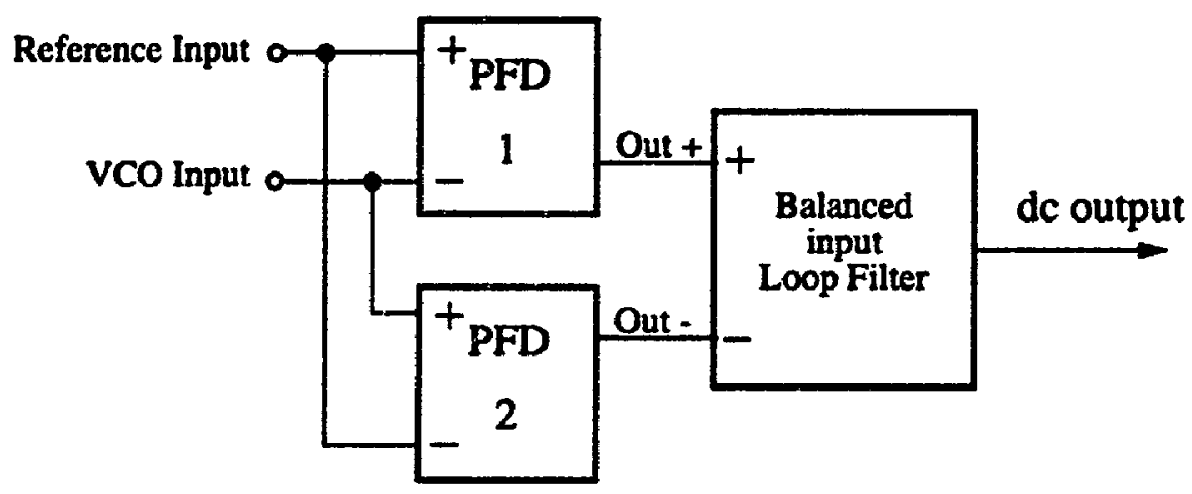

Figure 3.20 Fully balanced configuration of phase detector and loop filter.

The crucial benefit of having a balanced structure is that the amount of common mode jitter in the zero crossings at the PFD output due to substrate noise will be cancelled out at the differential-input loop filter. As a result, he structure provides high immunity to noise coupled from digital circuitry.

\subsubsection{Frequency divider}

High-frequency CMOS fractional-N synthesizers have been successfully achieved at speeds up to a few megahertz as reported in [5] and [67]. The design of a dual modulus divider proposed by $\mathbf{N}$. Foroudi [5] has the highest potential as an integral part of a monolithic CMOS synthesizer both for speed and power consumption. In addition, the design is well suited to a conservative scalable standard CMOS process for fabrication, offering better cost effectiveness and reduction in the development time for future improvements.

If a $900 \mathrm{MHz}$ frequency range synthesizer is considered, a 15/16 modulus divider can be directly used, allowing for a CMOS phase-frequency detector as described in the previous section. The divided down VCO output frequencies can range from 50 to 60 $\mathrm{MHz}$ and are suitable for the proposed divider. 


\subsection{Summary}

This chapter has pointed olt several key design considerations for a high-speed lowpower PLL frequency synthesizer at both the system and component levels. A number of issues associated with limiting factors in conventional monolithic VCO design have been described. It has been demonstrated that CMOS oscillators in the past lacked the speed and noise performance necessary for use in a mobile radio frequency synthesizer. However, structures that accommodate and improve CMOS oscillators for high speed as well as low noise still exist if proper design principles are utilized. First order time domain analysis of parasitic-capacitor-based CMOS VCOs and their contribution to noise jitter in the relaxation oscillator have been presented. An obstacle in achieving adequate accuracy of time domain analysis, primarily caused by the intricate dynamic operation of a highspeed inverter ring oscillator, has been illustrated. Lastly, the realization of a fully integrated CMOS synthesizer has been explored and proposed.

The next chapter presents the detailed design of high-speed CMOS VCO architectures that operate with the use of their circuit parasitics. A novel approach for relaxation oscillator analysis in the frequency domain is introduced, along with the results of new optimized CMOS VCO designs. These are believed to be the fastest and to have the lowest phase noise in their class and are very likely to be the best suited for single-chip synthesizer solution. 


\section{Chapter 4}

\section{The Design and Implementation of a CMOS Frequency Synthesizer}

Low power consumption ard high frequency operation are the critical parameters that must be achieved for suciess in frequency synthesizer design. These requirements are even more challenging for a CMOS implementation.

This chapter presents a design for an all CMOS high-speed PLL synthesizer. Emphasis is placed on the knowledge required to successfully design a CMOS VCO that achieves both speed and noise performance as presented in Chapters 2 and 3. The foremost design goals are (i) to develop new simulation techniques that support the design of parasitic-capacitor-based CMOS VCOs that produce better accuracy and quality and (ii) to introduce CMOS VCOs that have potential for high-speed operation. As a result, the first topic discussed is the proposed ac MOS model for a CMOS oscillator, followed by three newly developed on-chip oscillator architectures that maximize the operating speed of the CMOS process. 
Relevant design issues associated with improvements to noise and layout optimization are also addressed, as well as simulation results of the proposed VCO structures accompanied by the validation of developed simulation techniques. It is shown that the proposed VCOs meet the requirements of low power consumption and. in future with a shorter gate-length process, could provide a maximum frequency up to several gigahertz.

The VCO layouts and corresponding transistor sizes are presented in Appendix A. CMOS4S process parameters used for both simulations and chip implementation are given in Appendix B.

\subsection{Linear ac model for îrequency domain analysis}

As described in the previous chapter, the accuracy of a first order time domain analysis for a generalized high frequency $\mathrm{RC}$ relaxation oscillator is limited in its ability to represent true oscillator behavior. For this reason, a frequency domain analysis of the relaxation oscillator is explored. The objective is to quantify the oscillator's performance parameters by building models based upon symbolic representation of the oscillator's device parameters (W/L, layout parasitics, etc.).

The resulting small-signal linearized circuit analysis provides several advantages over an alternate time domain investigation. First, the system bandwidth can be controlled in the design. This relates directly to the $Q$ factor of the oscillator, which is circuit and device dependent. Second, it provides design flexibility which allows for a custom design of oscillator center frequency. All of these advantages result in the design of oscillators that are more reliable and less complex, and allow performance parameters to $\&$ evaluated before actual implementation.

The frequency domain model of Figure 4.1 is based upon a conventional ac linear model found in most control systems. The model can be best understood by thinking of the oscillator as a simple linear gain block connected in a feedback configuration. 


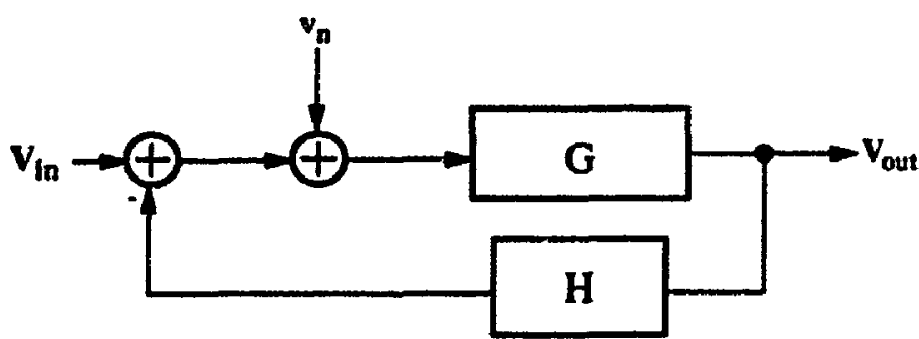

(a)

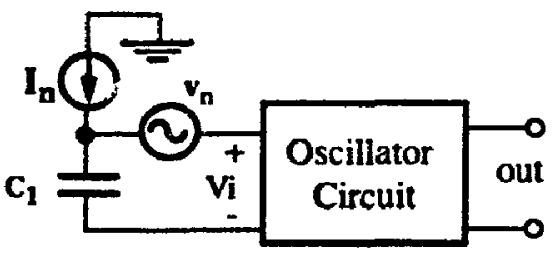

(b)

Figure 4.1 (a) Basic block diagram for representation of VCO in frequency domain, (b) Model of an injected noise current for actual analysis in oscillator circuit ( $\mathrm{Vn}$ - noise inyut voltage).

In the case of an ideal oscill-tor system (referred to as an ideal filter with a component. being a part of the VCO), the frequency selective behavior of the oscillator allows only the operating frequency to propagate to the output. In practice, the oscillator has limited $\mathbf{Q}$ which results in a certain level of noise rejection over the entire frequency range. Thus, noise within the vicinity of the oscillator's center frequency is not suppressed [68].

The equivalent noise, which can be an injected white noise as in the current source $I_{n}$, shown in Figure 4.1.b, at the input of the circuits, adds to the linear voltage waveform on the timing capacitor $C_{1}$, which could result in an uncertainty in the time of regeneration as introduced in Section 3.2.2.4.

With such a linearized model, it is possible to realize az oscillator with an arbitrary transfer function. However, for such an approach to be successful, one needs to develop relevant linearized models for MOS transistors.

\subsubsection{Proposed MOS model for ac analysis}

The realistic ac model used for small-signal frequency domain analysis directly relates system sensitivity to noise sources. Such an analysis allows one to simultaneously explore the dynamic operation of the oscillator and its sensitivity to different device parameters. Such analysis can replace the complex, somewhat inaccurate, time domain evaluation (Section 3.2.5). 
The main objective of the VCO analysis in the frequency domain is to be able to control the change of the VCO's performance parameters. Parameter variation is achieved by differing device sizes and layout configurations. One of the tools that allows the mathematical analysis of circuits with symbolic representations is NODAL ${ }^{\prime}$ [69]. NODAL is a CAD tool which works in conjunction with another symbolic-basedoperation environment, Mathematica ${ }^{\mathrm{TM}}$ [70]. With a CMOS VCO netlist whose MOS device models have been replaced by newly developed MOS model subcircuits, it is possible to symbolically analyze various oscillator circuits. This allows determination of oscillator characteristics such as (i) the location of pole frequencies, (ii) pole quality factor or $\mathbf{Q}$ and (iii) circuit sensitivity to device and process parameters. These tools allow easier design and customization of VCOs to be used in specific applications.

\subsubsection{Model derivation}

In a conventional ac analysis using the SPICE simulator, two problems were encuuntered. First, SPICE simulates a give.a circuit depending on the assigned bias point, and therefore to obtain reliable results all transistors in the circuit must achieve stable vias points (dc convergence). Since the oscillators developed in this work are nonlinear in nature (i.e., switching carcuits), when SPICE determines the $g_{m}$ of each transistor, this is the $\mathrm{g}_{\mathrm{m}}$ of the transistor in the switching state. Hence, the resulting simulated frequency of oscillation is always higher than those from the transient analysis. This is due to the extremely high $g_{m}$ values that SPICE obtains from the switching state. Secondly, SPICE does noi account for two states in which the inverter in the ring operates, the saturation mode and the off mode. These two states result in minimum $\mathrm{g}_{\mathrm{m}}$ values. Therefore the switching behavior inhibits correct ac analysis with the models provided in SPICE.

1. NODAL ${ }^{\text {TM }}$ is a trademark of Macallan Consulting. 


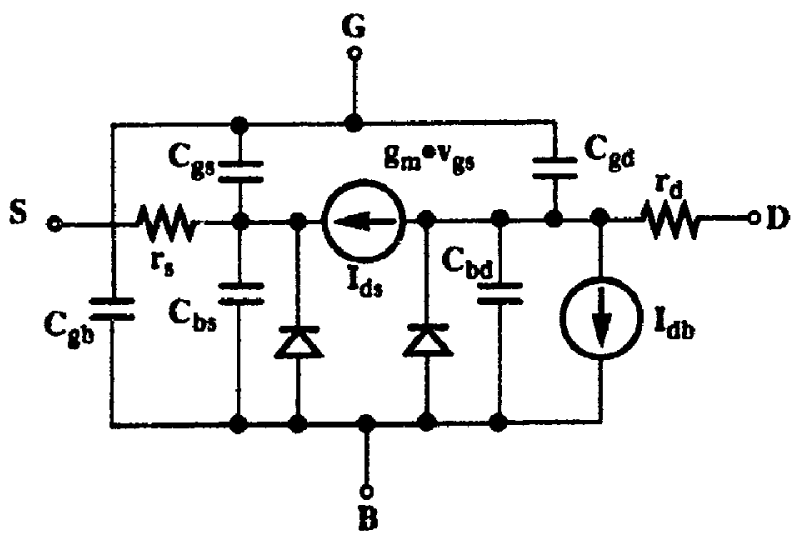

a) Transient Analysis

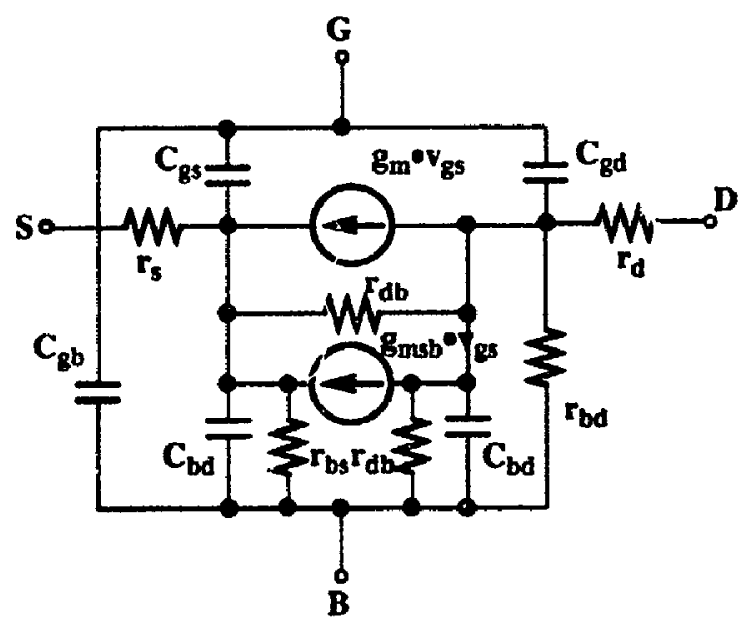

b) AC Anaiysis

Figure 4.2 HSPICE equivalent circuit MOSFET [71].

To be able to perform a proper ac analysis that delivers a reliable accuracy, a new linearized model has been developed. The proposed analytical model is based upon the HSPICE MOS device model [71], as shown in Figure 4.2. The idea is that with the linearized model inserted in place of each transistor in the relaxation oscillator, a result similar to the actual SPICE transient analysis can be gained i.e., a result of an oscillating frequency that more closeiy matches the frequency determined by a SPICE time domain analysis.

Linearization was obtained by taking average values of MOSFET parameters, using reasonable assumptions for the operating point of MOS transistors. Since $\mathrm{g}_{\mathrm{m}}$ is a parameter that is a function of biasing voltage, maximum $\mathrm{g}_{\mathrm{m}}$ results at $\mathrm{V}_{\mathrm{gs}}$ equals 5 volts for NMOS and -5 volts for PMOS (assuming the full swing of the CMOS inverter). The model was linearized by removing the diodes, as shown in Figure 4.2.a. (For a detailed analysis see [72]). An acceptable approximation was achieved through several simulicion trials, by setting the operating point for ac analysis at midpoint i.e., $\mathrm{V}_{\mathrm{gs}}$ equal +2.5 and -2.5 volts for NMOS and PMOS transistors respectively. At these voltages, it is certain that both transistors are fully on. resulting in approximated linear values for elements $g_{m}, C_{b d}$ and $C_{b s}$. 


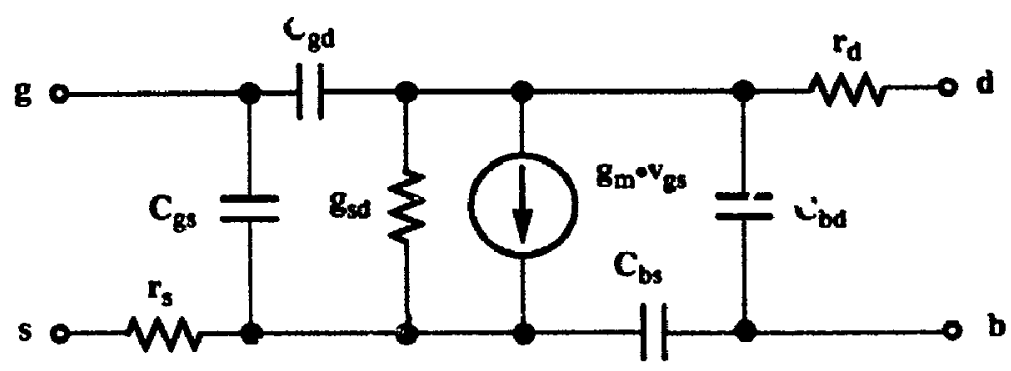

Figure 4.3 A proposed linear ac model for MOS devices in oscillator circuits.

Some of the elements shown in Figure 4.3 are bias dependent, while Cgd and Cgs are a function of the device operating state (on, switching, off). The worst case linearized value [72] which leads to a conservative result (high side capacitance) estimates $C_{g d}=C_{g s}$ $=2 / 3 C_{g}$.

The approximate model takes into account the contribution of all components that make up the model. The ideal linearized model should also account for ail of the parameters in the practical model. However, from a large number of simulations, the parameters $g_{m s b}, r_{b s}$ and $r_{d b}$ (shown in Figure 4.2.b) are shown to insignificantly influence the operating point of the oscillator in terms of the movement of pole location. Therefore, the derivation of the first model does not include these parameters in the approximation, and the linearized model is therefore simplified. Figure 4.3 shows the resulting ac MOS model for both $\mathrm{n}$ and $\mathrm{p}$ type MOS devices.

As shown in Section 4.4, the simulation results based on the linear ac MOS model do not correspond to the exact HSPICE transient analysis. For example, a slight shift of the oscillator's center frequency to a lower frequency occurs due to the approximation error of model parameters is difficult to forecast (i.e., circuit state dependence). The results and validation of this proposed model are described after several newly developed highspeed CMOS VCOs are presented in the next section. 


\subsection{High speed CMOS oscillator design}

Table 4.1 summarizes the development of various on-chip PLL synthesizers and VCOs implemented in CMOS processes to date.

\begin{tabular}{|c||c|c|c|c|}
\hline Sources & Application & $\begin{array}{c}\text { Gate Length } \\
(\mu \mathrm{m})\end{array}$ & $\begin{array}{c}\text { Max. Frequency } \\
(\mathrm{MHz})\end{array}$ & $\begin{array}{c}\text { Consumed Power } \\
(\mathrm{mW})\end{array}$ \\
\hline \hline$[37]^{\prime} 93$ & PLL Clock Gen. & 0.7 & 266 & - \\
\hline$[38]^{\prime} 93$ & PLL Clock Gen. & 0.8 & 330 & - \\
\hline$[36]^{\prime} 93$ & PLL Clock Gen. & 0.9 & 690 & - \\
\hline$[73]^{\prime} 93$ & PLL Clock Gen. & 1.2 & 480 & - \\
\hline$[35]^{\prime} 92$ & $\mu$ P Timing Gen. & 0.8 & 220 & - \\
\hline$[34]^{\prime} 92$ & Video Graphics & 1.2 & 180 & - \\
\hline$[55]^{\prime} 92$ & VCO & 1.2 & 80 & 20 \\
\hline$[53]^{\prime} 90$ & VCO & 1.0 & 325 & - \\
\hline
\end{tabular}

Table 4.1 High-speed CMOS oscillators as parts of PLL applications and separate VCO circuits.

This indicates that the speed of CMOS devices is significantly improved with shorter gate-lengths. However, the maximum oscillating frequency of the reported on-chip VCOs do not meet the requirements for digita! mobile communications. As well, phase noise figures are not reported for any of the devices listed in the Table 4.1.

The key issues addressed in this chapter are (i) the possibility of improving the speed of the CMOS VCO up to gigahertz frequency without a special submicron process, and (ii) the ability to characterize and predict VCO performance before implementation. Success in the latter enables one to select and customize the VCO for best performance to maximum speed.

Based on analysis of the CMOS nscillator introduced in Chapter 3, CMOS can be used to build fast oscillators by using simple structures such as ring oscillators or currentcharge-discharge latching circuits. The first approach is suitable for high-speed operation 
but is difficult to precisely characterize. The latter works better in a frequency control configuration, constructed to control a current source supplying the core oscillator circuit.

Yet, regardless of the oscillator structure, to successfully realize high speed and low noise, a deeper understanding of optimum design is required.

\subsubsection{Speed vs. noise design}

As described in the previous chapter, the speed limiting factors of relaxation oscillators are the timing capacitances and the speed of the switching devices used in the oscillator. Reducing timing capacitance can increase the oscillating frequency at the expense of losing the certainty of the timing edge of the switching waveform, thus affecting the phase noise performance. As a result, this approach is not a viable option.

Alternatively, if the switching speed of the active device is to be considered, the significance of its influence on the oscillator's phase noise must be determined. Using the fastest possible device will certainly enable the oscillator to operate at higher frequencies as well as reduce its susceptibility to waveform uncertainty across the timing capacitor. If both speed and noise are to be achieved, the optimum oscillator design should give priority to speed improvement in switching elements.

Since a speed-optimized structure is selected as the key design objective required to obtain high-frequency and low phase noise, then a focused effort will be made to maximize the speed of the oscillator for the specific process. In addition, to further improve noise performance, the following techniques will be incorporated:

- Employ a switching waveform with sharpest rising and falling edge [46]

- Balance timing of the waveform for a 50\% duty cycle [35]

- Minimize the number of stages for the ring oscillator [74]

- Use a symmetrical layout design [52]. 


\subsubsection{Modified ring oscillator}

In a simple inverter ring oscillator, the control of the oscillating frequency is achieved by varying a delay at one of the inverters embedded in the ring. However, traditional design usually employs a cascade MOS device as a delay cell to control the resistance across the chain (see Figure 4.4) [35]. Consequently, the oscillator produces negative transfer (voltage-to-frequency) characteristics. This results in an adverse effect for applications in a phase locked loop since the minimum frequency is limited by the maximum voltage on the power supply.
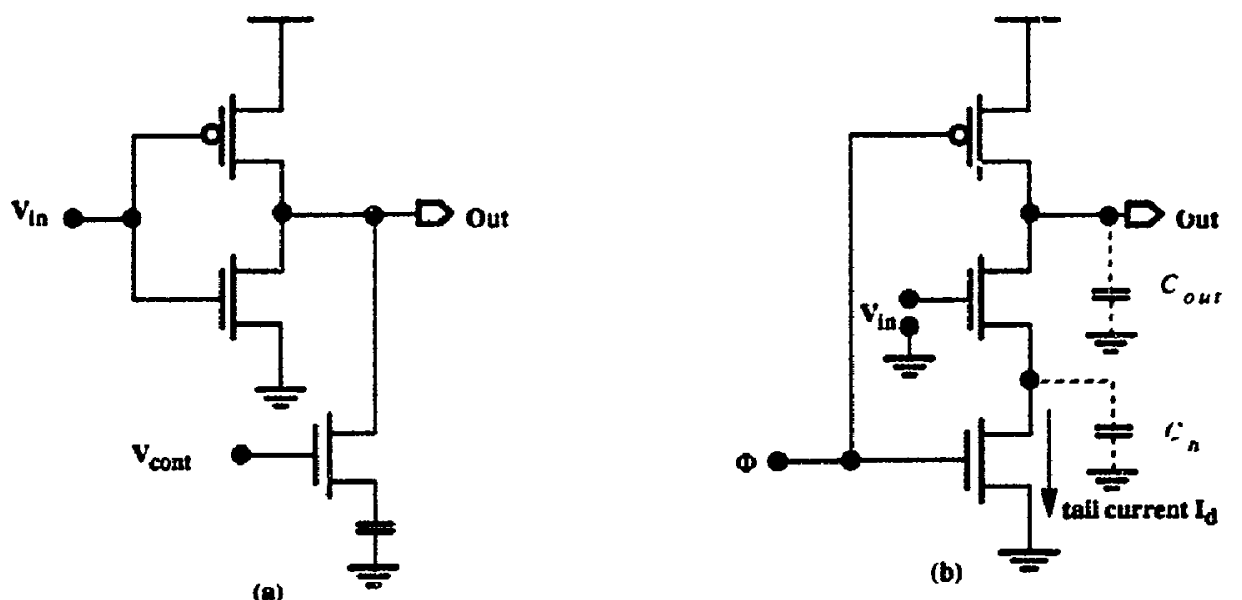

Figure 4.4 (a) basic delay cell and (b) dynamic NMOS inverter as variable inverting delay cell.

A way around this problem is to use a current-control-frequency scheme that employs a voltage-to-current converter (V/I) to control the current that charges and discharges the timing capacitor. This method provides positive VCO gain at the expense of higher circuit complexity and power consumption.

A new inverter architecture, introduced in this work, is based upon a modified threetransistor dynamic NMOS inverter and is shown in Figure 4.4.b [59]. By rearranging the clock input and $\mathrm{V}_{\mathrm{in}}$, a variable delay element can be achieved. 
Direct control of the current through the inverter stage is achieved through variation of the gate voltage which in turn controls the tail current $I_{d}$ of the inverter. If the capacitances, $C_{\text {out }}$ and $C_{n}$ are constant, the delay is inversely proportional to the variable tail current. The key advantage of using a dynamic CMOS inverter is that it provides faster switching speeds without incr-asing circuit complexity.

The factor that affects linearity of a simple inverting delay element is the saturation region as modelled for a square law device. Current through a MOSFET cannot be increased when the bias of a MOSFET approaches saturation mode. However, shortchannel MOS (gate-length less than $3 \mu \mathrm{m}$ [59]) effects result in a gradual positive increase in the current $I_{d}$ that is proportional to $V_{d s}$, as illustrated in Figure 4.5.

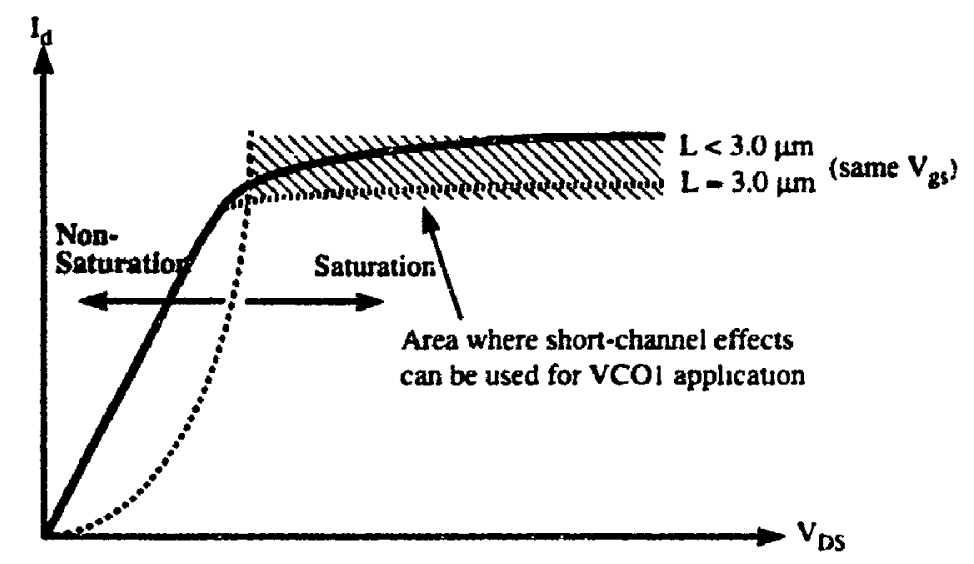

Figure 4.5 Short-channel effect in the MOSFET saturation morie and its suitable application for VCOI (shaded area is in non-saturation boundary).

Therefore, using the short-channel property of a MOSFET to control the delay will result i:1 two different delay regions, one region where a MOSFET is in linear operation and another whe $e_{2}$ it is in saturation mode. In the linear region, the delay rate of change is definitely higher at the boundary of the saturation region. 
Since the frequency of oscillation for a ring oscillator is a function of the delay, its voltage-to-frequency (V/F) characteristic can be expected to have the same transfer characteristics as the delay element. Figure 4.6 shows a simplified version of the proposed three-stage-ring oscillator employing a single-stage-inverter delay cell.

The modified three-stage ring oscillator consists of a delay cell with its frequency control input rearranged, and two inverters connected to form a closed loop ring. The circuit results in the least rumber of components required for a functional relaxation oscillator.

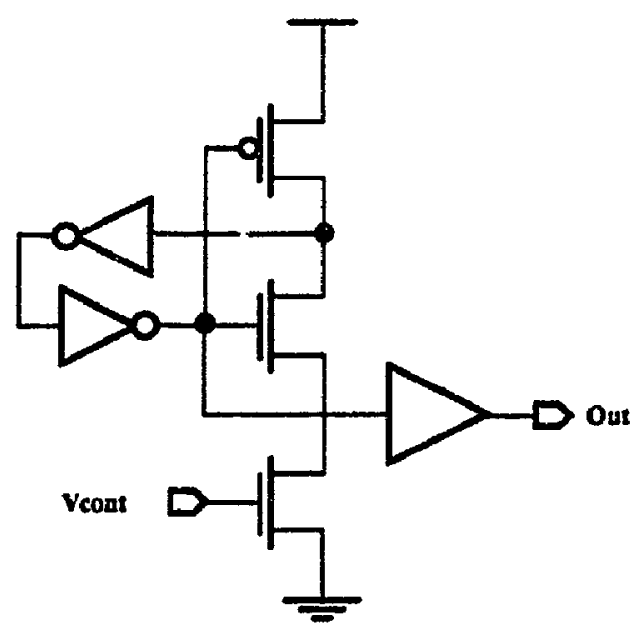

Figure 4.6 VCOI simplified three-st -ge modified ring oscillator.

\subsubsection{Modified D-latch ring oscillator}

Using the self oscillating behavior inherent in the proposed version of the D-latch circuit [5] (see Figure 4.7), a high frequency oscillator can be realized. The arrangement needed to construct this type of oscillator is shown in Figure 4.8, where a cross-coupled D-latch is combined with two loop inverter rings. $M p_{1}$ and $M p_{2}$ are used in this configuration to replace two complete inverters esulting in a pseudo-three-stage ring oscillator structure. 


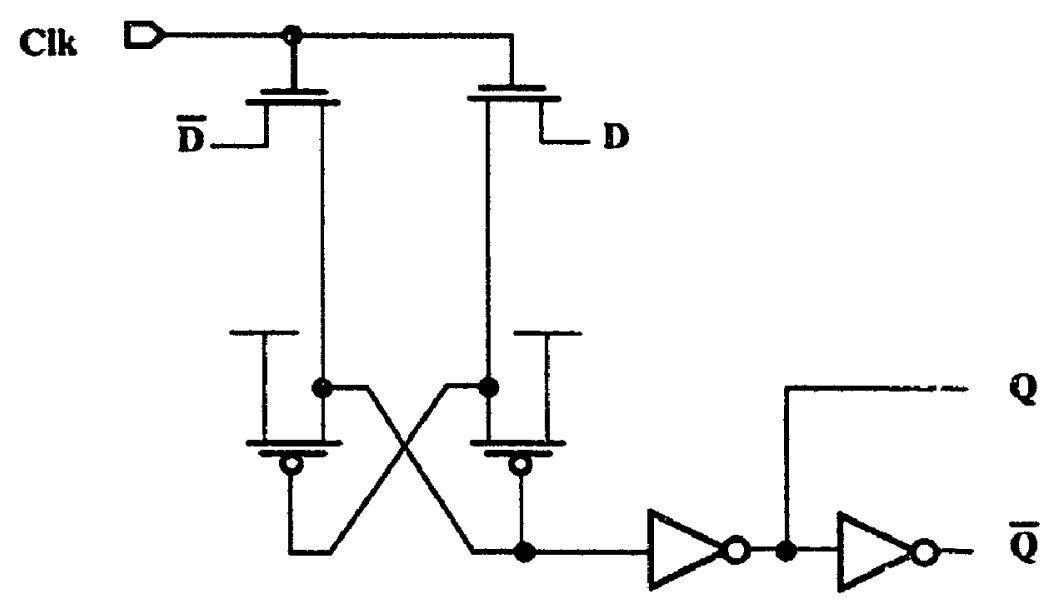

Figure 4.7 Proposed new D-latch for vCO2.

Since the proposed D-latch is a sequential circuit, when the Clk input (shown in the schematic as $V_{\text {cont }}$ ) is held high (logic 1) state transition occurs, enabling the rings to start oscillation.

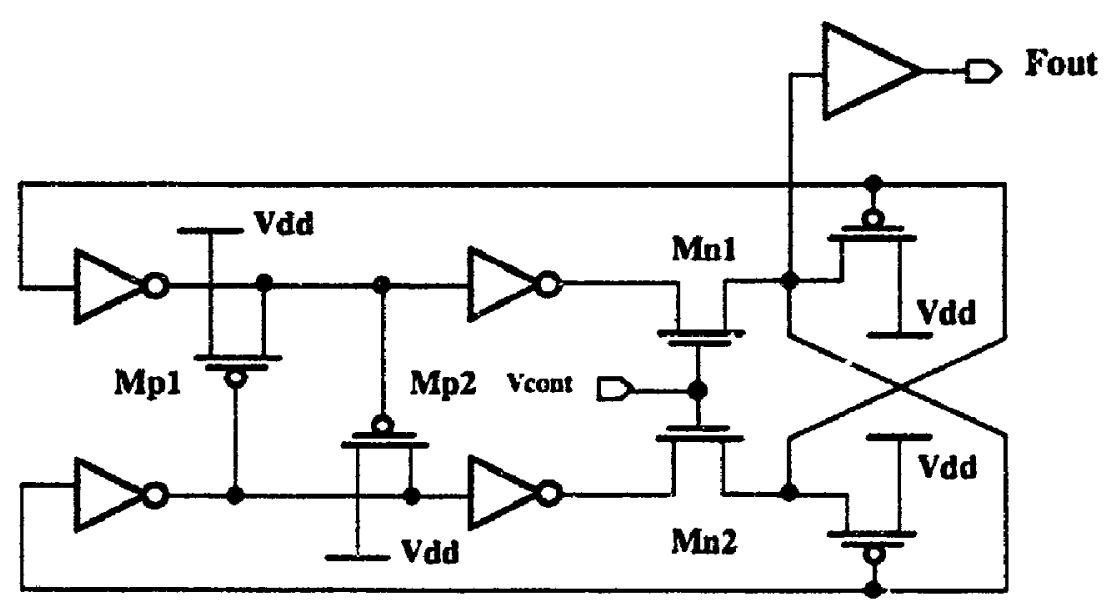

Figure 4.8 VCO2 modified D-latch ring oscillator.

The de level of Clk should be held highter than the threshold 2.5 volts in order to start the oscillation. A further increase in the dc level of the Clk results in the proportional 
decrease in resistance of $\mathrm{Mn}_{1}$ and $\mathrm{Mn}_{2}$, and consequently, the transition time in the latch is reduced. As a result, the frequency of oscillation can be varied with changes in $V_{\text {cont }}$.

The modified D-latch oscillator allows the oscillator to operate at high speeds while maintaining good noise performance, since the configuration is a cross-coupled ring structure (differential-like structure, see Section 3.2.6.2).

\subsubsection{Fully-differential ring oscillator}

The most popular configuration for a high-frequency VCO to date is the emittercoupled multivibrator. High frequencies up to several gigaherts. have been reported using a special bipolar process [56]. Derivations of this type of oscillator, which have also appeared recently, show that low phase noise can be accomplished by using specialized compensation techniques $[3,42,48]$.

As introduced in Chapter 3, standard emitter-coupled multivibrator designs have conflicting design rules which dictate that the oscillator consume high power in order to reduce phase noise. This standard approach has been modified to accommodate MOS implementations [52, 75]. NMOS versions of this emitter-coupled-like oscillator, fabricated in a $0.75-\mu \mathrm{m}-\mathrm{NMOS}$ process, show the use of ground timing capacitance exploiting parasitics only. A wide tuning range and maximum oscillating frequency of $1 \mathrm{GHz}$ were achieved. A CMOS version of this architecture was explored and suggested but no details relating to the analysis nor implementation were given. Figure 4.9 shows the proposed structure for a CMOS implementation.

The oscillator was constructed using a constant current-charge-discharge scheme, as explained in Chapter 3, and latching operations which alternate the state of two current controlled inverters were achieved by the use of double flip-flops. 


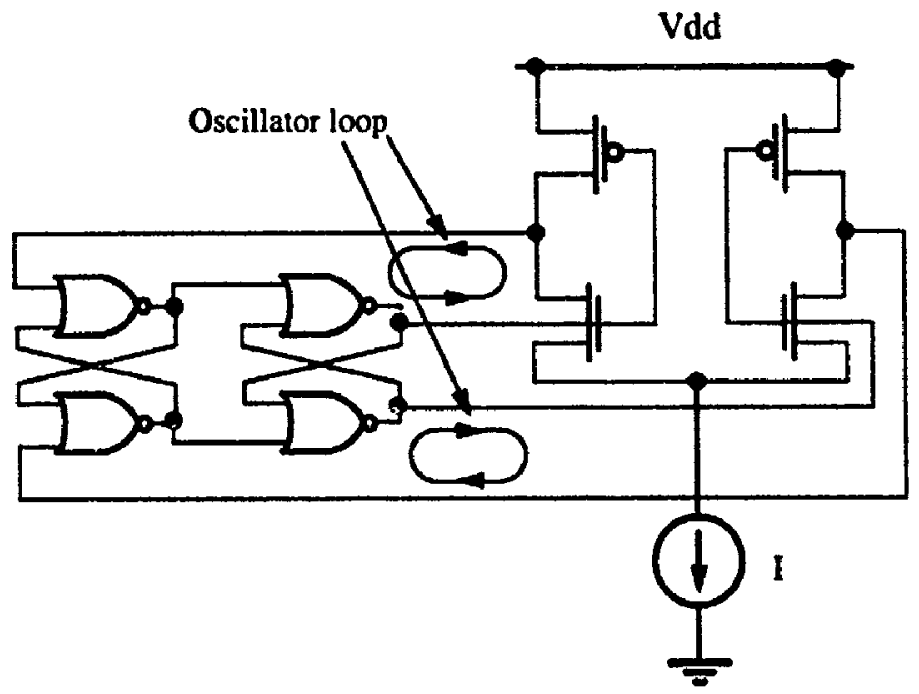

Figure 4.9 CMOS implementation of emitter-coupled structure using double flip-flops.

Since NOR flip-flops are based on the operation of inverter logic, the architecture can be further broken down to a simple inverter-level analysis by looking at the structure as a two-loop differential ring oscillator, as shown in Figure 4.10. The rings are formed by common three-stage ring oscillators.

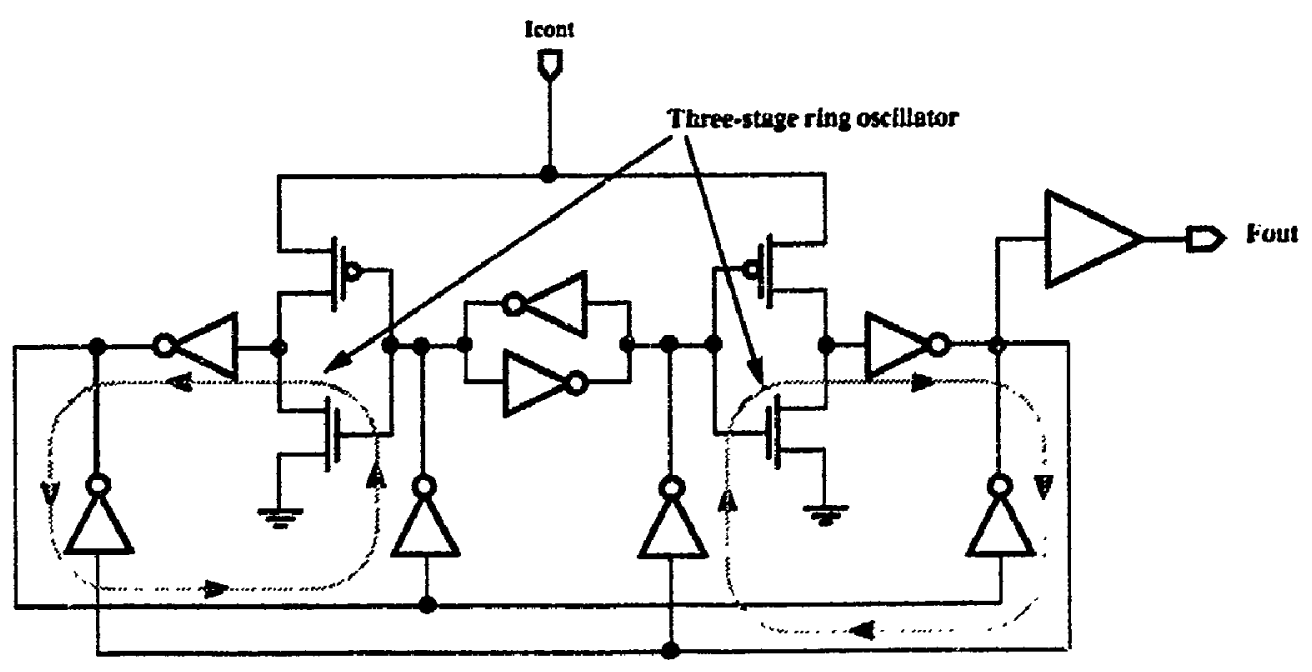

Figure 4.10 vCO3 fully differential latching comparator oscillator. 
Althoigh this VCO design is based on a proposed architecture found in the literature [52], significant work and optimization are required to achieve the desired low noise and maximum oscillating frequency. A simplified schematic of the complete VCO is shown in Figure 4.10.

To appreciate the significance of the differential ring architecture in this VCO structure, Figure 4.10 has been modified to Figure 4.11 , thowing the rearranged architecture. This explicitly shows how the two inherent differential structures and their regenerative elements (circled) are formed using NOR flip-flops in latching circuits.

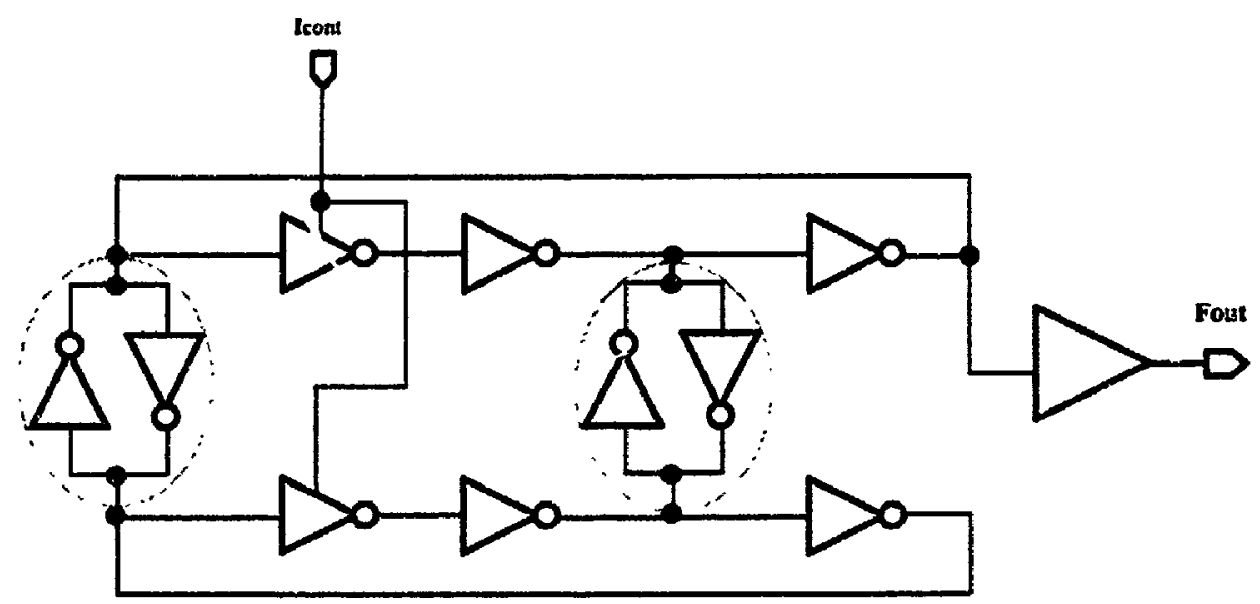

Figure 4.11 VCO3 shown with its inherent regenerative circuits.

The differential inverter rings are now formed using two cross-coupled 5-stage ring oscillators via regenerative inverter latches. These regenerative circuits also help to reduce the switching inconsistency of the waveform across the parasitic capacitance of the parasitic-effect-based relaxation oscillator (see Section 3.2.6.1). 


\subsection{VCO implementation (CUPLL)}

This section describes the implementation of the proposed VCOs presented in the previous section. The VCO circuits are optimized both sithematically and in layout prior to fabrication. Various simulations were performed for all circuits in order to characterize operation and functionality as well as to maximize the oscillating frequency. Layout consideration issues related to noise and speed improvements are also presented.

\subsubsection{Circuit optimization}

Simulation of the preliminary design of all three VCOs was carried out at the schematic level. Time domain analysis was performed in order to find the maximum possible oscillating frequency. Simulations show that optimized circuits and device sizing have to be made through a series of trials due to the dynamic interaction between the loop ring components, as explained in Chapter 3. However, a design guidelines that can be used for inverter ring oscillators are enumerated below:

\section{Equivalent load concept [59]}

For fast switching speed, large currents and a small load are required to insure small charging and discharging time constants. These contrasting requirements lead to a design problem.

To increase current flow, one must increase the (W/L) ratio, which in turn increases the transistor capacitance. Increasing the aspect ratios in a CMOS circuit leads to larger values of $\mathrm{C}_{\mathrm{in}}$ and $\mathrm{C}_{\mathrm{out}}$, affecting the performance of the entire oscillator. Therefore, an attempt to optimize the circuit must be made in the individual inverter stage with respect to its nearest neighbors.

A rule of thumb that can be used is that all inverters that drive more than two loads should be sized larger. Otherwise, the smallest possible inverter should be used. 
2 Eliminating the offending nodes that slow down the overall speed

By analyzing the current and voltage waveforms of all circuit nodes, performance optimization can be achieved. In the event of slow rising or falling waveforms, circuits should be corrected by either changing the device size or rearranging the layout configuration.

3 Sizing all inverters to have a $50 \%$ duty cycle so as to obtain the lowest possible phase noise [35].

This is achieved by balancing the $n$ and $p$ MOS aspect ratios so that the load line of the dc transfer characteristic exhibits unity gain at the input voltage of 2.5 volts.

The design guidelines listed above were applied in an attempt to meet the design goal of increasing the maximum oscillating frequency of all proposed VCO configurations, VCO1, VCO2 and $\mathrm{VCO} 3$ (or iCú). An initial temperature variation simulation was also performed. The following results were obtained without the inclusion of parasitics (Table 4.2).

\begin{tabular}{|c||c|c|c|}
\hline Parameters & VCO1 & VCO2 & VCO3 \\
\hline \hline $\begin{array}{c}\text { Mits. Osc. Freq } \\
\text { (MHz) }\end{array}$ & 820 & 408 & 200 \\
\hline $\begin{array}{c}\text { Max. Osc. Freq } \\
\text { (MHz) }\end{array}$ & 1,141 & 928 & 825 \\
\hline $\begin{array}{c}\text { Power Consumption } \\
\text { at Max. Freq. (mW) }\end{array}$ & 4.5 & 10 & 13.5 \\
\hline
\end{tabular}

Table 4.2 VCO schematic level simulated results. 


\subsubsection{Layout optimization}

In this section, layout related design ccussiderations associated with speed improvement, noise and circuit partitioning are presented.

The circuits were laid out using CMOS4S, a 1.2um double-poly double-metal $\mathrm{N}$ well CMOS tecinology (see Appendix A). The CADENCE-EDGE design environment was used as the design interface.

One of the crucial factors in achieving top speed in a CMOS design is the optimization of the circuit layout. For circuits to operate at frequencies close to those of their pre-layout simulation results, standard cells had to be avoided. Instead, custom designs were created for each VCO configuration, incorporating optimization techniques previously described.

In order to minimize the parasitic capacitances, the core VCO layouts were made as compact as possible, and all interconnections were made using metal layers. Minimal metal lengths were used by using diagonal or $45^{\circ}$ inetal lines wherever possible. All transistors employed minimum-design features except for the transistor widths. The polysilicon layer was used exclusively for transistor gates, and all transistors had a $1.2 \mu \mathrm{m}$ gate length. All $\mathrm{p}$ and $\mathrm{n}$ transistors were laid out symmetrically to achieve a $50 \%$ duty cycle ou.put, thereby decreasing output harmonics and jitter.

Transistor sizing was carefully optimized using a series of simulations between HSPICE extracted layout simulations and laycut fine adjustments until no further improvement in the maximum oscillating frequency was achieved. Actual transistor sizes are provided in Appendix B.

Power and ground lines were laid out as $20 \mu \mathrm{m}$ wide metal lines. Star-ground techniques were employed to prevent ground loops and power rail ripples, resulting in lower current spikes and ground bounce which could lead to increased VCO phase noise. 
Finally, buried ground rings were placed around each VCO circuit to reduce noise coupling from adjacent circuits. On-chip capacitors were placed between Vdc' nd ground lines, on unused space, to decouple high frequency noise on the power supply.

Due to very high-frequency operation, standard output pads for the VCOs had to be redesigned. The standard digital pads provided in the CMOS4S standard cell library were not designed for clock frequencies higher than $59 \mathrm{MHz}$. Simulation confirmed that using digital pads resulted in severe VCO signal attenuation (more than $80 \%$ of the signal). Analog pads were therefore chosen. Although the output waveforms still suffered from huge pad capacitances, their amplitudes were larger than the digital pad implementation.

The layout of VCO1, VCO2, and VCO3 circuits including buffer circuits have active areas of $84 \mu \mathrm{m}$ X $50 \mu \mathrm{m}, 142 \mu \mathrm{m}$ X $58 \mu \mathrm{m}$, an: $127 \mu \mathrm{m}$ X $76 \mu \mathrm{m}$ respectively.

\subsection{Simulation results}

This section presents results based upon three types of simulation technique. The first approach incorporates a SPICE time domain simulation using parasitic component values extracted after the layout was generated.

The second simulation technique characterizes the VCO performance in the frequency domain as described in Section 4.1. The goal of this type of simulation is to supplement the time domain result and aid in the design of the parasitic-capacitor-based relaxation oscillator, providing more control in VCO behavior. This also valid ates the accuracy of the proposed model.

The third simulation approach demonstrates the capability for noise rejection, referred as a qualit; factor, by using a simulated phase noise evaluation method. The results from this simulation provide a verification for the second simulation : nethod. This method: requires a knowledge of both discrete time domain analysis and FFT spectrum analysis. 


\subsubsection{Post layout extraction simulation}

Various post-layout extraction simulation!; were performed to characterize the VCO. Figure 4.12 shows the simulated VCO waveforms using a swept dc input signal (dashed line). $\mathrm{VCO}^{\prime}$ (ICO) was driven by a swept current rather than the swept voltage used for Ṽu1 and VCO2. The maximum sweep voltage for VCO1 and VCO2 was limited to 5 volts in order to minimize latch-up problems in chip testing. All simulations were performed at nominal tenxperature of $25^{\circ} \mathrm{C}$ and 5 volt power supply conditions.

The top graph of Figure 4.12 shows the simulated waveforms of VCO1. The solid line is the waveform from the core circuit prior to the buffer input, and the dotted line is the waveform on the outpur pad. A large dc offset can be observed on the output pad as frequencies rise. There are also significant levels of attenuation at the highest frequencies. Simulation results show that the frequency of operation ranges from $328 \mathrm{MHz}$ to 776 MHz.

The middle waveform shows the simulation results obtained from VCO2. The VCO starts oscillating when input voltage $\mathrm{V}_{\text {cont }}$ is greater than 2.7 volts, and the oscillating frequency increases with increases in $V_{\text {cont }}$, resulting in positive transfer characteristic. Simulated results demonstrate that $\mathrm{VCO} 2$ has an operating frequency range from 236.27 $\mathrm{MHz}$ to $610.69 \mathrm{MHz}$.

VCO3 simulations are illustrated in the bottom graph of Figure 4.12. The oscillating frequency is a function of current $I_{\text {cont }}$. The operating frequencies were measured at a 127.88 MHz minimum for an $I_{c o n t}$ of $20 \mu \mathrm{A}$, and a maximum frequency of $540.10 \mathrm{MHz}$ for an $I_{\text {cont }}$ of $700 \mu \mathrm{A}$.

The transfer characteristic (V/F and $\mathrm{V} / \mathrm{F}$ curves) of the three VCOs are plotted in Figure 4.13. 

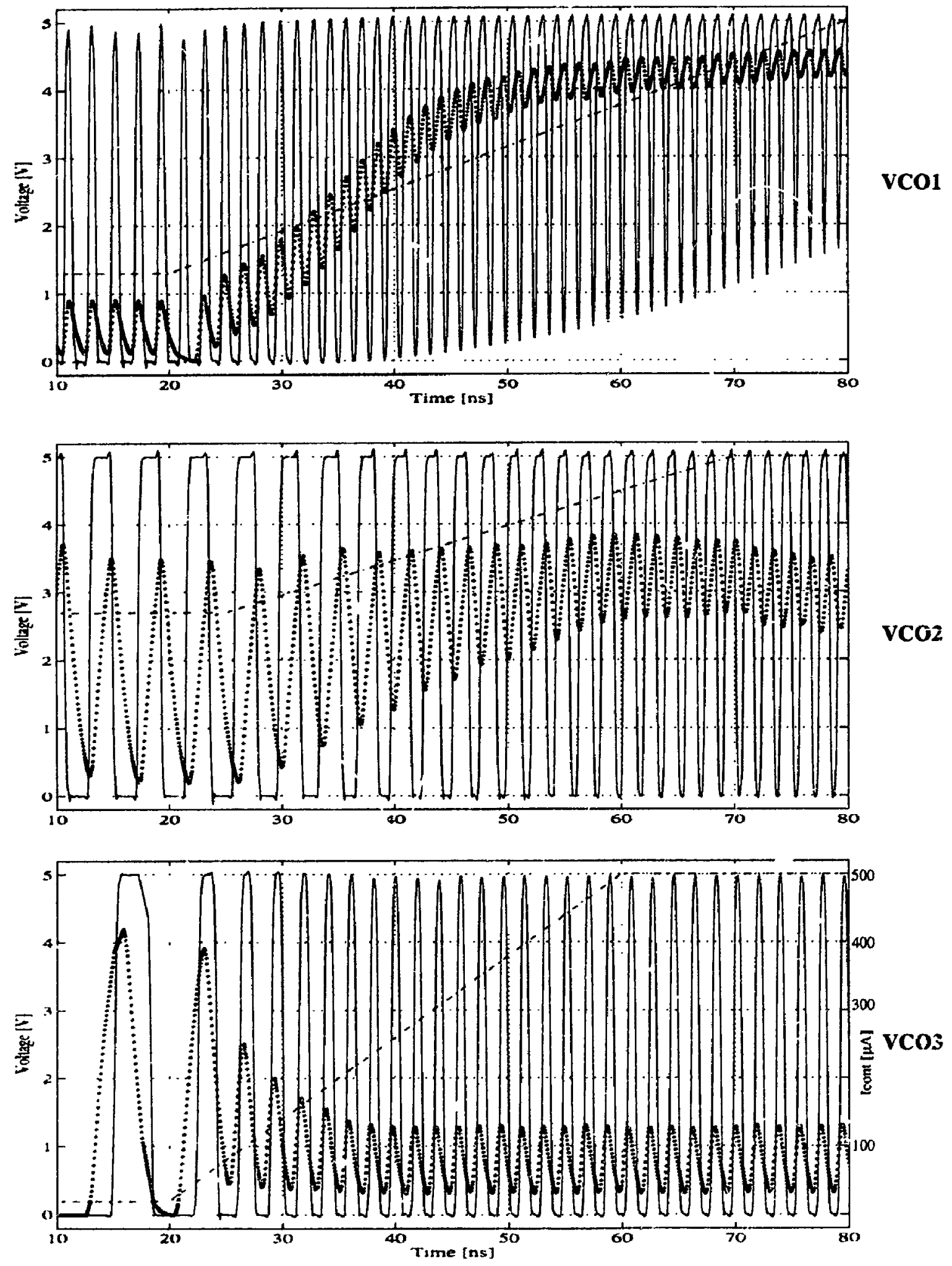

Figure 4.12 Simulated VCO outputs, dotied line are the signals probed at the output PADs. 


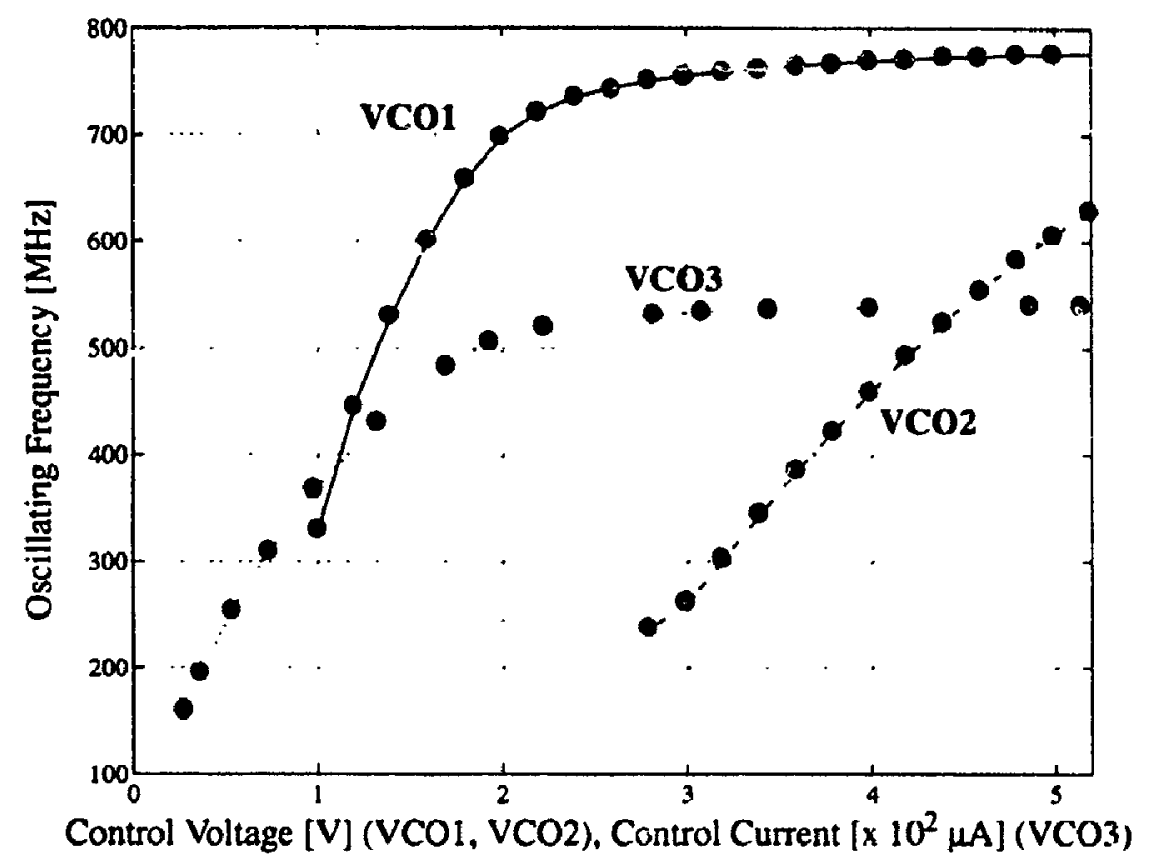

Figure 4.13 VCO transfer chara :"aristics.

From Figure 4.13, VCO1 has two values for its tuning constant $\mathrm{K}_{\mathrm{vco}}$. The first is 254 $\mathrm{MHz} / \mathrm{V}$ for $\mathrm{V}_{\text {cont }}$ less than 2.2 volts, and at $14 \mathrm{MHz} / \mathrm{V}$ for $\mathrm{V}_{\text {cont }}$ above 2.2 volts. The first region is a result of the MOS devices operating in the linear region, while the second region resuts from operation in the saturation mode. Both agree with the predicted characteristics of the proposed delayed cell. It can be seen that even though the operation of MOS devices, biased by $\mathrm{V}_{\mathrm{gs}}$, approaches the saturation region, current still increases monotonically with respect to the $\mathrm{V}_{\mathrm{gs}}$. This is due to the short-channel effect described previously.

In the application of a PLL synthesizer, a high VCO tuning constant $\mathrm{K}_{\mathrm{vco}}$ often causes a PI L to lose lock [11]. Therefore the use of the proposed ring oscillator should utilize the $\mathrm{V} / \mathrm{F}$ characteristic at the saturation region where the $\mathrm{VCO}$ produces a smaller $\mathrm{K}_{\mathrm{vco}}$. 
VCO2 demonstrates a linear V/F characteristic with a $\mathrm{K}_{\mathrm{vco}}$ of around $175 \mathrm{MHz} / \mathrm{V}$ across the entire range of control input.

VCO3, a current controlled oscillator, results in a VCO gain which is expressed in $\mathrm{MHz} / \mu \mathrm{A}$. Its transfer characte.istic curve is very close to VCO1. The early $\mathrm{K}_{\mathrm{vco}}$ is rather high at $227.81 \mathrm{MHz} / \mathrm{V}$ (if $100 \mu \mathrm{A}$ is normalized to 1 volt) and rapidly decreases to 10.41 $\mathrm{MHz} / \mathrm{V}$, similar to that obtained for VCO1.

The VCOs were simulated to examine their operating points as a function of temperature variations. Figure 4.14 shows the VCO characteristic curves for variations in temperature from $0^{\circ} \mathrm{C}$ to $80^{\circ} \mathrm{C}$.

Simulation of the VCO maximum oscillating frequencies as a function of power supply variation was performed. As well, power dissipation at various oscillating frequencies was also investigated. Figure 4.15.a shows the simulated res"lts when VCOs are operated with varying Vdds.

Since all three VCOs have been laid out together with their buffer circuits for maximim compaction, the power consumptions shown in Figure 4.15.b also include the power dissipated by buffer circuits. 


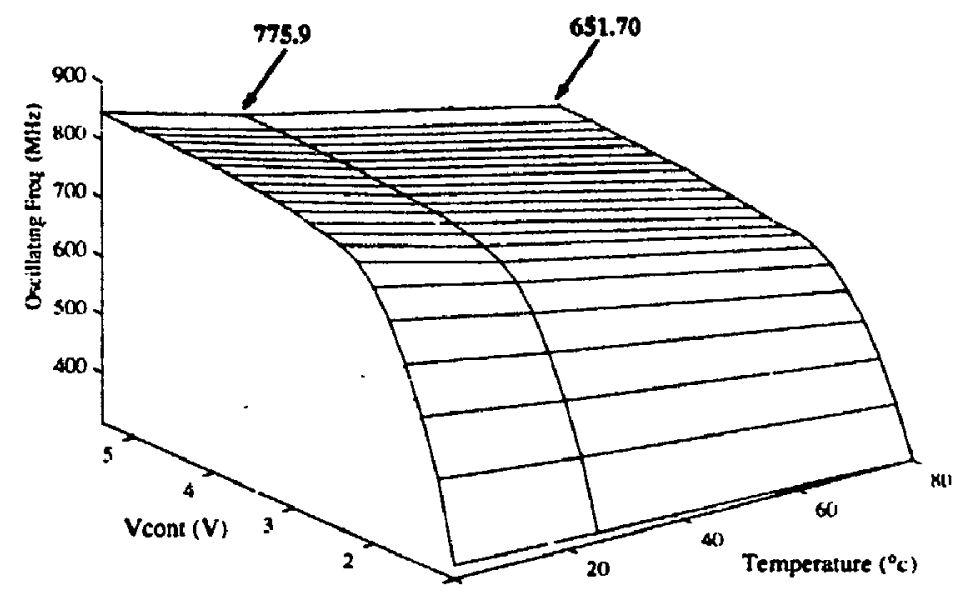

VCO1

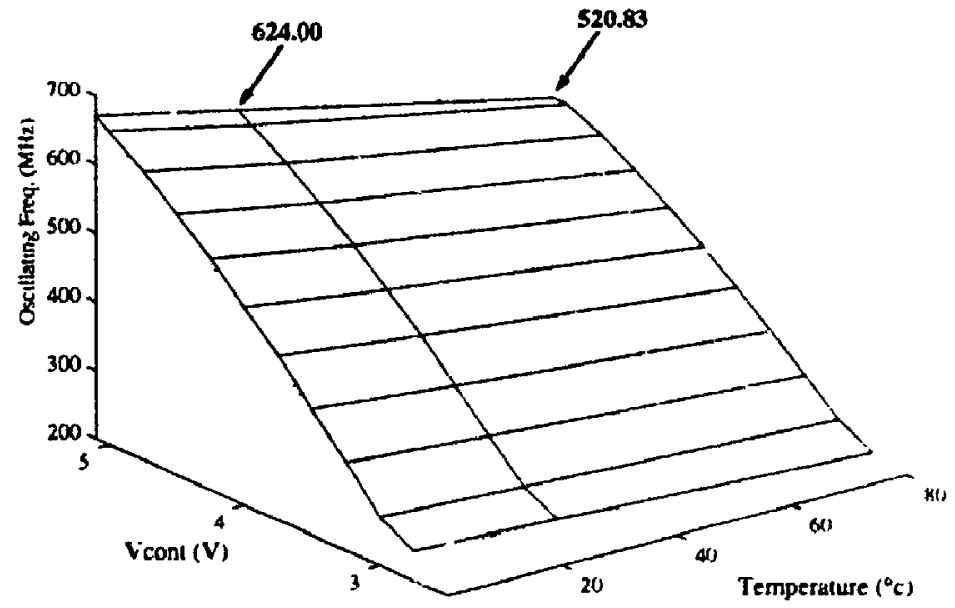

VCO2

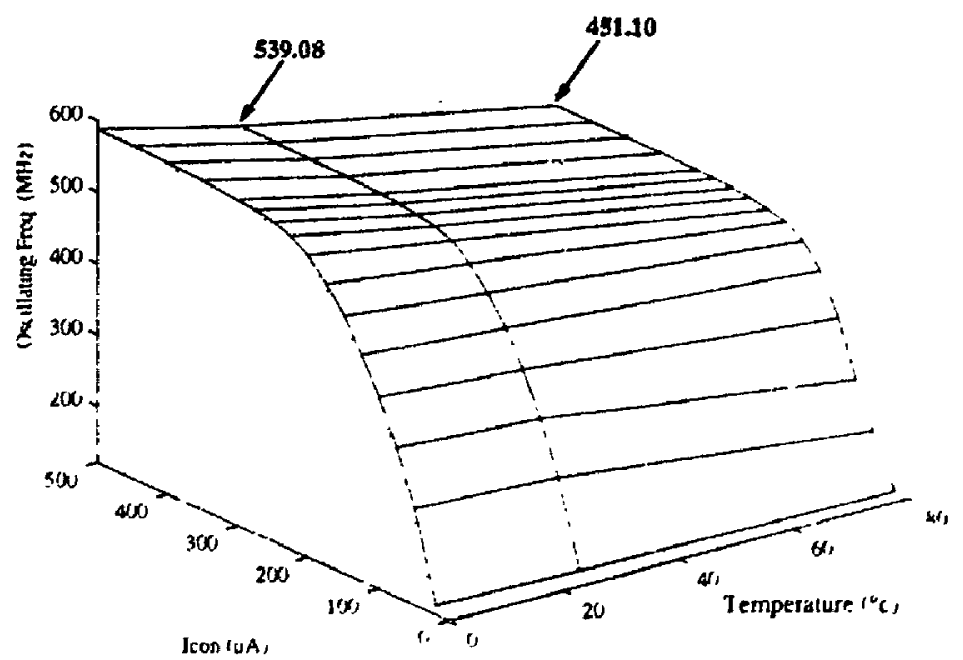

$\mathrm{VCO}$

Figure 4.14 Effects of temperature variation on the VCO uperating point. 


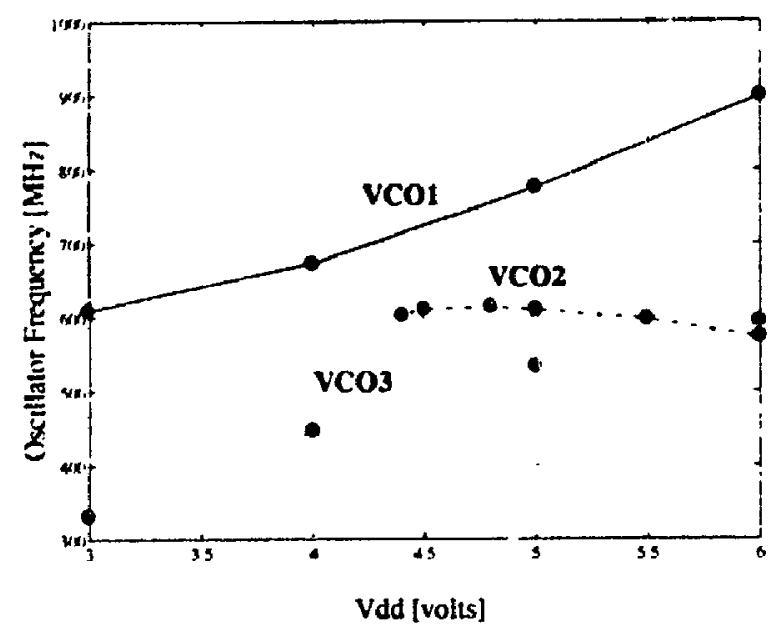

(a)

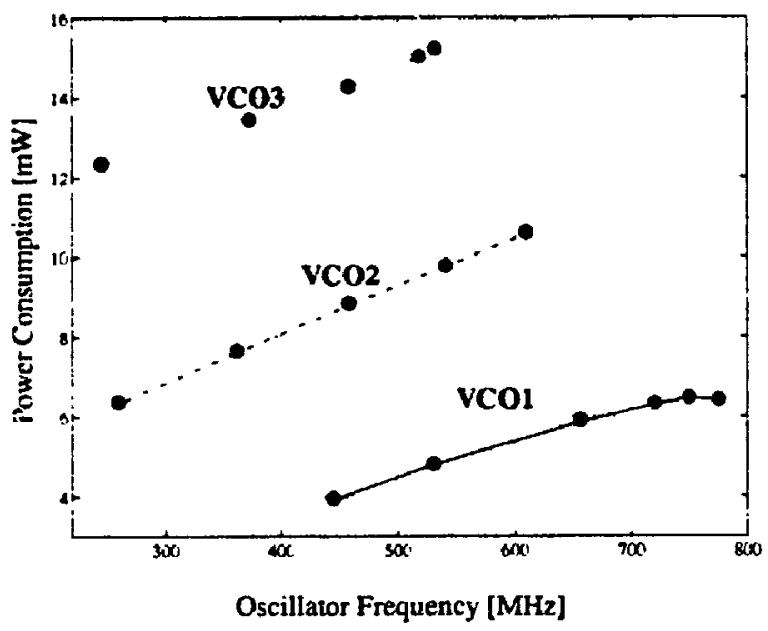

(b)

Figure 4.15 (a) Oscillator's maximum frequency versus supply voltages and (b) Power consumption.

\subsubsection{Simulation for validation of the proposed ac MOS model}

In order to validate the functionality and accuracy of the proposed morel, several si. : 'ations were performed using injected noise (see Section 4.1 ) and SPICE ac analysis.

To confirm the close correlation between the proposed model results and SPICE transient analysis, the developed model consisting of parameters shown in Figure 4.3 was verified with all parameters obtained from post layout extraction. This includes parasitics generated by layout configuration and the devices used in each VCO circuit. In this analysis VCOI was used as an experimental prototype for developing the correct ac model parameters.

The simulation procedure using SPICE level 3 can be described as follows. After the parameters in ear' $h$ MOS device were obtained from HSPICE transient analysis output $\left(C_{g g}\right.$, $\mathrm{C}_{\mathrm{gd}} \cdot \mathrm{C}_{\mathrm{gtot}}$ and $\mathrm{g}_{\mathrm{m}}$, they were recalculated and used in the developed ac MOS model. The MOS models are then represented by subcircuits and used to replace all MOS transistors at the same nodes (drain, gate, source and bulk), as in the origiral extracted layout. 
An ac analysis was then performed on the altered circuit netlist by adding an injected voltage controlled current source at node $X$ (small ac current source of $1 \times 10^{-.5}$ amperes) shown in Figure 4.16.
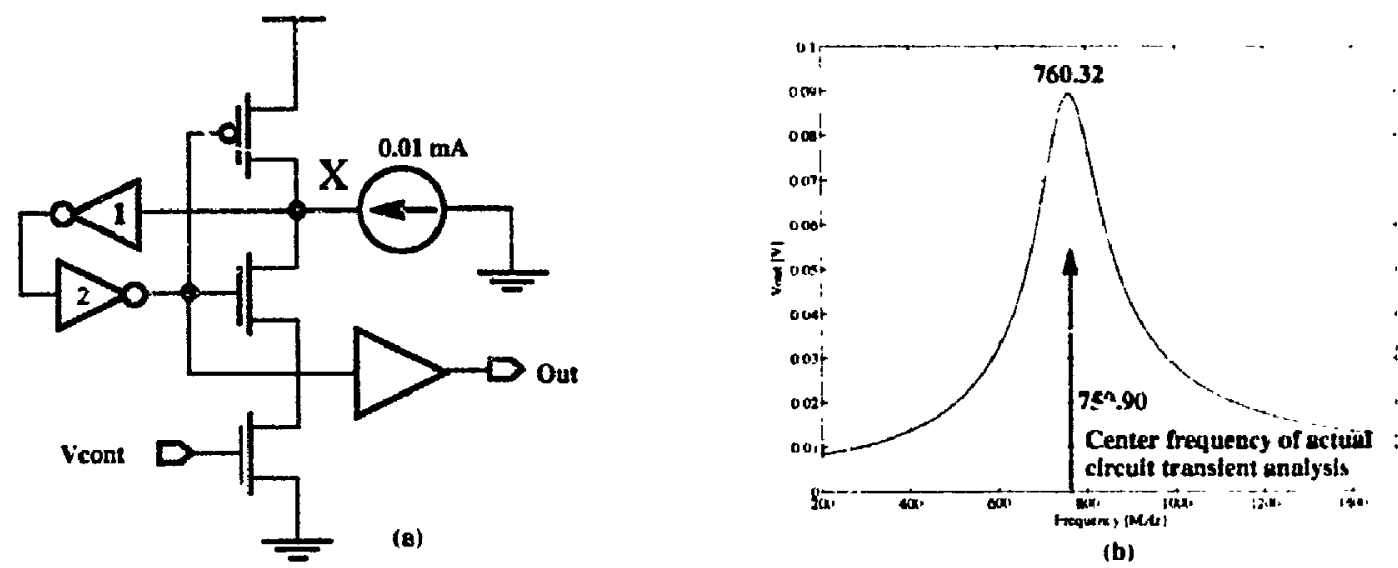

Figure 4.16 Simulation performed on developed ac MOS model using injected noise current source.

The simulated result of the linear ac model produced a peak frequency of 760.32 MHz. This deviates approximately $0.05 \%$ from the SPICE transient analysis result of the actual circuit.

The accuracy of the developed model has also been validated with simulations based upon different device sizes. The inverter 1 layout of VCO1 was changed, with both the $n$ and $p$ MOS six tirues larger than those of the original circuit. The extracted layout simulation was then performed and the transient analysis indicated an oscillating frequency of $673.40 \mathrm{MHz}$. The ratio of center frequencies between the inverter that was enlarged six times and the original circuit is $\mathbf{8 8 . 6 1 .}$

New device parameters extracted from HSPICE were then used to replace the ac MOS model parameters for ac simulation. The ac response of the ot illator produced a peak frequency of $701.45 \mathrm{MHz}$, as illustrated in Figure 4.17. 


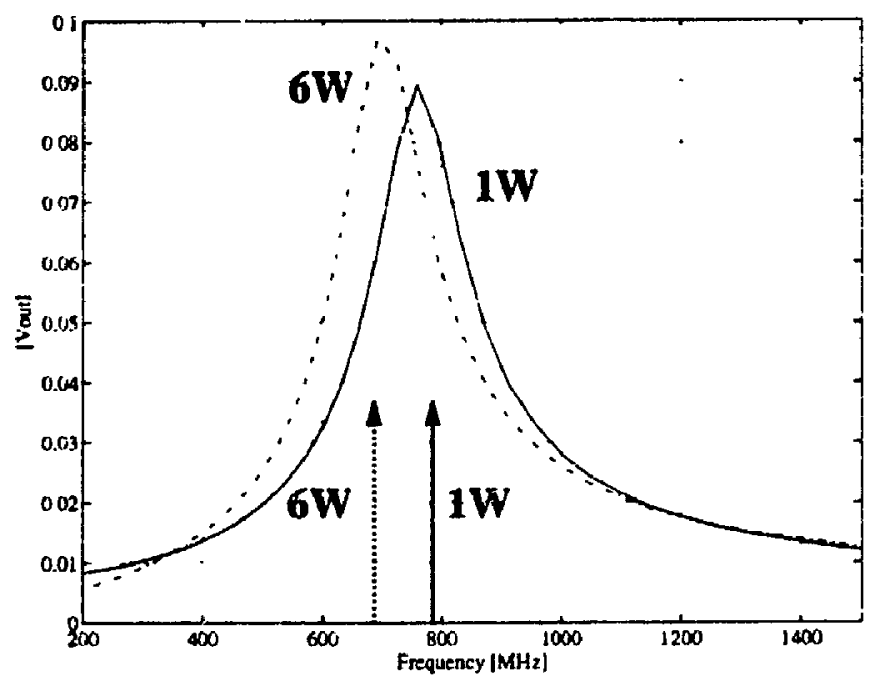

Figure 4.17 Frequency response of VCO1 to noise using developed ac model (arrows show the transient response of actual circuit).

In comparison to the center frequency ratio of the results obtained from the SPICE transient analysis, the results obtained using the developed model gave a ratio of center frequencies of 92.20. This indicates that the accuracy of the developed model is acceptable.

Further verification of the model was also made for $\mathrm{VCO} 2$ and VCO3. The results show that the equivalent ac MOS model is valid for all proposed CMOS VCO architectures, and can therefore successfully represent appropriate transfer characteristics of CMOS multivibrators.

Apart from this, the comparison of the frequency domain analyses of the three VCOs, operating under the same condition of added noise current, also demonstrates that each VCO shows distinction in noise susceptibility. Figure 4.18 illustrates the frequency response of the VCOs to injected noise. 


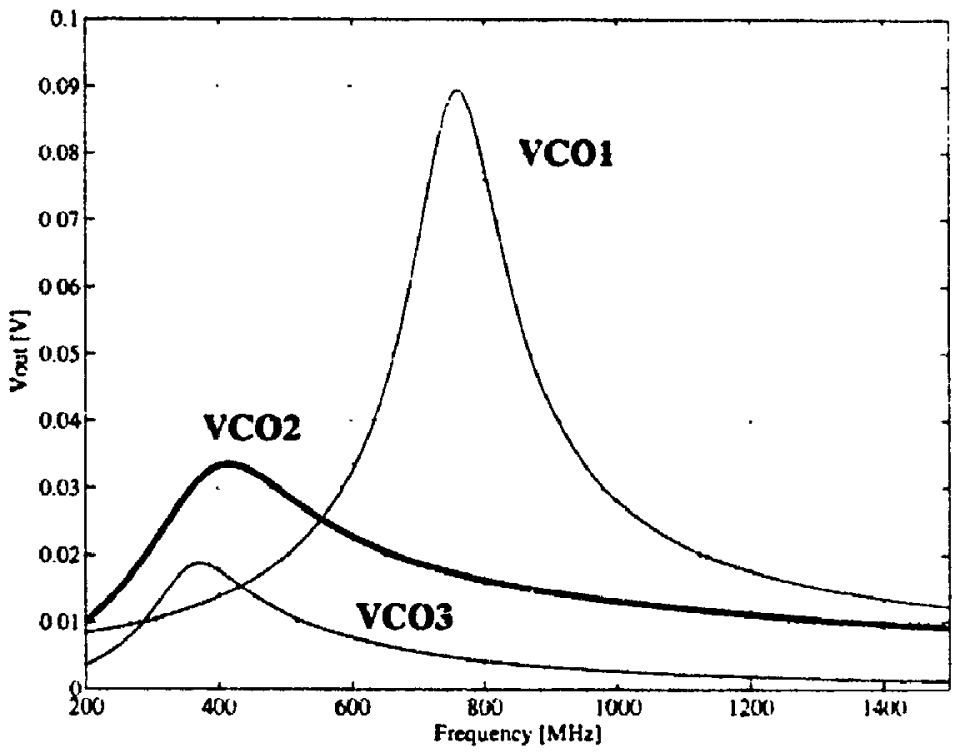

Figure 4.18 Frequency response of VCOs to noise using developed ac model.

\subsubsection{Phase noise simulation}

This section presents simulation results showing VCO spectral purity in the presence of power supply noise. The method is based on the fact that high-quality-factor (high Q) oscillators are insensitive to power supply and intrinsic noise regardless of its origin [68]. High-phase-noise oscillators therefore exhibit a higher degree of noise sensitivity.

The simulation technique is based upon the idea of noise referred input determination. Since the VCO architectures introduced in this work are implemented using CMOS, adding a noise source on the power rail provides the same result as injecting an equivalent noise sürce at the input, as shown in Figure 4.19. However. for efficient computation, only a single tone noise source injected on the power rail was chosen.

The phase noise evaluation by the power supply noise injection technique is intended to be used during the measurement stage, when the VCOs have been fabricated. Therefore, the simulated results can be verified with the test results. 


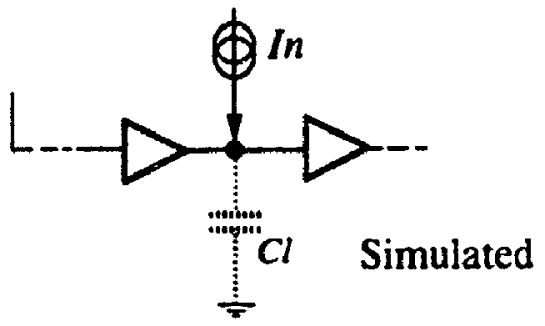

(b)

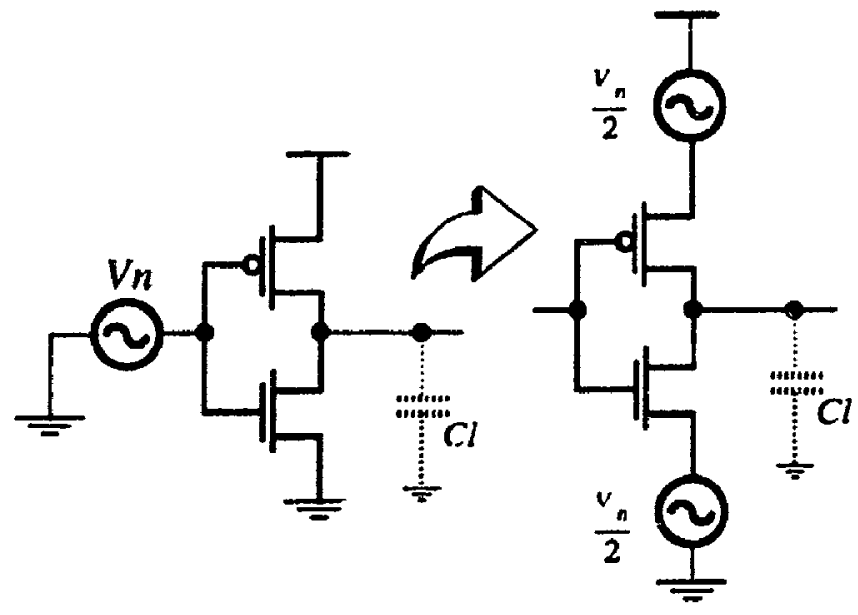

(a)
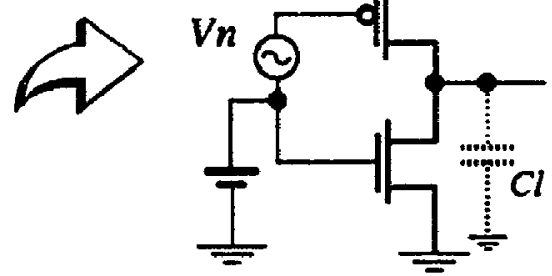

(d)

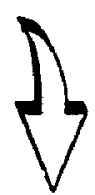

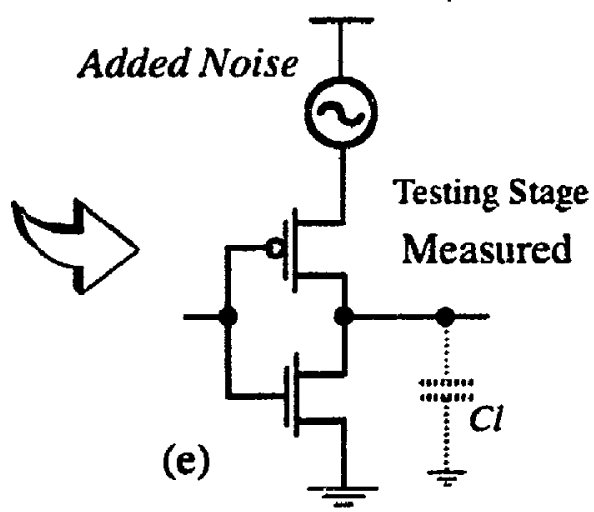

Figure 4.19 Equivalent circuits for a simulated injected noise current and its actual power supply noise superposition in the measurement stage.

Figure 4.20 shows the simulation technique for performing the phase noise analysis. The simulation procedure was carried out as follows. After the extracted layout netlist of each VCO was obtained (actual SPICE's MOS model), the modulated noise source, using a sinusoidal signal of $100 \mathrm{mV}, 1 \mathrm{MHz}$, was put in series with Vdd. Eldo [76], a mixed signal simulator, was used for transient analysis. The spectral analysis of the resulting oscillator output was subsequently performed by Eldo providing a power spectral density analysis (PSD) tool. 


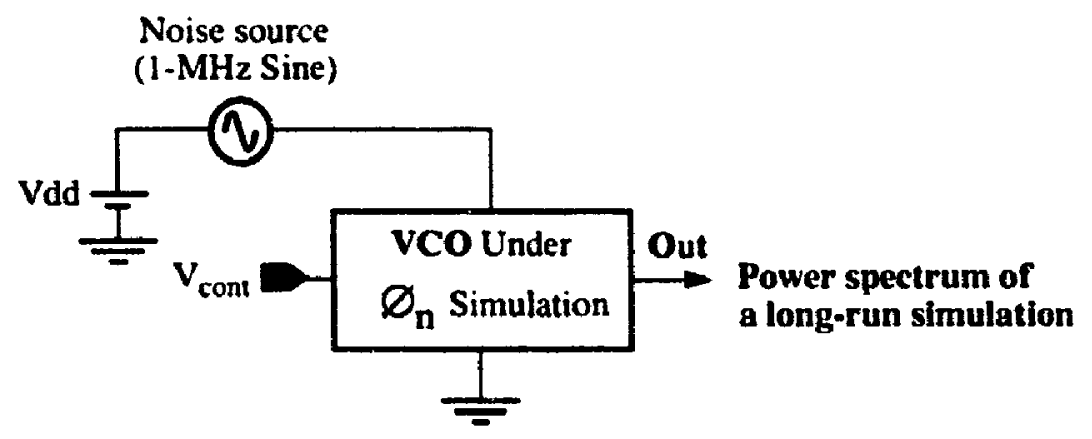

Figure 4.20 Simplified simulation setup for phase noise prediction technique.

In order to obtain reliable results, each VCO was chosen to operate under the same conditions. These include the choice of oscillating frequency, sampling frequency. simulation step size and number of points in the PSD analysis. However, since VCO3 has a maximum oscillating frequency of $520 \mathrm{MHz}$, an oscillating frequency for VCO3 of 500 $\mathrm{MHz}$ was chosen instead.

Figure 4.21 shows the results from the power spectral analysis. The left-hand-side plots are the PSDs of the VCOs when they are in the free running condition. The righthand-side plots show the PSD after having the noise superimposed on the power supply. It can be interpreted from the PSD analysis that each VCO has a different degree of sensitivity to power supply noise. The VCOl spectrum shows the widest area of modulation around its center frequency, while $\mathrm{VCO} 2$ exhibits less susceptibility to noise. Finally, VCO3 demonstrates the best noise rejection among the three VCOs. 

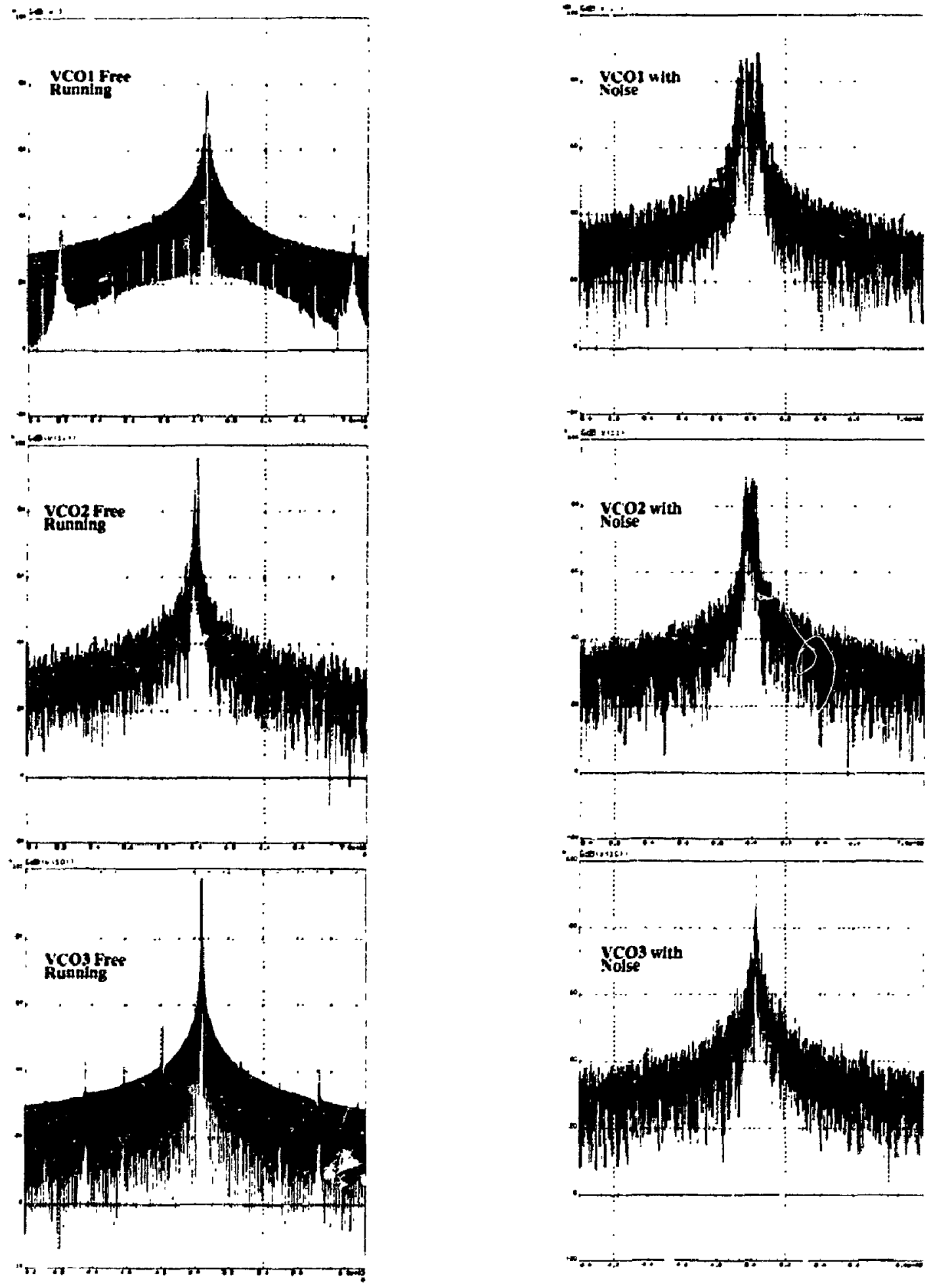

Figure 4.21 Simulated VCO spectra demonstrating phase noise correlations. 


\subsubsection{Phase-frequency detector (PFD) with no dead-zone}

The DIn without a dead zone [6] was part of the design for a CMOS PLL frequency synthesizer. This section reports on the simulation results which show its key performance in terms of linear phase detection range. The results indicate the practical linear operating range for a closed loop PLL synthesizer design.

The graphs shown in Figure 4.22 were obtained by simulating the PFD extracted layout in transient analysis und averaging the pulse train signal at the output of FrD using a low-pass filter. The average dc values were plotted for three input frequencies. The results show that the dead zone occurs at both ends of the lixtear range and increases proportionally with the input frenuency.

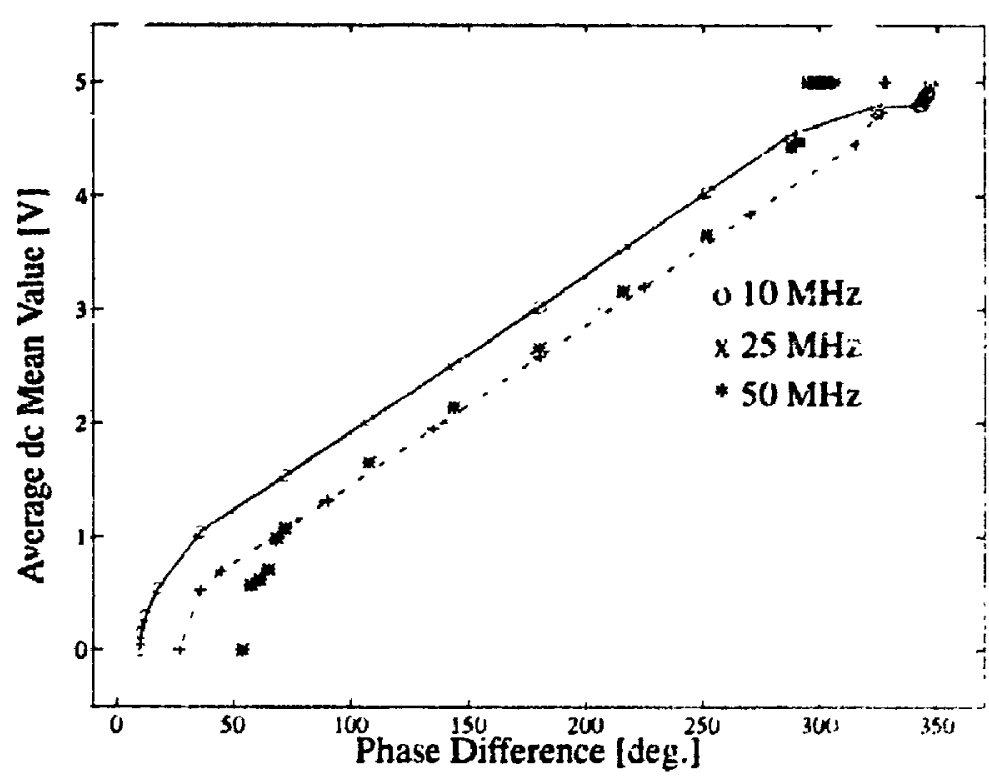

Figure 4.22 Simulated phase-frenuency detector characteristics, $\mathrm{f}_{\mathrm{ln}}=10 \mathrm{MHz}, 25 \mathrm{MHz}$ and $50 \mathrm{MHz}$.

The PFD operates linearly with input froquencies below $50 \mathrm{MHz}$. The linear phase detection range at $50 \mathrm{MHz}$ is 216 degrees and is cunsidered to be acceptable, since the PFD has a built-in frequency capability that assists the loop operation. 

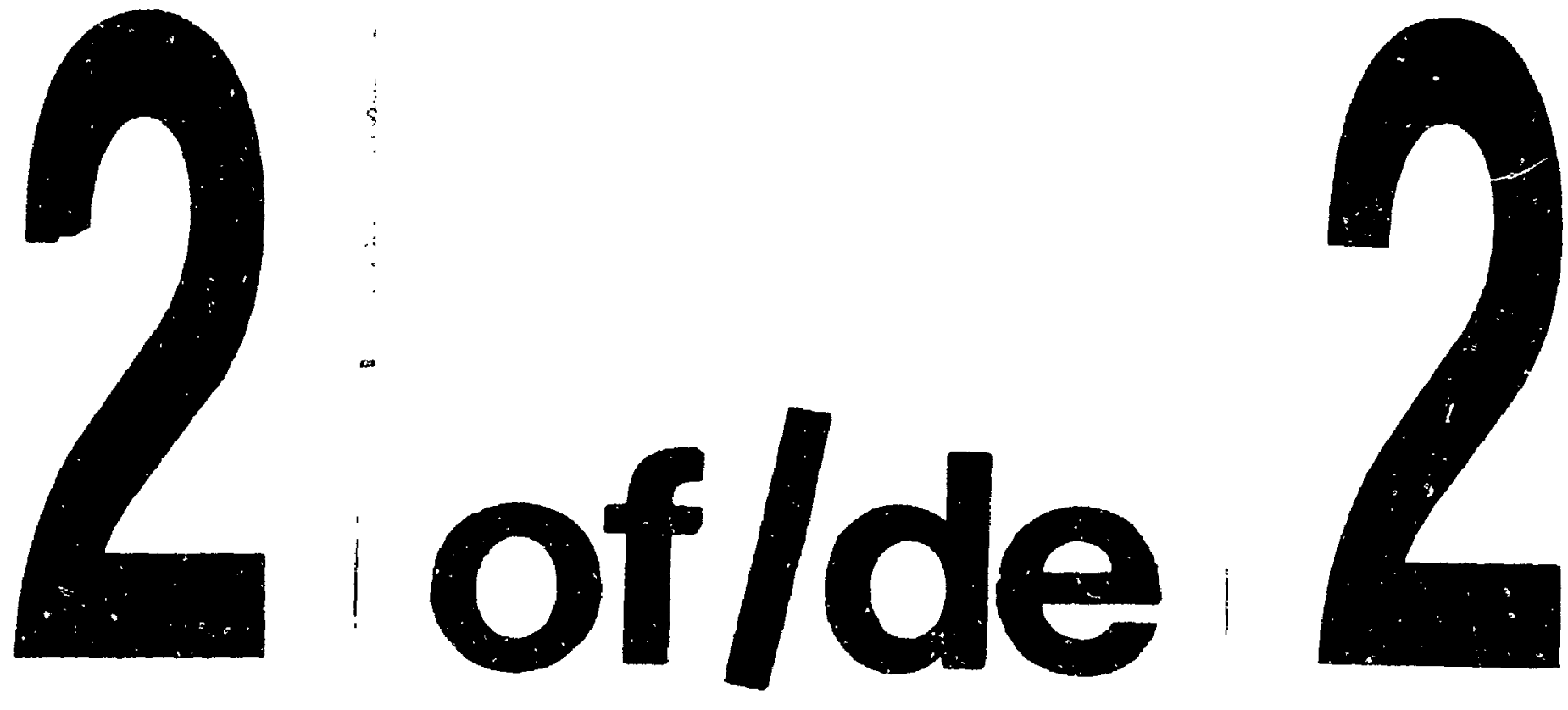

PM-1 31/2" $x 4^{\prime \prime}$ PHOTOGRAPHIC MICROCOPY TARGET NBS 1010a ANSI/ISO \#2 EQUIVALENT

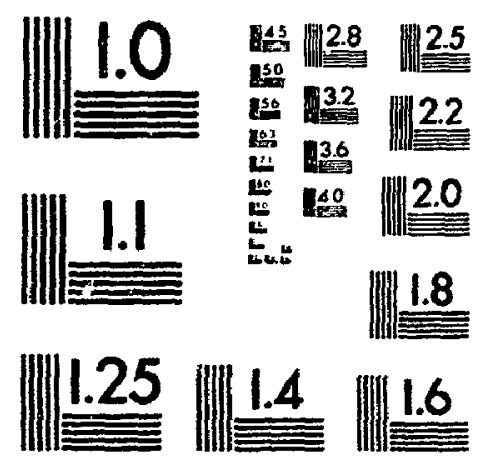

PRECISIONSM RESOLUTION TARGETS 


\subsection{Single-chip fractional- $N$ frequency synthesizer design}

With the CMOS PLL. components discussed itrdividually, a description and analysis of these components in a closed loop form (PLL) is provided in this section.

\subsubsection{PLL component implementation}

Owing to the satisfactory results from the first implementations of CUPLL, presented in the next chapter, a more complete CMOS PLL synthesizer (including a dualmodulus divider) was then implemented again in a CMOS process, 1.2 $\mu \mathrm{m}$-ga?e-length technology.

The synthesizer, CUPL2, consists of VCO1 (a modified 3-stage ring oscillator), a 15/16 dual-modulus divider [5], and a no-dead-zone phase-frequency detector based on the same design as the PFD in CUPLL.

All circuits were laid out initially with two configurations: (i) the VCOI embedded with the frequency divider configuration. With this design, the VCO signal would not be attenuated from the low-pass filtering effects of pad and package parasitics, since the VCO drives the frequency divider directly. This configuration also helps to reduce the total power consumption and noise. (ii) the components in stand alone configuration. This allows testing of both components individually and therefore the ability to pursue the test if the first configuration fails to function. The block diagram of the second configuration is shown in Figure 4.23.

The loop filter is chosen as an off-chip implementation for two reasons. First, the flexibility of loop gain and pole frequency selection, Second, implementation of the onchip loop filter requires a good understanding of other PLL component behaviors. Therefore performance evaluation, excluding the loop filter, has to be characterized prior to the implementation of an on-chip loop filter. 


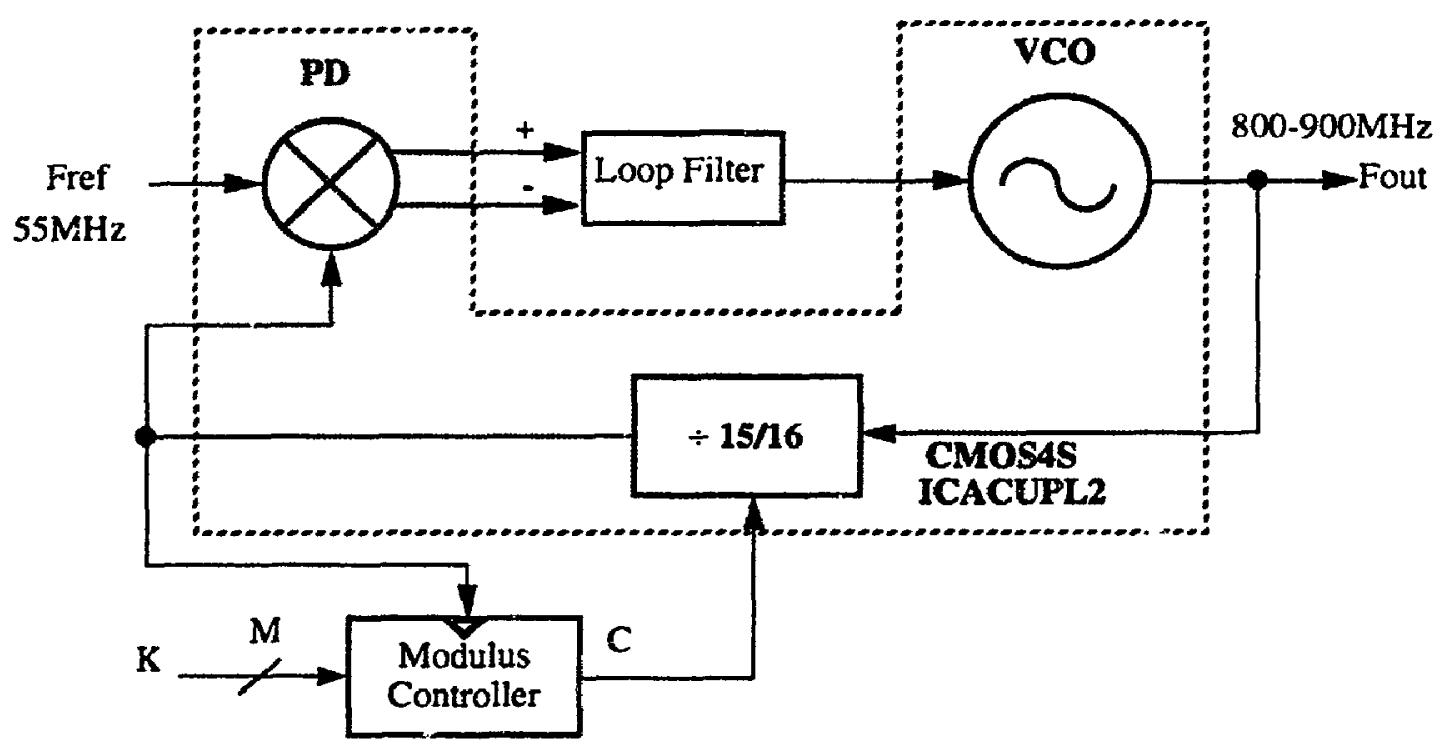

Figure 4.23 Single-chip inplementation of the CMOS frequency synthesizer, CUPL2.

Due to the complexity and to time constraints at the design stage, the simulation results for the complete fractional-n synthesizer were not performed. However, the performance results for full circuits will not differ considerably from those of the individual simulations presented by Foroudi [5]. The results of this implementation are therefore presented in the form of measured results which will be discussed in the next chapter.

\subsection{Summary}

Key design issues for implementation of a high-frequency low-noise CMOS oscillator have been discussed. VCOs based on the operation of parasitic timing elements, that accommodate a mobile radio single-chip frequency synthesizer, have been proposed and analyzed. It has been demonstrated that a high speed (GHz) CMOS VCO can be achieved with several design techniques including selection of an application specific VCO architecture, circuit simplification, and layout optimization. Design of three CMOS VCO structures with the outlined techniques were implemented in a $1.2 \mu \mathrm{m}$ process. 
A suitable ac MOS model was developed and its accuracy validated using the realized VCOs, resulting in a new tool that can be used to achieve more accurate simulations and therefore predict behavior prior to the selection of a custom VCO design in the future. A phase noise simulation method proposed in this work also supports the design of a CMOS oscillator to obtain insight in the inherent performance of the VCO structures. Finally, an all-CMOS synthesizer design has been introduced and fabricated which is believed to be the first CMOS synthesizer for mobile radio applications.

The next chapter discusses the test results and confirms the operation and functionality of the proposed design as well as verifies the viability of the simulation methods. 


\section{Chapter 5}

\section{Experimental Evaluation and \\ Test Results}

The integrated circuit with the three VCOs and the no-dead-zone phase-frequency detector, CUPLL, was first fabricated at Northern Telecom Electronics in a 1.2- $\mu \mathrm{m}$ double-polysilicon double-metal $\mathrm{N}$-well CMOS process (CMOS4S) through Canadian Microelectronics Corporation (CMC). Several tests were performed to characterize the performance and functionality of the fabricated chip. After a performance evaluation of CUPLL had been carried out, a new design, CUPL2, consisting of three major PLL. components, a VCO, a 15/16 dual-modulus prescaler and a fully differential phasefrequency detector with no dead zone, was laid out and resubmitted. Overall performance of the CUPL2 chip was measured and found satisfactory in its function. This chapter presents experimental setups, test procedures and measured results performed for both chips in the format shown below: 


\section{VCO design}

- Voltage-to-frequency transfer characteristic

- Oscillating frequency as a function of supply voltage

- Power consumption as a function of power supply voltage

- FM single sided band phase noise using phase noise measurement system

- Verification of phase noise prediction technique

Phase-frequency detector with no dead zone

- Phase detector characteristic

Closed Luop PLL frequency synthesizer

- Overall phase noise of synthesizer.

\subsection{CUPLL experimental setups}

In this section, test results for VCO and phase-frequency detector performance are reported. The tests are based on standard procedures for VCO and PFD performance evaluation. In industry the same kind of test may vary as a result of the definition set by an individual measurement system, however, the test setups here conform to most major VCO tests.

\subsubsection{CUPLL VCO test setup}

A simple test bed constructed from a printed circuit board (PCB) and other discrete components was used to provide power supply and VO interfacing for the three VCOs (see Figure 5.1). All measurements were performed on bonded IC's, packaged with a standard ceramic 68-pin PGA (Pin Grid Array) type. Two three-terminal adjustable voltage regulators, LM317s, supplied variable dc power ranging from $2.2 \mathrm{~V}$ to $6.0 \mathrm{~V}$ to the test board. One regulator output was used for the VCO power supply and the other for the frequency control input. Both voltage regulators' inputs were sourced with an 8-volt dc supply from an external power supply. Each VCO circuit under test had only four pins that connected to the test fixture. 


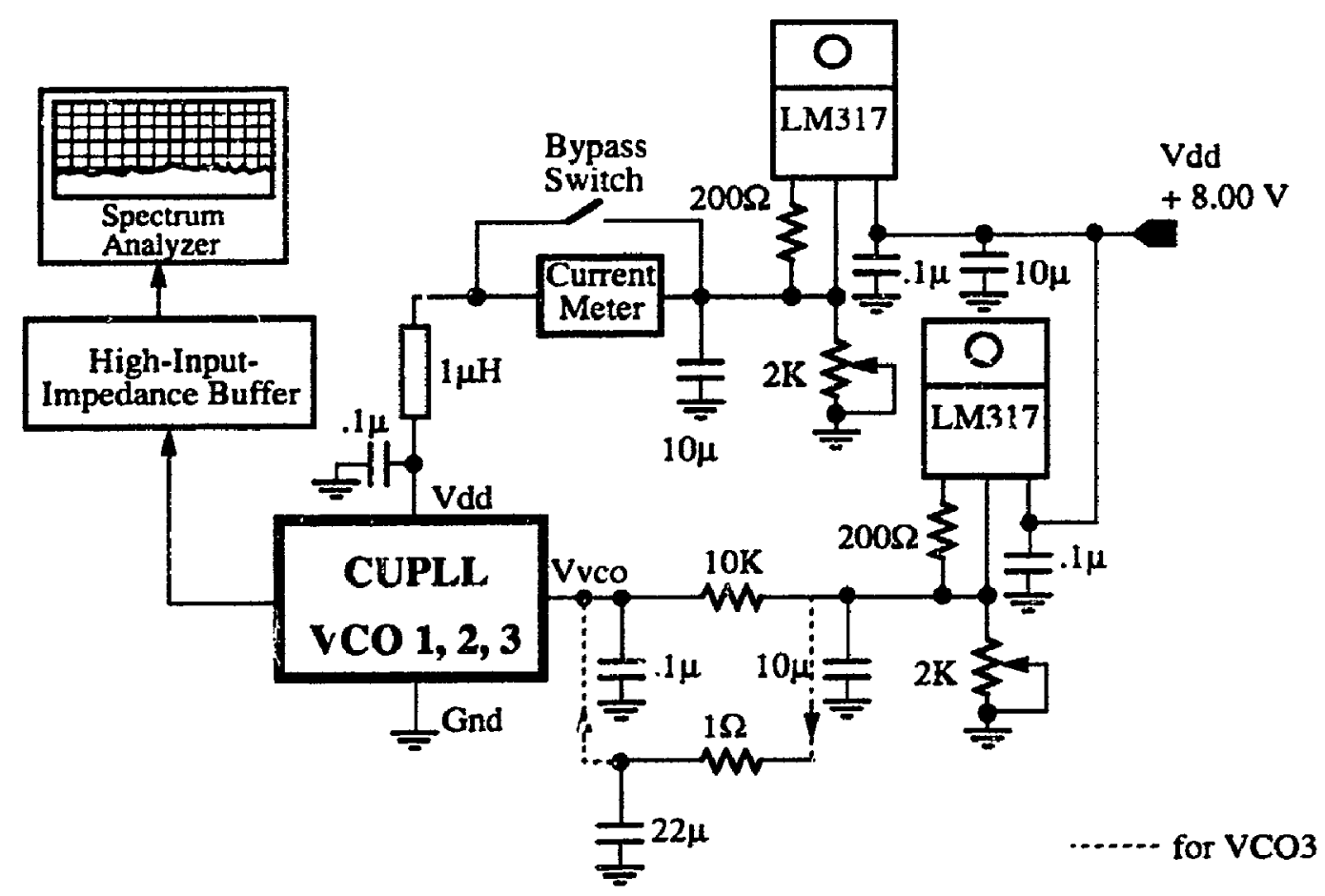

Figure 5.1 CUPLL VCOs' test setup.

The VCO test setup shown in Figure 5.1 provided four major tests as follows:

\subsubsection{VCO transfer characteristic}

This measurement was performed by gradually increasing the tuning voltage or current from the minimum level until the maximum oscillating frequency was found. The tuning voltage for VCO1 and VCO2 was directly adjusted and measured from the de power supply on test board. However, in the measurement of tuning current required for VCO3, a $1-\Omega$ resistor was substituted (shown in Figure 5.1 with a dashed line) and the current level was obtained by measuring the voltage drop across the resistor. The plots of the voltage-frequency transfer characteristics (or VCO tuning range) of the three VCOs are shown in Figure 5.2. For comparison, the simulation results of Chapter 4 are also included. The measured minimummaximum oscillating frequency for VCO1, VCO2 and VCO3 occurred at 320-926 MHz, 225$600 \mathrm{MHz}$ and $100-500 \mathrm{MHz}$ respectively. 


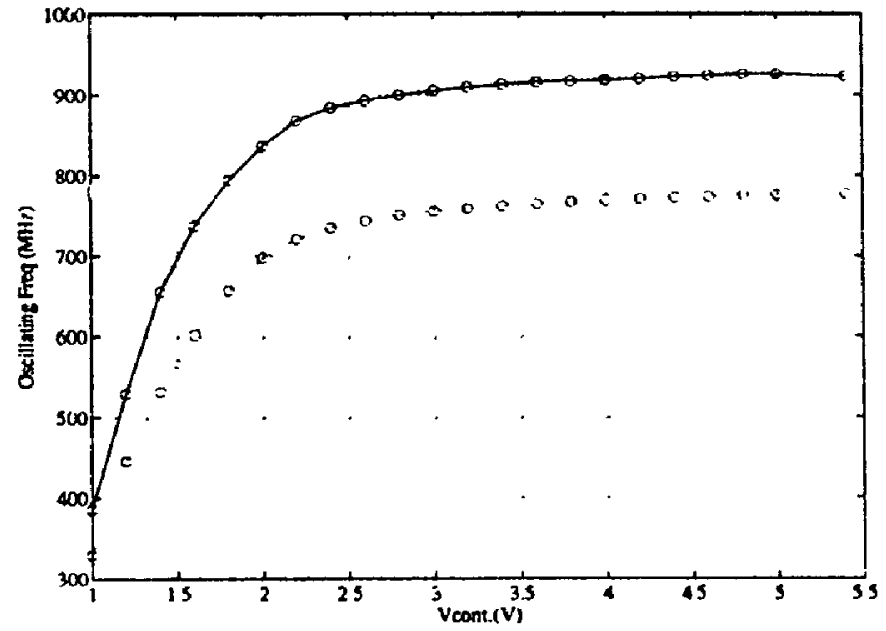

VCO1

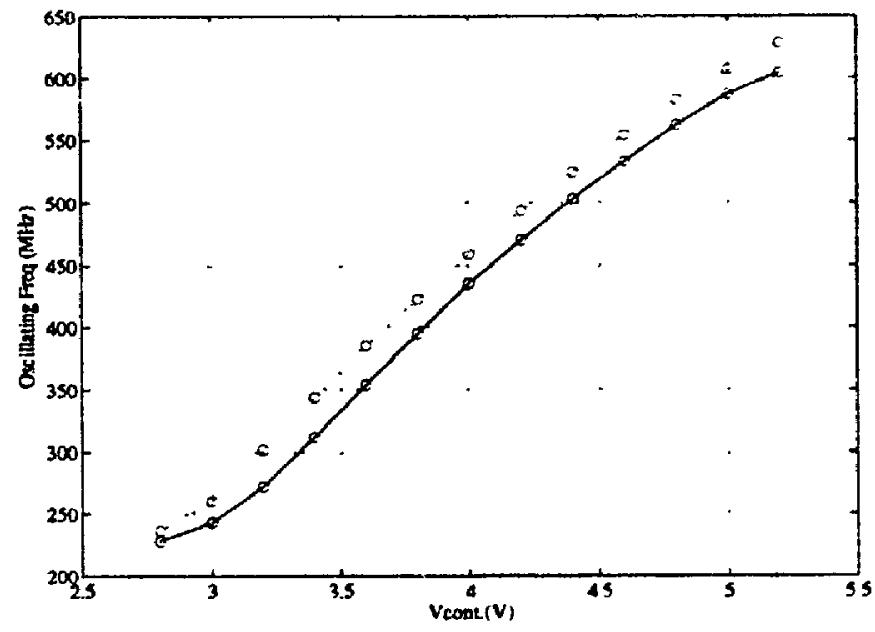

$\mathrm{VCO2}$

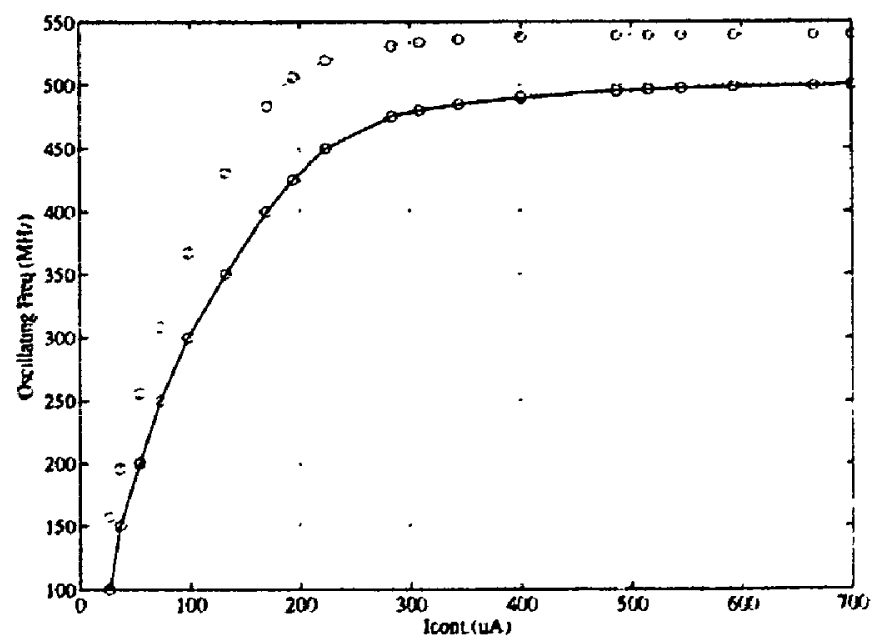

$\mathrm{vCO3}$ 


\subsubsection{VCO spectral output and waveform}

The VCO spectral output, which exhibits the spectral purity of the VCO can be observed using a spectrum analyzer. Figures 5.3 and 5.4 show spectra traces of the VCO output with a frequency span of $1 \mathrm{MHz}$ and a resolution bandwidth of $1 \mathrm{kHz}$. By way of spectral bandwidth comparison, $\mathrm{VCO} 3$ provides the highest spectral purity of the three VCOs.
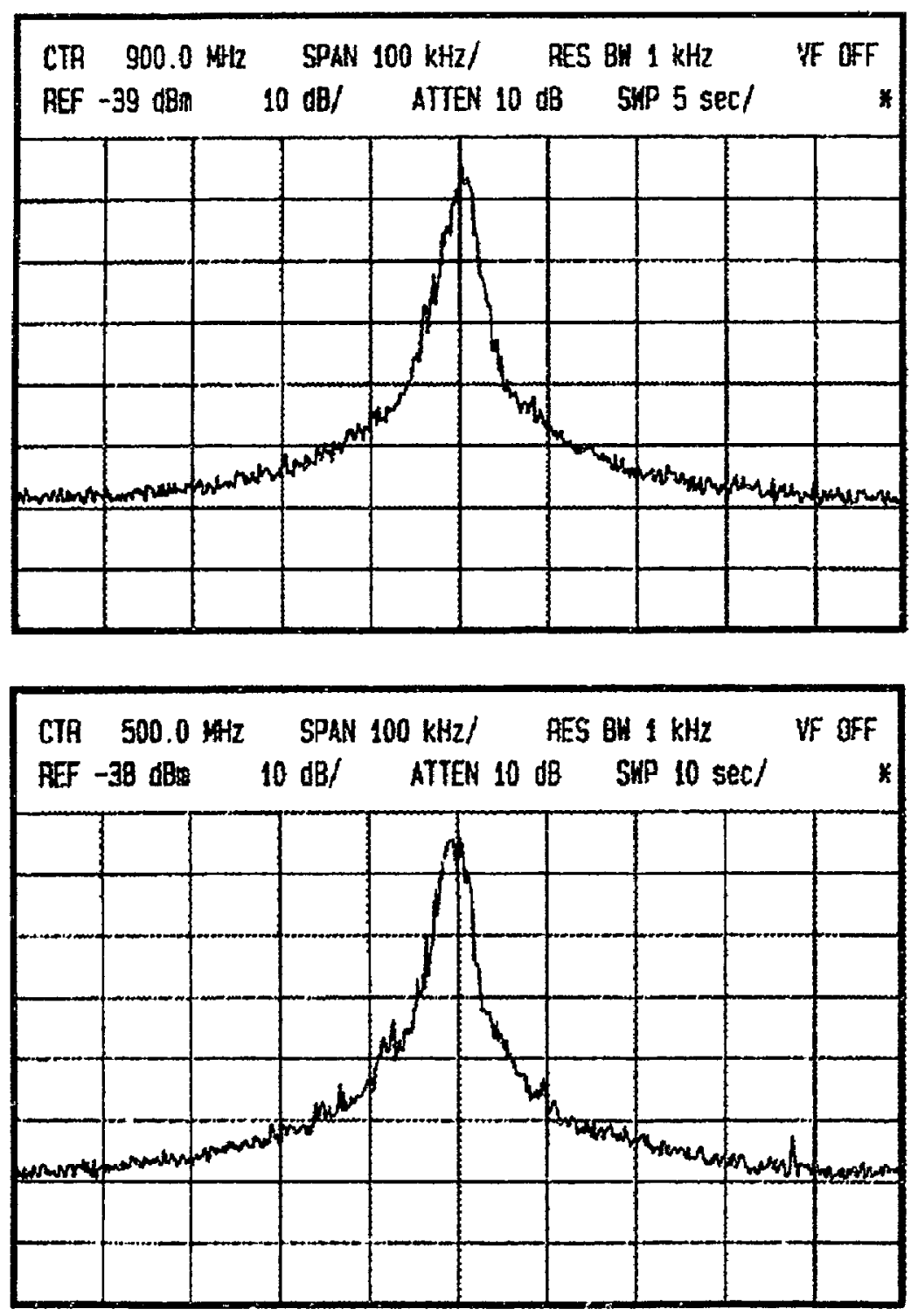

Figure 5.3 Spectra traces of $\mathrm{VCO} 1$ and $\mathrm{VCO} 2$ outputs at $1 \mathrm{kHz}$ resolution bandwidth. 


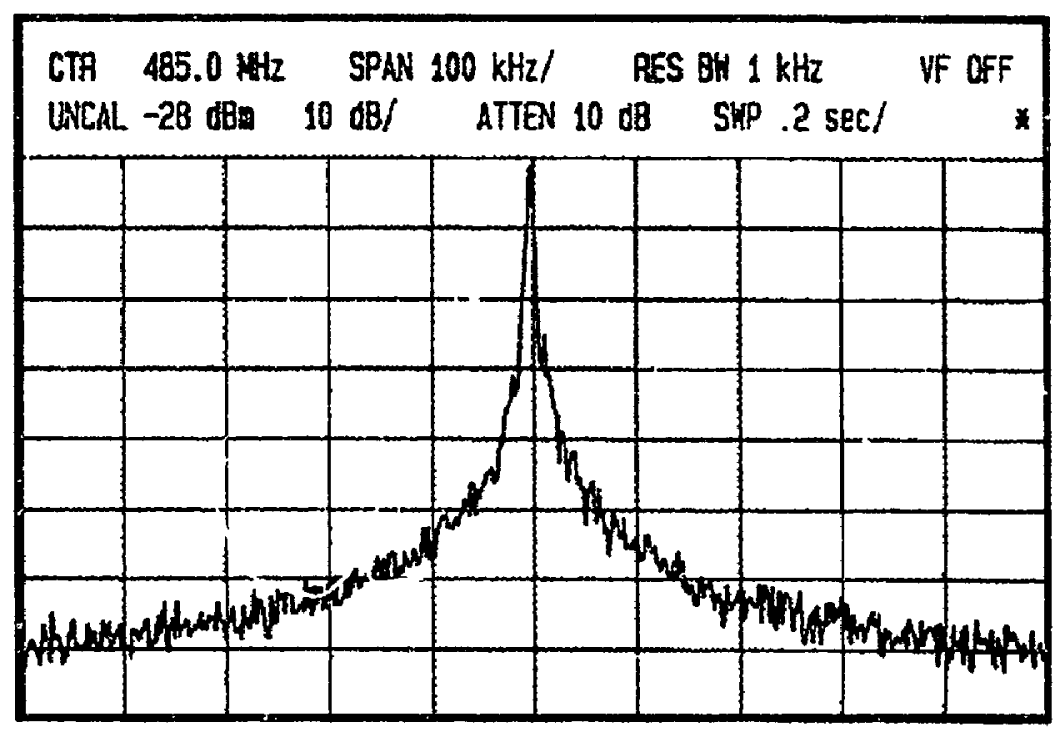

Figure 5.4 Spectra trace of VCO3 output at $1 \mathrm{kHz}$ resolution bandwidth.

Multivibrator circuits, in general, produce iquare wave outputs which consist of a large number of harmonic frequencies considered not suitable for applications involving carrier signal generation in communication systems. However, in the parasitics-capacitorbased ring oscillator design working near a gigahertz frequency, it is apparent that most harmonic frequencies have been shaped by the circuit parasitics. Therefore, the high frequency outputs obtained have a shape similar to the sinusoidal signal shown in Figure 5.5.

\subsubsection{VCO power dissipation}

CMOS circuits dissipate dynamic power depending very much on their load capacitance. Since all VCO output signals have to be brought off chip through analog output pads, these VCOs certainly cannot avoid the excess power dissipated from the effect of large pad parasitic capacitance. 


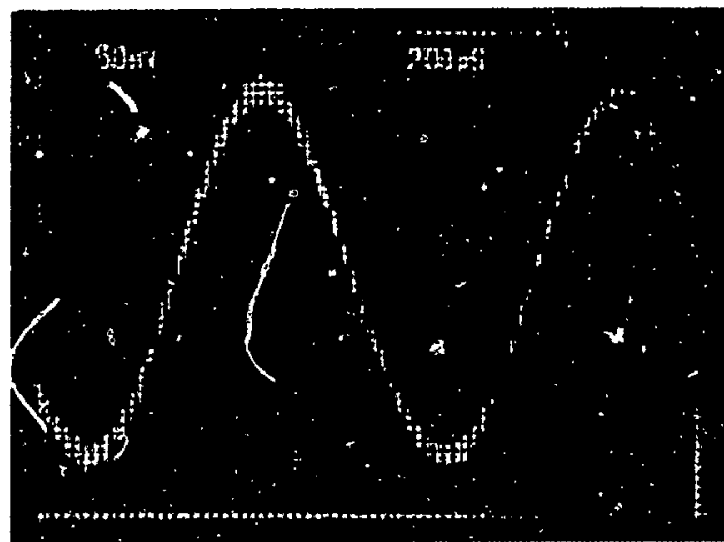

VCO1

$900 \mathrm{MHz}$

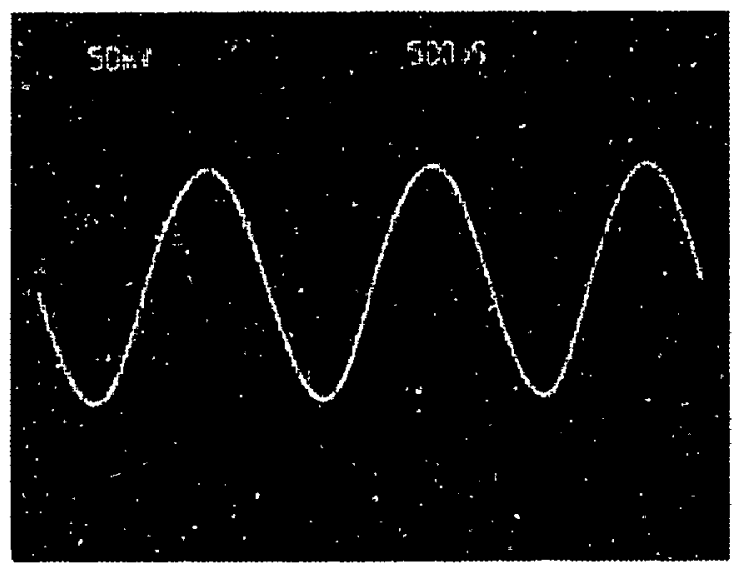

vCO1

$600 \mathrm{MEz}$

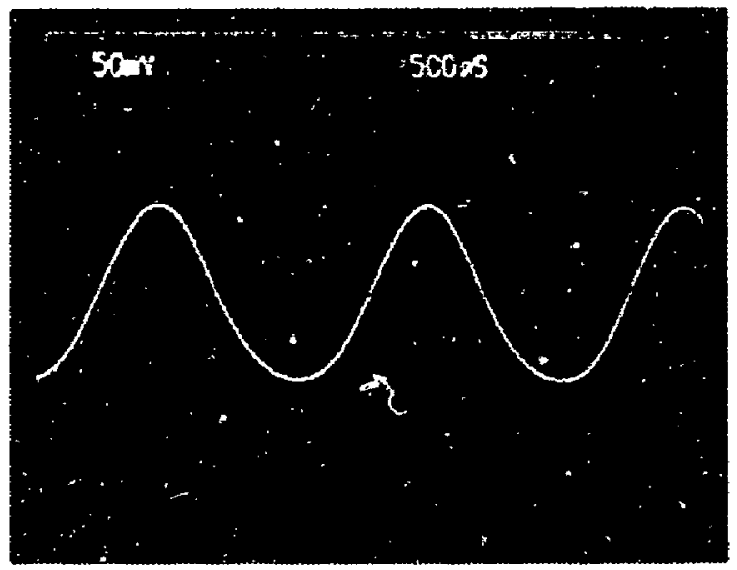

VCo1

$500 \mathrm{MHz}$

Figure 5.5 Oscilloscope traces for VCO waveforms. 
From the layout extraction, the approximate capacitance created by the pad was 1.77 pF. In addition, package capacitance also adds to the loading effect on the circuit. The IC packaging ?7ta of PGA-68 [77] indicates that more than 1 pF of capacitance is expected. These values of capacitance can significantly increase the total power dissipation for circuits working at very high frequencies. If circuits are not required to drive large loads, then buî́er circuits may be eliminated and power dissipation could then be reduced.

This section reports on the performance of the fabricated VCOs for different oscillating frequencies. The same measurement setup shown in Figure 5.1 was used for this measurement. Power consumption is simultaneously measured indirectly by a digital micro-ampere meter.

Figure 5.6 shows the power consumed by each VCO at different oscillating frequencies. The top and middle curves, the power consumed by $\mathrm{VCO} 3$ and VCO2, show that the dissipated power is a linear function of frequency. $\mathrm{VCO} 2$ and $\mathrm{VCO} 3$ dissipate a maximum jower of $14 \mathrm{~mW}$ and $20.2 \mathrm{~mW}$, respectively.

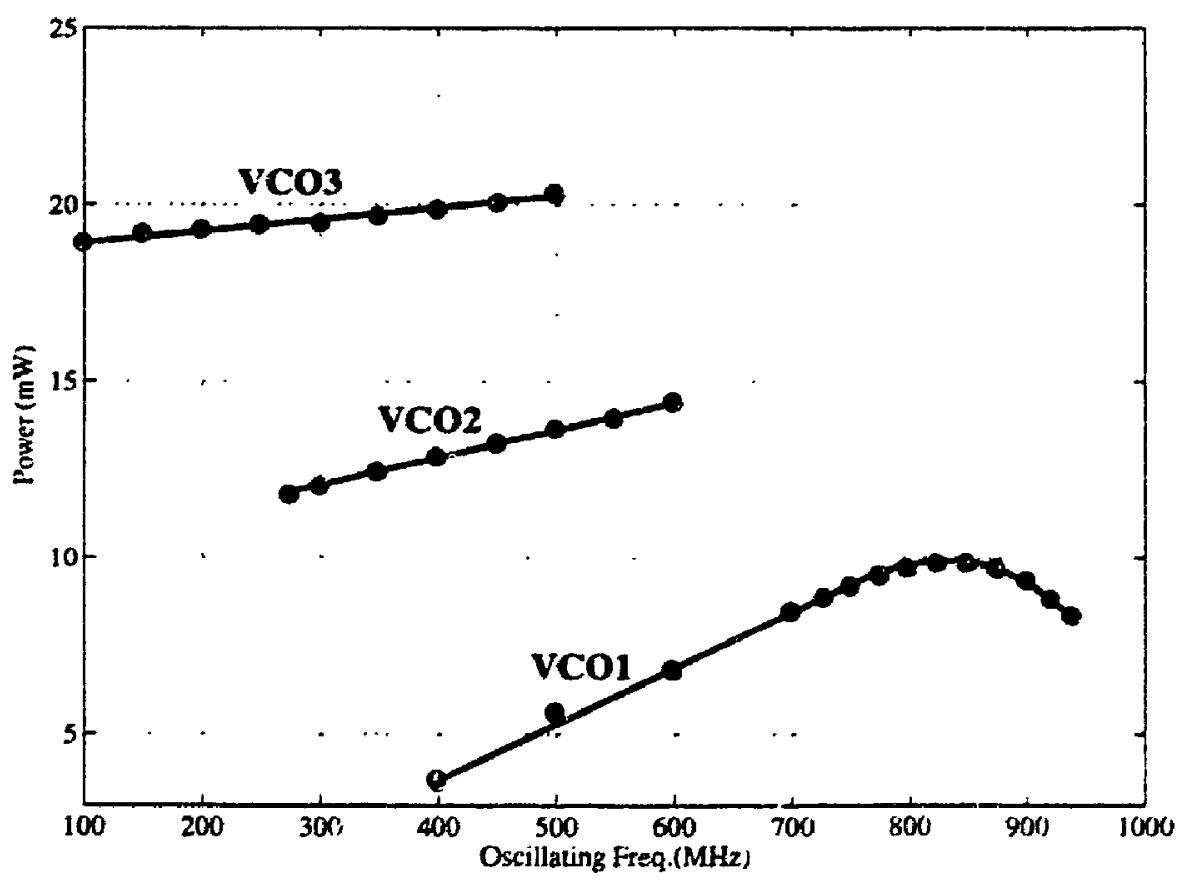

Figure 5.6 Power dissipation as a function of oscillating frequency. 
The bottom curve, VCOl power dissipation, exhibits a nonlinear function. This can be explained by the state of circuit operation. According to simuiation results reported in Chapter 4, VCOI at oscillation frequencies below $620 \mathrm{MHz}$, generates an output at a full swing of 5 volts. As the oscillating frequency increases the output amplitude decreases in size.

Test results of VCOI show that the fabricated chip oscillates 16 percent faster than the results obtained from simulations. Thus, the frequency at which power dissipation begins to decline occurs for oscillating frequencies beyond $820 \mathrm{MHz}$. The VCO maximum power dissipation is measured at $9.4 \mathrm{~mW}$. However, if the power dissipated by pad capacitance and buffer $(3.92 \mathrm{~mW}$, at $820 \mathrm{MHz})$ are excluded, then the predicted core circuit would consume only $5 \mathrm{~mW}$ of total power.

\subsubsection{Sensitivity to power supply variations}

To evaluate the VCO sensitivity to posver supply variation, all VCOs were measured in terms of their oscillation frequencies as a tunction of supply voltage (Vdd). The results are shown in Figure 5.7.

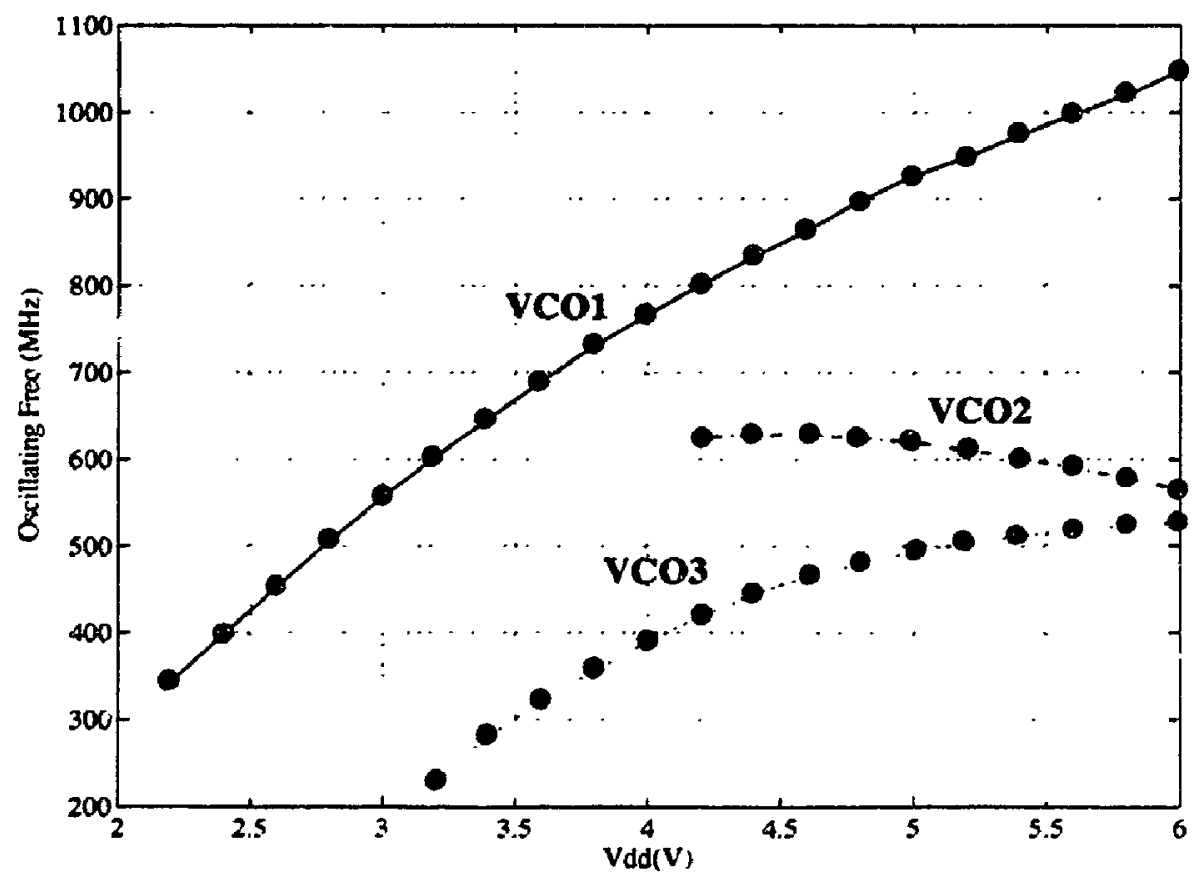

Figure 5.7 Frequency of oscillation as a function of supply voltage. 
VCO1, the simplest ring structure, shows the highest sensitivity to the power supply variation. VCO2 demonstrates good stability regardless of the variations in supply voltage. And $\mathrm{VCO} 3$ is less sensitive when the power supply approaches 4.5 volts and beyond. All these results show good agreements with the simuiated results presented in Figure 4.15 a.

\subsubsection{VCO phase noise measurement}

A phase noise measurement system was used to characterize the phase iistability of the oscillators. This method is recognized as a standard technique in determining VCO quality in terms of short term frequency stability (known as phase noise). The measured results provide a single-sided phase noise level that is relative to the carrier level measured at the specified offset from the carrier in a 1- $\mathrm{Hz}$ bandwidth, and expressed in units of $\mathrm{dBc} /$ Hz. All VCO phase-noise outputs were measured using this special phase noise measurement system (HP3048A) [78].

The test equipment required for this measurement is shown in Figure 5.8. The VCO output was coupled via a $22-\mathrm{nF}$ capacitor before being connected to a terminal placed as close as possible to the socket pir. A $17 \mathrm{dBm}-50-\Omega$-input amplifier was required in this test due to the low power le vel of the VCO output, which could not directly drive the phase noise interface equipment.

In order to characterize the performance at worst case, the frequency of oscillation was chosen to be close to the maximum possible frequency for all VCOs, that is VCO1 at $900 \mathrm{MHz}, \mathrm{VCO} 2$ at $550 \mathrm{MHz}$ and $\mathrm{VCO} 3$ at $475 \mathrm{MHz}$.

In this test, the VCO becomes a component in a PLL synthesizer configuration and the VCO tuning input is driven by a dc source generated from the phase-noise measurement system. Under lock condition, the system evaluates the phase noise of the VCO by measuring the residual FM (-3dB points) at different bandwidths and stores the data in the control unit. The measured results are then displayed in a single-sided plot 
where the $X$ axis represents the level of the phase noise in $\mathrm{dBc} / \mathrm{Hz}$ and the $\mathrm{Y}$ axis represents the frequency offset from the center frequency of the measured VCO. All VCOs were similarly tested and the phase noise plots are shown in Figure 5.9.

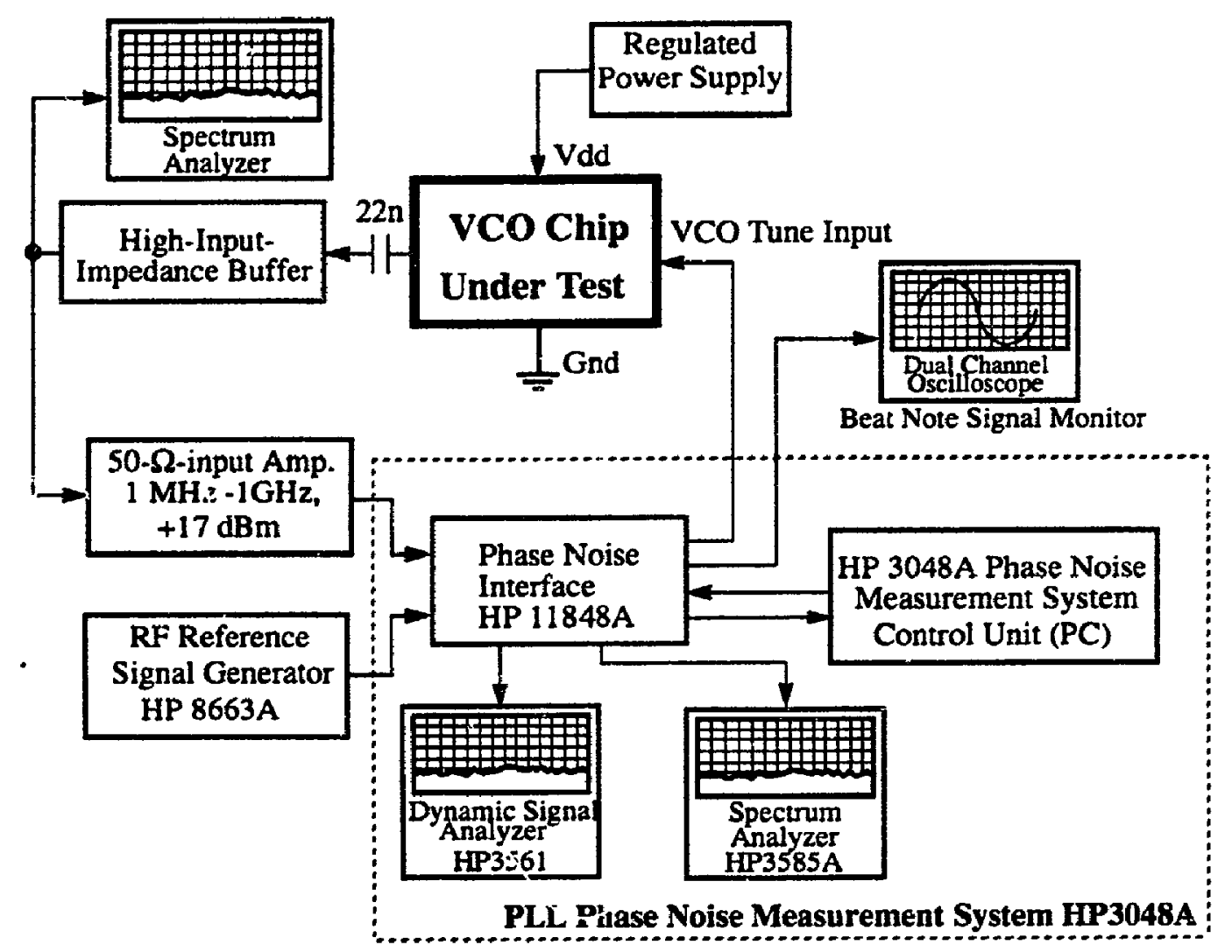

Figure 5.8 Test bed \& setup for VCO phase r.rise characterization.

VCO1, VCO2 and VCO3 phase noise was measured and plotted on the top, middle and bottom charts respectively. The phase noise at $100 \mathrm{kHz}$ offset from carrier frequencies shows that VCO1 had the highest noise levels at $-83 \mathrm{dBc} / \mathrm{Hz}$, followed by $\mathrm{VCO} 2$ at -87 $\mathrm{dBc} / \mathrm{Hz}$. VCO3 delivered the lowest phase noise response among the three at $-90 \mathrm{dBc} / \mathrm{Hz}$.

Note that the phase noise measurements obtained from the test setup were predicted to be worse than the actual values. This can be blamed on the poor circuit shielding. Moreover, the additional amplifier and interconnections also increased noise to the VCO outputs. 

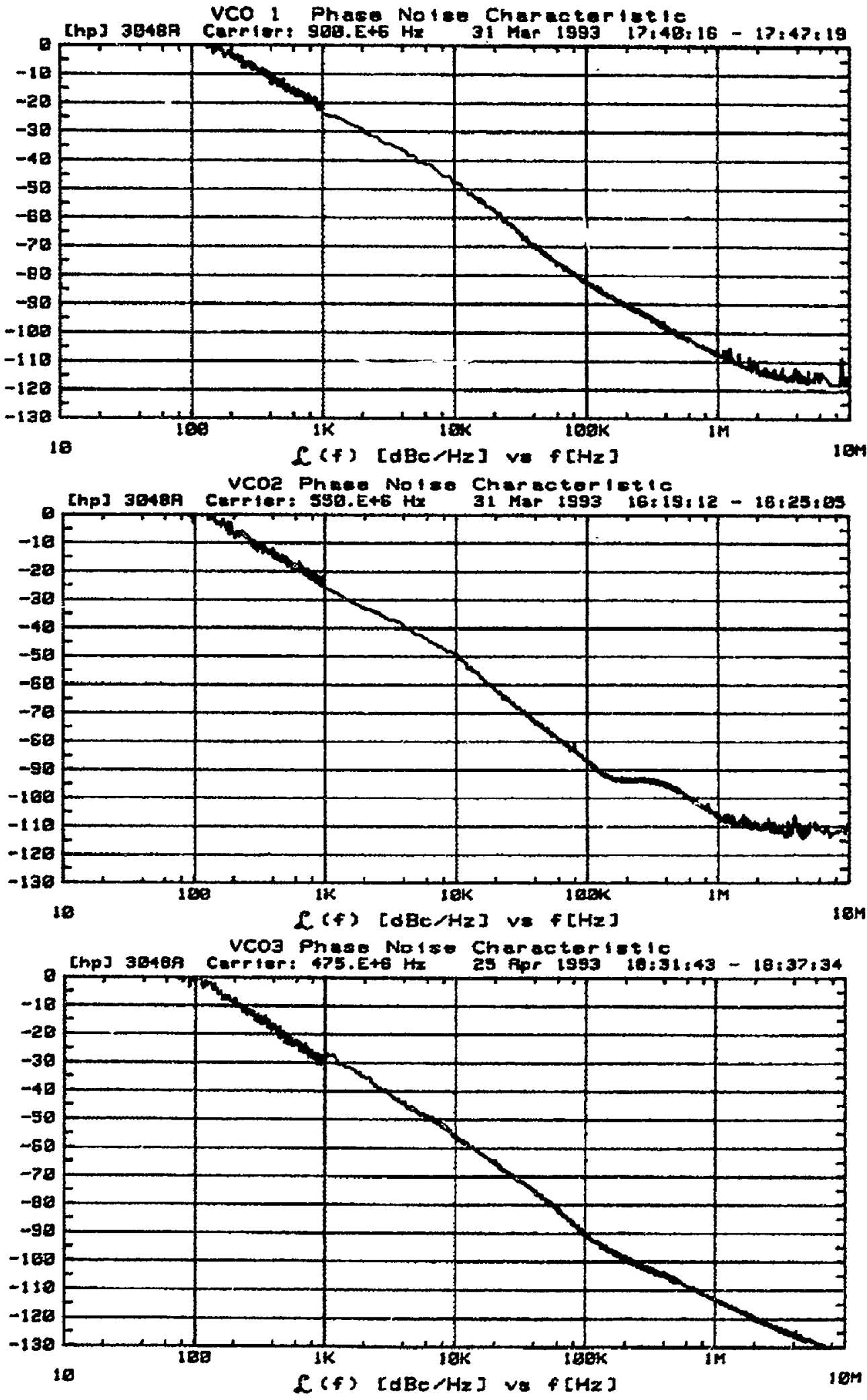

Figure 5.9 Measured VCO1, VCO2, VCO3 residue FM/SSB phase noise using HP3804A. 


\subsubsection{Test setup for verification of phase noise prediction technique}

Verification of the phase noise prediction technique was performed using the configuration depicted in Figure 5.10. This test was carried out in the same manner as proposed in Section 4.4.3. The test bed consisted of the VCO circuit board, a bias tee circuit and other necessary measuring equipment.

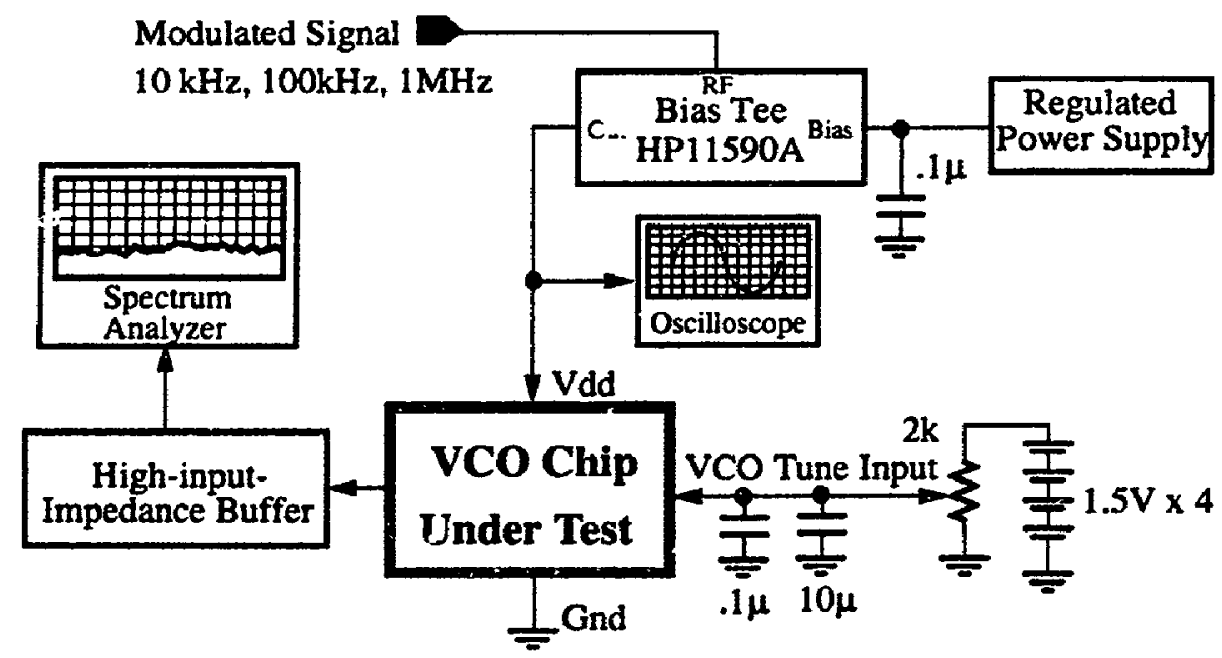

Figure 5.10 Test setup for verification of phase noise prediction technique.

The VCO's source supply (Vdd) was ac modulated through the bias tee circuit, while the VCO tuning input is driven by a clean dc source constructed from adjustable battery cells. The test setup assured that no other noise source is introduced to the VCO under the test except for the noise on the power rail.

A single tone sinewave signal of $13 \mu \mathrm{V}$ at $10 \mathrm{kHz}, 100 \mathrm{kHz}$ and $1 \mathrm{MHz}$ was added to the 5-volt de regulated supply for testing. The VCO output spectra with $100 \mathrm{kHz}$ added signal obtained by the spectrum analyzer are shown in Figures 5.11 through 5.13 . 
The measured results demonstrate that VCO1 is the most susceptible to power supply noise. This is indicated by the presence of the two wide side bands at $100 \mathrm{kHz}$ offset from the center frequency. The corresponding results for $\mathrm{VCO} 2$ and $\mathrm{VCO} 3$ indicate that VCO3 shows the highest degree of noise rejection as shown by the sharp modulated side bands in Figure 5.13. This confirms that the proposed ac analysis for a CMOS oscillator presented in Section 4.1 is valid due to the high degree of correlation between the simulated and measured results for the three VCO structures (see Section 4.4.2).

These measurements also support the approach using VCO phase noise simulations, proposed in Section 4.4.3. The concept has demonstrated credibility through the design of a CMOS oscillator.

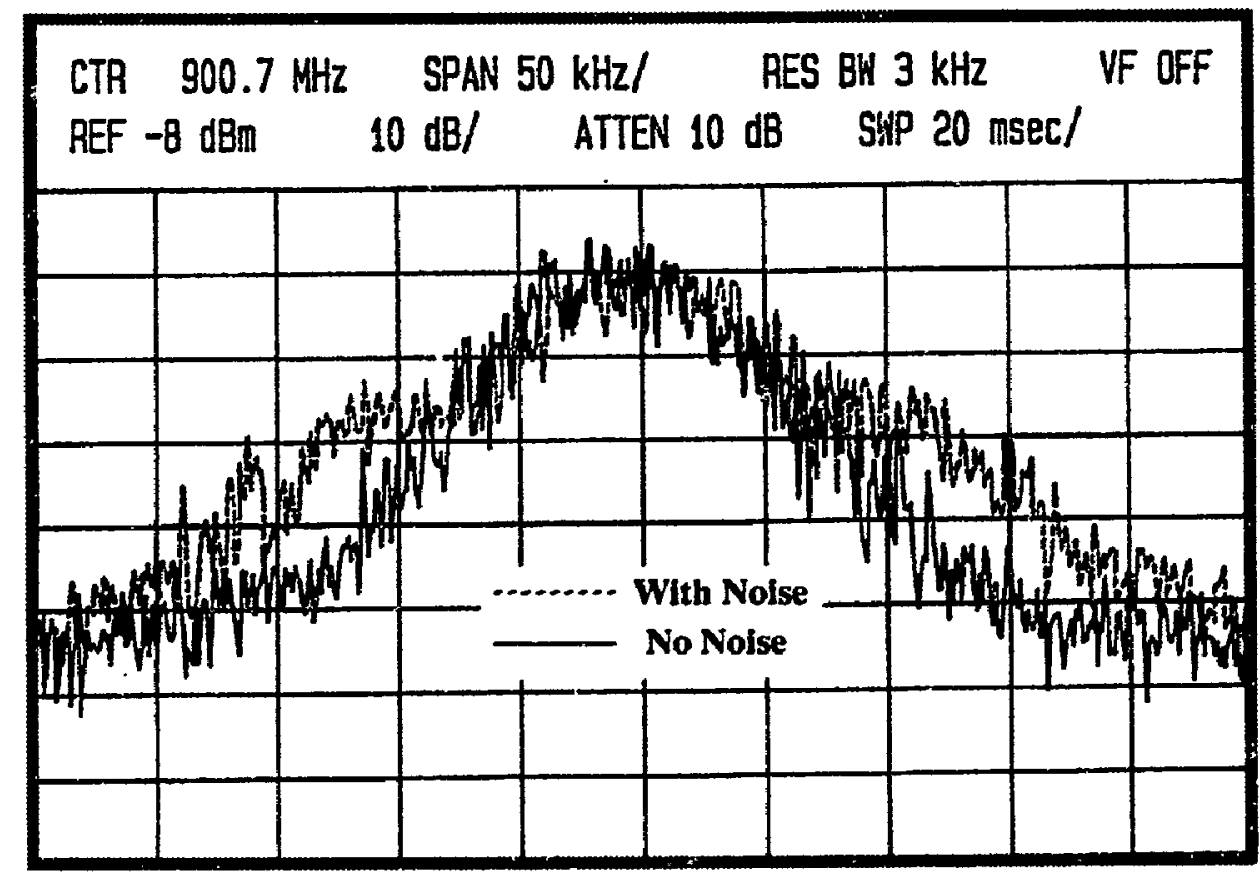

Figure 5.11 VCO1 spectra trace at $900 \mathrm{MHz}$ before and after adfed noise. 


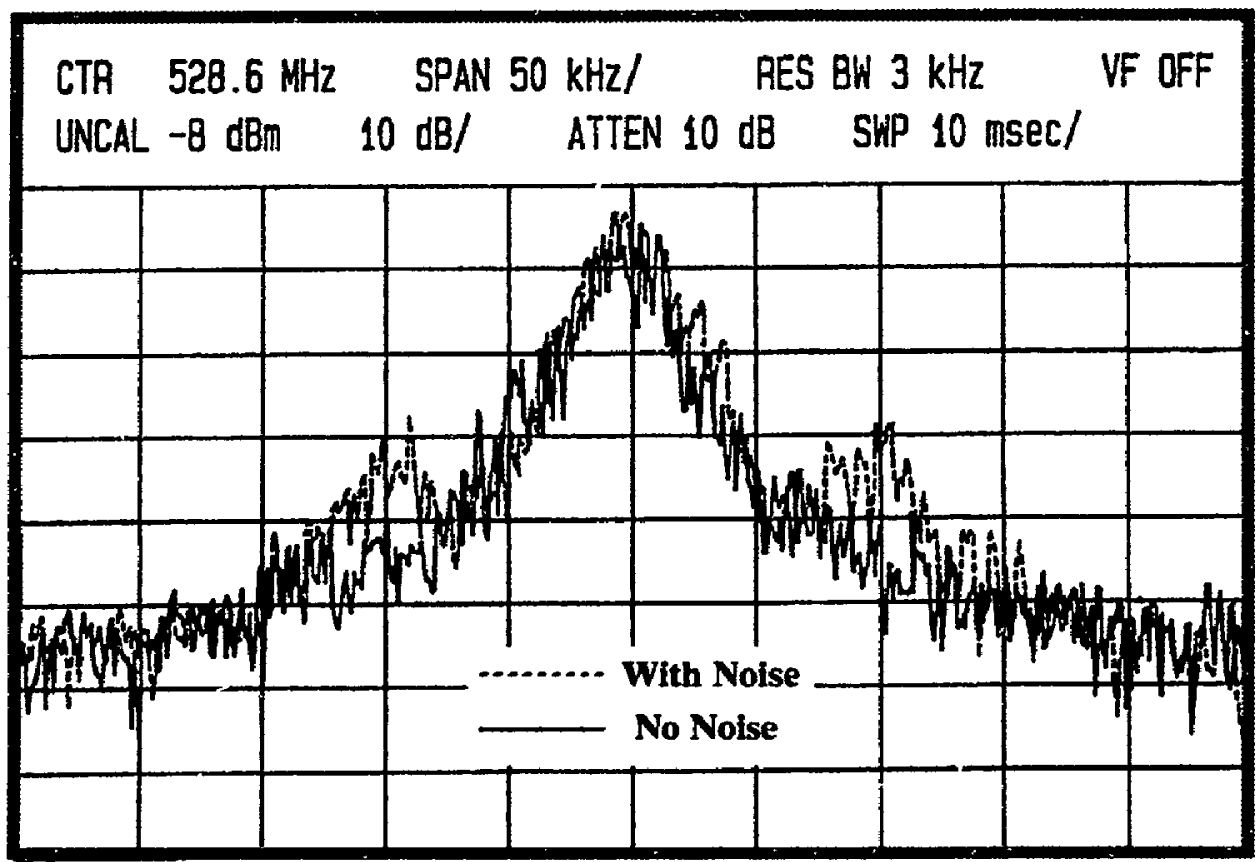

Figure 5.12 VCO2 Spectra trace before and after added noise.

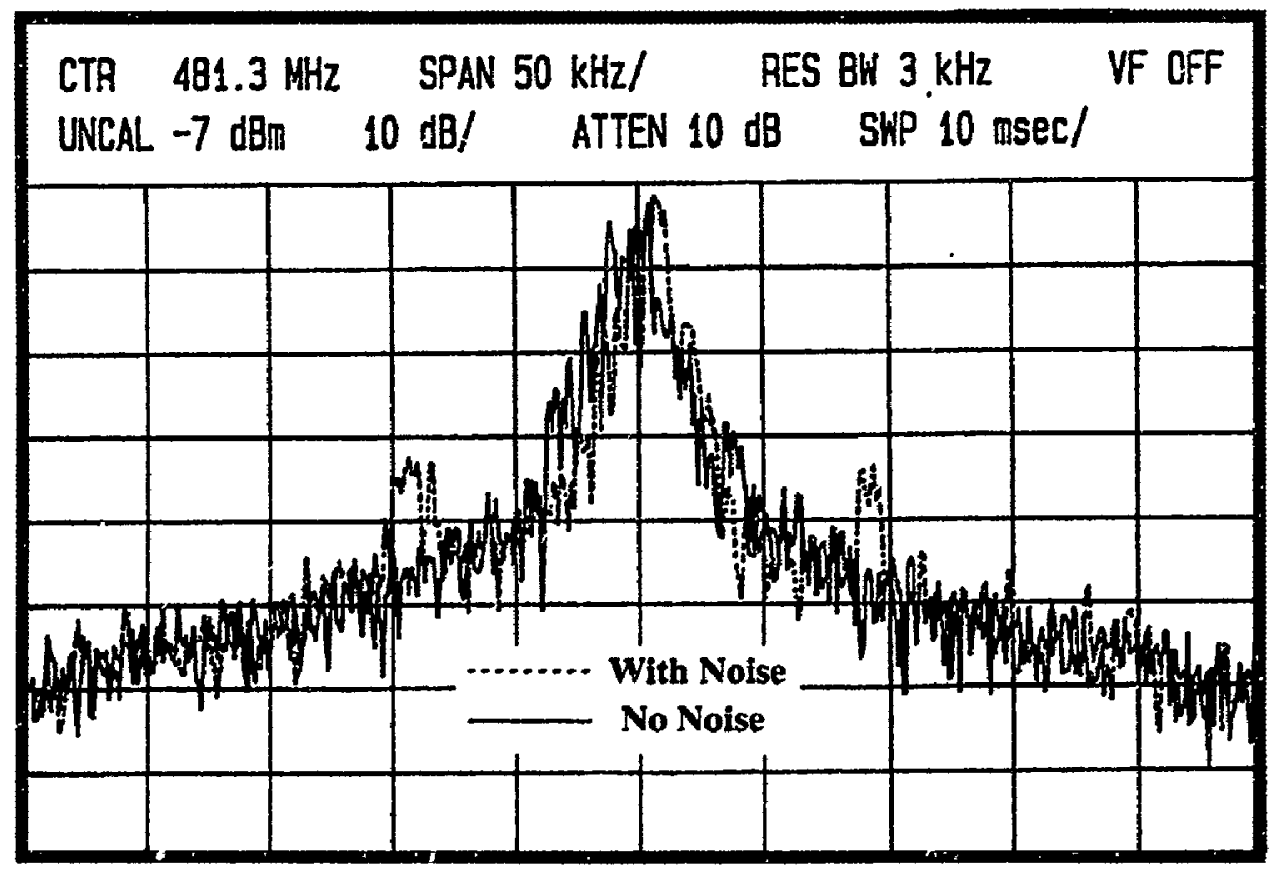

Figure 5.13 Modulated spectra output of VCO3, demonstrating best noise rejecting capability. 


\subsubsection{Phase-frequency detector (FFD) test setup}

This section presents the measured results for the monolithic CMOS phasefrequency detector in the CUPLL chip. Measurements were made to characterize the functionality and the performance of the phase-frequency detector.

Figure 5.14 shows the test bed and equipment required for measurement of the linear phase detector range. The PFD is driven by two input sources, (i) a reference signal and (ii) a controllable delayed signal. Two stable high-frequency signal generators are used as detector inputs, while an audio signal generator is used to modulate one input. The delayed signal is synchronized to the reference signal generator. The audio generator then modulates the delay input port over a range of phase difference. To ensure square waveforms, two inverter buffers are used as interface circuits.

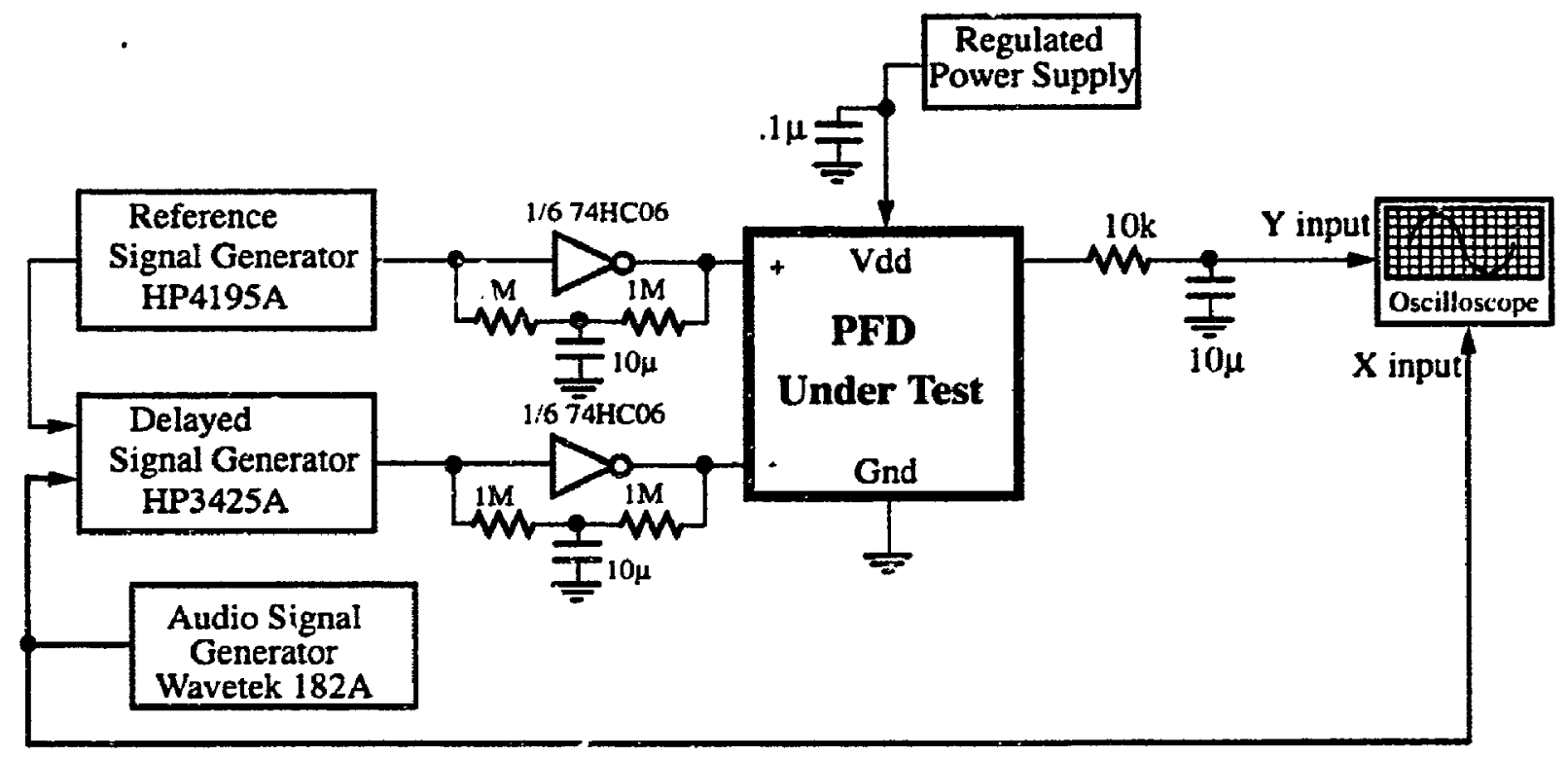

Figure 5.14 Test sefup for measurement of phase-frequency detector cinaracteristic.

The signal obtained on the PFD output is a pulse train whose duty cycle corresponds to the phase difference between the two inputs. This pulse is filtered by a passive RC network. 
The filtered output is connected to the $\mathrm{Y}$ input of the oscilloscope, while the $\mathrm{X}$ input is driven by the modulated signal representing the delayed value of the two inputs. The PFD phase detection range was measured and recorded at three different frequencies.

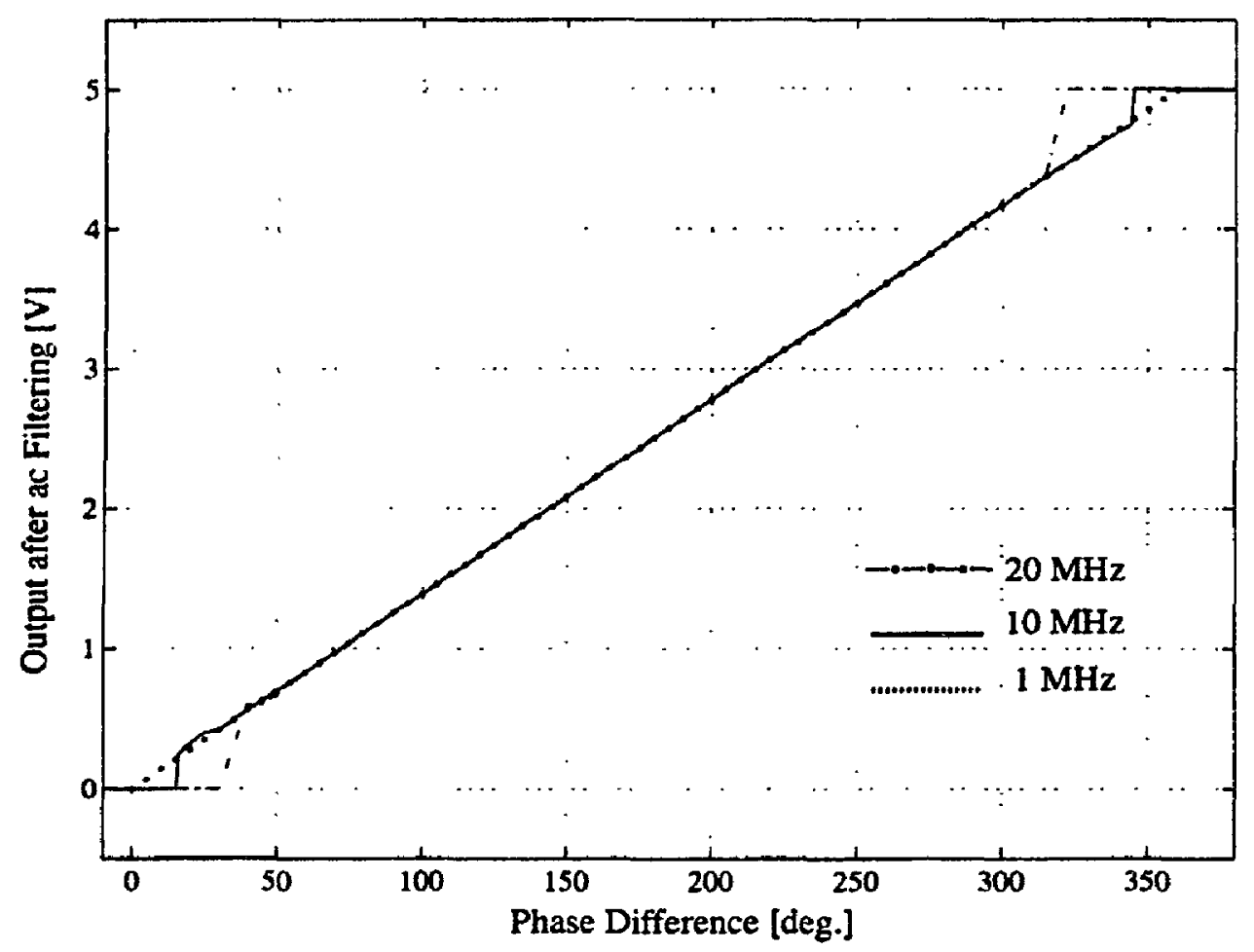

Figure 5.15 Measured phase-frequency characteristic.

Figure 5.15 shows the three plots demonstrating the phase-frequency detector characteristics. As expected, the PFD shows a linear phase detection range with dead zones at both ends. The dead zone width increases in proportion to the increase of input frequencies. 'This measurement did not include frequencies above $20 \mathrm{MHz}$ due to the limit on the maximum frequency of the reference signal generator (HP 4195A) used in the test setup. However, it is expected that the PFD would still function correctly at higher frequencies and the width of the dead zone will also increase. 


\subsubsection{Performance summary of CUPLL}

\begin{tabular}{|c|c|c|c|c|}
\hline & \multicolumn{3}{|c|}{ This Work } & $\begin{array}{c}\text { The Fastest } \\
\text { Reported } \\
\text { CMOS VCO }\end{array}$ \\
\hline $\begin{array}{c}\text { Reference } \\
\text { (Gate Length) }\end{array}$ & $\begin{array}{c}\text { VCO1 } \\
(1.2 \mu \mathrm{m})\end{array}$ & $\begin{array}{c}\text { VCO2 } \\
(1.2 \mu \mathrm{m})\end{array}$ & $\begin{array}{c}\text { VCO3 } \\
(1.2 \mu \mathrm{m})\end{array}$ & $\begin{array}{c}\text { Abidi[90] } \\
(1.0 \mu \mathrm{m})\end{array}$ \\
\hline $\begin{array}{c}\text { Max. Operating Frequency } \\
\text { (MHz) }\end{array}$ & 926 & 600 & 500 & 320 \\
\hline Tuning Range (MHz) & 606 & 375 & 400 & 50 \\
\hline $\begin{array}{c}\text { Phase Noise at 100KHz offset } \\
\text { (dBc/Hz) }\end{array}$ & -83 & -87 & -90 & - \\
\hline Power (mW) at Fmax & $7.4 *$ & $14.0 *$ & $20.2 *$ & - \\
\hline Nominal Vdd (V) & 5 & 5 & 5 & - \\
\hline
\end{tabular}

Table 5.1 Summarized performance from the VCO design.

* Including the output buffer circuit, PAD and package capacitance $(\approx 2.77 \mathrm{pF})$

\subsection{Single-chip CMOS fractional-N synthesizer (CUPL2)}

This section reports the performance evaluation of a PLL based frequency synthesizer constructed from all CMOS PLL components from the CUPL2 fabricated chip, excluding the loop tilter. The chip was a second iteration design using the test results presented in Section 5.1. The design included a frequency divider on the same chip. All components except the loop filter were laid out with some modifications made to the frequency divider and phase-frequency detector to accommodate the VCO performance as well as to achieve the best overall noise performance.

\subsubsection{Design review}

The selected components, design considerations and a brief description of the circuits for the CUPL2 implementation are described below: 
- VCO: VCO1 was chosen for this implementation for two reasons. (i) the selected frequency range more closely matches the digital cellular transmission standards (GSM, CT2+ and IS-54), and therefore, results can be directly compared with standard designs implemented in different technologies. (ii) it exhibits the highest maximum oscillation frequency among the three VCOs.

From the test results obtained from CUPLL, the maximum frequency of operation for VCO1 was $926 \mathrm{MHz}$. Its intended nominal operation was designed for 800 to $900 \mathrm{MHz}$.

- Frequency divider: A divide-by-15/16 fractional divider proposed in [5] was chosen for this implementation because the design was well suited for a high-speed and low-power requirement. As well, the divider was first designed using the same process as the VCO, resulting in less complexity for layout adjustment and development time.

Key specifications for the divider are summarized here briefly (for additional details see [5]). The input frequency range to the divider is from $700 \mathrm{MHz}$ to $1.4 \mathrm{GHz}$ for proper operation, and the input amplitude is required to be less than $600 \mathrm{mV}$. The minimum input signal level is a function of the bias level supplied to the divider, and therefore minimum input signal level must be determined during testing.

- Phase detector: The design of a single ended no-dead-zone phase-frequency detector (PFD) was modified to a fully balanced version for this implementation as common mode noise problems were of prime concern. Two individual PFDs were laid out symmetrically, and cross coupled at the inputs to achieve a balanced structure.

From the test results, the phase detector is capable of operating at $20 \mathrm{MHz}$ with a linear phase detection range of 270 degrees. It is possible that operating the PFD at higher frequencies could make the PLL lose lock easily.

- Loop filter: To demonstrate the functionality of a first implementation, a typical second-order loop filter using an active integrator filter with phase lead compensation was 
employed. However, to support the use of a balanced structure PFD, a differentially summed output integrator was required. Also, prefiltering was added to assist loop stability.

\subsubsection{Closed loop parameters}

This section reports on the calculation of loop parameters and component values. Conventional closed loop equations can be found in many text books on PLL design [11. $12,16]$.

Information obtained from measured results of VCOl and PFD were used to implement the loop filter for closed loop PLL testing. The second-order loop equations are summarized below.

The closed loop transfer function, $\frac{\theta_{o}(s)}{\theta_{1}(s)}$. can be obtained from the standard secondorder configuration (see Figure 3.1) and is given by

$$
\frac{\theta_{o}(s)}{\theta_{i}(s)}=\frac{\frac{K_{\phi} K_{v c o}}{\tau_{1}}\left(1+s \tau_{2}\right)}{s^{2}+s \frac{K_{\phi} K_{v c o} \tau_{2}}{N \tau_{1}}+\frac{K_{\phi} K_{v c o}}{N \tau_{1}}}
$$

where $\tau_{1}$ and $\tau_{2}$ are time constants of the lag-lead integrator filter shown in Figure 5.16.

From Eq. 5.1, the loop natural frequency $\omega_{n}$ is given by

$$
\omega_{n}=\sqrt{\frac{K_{\phi} K_{v c o}}{N \tau_{1}}}
$$

and related to $B_{L}$, the $-3 \mathrm{~dB}$ point of the closed loop bandwidth by

$$
\omega_{n}=\frac{B_{L} \pi}{\zeta}
$$


The damping ratio $\zeta$ is represented by

$$
\varsigma=\frac{\omega_{n} \tau_{1}}{2}
$$

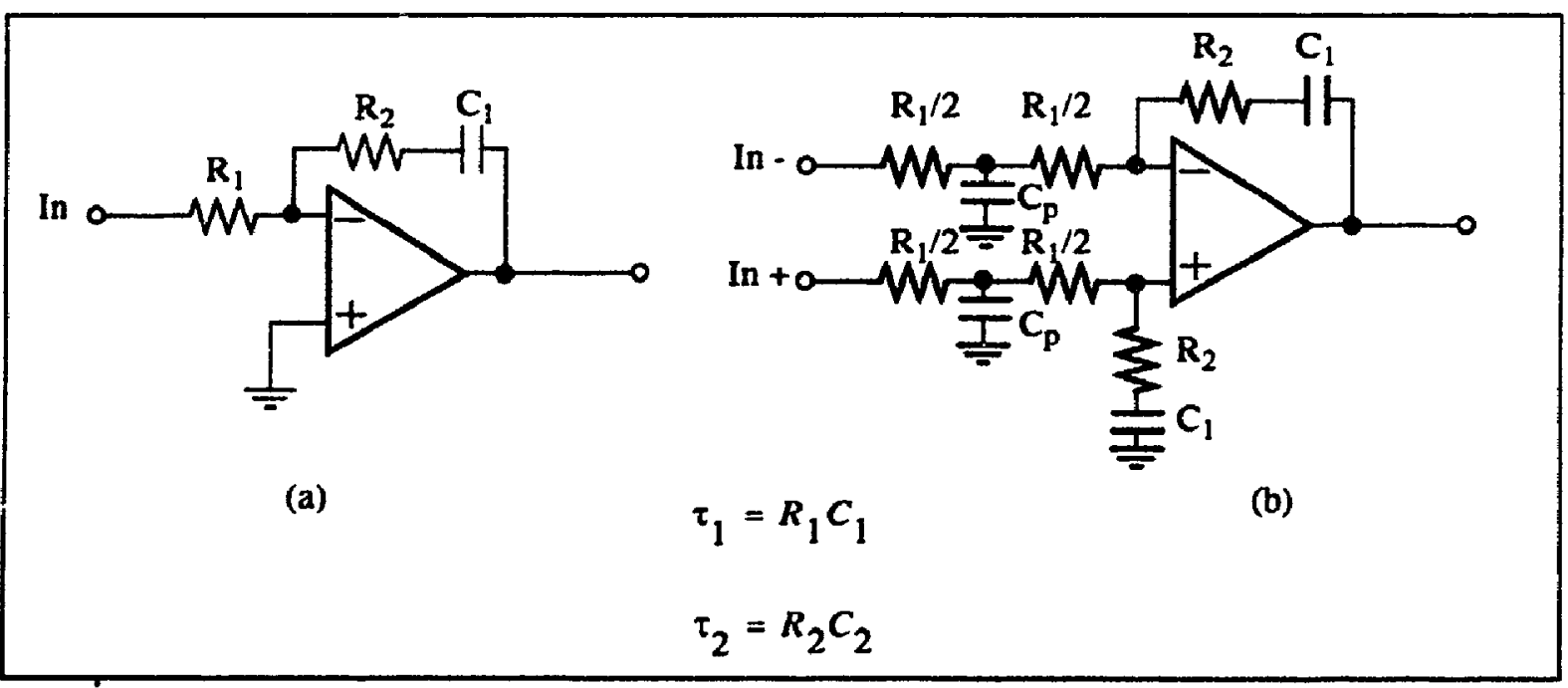

Figure 5.16 (a) Loop filter, single ended, (b) differential configuration with prefiltering.

If the forward gain of the loop is set to constant, $K$, where $K=\frac{K_{\phi} K}{N}$ then $\tau_{1}$ and $\tau_{2}$ can be expressed by

$$
\begin{gathered}
\tau_{1}=\frac{K}{\omega_{! \ell}^{2}} \\
\tau_{2}=\frac{2 \zeta \omega_{n} \tau_{1}}{K}
\end{gathered}
$$

As suggested a prefiltering network should be added in order to enhance the settling time and protect the opamp from slew limiting effect (nonlinear integration). The cutoff frequency of the additional pole will be selected at the testing stage. However, the value 
can be initially set at twenty times the frequency of the loop bandwidth $\left(B_{L}\right)$ of the PLL and the final value will be selected when the loop is closed.

The design goal for this experiment was that the overall phase noise of the PLL must meet EIA cellular standards as described in Chapter 3. A $-65 \mathrm{dBc} / \mathrm{Hz}$ phase noise at 45 $\mathrm{kHz}$ offset from the center carrier must be satisfied. Consequently, the loop filter must take into account the VCO phase noise performance.

The design parameters are as follows:

- $K_{\mathrm{q}}=\frac{5}{2 \pi}=0.796 \mathrm{~V} / \mathrm{rad}$ and $K_{v c o}=35.7 \mathrm{MHz} / \mathrm{V}\left(\right.$ or $\left.2.243 \times 10^{8} \mathrm{rad} / \mathrm{sec} \cdot \mathrm{V}\right)$

- $B_{L}=100 \mathrm{MHz}$, and $\zeta=2$ (overdamped for ease of lock)

- $N=64$, (with additional divide-by-4 divider cascaded with the on-chip divideby- 6 divider)

Selecting $R_{1}=20 \mathrm{k} \Omega$, gives $C_{1}=5.652 \times 10^{-9} \mathrm{~F}$, and by solving for $\tau_{2}$ in Eq. (5.6) yields $R_{2}=4.506 \times 10^{3} \Omega$ The actual values closest to standard component values for $R_{2}$ and $C_{1}$ are $4.7 \mathrm{k} \Omega$ and $6.8 \mathrm{nF}$ respectively. For prefiltering, $C_{\mathrm{p}}$ was chosen as $3.9 \mathrm{pF}$ according to the selected pole frequency.

\subsubsection{Test setup}

The schematic diagram of the test setup for CUPL2 is shown in Figure 5.17. Since the full frequency synthesizer consists of PLL components that are both monolithic and discrete, interfacing these circuits requires careful consideration of signal levels. The problems of noise and cross interference are more critical, especially at a gigahertz frequency range. Test board parasitics and transmission line effects must be considered in conjunction with device propagation delays [79]. 


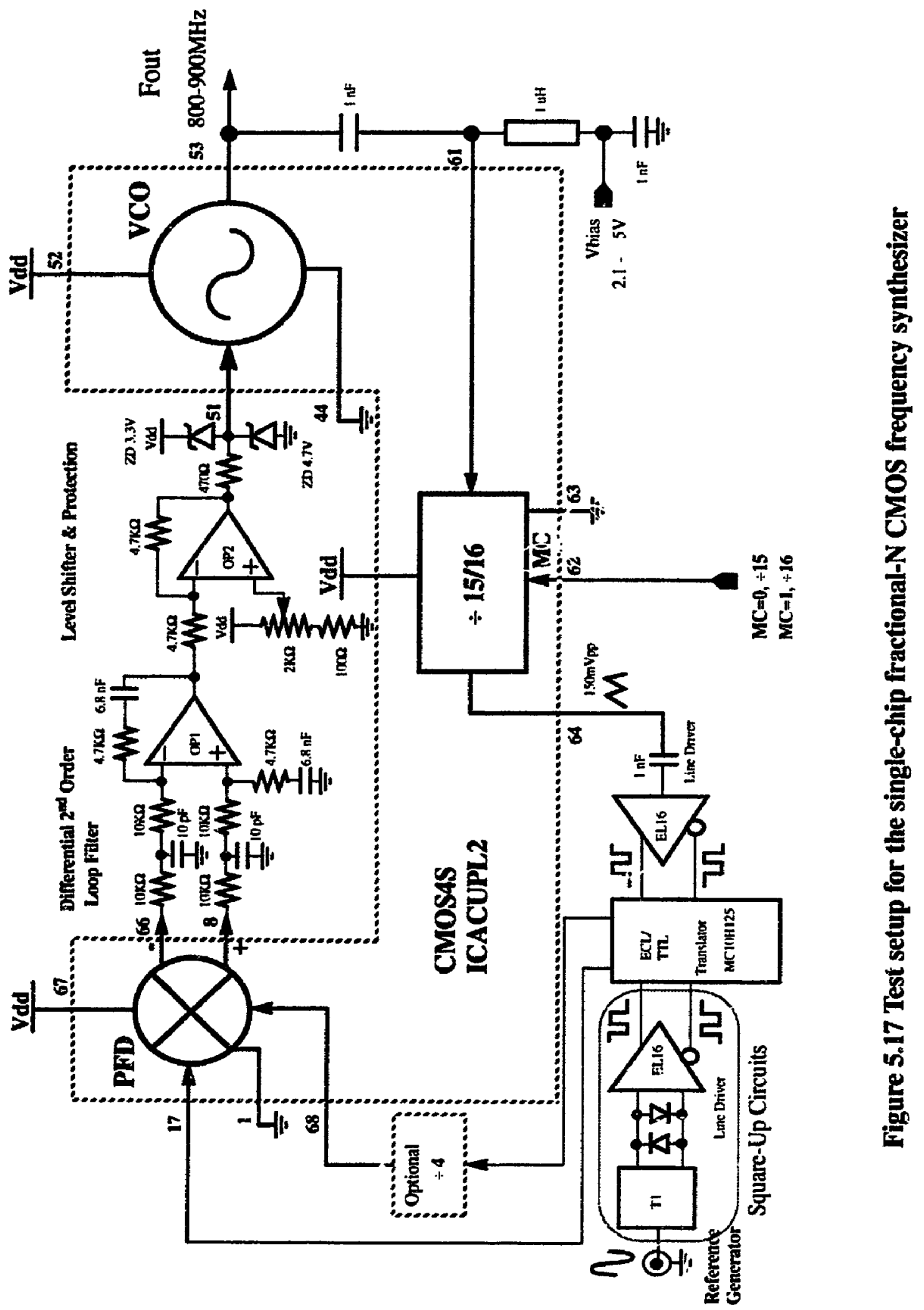


A universal two sided printed circuit board provided by the TRIO lab, one side with a ground plane, was used for the test bed. The power supply circuit (not shown in the diagram) supplied a regulated 5 volts throughout the CUPL2 chip. Decoupling capacitors of $1 \mathrm{nF}$ v'ere connected as closely as possible to the power supply pin of each circuit to decouple the high-speed switching current.

The VCO output signal was applied to the divider input via a $1-\mathrm{nF}$ coupling capacitor. A variable bias voltage was introduced at this point to level shift the ignal for proper threshold detection by the divider. The divider received the biasing from the same power supply through a variable resistor and a $1-\mu \mathrm{H}$ inductor. The inductor was necessary in order to isolate the VCO output from the power supply line.

Due to the selection of an analog, instead of a digital output pad in the divider circuit, some system modifications had to be made to facilitate the functionality of the entire chip. Since the output of the divider had insufficient drive for the PFD, two Motorola high-speed ECL line teceiver ICs, MC10EL16, and an ECL/TLL translator, MC10H125, were required. The divider output signal was buffered to an ECL level before translating back into a CMOS compatible level, which was achieved using an ECLTLL translator. To avoid level and speed mismatch another line receiver was used to buffer the reference input as well. The conditioned reference signal was also converted using an ECLTLL translator.

Input signals to the PFD can be arranged in two configurations: high reference and low reference frequencies. Without the external divide-by-4 circuit while operating the VCO $800 \mathrm{MHz}$, the reference frequencies would be higher than $50 \mathrm{MHz}$, which is considered too high for practical implementation of mobile racio terminals. As a result. the tests were performed with an external divider.

The pulse trains obtained by comparing the phase of the reference input and the divided down VCO output were differentially summed at the loop filter. The loop filter was 
constructed according to the component values determined by the loop calculations (see Section 5.2.2). External unregulated dual supplies of \pm 10 volts were required for the opamp.

An active level shifter was also implemented in order to allow a dc offset adjustment to the loop filter output. Two Zener diodes were used to clamp the VCO input to protect the VCO from being driven by excessive voltage generated from the loop filter and level shifter in the event of the PLL losing lock. These Zener diodes also help the PLL to approach lock easily when the PLL is powered up. The diodes' voltages were selected such that the voltage driving the VCO ranges between 2.2 and 4 volts. Such an arrangement centers the $\mathrm{VCO}$ at $850 \mathrm{MHz}$. The extracted dc signal from the loop filter provides VCO control input and completes the closed loop PLL.

\subsubsection{Operating range}

The functionality and performance of the fractional- $\mathbf{N}$ synthesizer were measured under various input and control conditions and are reported in this section.

A preliminary measurement was performed to ensure the proper function of the synthesizer by using a fixed divide ratio. Figure 5.18 shows the test configuration.

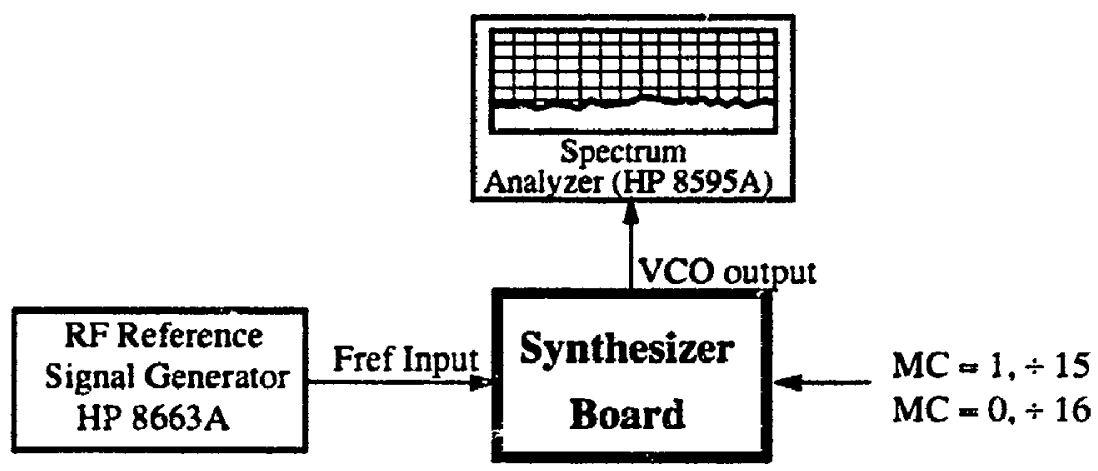

Figure 5.18 Setup configuration for measurement of synthesizer operating frequency and phase noise. 
The modulus control of the divider was first set to 1 (connected to the power line) to enable the PLL in a divide-by-15 configuration. The optional divide-by-4 was not used for this test.

A spectrum analyzer was used to check the proper operation of the frequency divider. The correct VCO output frequency in the lock condition should give Fout $=15$ Fref. By gradually increasing the reference frequency and observing the oscillating frequency, the operating range of the synthesizer was obtained. The same procedure was applied to check the synthesizer's functionality with a $\mathrm{MC}=0$, divide-by- 16 configuration. During the test, it was found that the optimum dc bias of the divider was 2.4 volts.

The synthesizer was also measured with lower reference frequencies by including a divide-by-4 circuit to provide a moduli of 60 and 64 .

Table 5.2 summarizes the results using both high and low reference frequencies.

\begin{tabular}{|c||c|c|}
\hline Configuration & $\begin{array}{c}\text { Reference input } \\
\text { Frequency (MHz) }\end{array}$ & $\begin{array}{c}\text { VCO output } \\
(\mathrm{MHz})\end{array}$ \\
\hline \hline Divide-by-15 & $53.72-57.47$ & $805.80-862.05$ \\
\hline Divide-by-16 & $50.47-53.55$ & $807.52-856.80$ \\
\hline Divide-by-60 & $13.35-14.52$ & $801.00-871.20$ \\
\hline Divide-by-64 & $12.51-13.60$ & $800.64-870.40$ \\
\hline
\end{tabular}

Table 5.2 Summarized results using different dividing ratios.

\subsubsection{Fractional division}

An attempt was made to clock the modulus control for fractional division operation. Sinze no common reference frequency between moduli 15 and 16 could be applied and still remain within the VCO tuning range, it was clear that a fractional 15/16 configuration could not be made. However, the reference ficquencies between 13.35 MHiz and 13.60 $\mathrm{MHz}$ (see Table 5.2) using a divide ratio of $60 / 64$ offered an opportunity for fractional division. 
By adding an external PAL (PAL 22V10, a programmable logic array with 10 ns propagation delay) to modify the modulus control from $15 / 16$ to $63 / 64$ (see Figure 5.19), it became possible to experiment with a new fractional division ratio.

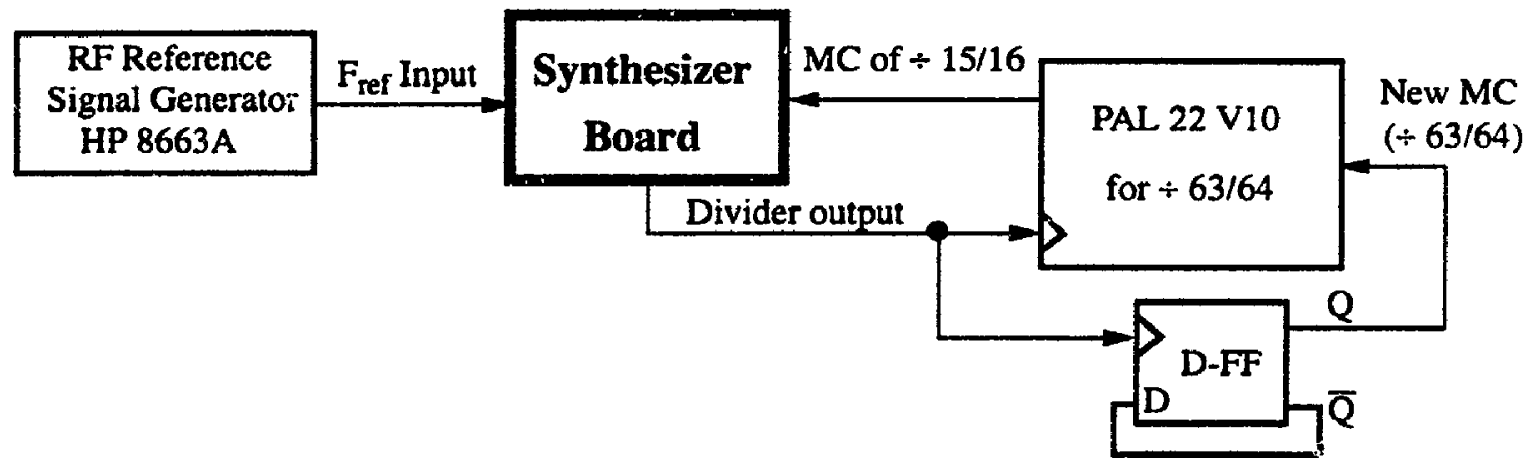

Figure 5.19 Modification for a new tractional dividing ratio.

A D flip-flop constructed in a divide-by-2 form was used to demonstrate the fractional division of the synthesizer. By taking the modified divider output to a divide-by2 , switching of the dividing ratio between $63 / 64$ was achieved.

However, the experiment showed that the lock condition under the fractional division, $63 / 64$, could not be made. It was determined that the cause was a speed mismatch problem between the external logic circuits of the PAL and the on-chip 15/16 divider.

Improyements to the fractional division loop can be achieved in two steps: (i) redesigning the divider to a higher divide ratio and, (ii) adjustment of the loop filter for optimum filtering.

\subsubsection{Synthesizer phase noise}

The closed loop PLL synthesizer spectral output was measured and recorded using a spectrum analyzer. Figure 5.20 shows one of the VCO spectral outputs measured at 850 $\mathrm{MHz}$ when the synthesizer was set at a divide-by- 60 configuration. 
Notice that the closed loop PLL produces a clean synthesized output signal near the center frequency. The two side peaks at $200 \mathrm{kHz}$ offset indicate the loop bandwidth of the PLL synthesizer. The height of these peaks is dependent upon the damping ratio of the loop filter (see Section 5.2.2).

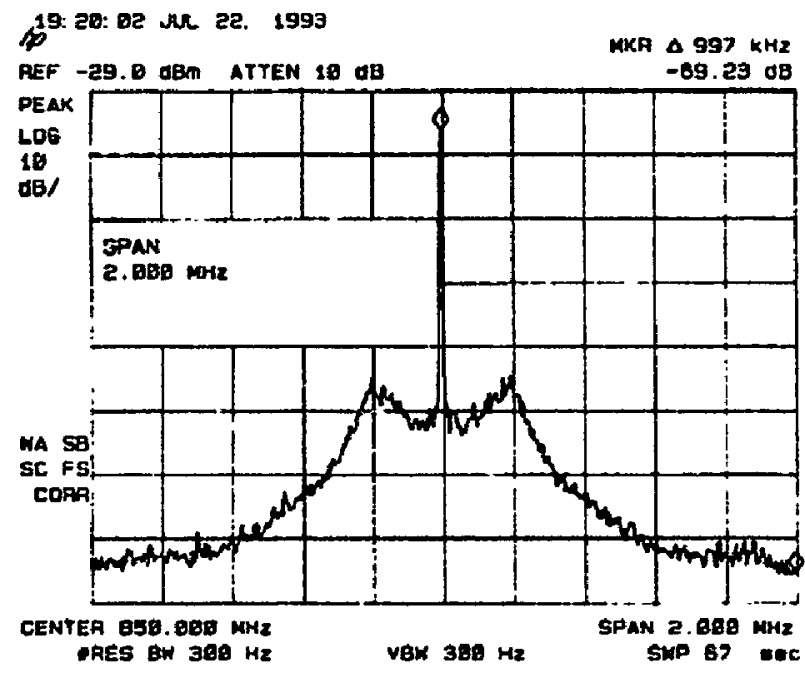

(a) 2-MHz span

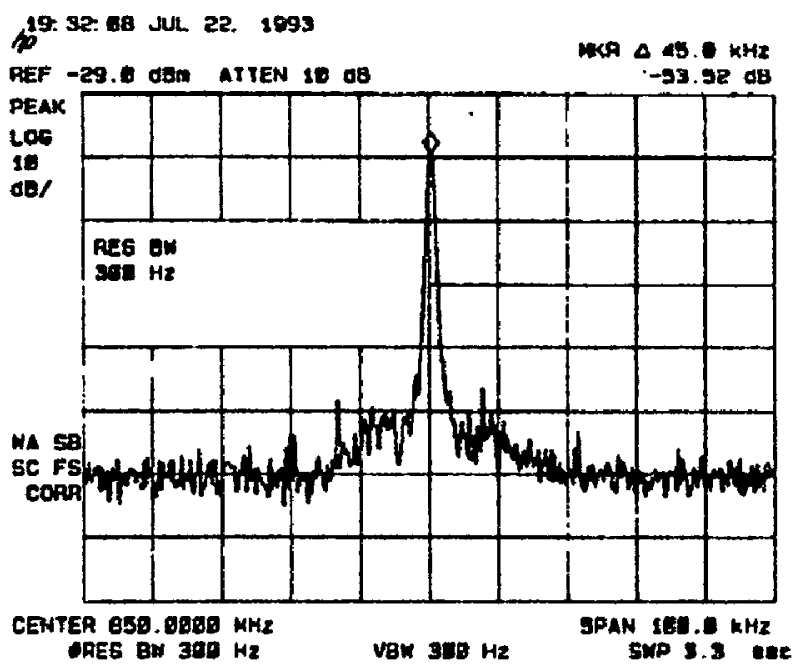

(b) 100-kHz span

Figure 5.20 PLL spectral outputs at 850-MHz center frequency.

The output phase noise at $45 \mathrm{kHz}$ and $1 \mathrm{MHz}$ offset from the carrier were measured at $-78.3 \mathrm{dBc} / \mathrm{Hz}$ and $-94.0 \mathrm{dBc} / \mathrm{Hz}$ respectively, satisfying the requirement for a cellular transmission standard [43].

To achieve better phase noise performance, the loop filter design needed to be optimized. Parameters associated with the loop filter such as lock in time, loop gain, loop bandwidth and damping, have significant effects on the overall performance of the closed loop synthesizer. However, since this research work was intended to present the experimental results on the implementation of the PLL synthesizer, the loop filter was not optimized for best performance. 
Several improvements can be made in the loop filter including the use of a higher order loop filter which can considerably help both lock in time and phase noise reduction [43].

\subsection{Summary}

The two CMOS chips consisting of three VCO structures, a no-dead-zone phasefrequency detector and a complete single-chip PLL synthesizer were tested and reported. Various tests were utilized to characterize these components and these results were then compared with the design parameters. Key VCO parameters such as the transfer characteristic, phase noise level, power consumption and sensitivity to power supply variation were evaluated. The phase detector was measured for functionality and performance.

A prototype single-chip PLL synthesizer excluding the loop filter was built and its performance evaluated. The design has proven that a gigahertz frequency synthesizer can be implemented without a submicron CMOS process. The contribution of this work is the promise it offers for a realization of a single radio IC that integrates all radio bulding blocks in a silicon chip. In this way, full advantage is taken of the high-speed performance of shorter-gate-length technologies. 


\section{Chapter 6}

\section{Conclusion}

This thesis presented a design approach for fully integrated VCOs using a pure CMOS circuit. As a result of a systematic search for proper architectures and much research on their speed limiting factors and noise, three high-speed CMOS VCOs were proposed. These circuits, through simulation and measurement, demonstrated performance and speed in a gigahertz range which was not thought possible in a conventional CMOS process.

The maximum oscillating frequency and phase noise results were superior to any previously reported CMOS VCOs. As well, thie power consumption was lower than that of any VCOs previously reported in compatible tecinologies. The proposed VCOs required no external components yet exhibited good phase noise performance comparable to LC on-chip oscillators facilitated using a high level of integration.

The innovative architectural and circuit design techniques presented in this thesis involve the use of a parasitic-capacitor-based CMOS oscillator combined with two simulation techniques developed for the characterization of the proposed VCO structures. The developed ac MOS model, although preliminary, allowed more accurate prediction of the final design, while the phase noise simulation technique helped to select the structure 
which resulted in the best phase noise performance. Consequently, previous transistoronly oscillators, having limited complexity, can now be implemented in a standard CMOS process.

The overall goal of this research was to develop a monolithic low-power frequency synthesizer for $\mathrm{GHz}$ range mobile radio applications using a non-submicron process. The realization of such a frequency synthesizer has indeed been achieved in this thesis. A total power consumption below $18 \mathrm{~mW}$, excluding the loop filter, was achieved for the CMOS components of a $900 \mathrm{MHz}$ synthesizer. The approach demonstrates the feasibility of an all CMOS frequency synthesizer for mobile radio applications. With these greater levels of integration achieved, a CMOS implementation of the synthesizer should contribute to more widespread use of integrated analog/digital components in wireless communications mass-consumer products.

Future improvernents to this implementation can be made in several ways. Clearly, a loop filter and a modulus controller, as well as its $\Delta \Sigma$ modulator noise shaping circuit for frequency programmability, could be integrated with the proposed frequency synthesizer. Moreover, other baseband-frequency components can undoubtedly be incorporated into the synthesizer in the same CMOS process. Some of the possibilities for future work also include modelling improvements for phase noise prediction using a complete Mathematica $^{T M}$ program. The preliminary MOS model presented needs to be further studied to improve accuracy. Another improvement which needs to be implemented is the application of the proper analog $1 / O$ pads or interface circuits for a gigahertz operating frequency. This will allow CMOS VCO circuits to be capable of driving other RF circuits. 


\section{Appendix A}

\section{CMOS4S Process Parameters}

This section provides information regarding mask layers, SPICE Level 3 simulation

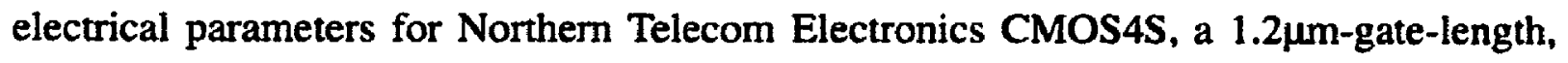
double-polysilicon, double-metal, $\mathrm{N}$-well process. They are extracted from report IC 90 01, "Design Rules and Process parameters for the Northern Telecom CMOS4S process" supported by Canadian Microelectronics Corporation.

Dimensions of the layout submitted to CMC must be specified in Design Scale Microns (DSM) using a 1.5-micron scale for the minimum feature size. The design is then scaled down by a factor of $80 \%(1.2 \mu \mathrm{m})$ in actual implementation. 


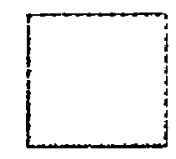

Device Well

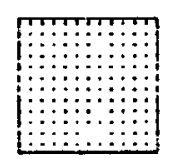

Poly

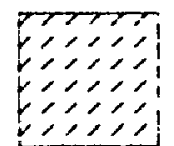

Cap-Poly

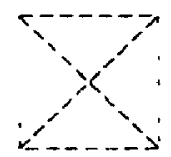

Pyrox

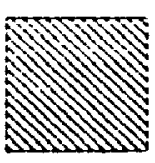

Metal1

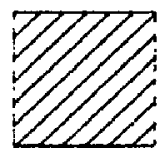

Metal2

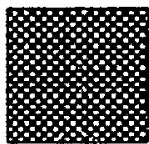

Via

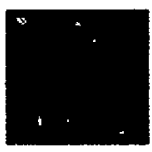

Contact

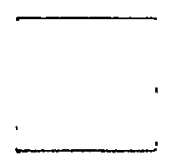

N Well

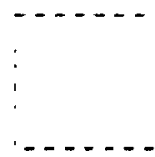

P-Guard

$\mathrm{N}+$ Exclusion

P+

Figure A.1 CN.OS4S layer legend 


\section{A.1. CMOS4S mask layers}

\begin{tabular}{|c||c|c|c|l|}
\hline $\begin{array}{c}\text { Mask } \\
\text { Level }\end{array}$ & $\begin{array}{c}\text { Name } \\
\text { (Function) }\end{array}$ & $\begin{array}{c}\text { CIF } \\
\text { Layers }\end{array}$ & $\begin{array}{c}\text { GDS II } \\
\text { Layer } \\
\text { Number }\end{array}$ & \multicolumn{1}{|c|}{ Function } \\
\hline 05 & N-Well & CNW & 1 & Defines n-wells. \\
\hline 10 & Device Well & CF & 2 & $\begin{array}{l}\text { Defines active regions. All other regions } \\
\text { will be covered by field oxide. }\end{array}$ \\
\hline 15 & $\begin{array}{c}\text { P-Guard } \\
\text { Exclusion }\end{array}$ & CPG & 3 & $\begin{array}{l}\text { Implant mask to increase p-substrate sur- } \\
\text { face doping under field oxide. Exclusion } \\
\text { mask to be drawn around n-well. }\end{array}$ \\
\hline 20 & Polysilicon & CP & 6 & $\begin{array}{l}\text { Defines polysilicor lines and transistor } \\
\text { gates. }\end{array}$ \\
\hline 27 & $\begin{array}{c}\text { Capacitor } \\
\text { Polysilicon }\end{array}$ & CCP & $:$ & $\begin{array}{l}\text { Defines top poly plates to form poly-to- } \\
\text { poly capacitors (optional). }\end{array}$ \\
\hline 30 & $\begin{array}{c}\text { N+ Doping } \\
\text { Exclusion }\end{array}$ & CNP & 7 & $\begin{array}{l}\text { Defines areas not to be doped n+. Sub- } \\
\text { strate doping will occur only in device } \\
\text { wells. }\end{array}$ \\
\hline 35 & P+Doping & CPP & 8 & $\begin{array}{l}\text { Defines areas to be doped p+. Substrate } \\
\text { doping will occur oilly in device wells. }\end{array}$ \\
\hline 40 & $\begin{array}{c}\text { Cortact } \\
\text { Window }\end{array}$ & CC & 9 & $\begin{array}{l}\text { Defines contact areas from Metal 1 to } \\
\text { polysilicon or diffusion. }\end{array}$ \\
\hline 50 & Metal 1 & CM & 10 & Defines first level metal lines. \\
\hline 60 & Via 1 & CV & 11 & $\begin{array}{l}\text { Defines contact areas from Metal 2 to } \\
\text { Metal 1. }\end{array}$ \\
\hline 70 & Metal 2 & CM2 & 12 & Defines second level metal lines. \\
\hline 95 & $\begin{array}{l}\text { Passivation } \\
\text { Windows }\end{array}$ & CG & 13 & $\begin{array}{l}\text { Defines openings in passivation layer for } \\
\text { bonding pads. }\end{array}$ \\
\hline & & &
\end{tabular}

Table A.1 CMOS4S mask layers 


\section{A.2. SPICE Level 3 Parameters}

- Nominal conditions

- All resistances (RS and RD) are for 20-micron-wide devices

\begin{tabular}{|l||l|l|c|}
\hline Parameter & \multicolumn{1}{|c|}{ NMOS } & \multicolumn{1}{c|}{ PMOS } & Units \\
\hline \hline Level & 3 & 3 & - \\
\hline UO & 566.3 & 200.8 & $\mathrm{~cm}^{2} / \mathrm{Vs}$ \\
\hline VTO & 0.7572 & -0.8307 & V \\
\hline NFS & $5.764 \mathrm{E}+11$ & $5.864 \mathrm{E}+11$ & $1 / \mathrm{cm}^{2}$ \\
\hline TPG & 1.000 & 1.000 & - \\
\hline TOX & $2.502 \mathrm{E}-8$ & $2.502 \mathrm{E}-8$ & $\mathrm{~m}$ \\
\hline NSUB & $1.400 \mathrm{E}+16$ & $2.011 \mathrm{E}+16$ & $1 / \mathrm{cm}^{3}$ \\
\hline VMAX & $1.651 \mathrm{E}+5$ & $2.823 \mathrm{E}+5$ & $\mathrm{~m} / \mathrm{s}$ \\
\hline RS & 26.77 & 58.90 & $\mathrm{ohm}$ \\
\hline RD & 26.77 & 58.90 & $\mathrm{ohm}$ \\
\hline XJ & $1.165 \mathrm{E}-07$ & $1.742 \mathrm{E}-07$ & $\mathrm{~m}$ \\
\hline LD & $1.157 \mathrm{E}-07$ & $1.364 \mathrm{E}-07$ & $\mathrm{~m}$ \\
\hline DELTA & 0.3551 & 0.4598 & - \\
\hline THETA & $6.574 \mathrm{E}-02$ & 0.1376 & $1 / \mathrm{V}$ \\
\hline ETA & $9.814 \mathrm{E}-02$ & $6.129 \mathrm{E}-02$ & - \\
\hline KAPPA & $2.000 \mathrm{E}-17$ & 7.487 & - \\
\hline PB & 0.800 & 0.800 & $\mathrm{~V}$ \\
\hline IS & $1.000 \mathrm{E}-16$ & $1.000 \mathrm{E}-16$ & $\mathrm{~A}$ \\
\hline JS & $1.000 \mathrm{E}-04$ & $1.000 \mathrm{E}-04$ & $\mathrm{~A} / \mathrm{m}^{2}$ \\
\hline CJ & $2.900 \mathrm{E}-04$ & $4.100 \mathrm{E}-04$ & $\mathrm{~F} / \mathrm{m}^{2}$ \\
\hline MJ & 0.486 & 0.540 & - \\
\hline CJSW & $3.300 \mathrm{E}-10$ & $3.400 \mathrm{E}-10$ & $\mathrm{~F} / \mathrm{m}$ \\
\hline MJSW & 0.330 & 0.300 & - \\
\hline CGSO & $1.973 \mathrm{E}-10$ & $3.284 \mathrm{E}-10$ & $\mathrm{~F} / \mathrm{m}$ \\
\hline CGDO & $1.973 \mathrm{E}-10$ & $3.284 \mathrm{E}-10$ & $\mathrm{~F} / \mathrm{m}$ \\
\hline FC & 0.500 & 0.500 & - \\
\hline
\end{tabular}

Table A.2 SPICE Level 3 parameters 


\section{A.3. Other Electrical Parameters}

\begin{tabular}{|l||l|}
\hline \multicolumn{1}{|c|}{ Resistance } & \multicolumn{1}{c|}{ ohms/sq- } \\
\hline N+ Diffusion & 45 \\
\hline $\mathrm{N}+$ Diffusion & 100 \\
\hline $\mathrm{N}+$ Poly & 30 \\
\hline P+ Poly & 30 \\
\hline $\mathrm{N}$-well & $1.8 \mathrm{~K}$ (geometry and voltage dependent) \\
\hline Metall & 0.050 \\
\hline Metal2 & 0.025 \\
\hline Metall - Diffusion Contacts & $20 \mathrm{ohms}(1.2 \times 1.2 \text { microns })^{*}$ \\
\hline Metall - Poly Contacts & 10 ohms $(1.2 \times 1.2 \text { microns })^{*}$ \\
\hline Metall - Metal2 Vias & 0.120 ohms $(1.6 \times 1.6$ microns) * \\
\hline
\end{tabular}

Table A.3 Resistance

\begin{tabular}{|l||c|c|}
\hline \multicolumn{1}{|c||}{ Capacitance } & Area Component $\left.\left(\mathrm{pF} / \mu^{2}\right)^{2}\right)$ & Edge Component $(\mathrm{pF} / \mu \mathrm{m})$ \\
\hline Metal1 - Field & $2.9 \mathrm{E}-5$ & $5.00 \mathrm{E}-5$ \\
\hline Metal1 - Poly & $6.0 \mathrm{E}-5$ & \\
\hline Metal1 - Diffusion & $5.5 \mathrm{E}-5$ & \\
\hline Poly - Field & $6.4 \mathrm{E}-5$ & $2.20 \mathrm{E}-5$ \\
\hline Metal2 - Field & $1.6 \mathrm{E}-5$ & $4.63 \mathrm{E}-5$ \\
\hline Metal2 - Poly & $2.5 \mathrm{E}-5$ & \\
\hline Metal2 - Metal1 & $2.0 \mathrm{E}-5$ & \\
\hline Metall - Field & $5.2 \mathrm{E}-5$ & \\
\hline Capacitor Poly to Poly & $7.76 \mathrm{E}-4$ & $1.06 \mathrm{E}-4$ \\
\hline
\end{tabular}

Table A.4 Capacíance

* Physical dimensions, not design scale dimensions 


\section{Appendix B}

\section{Physical Design: \\ Chip Configurations, I ayouts, and Device Sizes}

The implemented layouts for this thesis work are presented here. CUPLL and CUPL2 chip configurations and their pin assignments are giver along with their actual chip outlines. The detailed layout created for each proposed design is presented, followed by the transistor widths (actual sizes in $\mu \mathrm{m}$ ).

- CUPLl

VCO1, VCO2, VCO3 and No-Dead-Zone phase-frequency detector (PFD) are the included cells on CUPLL. The PFD detailed design can be found in [62]. All transistors in each circuit are constructed using a minimum gate-length of $1.2 \mu \mathrm{m}$.

- CUPL2

This contains multi-project designs; the major layouts created for this thesis work are modified versions of $\mathrm{VCO} 1$ and PFD. The 1.4-GHz frequency divider integrated on the chip is the layout work of N. Foroudi [5]. 
ICACUPLL VCOs and PFD

Pin Arrangement and Description

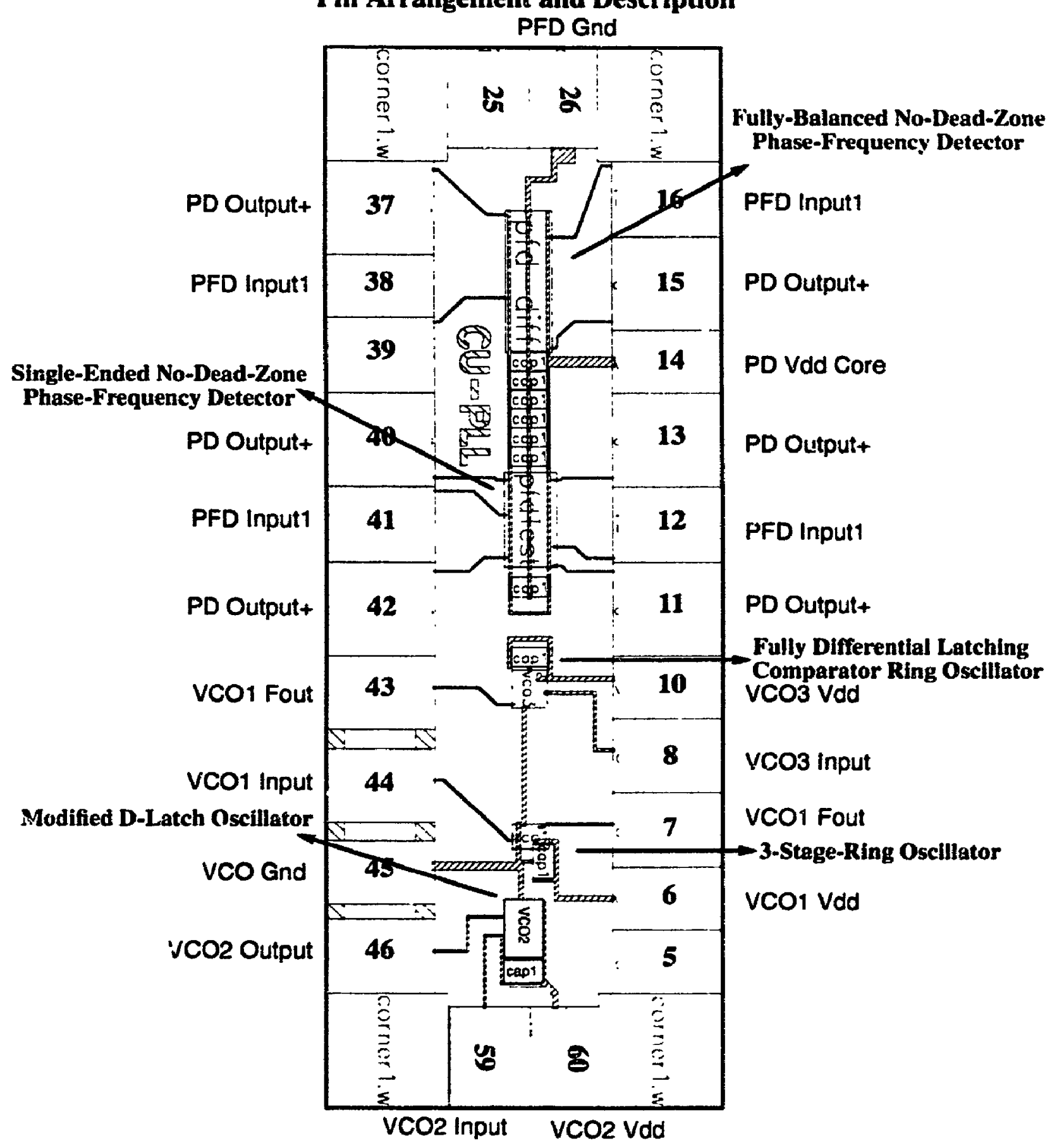

Figure B.I CUPLL. Pin assignments and descriptions. 
ICACUPL2 Frequency Synthesizer Chip Pin Arrangement and Description

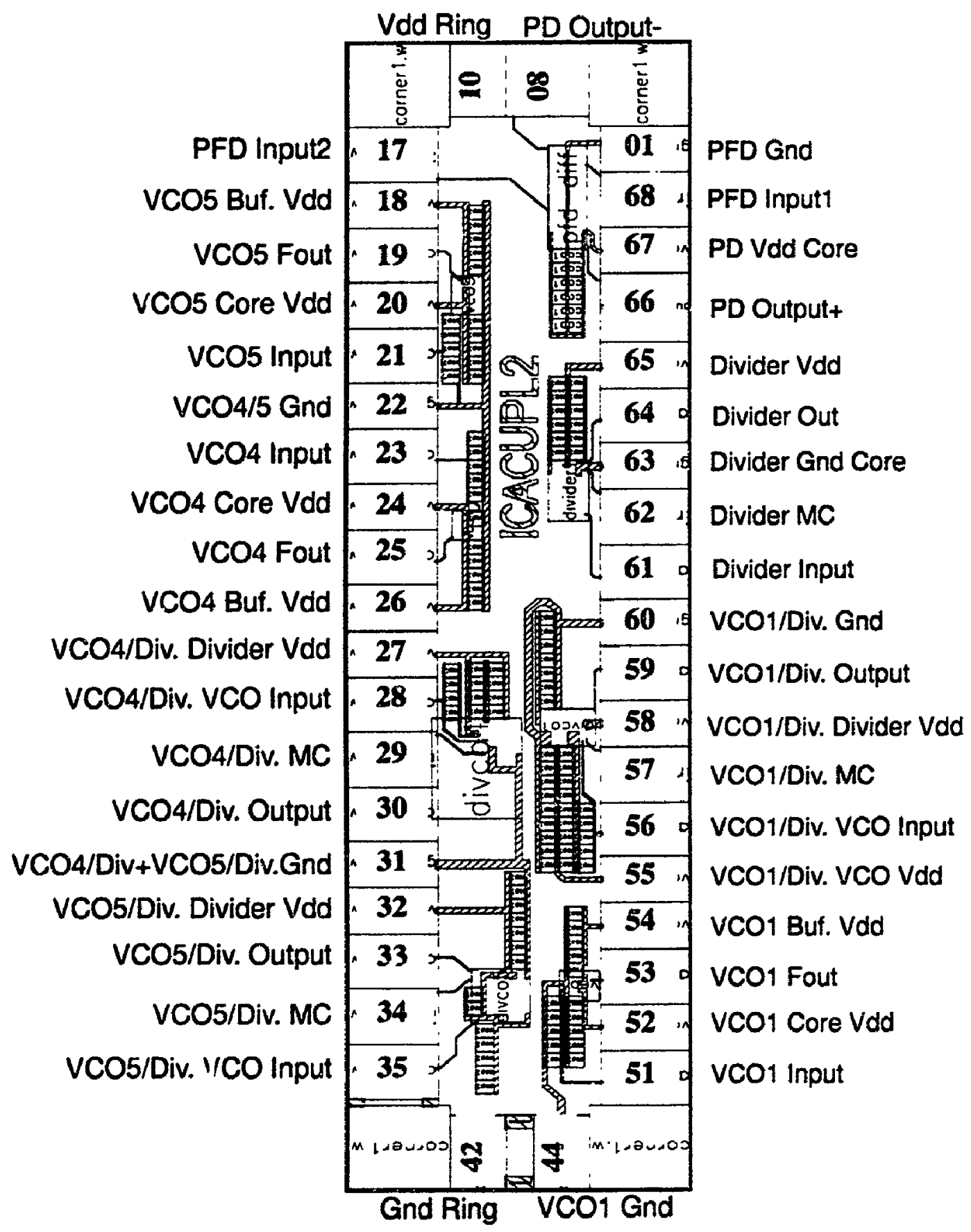

Figure B.2 CUPL2. Pin assignments and descriptions. 


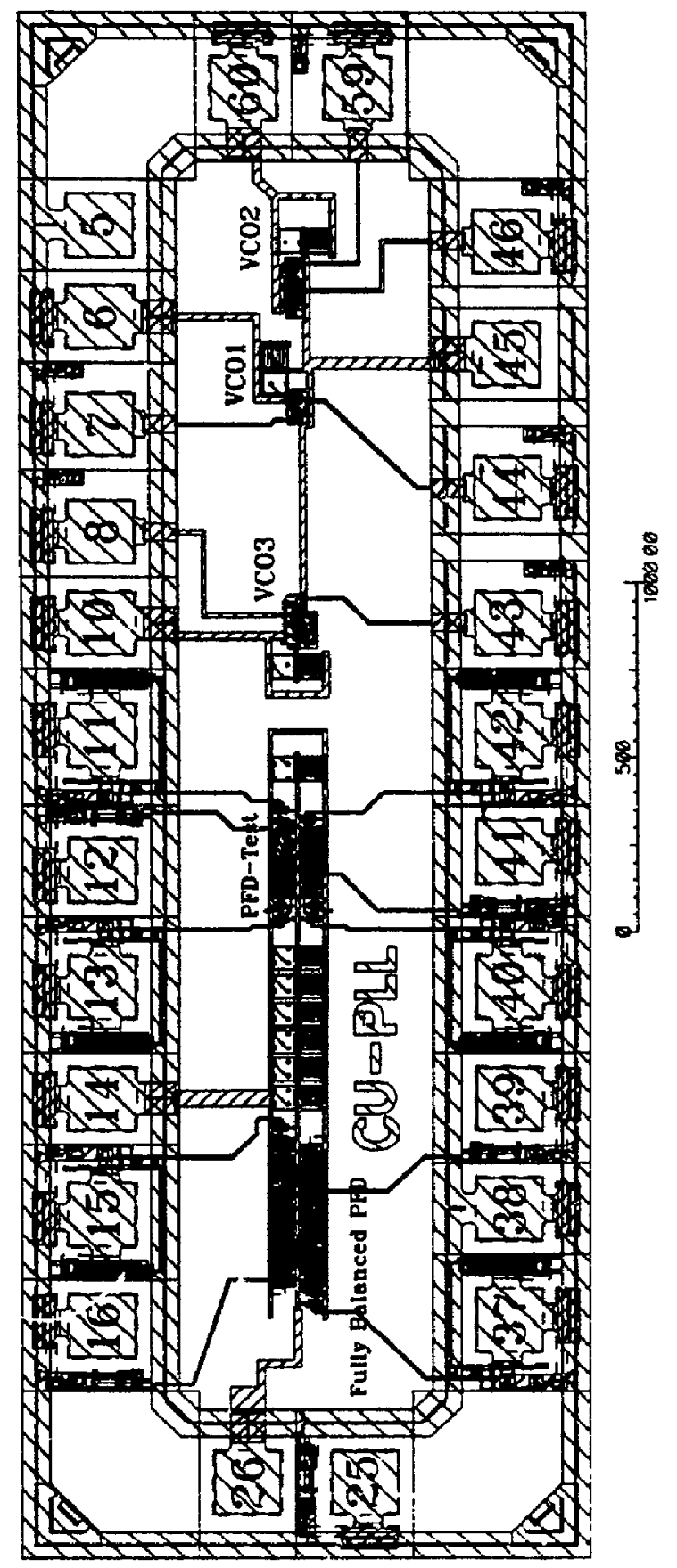

Figure B.3 CUPLL, outlined fabricated chip. 


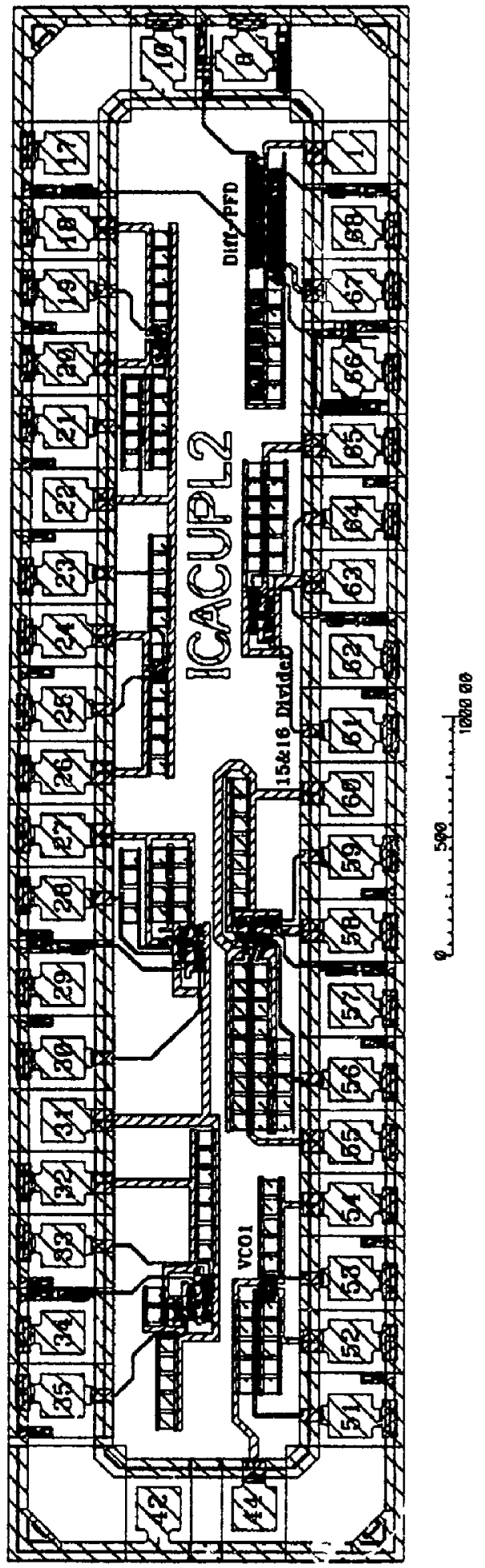

Figure B.4 CUPL2 (ICACUPL2), outlined fabricated chip. 

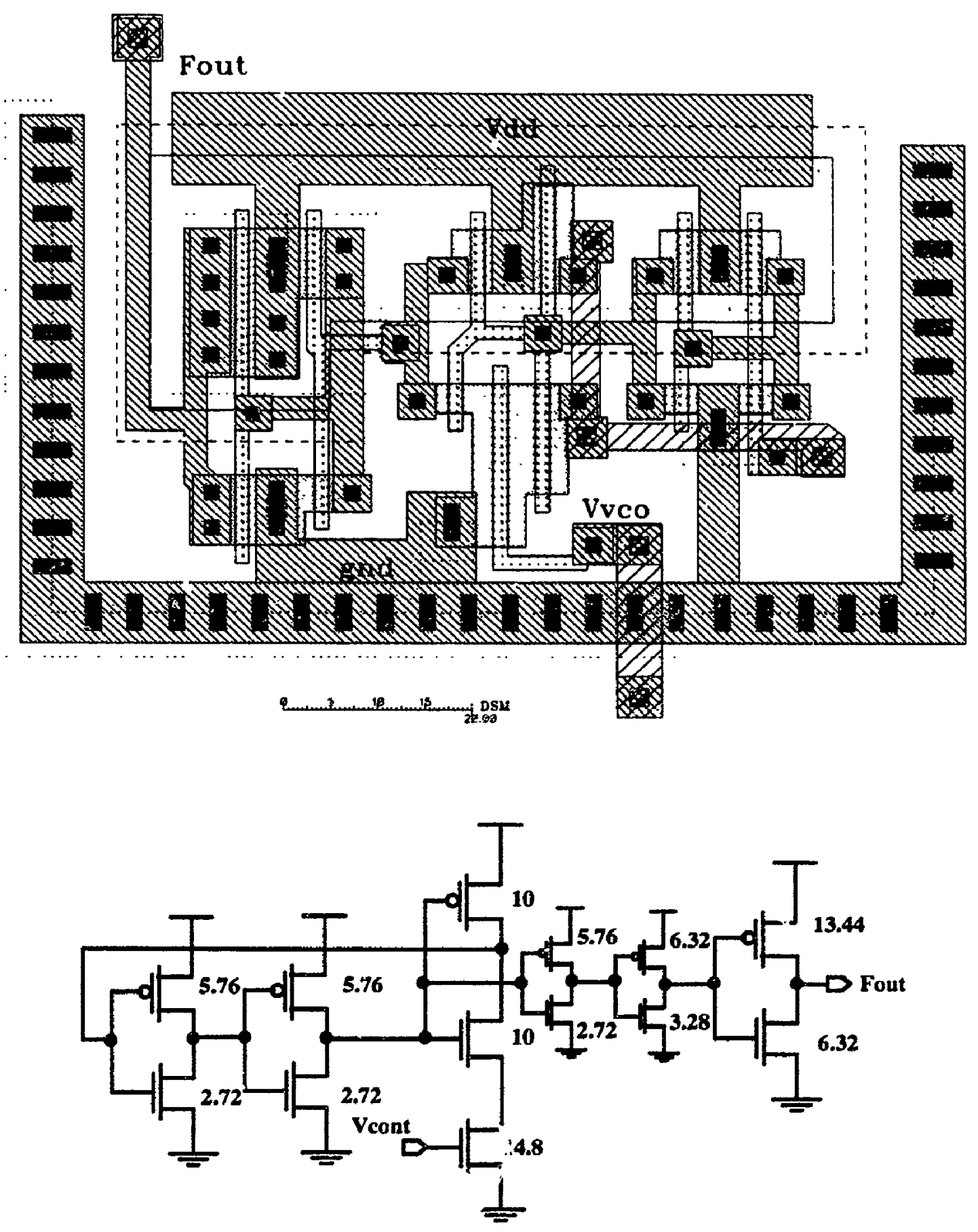

Figure B.5 VCO1, three-stage modified ring oscillator. 


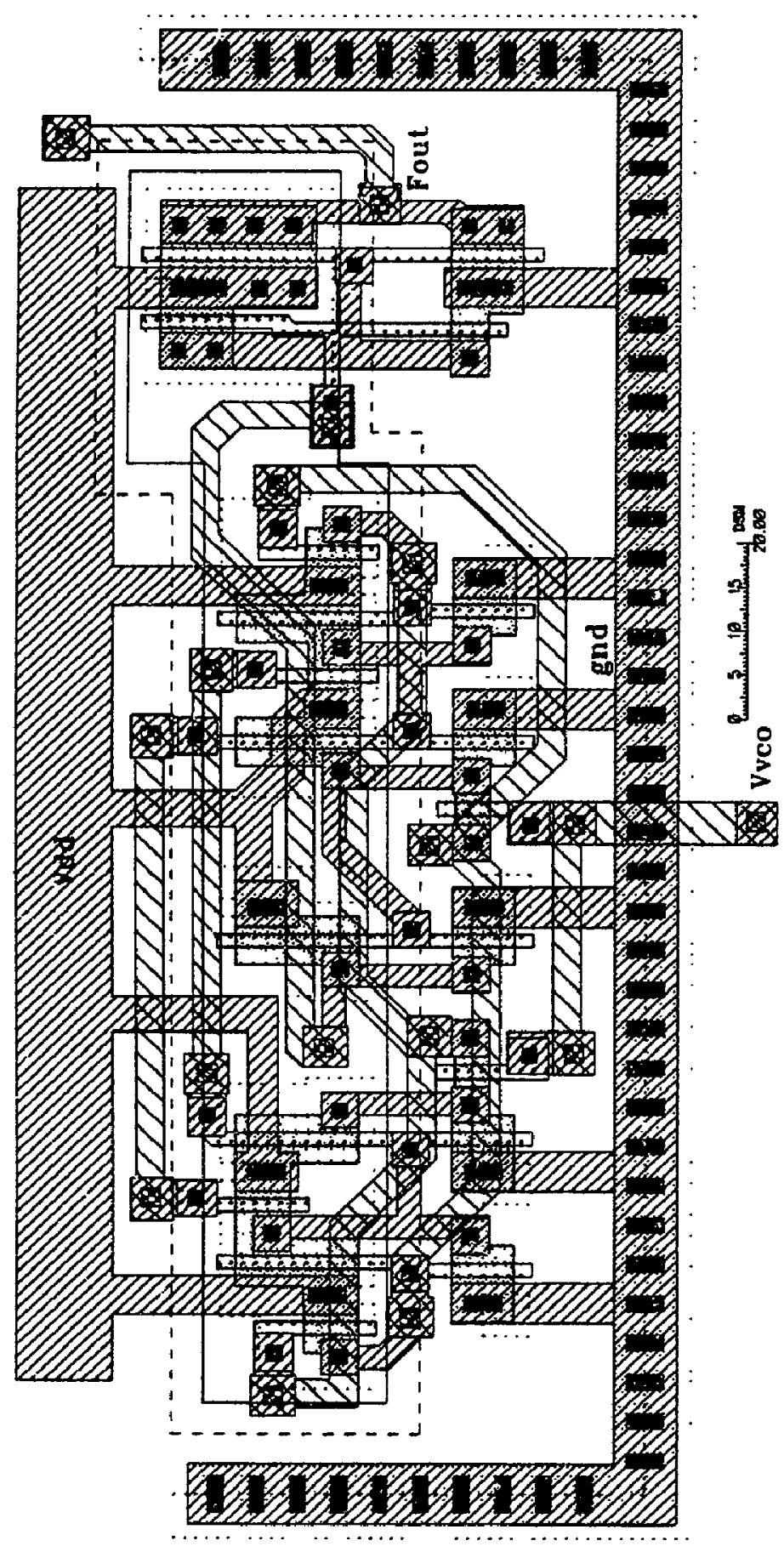

Figure B.6 VCO2, modified D-latch ring oscillator. 


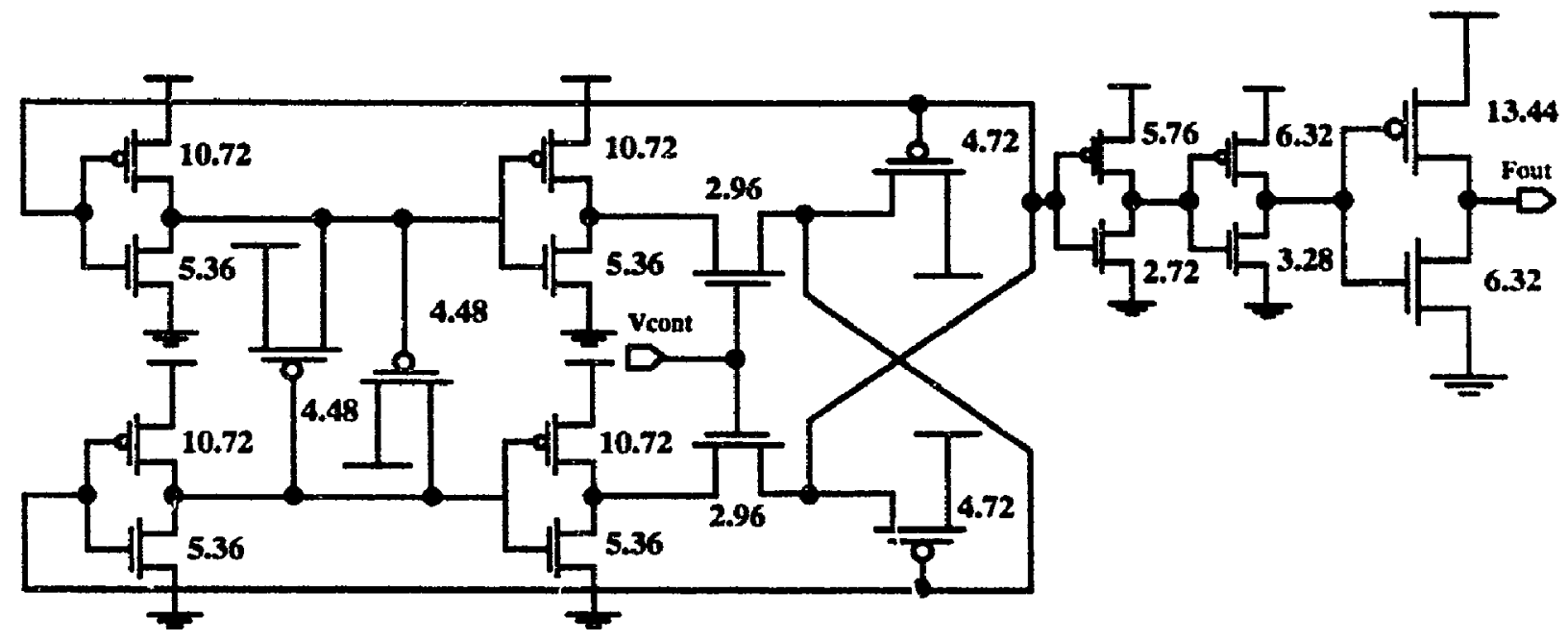

Figure B.7 VCO2 transistor sizes. 


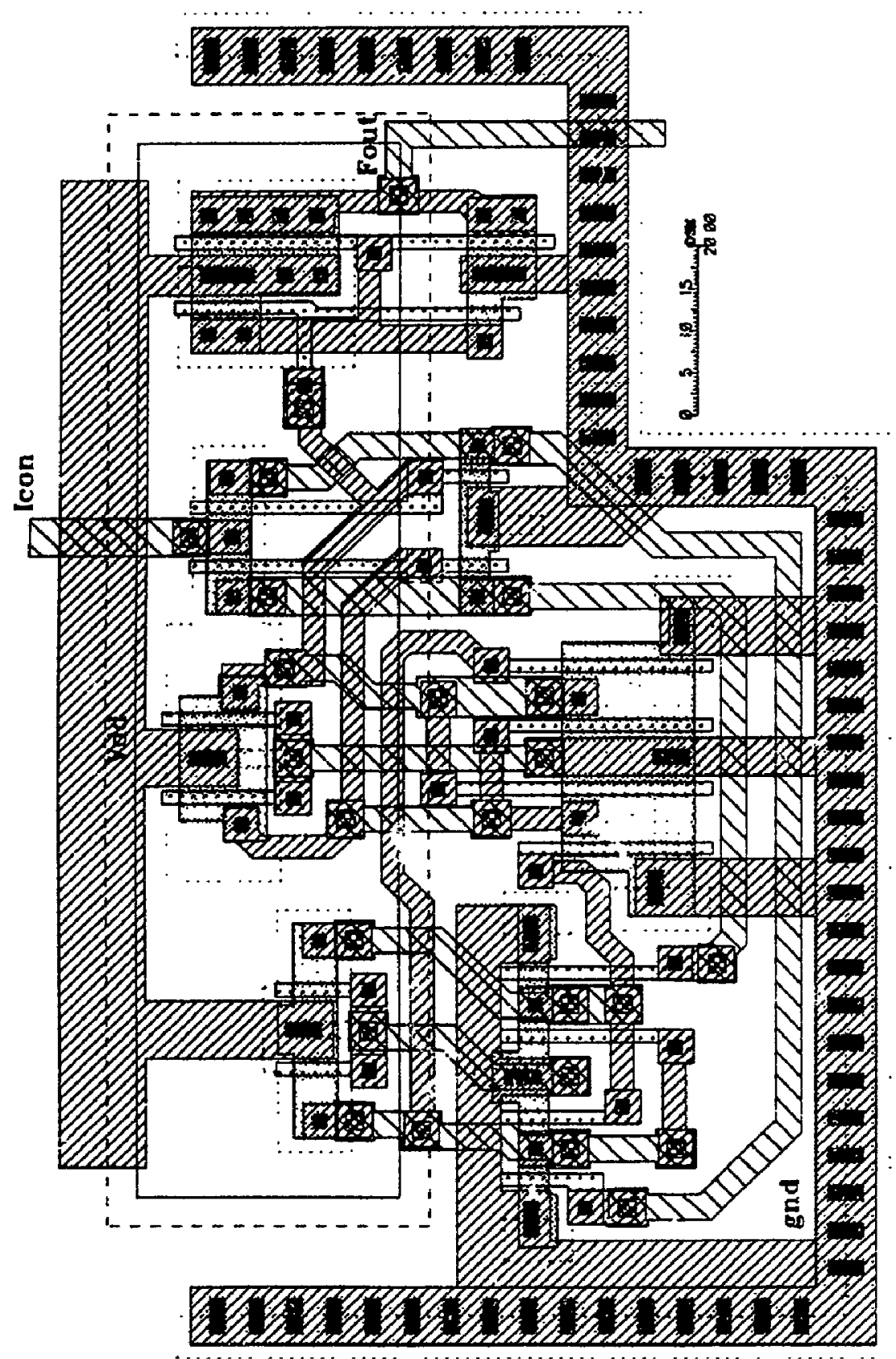

Figure B.8 VCO3, fully differential ring oscillator. 


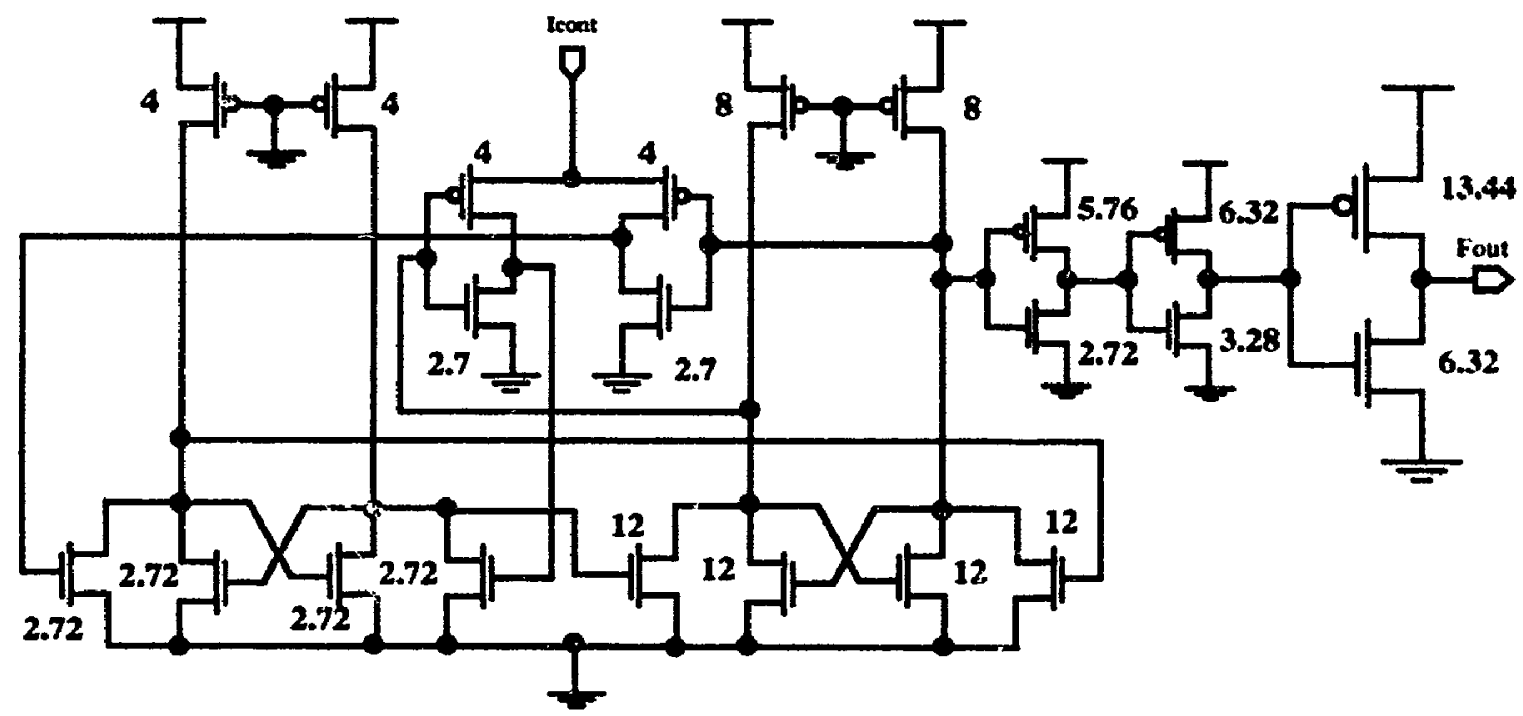

Figure B.9 VCO3, transistor sizes. 


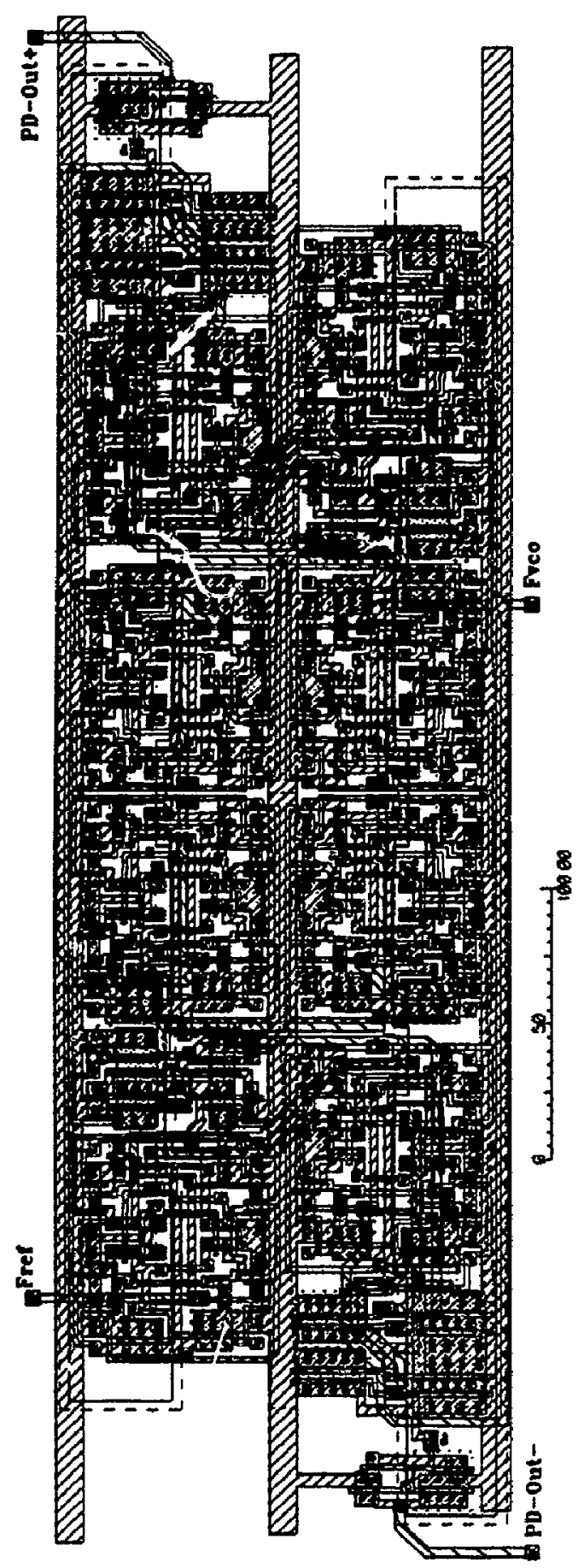

Figure B.10 Fully balanced phase-frequency detector with no dead zone. 


\section{References}

[1] L. Fang et ai., "A 700-MHz 24-b Pipelined Accumulator in 1.2- $\mu \mathrm{m}$ CMOS for Application as a Numerically Controlled Oscillator," IEEE Jour. Solid-State Ccts., Vol. 28, No. 8, pp. 878-886, August, 1993.

[2] H. T. Nicholas and H. Samueli, "A 150-MHz Direct Digital Frequency Synthesizer in 1.25- $\mu \mathrm{m}$ CMOS with -90 dBc Spurious Performance," IEEE Jour. Solid-State Ccts., Vol. 26, No. 12, pp. 1959-1969, December, 1991.

[3] M. Soyuer and R.G. Meyer, "High-Frequency Phase-Locked Loops in Monolithic Bipolar Technology," IEEE Jour. Solid-State Ccts., Vol. 24, pp. 787-795, 1989.

[4] Motorola, "High Performance Frequency Control Products," Product Catalogue 1993.

[5] N. Foroudi, “A High-Speed CMOS Dual-Modulus Frequency Divider for Mobile Radio Frequency Synthesizers," M. Eng. Thesis, Carleton University, Ottawa, Canada, 1991.

[6] T. Pantazopoulos, "A Digital Phase-Frequency Detector with no Dead-Zone," Course Project 97.589F, ASICs in Telecommunicaticr.s, 1992.

[7] T. Riley, M. Copeland, and T. Kwasniewski, "A Deita-Sigma Modulator Fractional-N Synthesizer," IEEE Jour. Solid-State Ccts., Vol. 28, No. 5, pp. 553-559, May, 1993.

[8] T. Kenny, "Components and Architectures for an Integrated Wideband FM Receiver," M. Eng. Thesis, Carleton University, Ottawa, Canada, 1993.

[9] R. S. Soin, F. Maloberti and J. Franca, "Anaiogue-Digital ASICs, circuit techniques, design tools and application," Peter Peregrinus, 1991. 
[10] Milt Leonard, "Communications Terminals Get Personal," Electronic Design, pp. 6167, February 1993.

[11] J. Smith, "Modern Communication Circuits 1 ${ }^{\text {st }}$ edition," McGraw-Hill, 1986.

[12] W.F. Egan, "Frequency Synthesis by Phase Lock," Wiley, 1981.

[13] B. Goldberg and H. Eisenson, "Frequency Synthesizer Strategies for Wireless," Microwave Journal., pp. 24-40, June, 1993.

[14] W. C. Beam, "Fast-Switching Synthesizers Track Agile Signals," Microwave and RF Journal, pp. 117-124, September, 1991.

[15] A. Gilmore and R. Komfeld, "Hybrid PLLDDS Frequency Synthesizers," RF Design, pp. 41-45, July, 1990.

[16] V. Manassewitch, "Frequency Synthesizers Theory and Design," Wiley, 1976.

[17] I. Jaquet, "A Comparison of Phase Noise Control Techniques Used in High-Speed Frequency Synthesizers for Mobile Radio Transceivers," M. Eng. Thesis, Carleton University, Ottawa, Canada, 1990.

[18] B. Lai and R.Walker, "A Monolithic $622 \mathrm{Mb} / \mathrm{s}$ Clock Extraction Data Retiming Circuit," IEEE Int'l Solid-State Ccts. Conf., pp. 144-145, 1991.

[19] L. Devito et al.. "A $52 \mathrm{MHz}$ and $155 \mathrm{MHz}$ Clock-Recovery PLL," IEEE Int"! SolidState Ccts. Conf., pp. 142-143, 1991.

[20] H. Lee and J. F. Bulzacchelli, "A $155 \mathrm{MHz}$ Clock Recovery Delay-and Phase Locked Loop," IEEE Int'l Solid-State Ccts. Conf., pp. 160-161, 1992.

[21] K. Washio et al., "An All-Band TV Tuner IC with 10-GHz/100-V Mixed Analog/Digital Si Bipolar Technology," IEEE Jour. Solid-State Ccts., Vol. 27, No.9, pp. 12641268, September, 1992.

[22] R.Walker et al., "A 2-Chip 1.5Gb/S Bus-Oriented Serial Link Interface," IEEE Int"l Solid-State Ccts. Conf., pp. 226-227, 1992. 
[23] M. Soyuer and H. A. Alinspan, "A Monolithic $2.3 \mathrm{~Gb} / \mathrm{s} 100 \mathrm{~mW}$ Clock and Dati Recovery Circuit," IEEE Int'l Solid-State Ccts. Conf., pp. 158-159، 1993.

[24] K. Kato et al., "A Low-Power 128-Mhz VCO for Monolithic PLL IC's," IEEE Jour. Solid-State Ccts., Vol. 23, pp. 474-479, April, 1988.

[25] Kurata et al., "PLL-Based BiCMOS On-Chip Clock Generator for Very High-Speed Microprocessor," IEEE Jour. Solid-State Ccts., Vol. 26, No. 4, pp. 585-589. April, 1991.

[26] K. Huehne et al., "A Single-Chip, 1.2GHz PLL Frequency Synthesizer Using Reduced Capacitance, Dual Gate, BiCMOS Technology," IEEE Custom IC. Conf. pp. 24.3.1-24.3.5, 1992.

[27] B. Thompson et al., "A $300 \mathrm{MHz}$ BiCMOS Seriai Data Transceiver." IEEE Custom IC. Conf., pp. 16.7.1-16.7.5, 1993.

[28] H. Rasijn and P. O'Connor, "A PLL-Based 2.5-Gb/s GaAs Clock and Data Regenerator IC," IEEE Jour. Solid-State Ccts., Vol. 26, pp. 1345-1352, 1991.

[29] F. Naber et al., "A Fast-Settling GaAs-Enhanced Frequency Synthesizer," IEEE Jour. Solid-State Ccts., Vol. 27, No. 10, pp. 1327-1330, 1992.

[30] Y. Yamauchi et al., "A 15-GHz Monolithic Low-Phase Noise VCO Using AlGaAs/ GaAs HBT Technology," IEEE Jour. Solid-State Ccts., Vol. 27, No. 9, pp. 14441447. October, 1992.

[31] T. Yamada et al., "A 1.2 GHz Single-Chip NMOS PLL," IEEE Int'l Solid-Siate Cits. Conf., pp. 24-25, 1985.

[32] 5. Khursheed and A. Abidi, "NMOS 10"s for Clock and Data Regeneration in Gigabitper-Second Optical-Fiber Receivers," IEEE Jour. Solid-State Ccts., Vol. 27. pp. 17631774, December, 1992.

[33] R.F. Bitting and W.P. Repasky, "A 30-128 MHz Frequency Synthesizer Standard Cell." IEEE Custom IC. Conf., pp. 24.1.1-24.1.6, 1992. 
[34] R. Shariatdoust et al., "A Low Jitter 5 tc $180 \mathrm{MHz}$ Clock Synthesizer for Video Graphics," IEEE Custom IC. Conf., pp. 24.2.1-24.2.5, 1992.

[35] 1.A. Young et al., "A PLL Clock Generator with 5 to $110 \mathrm{MHz}$ of Lock Range for Microprocessors," IEEE Jour. Solid-State Ccts., Vol. 27, No. 11, pp. 1599-1606, November, 1992.

[36] M. Banu and A. Dunlop, "A $660 \mathrm{Mb} / \mathrm{s}$ CMOS Clock Recovery Circuit with Instantaneous Locking for NRZ Data and Burst-Mode Transmission," IEEE Int'l Solid-State Ccts. Conf., pp. 102-103, 1993.

[37] D. L. Chan and R. Waldron, "A Single-chip $266 \mathrm{Mb} / \mathrm{s}$ CMOS transmitter/Receiver for Serial Data Communications," IEEE Int'l Solid-State Ccts. Conf., pp. 100-101, 1993.

[38] M. Horowitz et al., "PLL design for a $500 \mathrm{Mb} / \mathrm{s}$ Interface," IEEE Int'l Solid-State Ccts. Conf.. pp. 160-161, 1993.

[39] ClCC'93 Panel Discussion, "Personal Communications In The Next Decade: Which Technologies Will You be Carrying In your Pocket?," IEEE Custom iC. Conf., Session 19, 1993.

[40] H. Samueli et al., "Hardware Technologies for Robust Personal Communication Transceivers; Semi-Annual Technical Report, January, 1993," Integrated Circuits \& Systems Laboratory, Electrical Engineering Department, University of California, Los Angeles.

[41] N. Camilleri et al., "Silicon MOSFETs, The Microwave Device Technology for the 90's." IEEE Microwave and Millimeter-Wave Monolithic Circuits Symposium, pp. 545-548, 1993.

[42] J.G. Sneep and C.J.M. Verhoeven, "A New Low-Noise 100-MHz Balanced Relaxation Oscillator," IEEE Jour. Solid-State Ccts., Vol. 25, No. 3, pp. 692-698, June, 1990.

[43] Electronic Industrial Association, "Cellular System Dual-Mode Mobile Base StationBase Station Compatibility Standard," EIA/TIA/IS-54-A, March 1991.

[44] D.H. Wolaver, "Phase-Locked Loop Circuit Design," Prentice-Hall, 1991. 
[45] N.M. Nguyen and R.G. Meyer, "A 1.8GHz Monolithic LC Voltage-Controlled Oscillator," IEEE Int'l Solid-State Ccts. Conf., pp. 158-159, 1992.

[46] A.A. Abidi and R.G. Meyer, "Noise In Relaxation Oscillators," IEEE Jour. Solid-State" Ccts., Vol. SC-18, No. 6, pp. 794-802, April, 1983.

[47] J. T. Wu, "A Bipolar 1 GHz Multi-Decade Monolithic Variable-Frequency Oscillatcr." IEEE Int'l Solid-State Ccts. Conj., pp. 106-107, 1990.

[48] C.J.M. Verhoeven, "A High-Frequency Electronically Tunable Quadrature Gscillator," IEEE Jour. Solid-State Ccts., Vol. 27, No. 7, pp. 1097-1100, July, 1992.

[49] T.P. Liu and R.G. Meyer, "A 250MHz Monolithic Voltage Controlled Oscillator," IEEE Int'l Solid-State Ccts. Conf., pp. 21-22, 1988.

[50] Fujitsu, “MB551," Data Sheet, Fujitsu Microelectronics, Inc., June 1991.

[51] M. Shigaki et al., "GaAs Monolithic Astable Multivibrator Type VCO operable up to KU Band," IEEE Trans. Electron Devices., pp. 251-256, 1992.

[52] M. Banu, "MOS Oscillatcrs with Multi-Decade Tuning Range and Gigahertz Maximum Speed," IEEE Jour. Solid-State Ccts., Vol. 23, pp. 1386̈-1393, December, 1988.

[53] S.K. Enam and A.A. Abidi, "A 300-MHz CMOS Voltage-Controlled Ring Oscillator," IEEE Jour. Solid-State Ccts., Vol. 25, No. 1, pp. 312-314, February, 1990.

[54] M. H. Wakayama and A.A. Abidi, "A 30-MHz Low-Jitter High Linearity CMOS Voltage-Cuntrolled Oscillator," IEEE Jour. Solid-State Ccts., Vol. SC-22, No. 26, pp. 1074-1081, December, 1987.

[55] M.P. Flynn and S.U. Lidholm, "A 1.2- $\mu \mathrm{m}$ CMOS Current-Controlled Oscillator," IEEE Jour. Solid-State Ccts, Vol. 27, No. 7, pp. 982-987, July, 1992.

[56] M. Soyuer and J.D. Warnock, "Multigigahertz Voltage-Controlled Oscillators in Advanced Silicon Bipolar Technology," IEEE Jour. Solid-State Ccts., Vol.27, pp. 668 670, April, 1992. 
[57] A. B. Grebene, "Bipolar and MOS Analog Integrated Circuit Design," John Wiley \& Sons, 1984, Chapter 11.

[58] J. Danvidse, "Analog Electronic Circuit Design," Prentice-Hall, 1991.

[59] J.P. Uyemura, "Circuit Design for CMOS VLSI," Kluwer Academic Publishers, 1992, Ch. 2, 3,6, 7 .

[60] A. S. Sedra and K. C. Smith, "Microelectronic Circuits," Third Edition, Saunders College Publishing, Ch. 13.

[61] A. Itill and J. Surber, "The PLL Dead Zone and How to Avoid it," RF Design., pp. 131-134, March, 1992.

[62] M. Thamsirianunt, "Design of Linear Digital Phase-Frequency Detector," Course Project 97.589X, High Performance BiCMOS Design, 1993.

[63] J. C. Crawford, "Synthesizer Designs Minimize Phase Noise in Cellular Systems," Microwave and RF Journal, pp. 69-78, January, 1993.

[64] C. Plett, "A Study of Continuous-Time Filters," Ph.D. Thesis, Carleton University, Ottawa, Canada, 1991.

[65] S. Goldman, "Differential Circuit Minimizes Detector Distortion in PLLs," Microwaves \& RF, pp. 145-151, May, 1992.

[66] R.S. Co et al., "A Differential PLL Archit. ture for High Speed Data Recovery," IEEE Custom IC. Conf., pp. 7.2.1-7.2.4, 1988.

[67] H. I. Cong et al, "Multigigahertz CMOS Dual-Modulus Prescaler IC," IEEE Jour. Solid-State Ccts., Vol. 23, pp. 1189-1194, Oct., 1989.

[68] M. J. Underhill, "Fundamentals of Oscillator Perfonuance," IEE Electronics and Communication Engineering Journal, pp. 1_.. ..\$3, 1992.

[69] NOLAL User's Manual, Macallan Consulting, 1992. 
[70] S. Wolfram, "Mathematica," $2^{\text {nd }}$ edition., Addison-Wesley Publishing Company, Inc., 1991.

[71] Hspice User's Manual Vol. 3, "Analysis and Methods," Meta-Software, Inc.. 1992.

[72] Y. P. Tsividis, "Operational and Modeling of the MOS Transistor," McGraw-Hill, 1988.

[73] H. Hu and P. G. Grey, "A Monolithic $480 \mathrm{Mb} / \mathrm{s}$ Parallel AGC/Dicision/Clock-Recovery Circuit in 1.2um CMOS," IEEE Int'l Solid-State Ccts. Conf., pp. 98-99, 1993.

[74] F. L. Martin, “A BiCMOS 50-MHz Voltage-Controlled Oscillator with Quadrature Outputs," IEEE Custom IC. Conf., pp. 27.4.1-27.4.4, 1993.

[75] M. Banu, "Design of High-Speed, Wide-Band MOS Oscillators for Monolithic PhaseLocked Loop Applications," IEEE Custom IC. Conf., pp. 1673-1677, 1988.

[76] Eldo, User's Manual, V.4.1.X, "Electrical Circuit Simulator; Chapter 7 Post Processor XELGA," ANACAD Computer System, July 1992.

[77] CPGA Package, Electrical/Thermal Specification, Published by NTE Packaging Technology Group, February, 1989.

[78] Hewlett Packard, User's Manual, "HP 3048A Phase Noise Measurement System," 1989.

[79] I. Poole, "Synthesizer Noise," Electronics World \& Wireless World, pp. 820-825, September, 1990. 


\section{Bibliography}

1 M. Thamsirianunt and T. A Kwasniewski, “A $1.2 \mu \mathrm{m}$ CMOS Implementation of a Low- Power 900-MHz Mobile Radio Frequency Synthesizer," to be published.

2 D. Rabaey et al., "Technology Constraints in the VLSI Implementation of Digital Mobile Radio Terminal," Electrical Communication, Vol. 65, No. 2, 1993.

3 H. Pohjonen and M. Andersson, "Circuit Optimization as a Tool for The Design of 1.02.0 GHz CMOS Topologies," IEEE Transactions on Circuits and Systems, pp. 21762179,1991 .

4 J.R. Long, "High Frequency Integrated Circuit Design in BiCMOS for Monolithic Timing Recovery,” M. Eng. Thesis, Carleton University, Ottawa, Canada, 1992.

5 P.H. Saul and D.G. Taylor, "A High Speed Direct Frequency Synthesizer," IEEE Jour. Solid-State Ccts., Vol. 25, No. 1, pp. 215-219, February 1990.

6 R.M. Herman et al., "A GPS Receiver with Synthesized Local Oscillator," IEEE Int'l Solid-State Ccts. Conf., pp. 194-195, 1989.

7 R. Schaumann, M. S. Ghausi and K. R. Laker, "Design of Analog Filters, Passive, Active RC and Switched Capacitor," Prentice Hall, 1990.

8 P. R. Grey and R. G. Meyer, "Analysis and Design of Analog Integrated Cicuits," John Wiley \& Sons, 1993. 
9 A. V. Oppenheim and R. W. Schafer, "Discrete-Time Signal Processing." Prentice Hall, 1989.

10 Z. Wang, "Multigigahertz Varactorless Si Bipolar VCO IC," Electronics Letters, Vol. 28, No. 6, pp. 548-549, 12th March, 1992.

11 N. Weste and K. Eshraghian, "Principle of CMOS VLSI Design," Addison-Wesley, 1985.

12 K.M. Ware et al., "A $200 \mathrm{MHz}$ CMOS Phase-Locked Loop with Dual Phase Detectors," IEEE Int'l Solid-State Ccts. Conf., pp. 192-193, 1989.

13 K. J. Negus et al., "Silicon Bipolar Mixed-Signal Farameterized-Cell Array for Wireless Applications to 4GHz,'IEEE Int'l Solid-State Ccts. Conf., pp. 230-231, 1992.

14 Simplified User Guide to the Cadence VLSI Design Software, Report ICI-023 RI, Rev: 1. Canadian Microelectronic Corporation, June 1, 1990. 

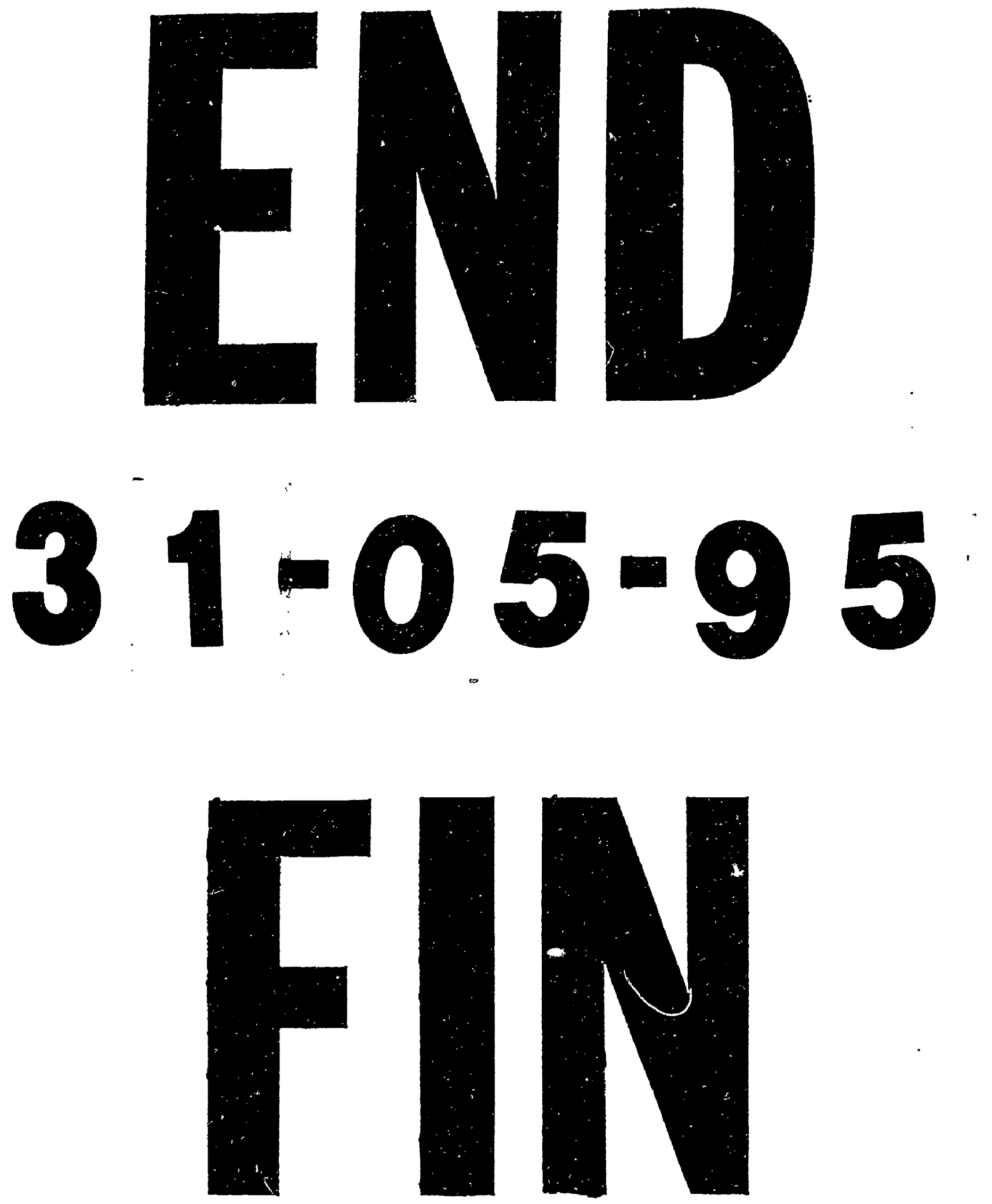